\title{
Automatic shear wave splitting measurements at Mt. Ruapehu volcano, New Zealand
}

by

Andreas Wessel

A thesis submitted to Victoria University of Wellington for the degree of Master of Science in Geophysics

Institute of Geophysics

School of Geography, Environment and Earth Sciences Victoria University of Wellington

Wellington, New Zealand

July 2010 


\section{Abstract}

This thesis presents an automatic shear wave splitting measurement tool and the results from its application to data recorded in the vicinity of Mt. Ruapehu volcano on the North Island of New Zealand. The best methodology and parameters for routine automatic monitoring are determined and approximately 10,000 events are processed. About $50 \%$ of all S-phases lead to measurements of acceptable quality. Results obtained with this technique are reproducible and objective, but more scattered than results from manual measurements.

The newly developed automatic measurement tool is used to measure shear wave splitting for previously analysed data and for new data recorded in 2003-2007. In contrast to previous studies at Mt. Ruapehu, we have a larger and continuous data set from numerous three-component seismic stations. No major temporal changes are found within the new data, but results vary for different station locations. 


\section{Acknowledgements}

This project would not have been possible without the help and support of numerous people and institutions. I want to thank the Earthquake Commission for the funding of the project. Without this funding I could not have done this work. GNS Science also contributed generously, with manpower and by providing all the data from the GeoNet stations.

I acknowledge the New Zealand GeoNet project and its sponsors EQC, GNS Science and FRST, for providing most of the data used in this study. While my predecessors had to plan and realise their own deployments of seismic stations, I could stay in a dry and warm place during the whole project and instantaneously start to work on the data processing.

I want to thank Martha Savage for the supervision of my work and for giving me the opportunity to do my masters thesis about this interesting topic in New Zealand. Martha Savage and Tony Hurst supervised this work and made countless valuable comments and suggestions that improved my research and the manuscript. Librarian Gillian Ruthven gave kind advice regarding the layout of this thesis. I would also like to thank Bronwyn O'Keefe, Anya Seward and Thomas Roehr for proofreading the manuscript.

Several programs that I used throughout the project were initially made by coworkers, many of them by Alexander Gerst. Maps and most of the diagrams were created with GMT (Generic Mapping Tools). Seismograms have been processed in the envi- 
ronment of the Seismic Analysis Code (SAC). The thesis was written with the freely available software $\mathrm{HT}_{\mathrm{E} X}$.

On a more personal matter, I appreciated the great community of people in the Waiteata University Hall. Getting to know students from all over the world has been a priceless experience. Thank you Katrine, Samantha, Chris, Thomas, Jianhui and Laine for being great flatmates during the year. 


\section{Contents}

Abstract I

Table of Contents IV

List of Figures . . . . . . . . . . . . . . . . . . . . VII

List of Tables ........................ . . X

\section{Chapters}

1. Introduction 1

1.1. Forecasting of volcanic eruptions . . . . . . . . . . . . 2

1.2. Volcanism in New Zealand and Mt. Ruapehu . . . . . . . . . . . . . . 3

1.2.1. Tectonics and geology of New Zealand . . . . . . . . . . . . 4

1.2.2. Volcanism in New Zealand . . . . . . . . . . . . . . 7

Central Volcanic Region . . . . . . . . . . . . . 8

Taupo Volcanic Zone . . . . . . . . . . . . . . . . . . . 8

1.2.3. Geology and historic activity of Mt. Ruapehu . . . . . . . . . . 10

1.2.4. Hazards at Mt. Ruapehu . . . . . . . . . . . . . . . . . . . . . 14

Current hazard $/$ risk . . . . . . . . . . . . . . . 15

Seismicity . . . . . . . . . . . . . . . 16

Monitoring . . . . . . . . . . . . . . . . 17

1.3. GeoNet network . . . . . . . . . . . . . . . . . . . . 19

2. Seismic anisotropy 21

2.1. Definition of seismic anisotropy . . . . . . . . . . . . . . . 21

2.1.1. Quantifying seismic anisotropy . . . . . . . . . . . 22

2.2. Causes of anisotropy . . . . . . . . . . . . . . . . . 23

2.2.1. Possible causes of mantle anisotropy . . . . . . . . . . . 25

2.2.2. Causes of crustal anisotropy . . . . . . . . . . . . . . 27

2.2.3. Microcracks . . . . . . . . . . . . . . . 28

2.3. Temporal changes in anisotropy (theory) . . . . . . . . . . . . . . . . 32

2.4. Stress: a connection to volcanic activity . . . . . . . . . . . . 34

2.4.1. Dike pressurising at Mt. Ruapehu . . . . . . . . . . . . . 35

3. Measuring anisotropy $\quad 37$

3.1. Wave equation for anisotropic media . . . . . . . . . . . 37 
3.2. Shear wave splitting . . . . . . . . . . . . . . . . . . 40

3.2.1. How to measure shear wave splitting . . . . . . . . . . . . . . 46

Spatial resolution . . . . . . . . . . . . . . 46

The shear wave window . . . . . . . . . . . . 47

Algorithms to measure shear wave splitting . . . . . . . . . 48

3.2.2. Complications . . . . . . . . . . . . . . . . . . . . . 48

$90^{\circ}$-flips . . . . . . . . . . . . . . . . . . . . 49

Cycle skipping . . . . . . . . . . . . . . . . 50

Null measurements . . . . . . . . . . . . . . . . . . 50

Geometrical complications . . . . . . . . . . . . 52

Distinguishing heterogeneous anisotropy . . . . . . . . . 53

3.2.3. Other methods . . . . . . . . . . . . . . 54

3.3. Automatic splitting measurements . . . . . . . . . . . . . . . 54

3.4. Observations of seismic anisotropy . . . . . . . . . . . . . 56

3.4.1. Crustal anisotropy and its applications . . . . . . . . . . 57

3.4.2. Temporal changes in seismic anisotropy (observations) . . . . 58

3.4.3. Anisotropy monitoring at active volcanoes . . . . . . . . . . . 61

3.4.4. Anisotropy in New Zealand and the regional stress field . . . . 63

3.4.5. Results from previous studies at Mt. Ruapehu . . . . . . . . . 64

Miller and Savage: 1994 and 1998 deployment . . . . . . . . . 64

Gerst and Savage: 2002 deployment . . . . . . . . . . . . 66

4. Method 69

4.1. Selection and retrieval of data . . . . . . . . . . . . . . 70

4.2. Preparation of data . . . . . . . . . . . . . . . . . . 71

4.2.1. Application of bandpass filters . . . . . . . . . . . . . 72

4.2.2. Signal to Noise Ratio (SNR) . . . . . . . . . . . . . . . . . 74

4.3. Shear wave splitting measurement . . . . . . . . . . . . . . . . . . . . . . . . . . . . 75

4.3.1. The Silver \& Chan algorithm . . . . . . . . . . . . . . 76

4.3.2. Measurement window . . . . . . . . . . . . . . 79

4.3.3. Cluster analysis . . . . . . . . . . . . . . . 81

4.3.4. Calculating the mean fast orientation . . . . . . . . . . . 83

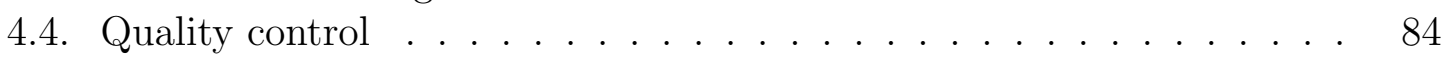

4.4.1. Cycle skipping . . . . . . . . . . . . . . . . . 85

4.4.2. Null measurements . . . . . . . . . . . . . . . . . 86

4.5. Illustrating the results . . . . . . . . . . . . . . . . . . . 88

4.6. Routine automatic measurements and monitoring . . . . . . . . . 90

5. Data used for shear wave splitting measurements 91

5.1. Data from previous studies . . . . . . . . . . . . . . . . 91

5.1.1. The 2002 CHARM deployment . . . . . . . . . . . . . . 91

5.2. GeoNet data . . . . . . . . . . . . . . . . . . 92

5.2.1. GeoNet data event details . . . . . . . . . . . . . . . . 92 
$\begin{array}{lr}\text { 6. Results } & \mathbf{9 7}\end{array}$

6.1. Development of an automatic measurement technique . . . . . . . . . 97

6.2. Shear wave splitting measurements . . . . . . . . . . . . . . 98

6.2.1. Reprocessing data from previous studies . . . . . . . . . . . 98

6.2.2. New results from data collected since 2002 . . . . . . . . . . . 104

$\begin{array}{ll}\text { 7. Discussion } & 127\end{array}$

7.1. The newly developed automatic method . . . . . . . . . . . . . 127

7.1.1. Comparison with other methods . . . . . . . . . . . . . 129

7.1.2. Caveats of this new method . . . . . . . . . . . . . . 131

7.2. Changes between 2002 and the new data set (2003-2007) . . . . . . . 132

7.3. Temporal changes within the new data set . . . . . . . . . . . . 132

7.4. Spatial changes within the new data set . . . . . . . . . . . 136

$\begin{array}{lr}\text { 8. Conclusions } & 139\end{array}$

8.1. Summary of the results . . . . . . . . . . . . . . . . 139

8.2. Prospects . . . . . . . . . . . . . . . . . . . 140

8.2.1. This method as a monitoring tool at Mt. Ruapehu . . . . . . . 140

8.2.2. This method at other localities . . . . . . . . . . . . . . 142

8.2.3. What can be improved? . . . . . . . . . . . . . . . 142

\section{Appendix}

A. User Manual

A.1. Basic knowledge of geophysical terms . . . . . . . . . . . . . . . . 143

A.2. Prerequisites . . . . . . . . . . . . . . . . . . 143

A.2.1. Customise paths and variables . . . . . . . . . . . . 144

A.2.2. Programming style and syntax . . . . . . . . . . . . . 144

A.3. Download and convert data . . . . . . . . . . . . . . . 145

Extract SAC files . . . . . . . . . . . . . . . . . . 146

A.3.1. Prepare data for splitting measurements . . . . . . . . . . . 147

A.4. Processing of data for splitting measurement . . . . . . . . . . . . 148

A.4.1. Find best filter . . . . . . . . . . . . . . . . . . . . . 149

A.4.2. Calculate signal-to-noise ratio . . . . . . . . . . . . . . 149

A.4.3. Create configuration file and measure shear wave splitting . . 151

Write results into logfile . . . . . . . . . . . . 152

A.4.4. Display results . . . . . . . . . . . . . . . . 154

A.5. Automatic measurement and monitoring tool . . . . . . . . . . . 155

A.6. 3rd-party software . . . . . . . . . . . . . . . 157

A.7. Cheatsheet . . . . . . . . . . . . . . . . . 158

$\begin{array}{ll}\text { B. Properties of the seismic data } & 161\end{array}$

$\begin{array}{lr}\text { C. Measurement results displayed on split maps } & 163\end{array}$

C.1. Yearly data sets, all stations . . . . . . . . . . . . . . . . 163 
C.2. Results of single stations . . . . . . . . . . . . . . . 166

C.3. Backazimuthal distribution of sources . . . . . . . . . . . . . . 172

\section{References and Index}

References . . . . . . . . . . . . . . . . . . . . 175

Index . . . . . . . . . . . . . . . . . . . . . . . . . 194 


\section{List of Figures}

1.1. Histogram: Volcanic incidents in the 20th century . . . . . . . . . . . 2

1.2. Maps: Volcanoes of New Zealand; Ruapehu . . . . . . . . . . . . . . 4

1.3. Map: Submarine shelves of Zealandia . . . . . . . . . . . . . 5

1.4. Map: Velocity field of the North Island . . . . . . . . . . . . . . . . . 6

1.5. Map: CVR and TVZ . . . . . . . . . . . . . . . . . 9 9

1.6. Photography of Mt. Ruapehu (in 2002) . . . . . . . . . . . . . . . . . 10

1.7. Satellite image of Mt. Ruapehu (in 2002) . . . . . . . . . . . . . . . . 11

1.8. Map: Ring plain surrounding Mt. Ruapehu . . . . . . . . . . . . . . . 12

1.9. Map: GeoNet stations on the North Island . . . . . . . . . . . . . . . 20

2.1. Sketch: Layer anisotropy . . . . . . . . . . . . . . . . . . . 24

2.2. Sketch: Lattice Preferred Orientation (LPO) . . . . . . . . . . . . 26

2.3. Sketch: Microcrack alignment . . . . . . . . . . . . . 29

2.4. Flowchart: Volcanic activity, stress and anisotropy . . . . . . . . . . . 35

2.5. Model: Stress changes caused by a magmatic dike . . . . . . . . . . . 36

3.1. Sketch: Effect of transverse isotropy on wave velocities . . . . . . . . 43

3.2. Sketch: Crack-induced shear wave splitting . . . . . . . . . . . . . 44

3.3. Sketch: Cycle skipping . . . . . . . . . . . . . . . . . 50

3.4. Sketch: Null measurements . . . . . . . . . . . . . . . . . . . . 51

3.5. Plot: Components of a null measurement . . . . . . . . . . . . 52

3.6. Plot: Changes in anisotropy at Mt. Etna (Italy) . . . . . . . . . . . . 62

3.7. Map: Stations of the 2002 CHARM deployment . . . . . . . . . . . . 67

3.8. Histograms: Results of CHARM project . . . . . . . . . . . . . 68

4.1. Flowchart: Processing steps for splitting measurements . . . . . . . . 70

4.2. Plot: Design of bandpass filter . . . . . . . . . . . . . . . . . . . . 72

4.3. Plots: Comparison of bandpass filters . . . . . . . . . . . . . . . 73

4.4. Plot: Amplitude spectrum of raw and filtered data . . . . . . . . . . 74

4.5. Sketch: Splitting parameters . . . . . . . . . . . . . . 75

4.6. Plot: Particle motion . . . . . . . . . . . . . . . . . 78

4.7. Plot: Error surface of grid search . . . . . . . . . . . . . . . . 79

4.8. Plot: Measurement windows in cluster analysis code . . . . . . . . . . 80

4.9. Plot: Cluster analysis . . . . . . . . . . . . . . . 81

4.10. Plots: Null measurements . . . . . . . . . . . . . . . . . . 86

4.11. Rose diagram (example) . . . . . . . . . . . . . . . . . . . . . . 88

4.12. Shear wave splitting analysis plot . . . . . . . . . . . . . 89 
5.1. Map: GeoNet stations at Mt. Ruapehu . . . . . . . . . . . . . . . . 93

5.2. Plot: Run times of GeoNet stations . . . . . . . . . . . . . . . 95

5.3. Maps: Selected event data. . . . . . . . . . . . . . . . . . . 96

6.1. Plots: Comparison of manual and automatic method. . . . . . . . . . 99

6.2. Histograms: Results from 1994 and 1998 data . . . . . . . . . . . . . 100

6.3. Maps: Results from 2002 data (manual) . . . . . . . . . . . . . . . . 102

6.4. Maps: Results from 2002 data . . . . . . . . . . . . . . . . . . . . 102

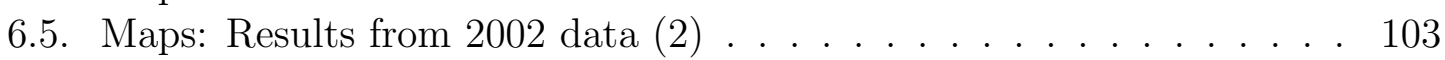

6.6. Maps: Results from 2004 data . . . . . . . . . . . . . . . . . . . . 109

6.7. Maps: Results from 2005 data . . . . . . . . . . . . . . . . . . . . 110

6.8. Maps: Results from 2006 data . . . . . . . . . . . . . . . . . . 111

6.9. Maps: Results from 2007 data . . . . . . . . . . . . . . . . . . 112

6.10. Maps: Results from 2004-2006 data . . . . . . . . . . . . . . . . . 113

6.11. Plot: Example of moving-window average . . . . . . . . . . . . . . 114

6.12. Plot: Results vs time, station TRVZ . . . . . . . . . . . . . . 115

6.13. Plot: Results vs time, station FWVZ . . . . . . . . . . . . . 116

6.14. Plot: Results vs time, station WNVZ . . . . . . . . . . . . . 117

6.15. Plot: Results vs time, station TUVZ . . . . . . . . . . . . . 118

6.16. Plot: Results vs time, station WPVZ . . . . . . . . . . . . . . . 119

6.17. Plot: Results vs time, station NGZ . . . . . . . . . . . . . . 120

6.18. Plot: Results vs time, station MTVZ . . . . . . . . . . . . . . 121

6.19. Plot: Results vs time, station OTVZ . . . . . . . . . . . . . . 122

6.20. Plot: Results vs time, station WTVZ . . . . . . . . . . . . . . . 123

6.21. Plot: Results vs time, station PKVZ . . . . . . . . . . . . . . . . 124

6.22. Plot: Results vs time, station MOVZ . . . . . . . . . . . . . . . 125

6.23. Plot: Results vs time, station TWVZ . . . . . . . . . . . . . . 126

7.1. Maps: Results from station MOVZ (shallow) . . . . . . . . . . . . 135

7.2. Maps: Results from 2007 data, selected stations . . . . . . . . . . . 136

7.3. Maps: Histograms of event backazimuths. . . . . . . . . . . . . . . . . 137

A.1. Plots: Comparison of bandpass filters . . . . . . . . . . . . . . . 150

A.2. Plot: Measurement window times . . . . . . . . . . . . . . . 151

A.3. Plots: Splitting analysis plot and rose diagrams . . . . . . . . . 156

B.1. Map: Selected events within $150 \mathrm{~km}$. . . . . . . . . . . . . 161

B.2. Plot: 3D projection of seismicity . . . . . . . . . . . . . . 162

C.1. Maps: Results from 2004 data . . . . . . . . . . . . . . . . . . 163

C.2. Maps: Results from 2005 data . . . . . . . . . . . . . . . . . . . . 164

C.3. Maps: Results from 2006 data . . . . . . . . . . . . . . . . . . . . 164

C.4. Maps: Results from 2007 data . . . . . . . . . . . . . . . . . 165

C.5. Maps: Results from 2004-2007 data . . . . . . . . . . . . . . . . . 165

C.6. Maps: Results from station TRVZ . . . . . . . . . . . . . . . . 166

C.7. Maps: Results from station FWVZ . . . . . . . . . . . . . . 166 
C.8. Maps: Results from station WNVZ . . . . . . . . . . . . . . 167

C.9. Maps: Results from station TUVZ . . . . . . . . . . . . . . . . 167

C.10.Maps: Results from station WPVZ . . . . . . . . . . . . . 168

C.11.Maps: Results from station NGZ . . . . . . . . . . . . . . 168

C.12.Maps: Results from station MTVZ . . . . . . . . . . . . . . 169

C.13.Maps: Results from station OTVZ . . . . . . . . . . . . . . . 169

C.14.Maps: Results from station WTVZ . . . . . . . . . . . . . . 170

C.15.Maps: Results from station PKVZ . . . . . . . . . . . . . . . 170

C.16.Maps: Results from station MOVZ . . . . . . . . . . . . . . 171

C.17.Maps: Results from station TWVZ . . . . . . . . . . . . . . 171

C.18.Maps: Histograms of event backazimuths. . . . . . . . . . . . . . . . . 172

C.19.Maps: Histograms of event backazimuths. . . . . . . . . . . . . . . . . 173

C.20.Maps: Histograms of event backazimuths. . . . . . . . . . . . . . . 173

C.21.Maps: Histograms of event backazimuths. . . . . . . . . . . . . . . 174 


\section{List of Tables}

2.1. Anisotropy of mantle minerals . . . . . . . . . . . . . . 25

2.2. Anisotropy of crustal minerals . . . . . . . . . . . . . . . . 27

3.1. Results from Miller and Savage (2001) . . . . . . . . . . . . . . 65

3.2. Results from Gerst and Savage (2004) . . . . . . . . . . . . 66

4.1. Spatial event selection criteria . . . . . . . . . . . . . . 71

5.1. Stations of the GeoNet network . . . . . . . . . . . . . . . 94

6.1. Results from the 1994 and 1998 deployments . . . . . . . . . . . . . . 100

6.2. Results from the 2002 deployment . . . . . . . . . . . . . . . . . . 101

6.3. Yearly results of individual stations . . . . . . . . . . . . . . . . . . 104

7.1. Statistics of the automatic processing . . . . . . . . . . 130

A.1. Fields of results logfile . . . . . . . . . . . . . . . 153 


\section{Introduction}

Monitoring of active volcanoes and efforts to forecast volcanic eruptions remain a challenge for earth scientists. It is important to monitor active volcanoes and to foster improvements and new techniques. Mt. Ruapehu is an active volcano on the North Island of New Zealand. It had hazardous activity in the past, and temporal changes in seismic anisotropy have been said to coincide with volcanic activity (Miller and Savage, 2001; Gerst and Savage, 2004). Seismic anisotropy can be measured with a seismological technique called shear wave splitting.

If done manually, those measurements are time-consuming and laborious. To routinely monitor changes in seismic anisotropy and to achieve results quickly, but still reliably, an automation of the process is desirable. Automation will hopefully contribute to an eruption forecasting tool from routine monitoring of seismic anisotropy at Mt. Ruapehu, and possibly other volcanoes. An automatic shear wave splitting measurement technique has the potential to be used with other data sets that are recorded with three-component stations.

The main goal of this study is to automate the anisotropy analysis. Another goal of this study is to investigate anisotropy changes in new data since 2002. There are now several permanent three-component seismic stations in the vicinity of Mt. Ruapehu that allow continuous anisotropy monitoring. 
Thus, the two key points of this study are:

- Development of an automatic measurement tool

- Measuring anisotropy with new data (2002-2007) and interpreting the results

\subsection{Forecasting of volcanic eruptions}

For the 20th century, Witham (2005) catalogued 491 volcanic events world-wide, out of which 260 events caused fatalities. All reported events that caused "incidents" are included, which in this context is defined as having impact on human life, such as death, injuries, homelessness, or being affected or evacuated (Witham, p. 194). In this time period, about 5.6 million people were affected or evacuated, more than 90,000 were killed and almost 300,000 were left homeless.

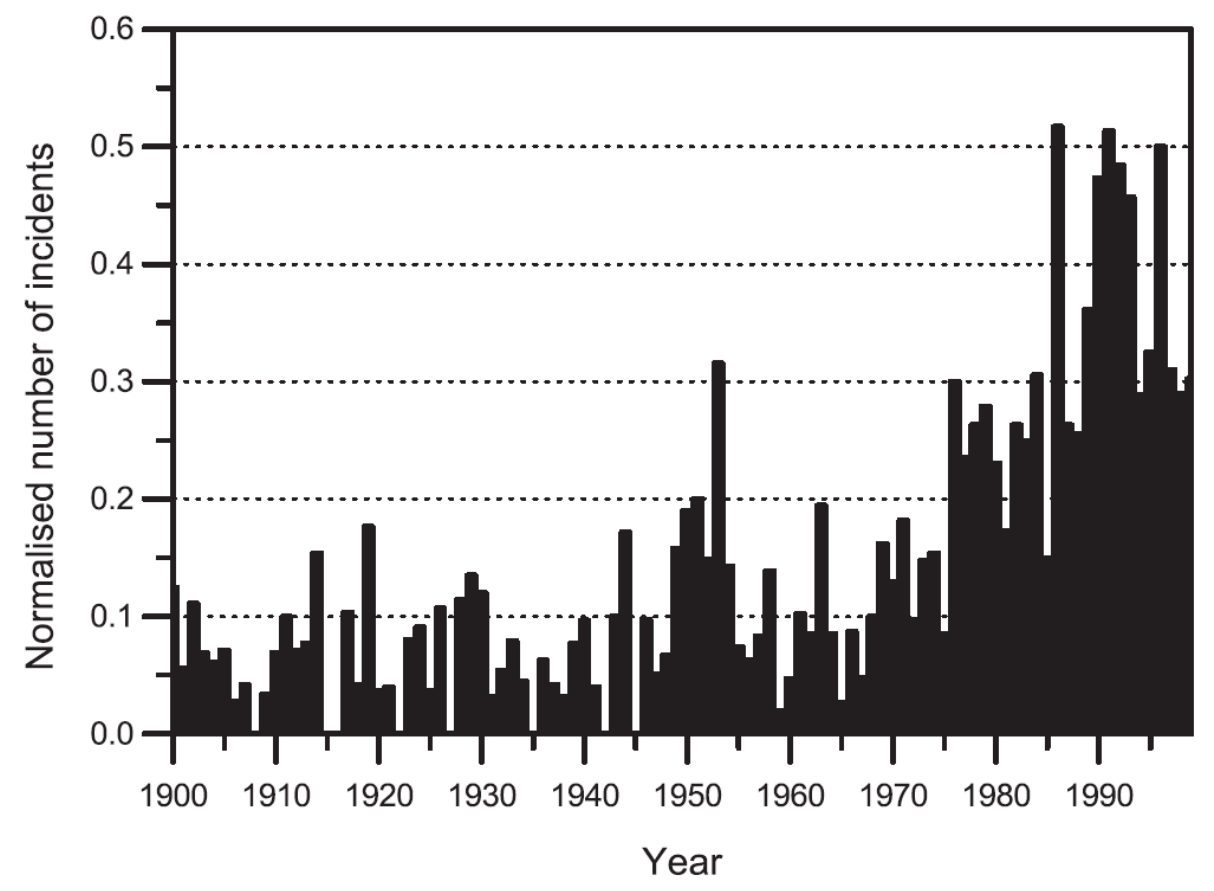

Figure 1.1.: Events that were classified as volcanic incidents in the 20th century (per year, normalised by the total number of eruptions). The percentage of incidents has increased in the last decades. (Source: Witham, 2005) 
In the last decades, the number of incidents per eruption has increased (Figure 1.1). That means for a fixed number of eruptions (say 100) a higher portion were classified as "incidents". It is not entirely clear if this increase is due to better reporting of volcanic events and their impact, more extensive use of evacuations, actual changes in volcanic activity, or a higher vulnerability due to increased population adjacent to active volcanoes (Witham, 2005). But whatever combination of those hypotheses proves true, impact of volcanic activity on human life is either increasing, or was underestimated in the past. In total, $12 \%$ of the world's population live within a $100 \mathrm{~km}$ radius of a volcano that has been active in the last 10,000 years (state of 1990; Small and Naumann, 2001).

New Zealand has several active volcanoes (Figure 1.2a). While there are efforts for short-term warning, e.g. a seismicity-based eruption detection system, mid- and long-term trends are an important indicator for the status of a volcano (e.g. Scarpa and Tilling, 1996). Monitoring of the crater lake temperature and chemistry provides information about the heat and gas flux (see Hurst et al., 2004, for an overview of volcano monitoring in New Zealand).

Anisotropy monitoring has the capability to provide information about the state of stress underneath Mt. Ruapehu and can therefore contribute to any effort in volcano monitoring. By monitoring temporal changes in anisotropy beneath Mt. Ruapehu volcano (Figure 1.2b) it is hoped to facilitate another eruption forecasting tool. The connection between volcanic activity, stress changes and anisotropy is explained in Section 2.4, p. 34 .

\subsection{Volcanism in New Zealand and Mt. Ruapehu}

The geology of Mt. Ruapehu volcano and the hazards occurring in the course of volcanism at Tongariro National Park are described in this section. The tectonics 


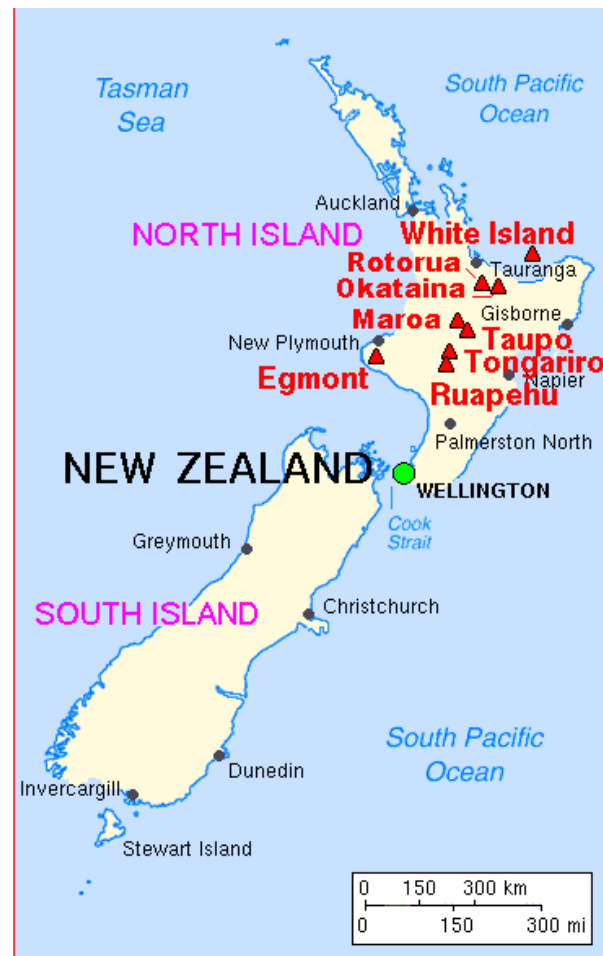

(a) Major volcanoes of New Zealand

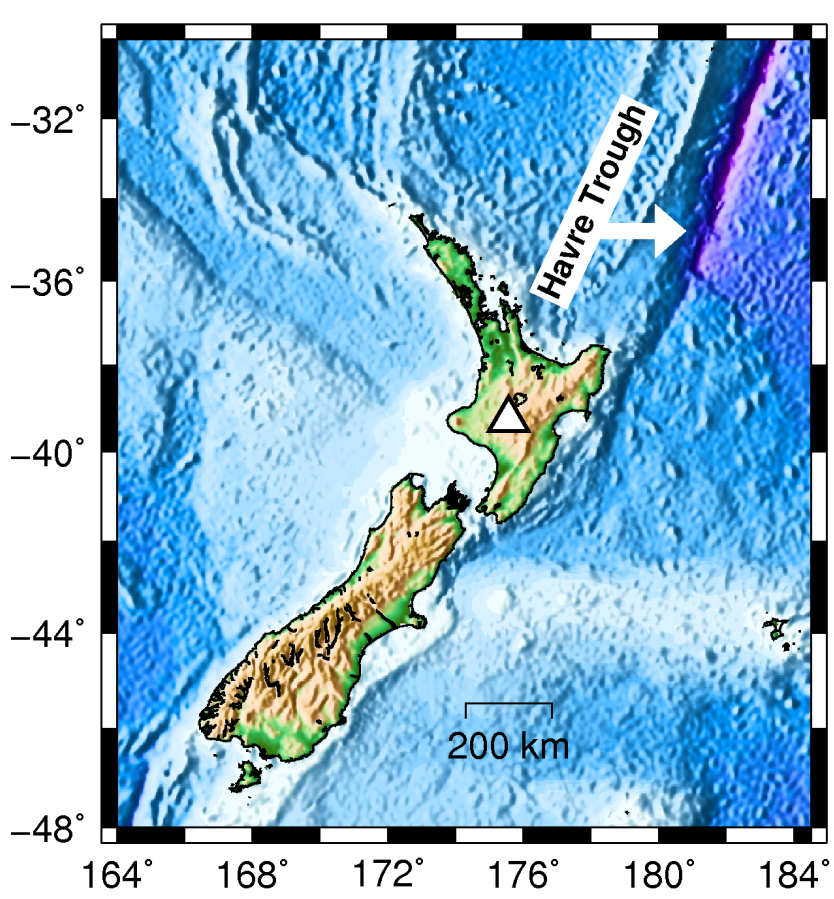

(b) Location of Mt. Ruapehu in New Zealand

Figure 1.2.:

a) Major volcanoes of New Zealand (Triangles mark active volcanoes, circles mark cities). Volcanic activity is concentrated on the North Island. (Source: U.S. Geological Survey, http://vulcan.wr. usgs. gov/Imgs/ Gif/NewZealand/Maps/map_new_zealand_volcanoes.gif, June 21, 200\%. Used with permission.)

b) Location of Mt.Ruapehu (triangle) on the North Island of New Zealand. The Havre Trough is visible NNE of the North Island.

and geology of New Zealand are briefly summed up. After describing the Taupo Volcanic Zone, the geology of Mt. Ruapehu, as well as hazards and seismicity are depicted.

\subsubsection{Tectonics and geology of New Zealand}

The New Zealand continent is sometimes referred to as Zealandia (Figure 1.3). Only a small part of Zealandia's area is above sea level and forms the New Zealand landmass, while approx. $90 \%$ of the area is submerged in ocean water, forming large 


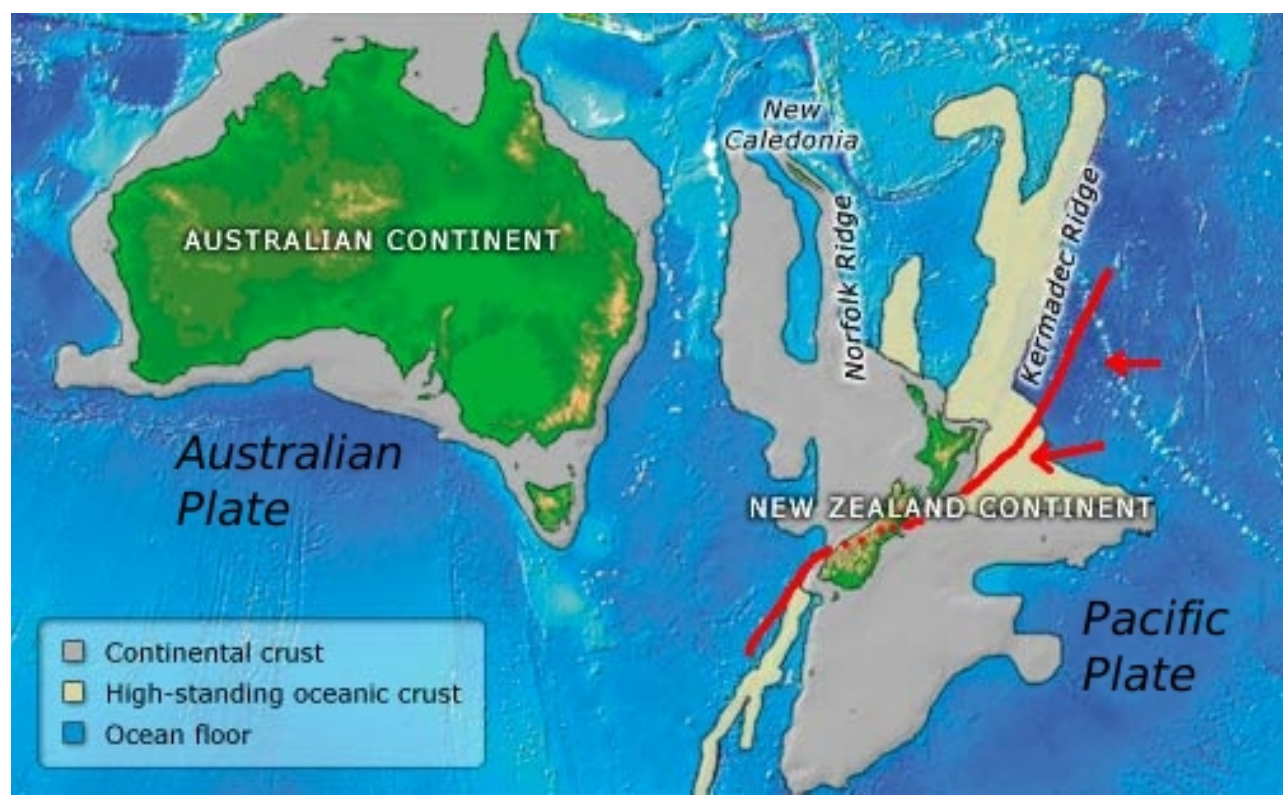

Figure 1.3.: Submarine shelves of Zealandia. A large proportion of the continent Zealandia is submerged in ocean water. (Source: NIWA, http://www. niwa. co.nz, used with permission.) The plate boundary between Australian Plate and Pacific Plate is drawn in red. Arrows indicate the relative motion between the plates at the Hikurangi Margin (see text for details).

continental shelves (Mortimer, 2004). The total area of the continent is more than $5,000,000 \mathrm{~km}^{2}$ (Wood and Barker, 2005).

The landmass of New Zealand split from Antarctica in the Cretaceous (Mortimer, 2004; Mortimer et al., 2006). The ages of volcanic rocks on the North and the South Island were used to infer that the breakup caused lithosphere detachment and upwelling of the asthenosphere due to gravitational instabilities in the continental lithosphere of Zealandia (Hoernle et al., 2006; see also Stern et al., 2006, and references therein). This led to Cenozoic intraplate volcanism.

At present New Zealand is situated at the boundary of two tectonic plates. The oceanic crust of the westward-moving Pacific plate is being replaced by new oceanic crust formed at the East Pacific Rise by being subducted underneath the continental crust of the North Island (Australian plate). This oblique subduction process yields the Hikurangi Margin. Underneath the southern South Island the Australian plate is 


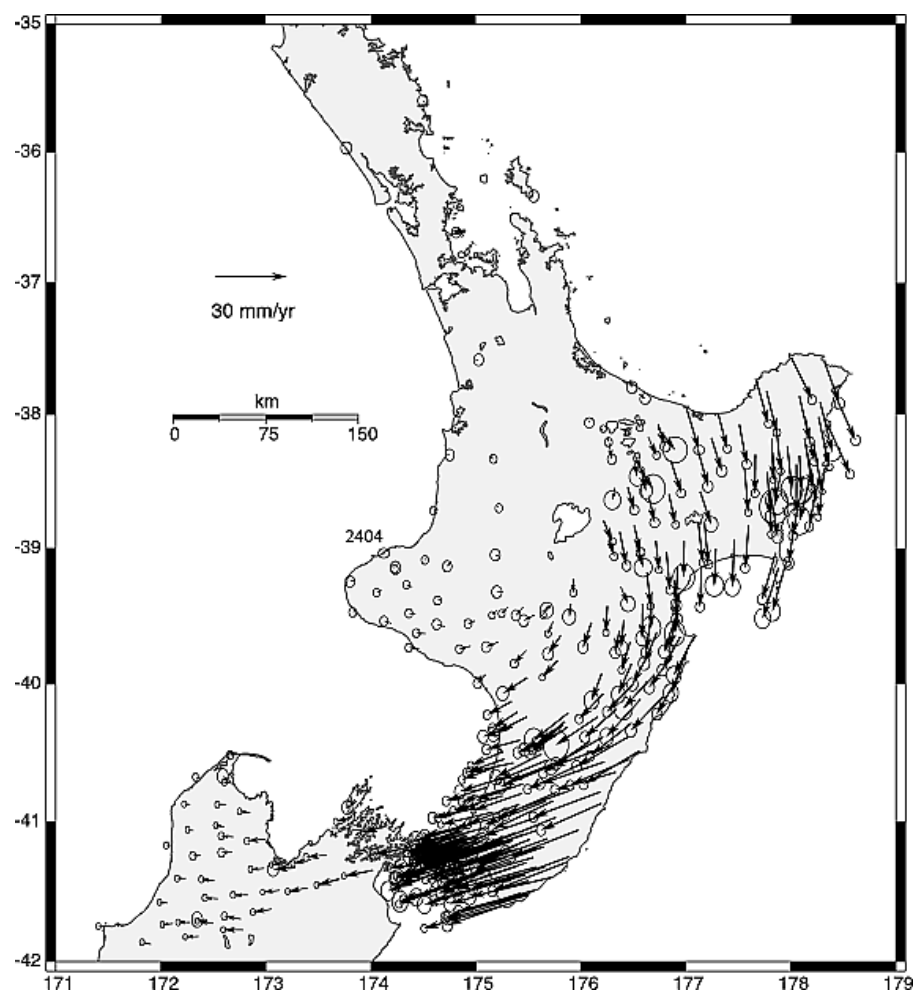

Figure 1.4.: Velocity field of the North Island (relative to the Australian Plate), derived from regional GPS surveys. These observations show that the North Island is rotating clockwise (Wallace et al., 2004). The same authors predict an extension rate of the TVZ of $5-15 \mathrm{~mm} / \mathrm{a}$, with higher rates in the northern part and the lowest rates at the southern end. (Source: Wallace et al., 2004)

subducted eastwards at the Fjordland Margin and the Puysegur Trench (Christoffel and van der Linden, 1972). Between the two subduction zones the intersection of the tectonic plates is visible as the Alpine Fault, a dextral transform fault dissecting the South Island (Sutherland, 1999, and references therein). The Hikurangi margin has been characterised as a zone where plate coupling and large earthquakes take place (e.g. Reyners, 1998).

Regional GPS (Global Positioning System) surveys (Wallace et al., 2004) show that the eastern North Island is rotating clockwise (relative to the Australian plate, see Figure 1.4). Several geophysical and geological observations support this estimate (Stern et al., 2006). At the Hikurangi Margin off the east coast of the North Island, the 
Pacific plate is subducted at a rate of $\sim 60 \mathrm{~mm} / \mathrm{a}^{*}$ at $35^{\circ} \mathrm{S}$ and at $\sim 50 \mathrm{~mm} / \mathrm{a}$ at $42^{\circ} \mathrm{S}$, while the subduction rate is slightly more than $50 \mathrm{~mm} / \mathrm{a}$ at Mt. Ruapehu's latitude (Gripp and Gordon, 2002). Tomographic inversions of seismic velocities indicate that the subducted Pacific plate is located at a depth of about $100 \mathrm{~km}$ underneath Mt. Ruapehu (Reyners et al., 2006).

\subsubsection{Volcanism in New Zealand}

New Zealand is part of the Pacific Ring of Fire, an area known for earthquakes and volcanic activity. The activity of New Zealand's volcanoes remains a focus of the public and media (e.g. Dominion Post, August 5, 2006; Taranaki 'to erupt within 50 years'). With the presence of a subduction zone along the North Island the question arises why does volcanism occur in the northern and central North Island but not on the southern part of the North Island? (see Figure 1.2a, p.4).

Interseismic plate coupling is considered to be a possible explanation (Mooney, 1970; Walcott, 1979; Eberhart-Phillips et al., 2005). The cessation of volcanism in the southern part of the North Island is proposed to be caused by the influence of the dry Haast schist belt, which prevents fluids from being distributed into overlying mantle rocks in the volcano-free southern part. The strong coupling of lithosphere and subducting plate in the southern part of the Hikurangi Margin led to the development of a back-arc basin, because the lithosphere of the subducted Pacific plate is bent downwards (Eberhart-Phillips et al., 2005). It should be noted here that Mt. Ruapehu may be a special case of a volcano located in an area of extension changing to compression (Wallace et al., 2004; Villamor and Berryman, 2006a).

\footnotetext{
*Velocities are calculated from the HS3-Nuvel 1A model, after Gripp and Gordon (2002), with the UNAVCO plate motion calculator, http://sps . unavco.org/crustal_motion/dxdt/ nnrcalc/, June 21, 2007.
} 


\section{Central Volcanic Region}

The Central Volcanic Region (CVR; Figure 1.5) is a wedge-shaped basin in the North Island, a rift zone, defined geophysically by its negative gravity anomaly. It is the continuation of an oceanic back-arc basin, the Havre Trough. Thus, the CVR hosts a rare example of back-arc basin formation in continental crust (Stern, 1985). The subduction process induces back-arc spreading, causing a gravity anomaly by extending the crust in this region. Stern (1985) explained residual anomalies of -55 to $-60 \mathrm{mGal}$ with the presence of low-density volcanic rocks and possibly bodies of molten magma at depth. For a detailed description of the CVR see Stern (1985, 1987) and Stratford and Stern (2006). Faults in the CVR are dense, predominantly normal faults (due to the extensional regime) and strike generally NE (Berryman and Beanland, 1991; Villamor and Berryman, 2006b).

\section{Taupo Volcanic Zone}

The Taupo Volcanic Zone (TVZ) is located in the eastern part of the CVR. It is named after Taupo, its largest volcano, which erupted violently in $180 \mathrm{AD}$ and ejected about $100 \mathrm{~km}^{3}$ of volcanic material (Smith and Houghton, 1995, and references therein). At the time of the eruption New Zealand was uninhabited by humans. The resulting large caldera is known as Lake Taupo. The eastern boundary of the TVZ is represented by a line of active younger arc-volcanoes - with Mt. Ruapehu at the southern tip-, while a back-arc basin is located in the center. The TVZ is characterised by active volcanism (Wilson et al., 1995), remarkably high heat flow $\left(700 \mathrm{~mW} / \mathrm{m}^{2}\right.$ in the central $6000 \mathrm{~km}^{2}$; Bibby et al., 1995), thinned crust $(\sim 15 \mathrm{~km})$ and low seismic velocities (Smith et al., 1989; Reyners et al., 2006) and has had "widespread" ignimbrite eruptions since 0.6 Ma ago (Cole, 1990). Erupted magmas in the TVZ are mainly rhyolitic. 


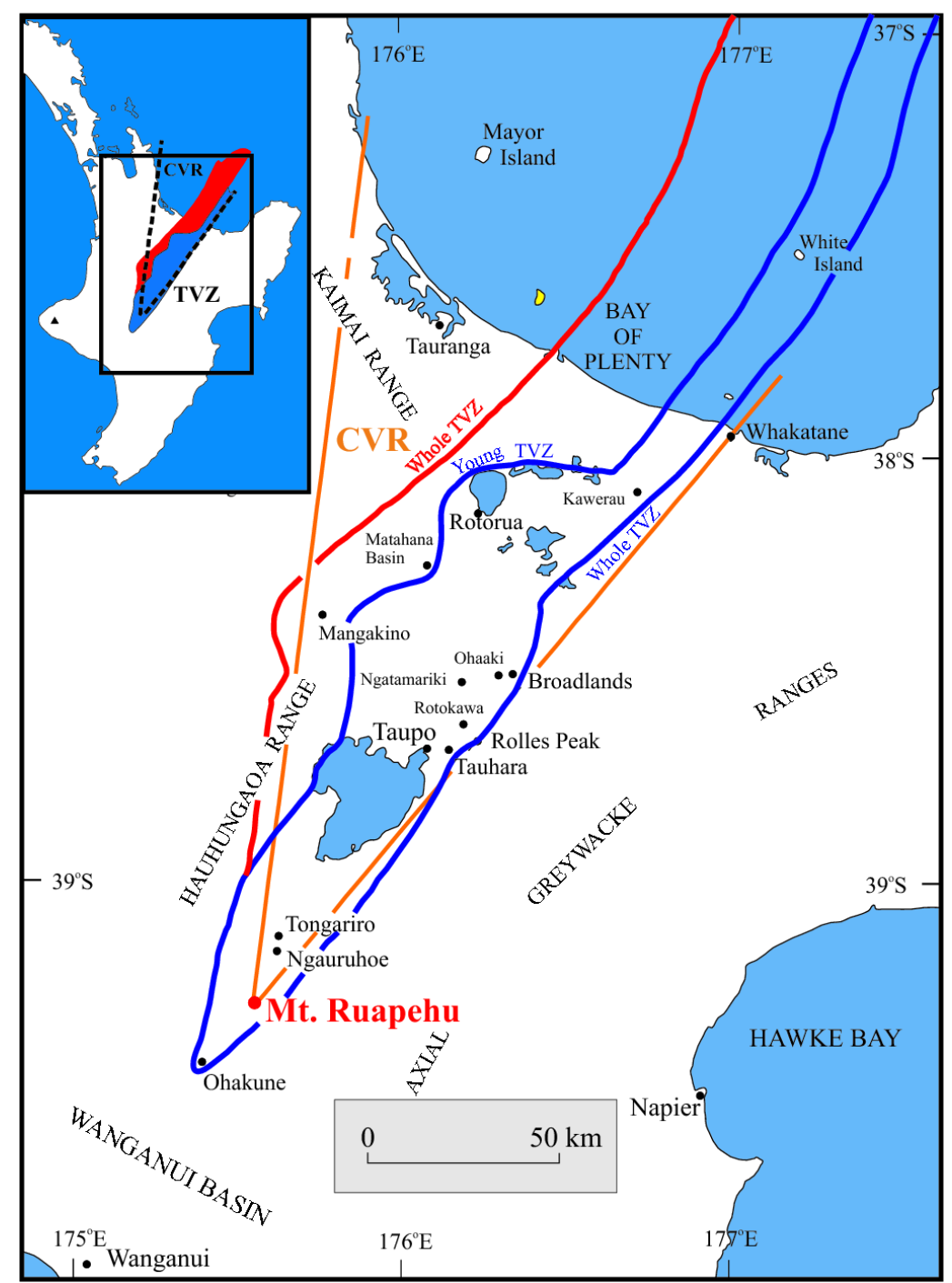

Figure 1.5.: Map of the CVR and the TVZ on the North Island of New Zealand. The young TVZ (blue) is a south-eastwards migrating front of young andesitic volcanoes. See text for details. Source: Gerst (2003), adapted from Wilson et al. (1995).

Volcanic activity of the TVZ started $\sim 2$ Ma ago*(Wilson et al., 1995), while backarc spreading started to form the CVR 4 Ma ago (Stern, 1987). The GPS velocity distribution is consistent with the current occurrence of rifting in the TVZ (Wallace et al., 2004). There is still andesitic volcanism (e.g. at Mt. Ruapehu and Tongariro at the southern end of the TVZ), but in the central part a change from andesitic

*Cole (1990) stated that the TVZ "has developed since 1 Ma" which presumably means the beginning of rifting that Wilson et al. (1995) dated 0.9 Ma. Ignimbrite deposits with a maximum age of 1.68 Ma have been reported by Houghton et al. (1995). 


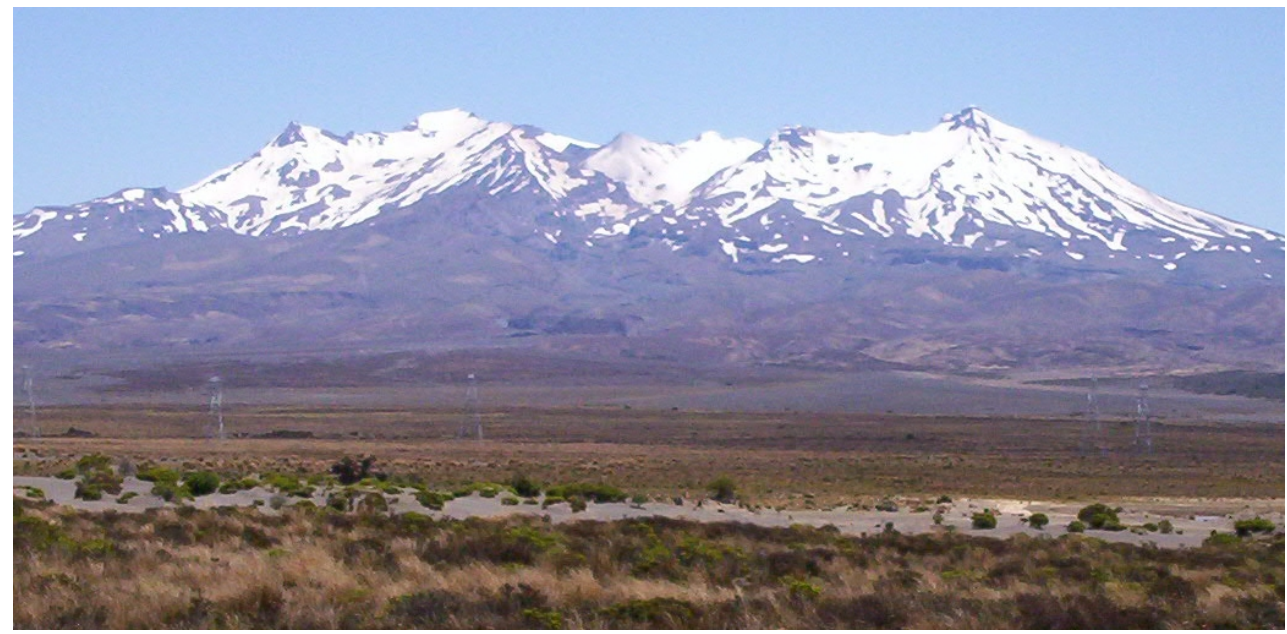

Figure 1.6.: Mt. Ruapehu in 2002 (Photo: Matt Angland)

to rhyolitic compositions took place over a few $100 \mathrm{ka}$, until rhyolitic magmatism dominated since approximately 100 ka ago (Price et al., 2005). The "young TVZ" (0.34 Ma to present) erupted $3000 \mathrm{~km}^{3}$ of rhyolites (Wilson et al., 1995). Southeastward migration of volcanic activity with time (Stern, 1987) defined the eastern boundary of TVZ and CVR (Figure 1.5). The extension rate (derived from GPS surveys; Wallace et al., 2004) is higher in the northern TVZ (15 mm/a) than at the southern end $(<5 \mathrm{~mm} / \mathrm{a})$. Widespread faulting at the southern end of the TVZ started less than $400 \mathrm{ka}$ ago, which supports the model of propagation of the TVZ to the south with time (Hatherton, 1970; Villamor and Berryman, 2006b).

\subsubsection{Geology and historic activity of Mt. Ruapehu}

Towering up to $2797 \mathrm{~m}$ above sea-level and hosting several glaciers, Mt. Ruapehu is the highest mountain in the North Island and is located at the southern end of the TVZ (Figures 1.5, 1.6 and 1.7). It is an active andesitic stratovolcano whose vent is filled with a crater lake. Ruapehu has erupted repeatedly through the same vent, so that it is classified as a polygenetic volcano (Nakamura, 1977; Wilson et al., 1995).

The four major formations at Mt. Ruapehu, Te Herenga (250-180 ka), Waihianoa 


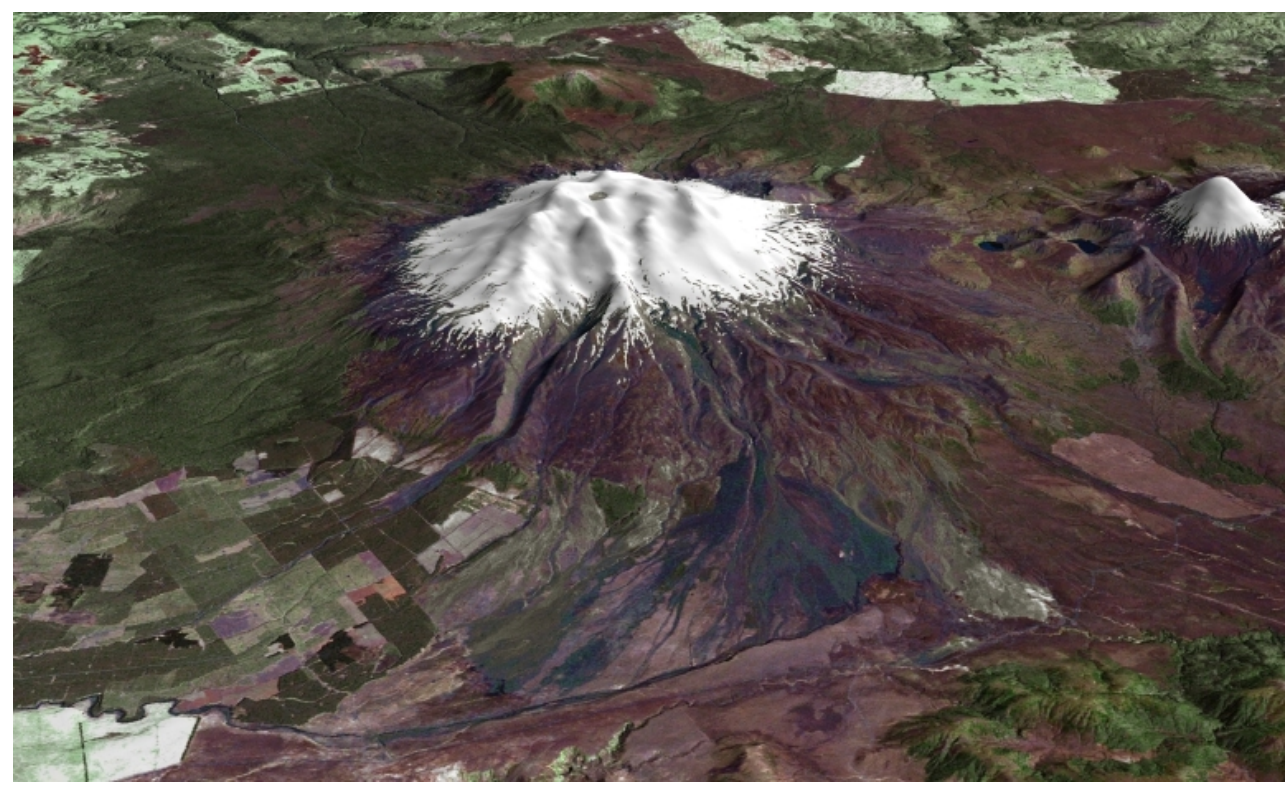

Figure 1.7.: Composite satellite image (landsat) of Mt. Ruapehu, as of 2002. The point of view is E-SE of Ruapehu. Agricultural land is visible behind the volcano. Crater Lake and the snow cap of the summit are visible, too. The cone on the right is Mt. Ngauruhoe; the interval between Mt. Ruapehu (Crater Lake) and Ngauruhoe is approximately $15 \mathrm{~km}$. (Source: NASA, http:// earthobservatory. nasa. gov/Newsroom/NewImages/ Images/landsat_mt_ruapehu_23oct02_lrg. jpg, June 22, 2007)

(160-115 ka), Mangawhero (55-45 and 30-15 ka) and Whakapapa (<15 ka) have been dated by Gamble et al. (2003). Evidence of former activity through other vents than the current one is exposed on Pinnacle Ridge, NW of Mt. Ruapehu, where fossil feeder systems (dikes) cut through the cone-building rocks (Gamble et al., 2003).

Underneath the cone of Ruapehu, Tertiary marine sediments are presumed to overlay a Mesozoic greywacke basement (Houghton et al., 1987; Rowlands et al., 2005). With seismic tomography inversion, Rowlands et al. (2005) found a low-velocity zone beneath Mt. Ruapehu. Possible explanations are: bending of the crust ("downwarping") due to the weight of the Ruapehu cone, sequences of low-velocity tephras, or, considered less likely, hydrothermal alteration or leftover partial melt from previous eruptions. 
Compared to the thin crust underneath the TVZ, the crust is thickened in the vicinity of Mt. Ruapehu (thickness of the crust is $35 \mathrm{~km}$ northeast and $40 \mathrm{~km}$ southwest of Ruapehu; Reyners et al., 2006).

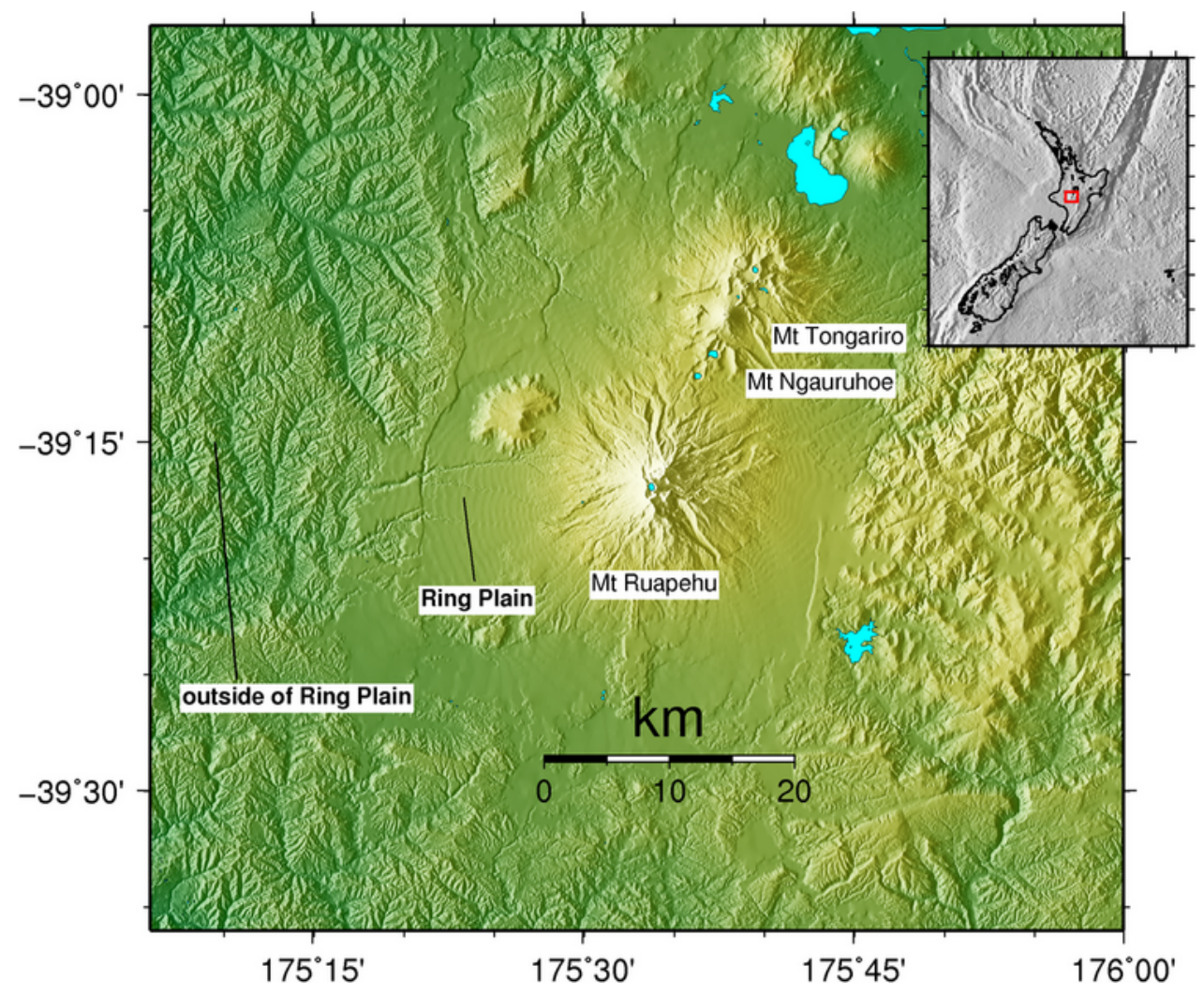

Figure 1.8.: Ring plain surrounding Mt. Ruapehu, visible in this graphical representation of a digital elevation model. Note the difference in topographic roughness compared to areas outside the ring plain. Volcanic deposits also cover the area around Mt. Ngauruhoe and Mt. Tongariro.

The area around Mt. Ruapehu is covered by numerous volcanic deposits (Figure 1.8). The Onetapu Formation in the southern ring plain contains a massive breccia unit, which indicates that debris avalanches reached further than $40 \mathrm{~km}$ from the summit (Lecointre et al., 2004; Thouret et al., 2006). The ring plain deposits, mostly avalanche debris and lahar deposits, have a low seismic velocity $\left(\mathrm{v}_{p}<4 \mathrm{~km} / \mathrm{s}\right.$; Rowlands et al., 2005).

Gamble et al. (2003) estimate $340 \mathrm{ka}$ as a maximum age for volcanic activity at Ruapehu and a cone volume of $\sim 110 \mathrm{~km}^{3}$ (a total volume of $300 \mathrm{~km}^{3}$ of volcanic 
material if deposits and tephras of the ring plain are included). These authors also estimate the average magma flux at Mt. Ruapehu as $0.6-1.2 \mathrm{~km}^{3} / \mathrm{ka}$. But activity is unsteady and these numbers have to be interpreted with caution, as the flux can be multiple times higher during active phases and flux averages also vary between different formations.

Mt. Ruapehu erupts low-K andesites with lower crustal xenoliths (granulites and meta-quarzites; Price et al., 2005). The broad compositional range of Mt. Ruapehu andesites is explained by magma modification in the crust. While magma is moving through crustal dikes and sills, melts are assimilated and crystals grow, separate and fractionate - and therefore the composition of the magma can be changed (Price et al., 2005). This is consistent with the postulated existence of small magma pockets, that possibly caused the compositional diversity of the 1995/96 eruptions* (Nakagawa et al., 1999).

Geochronological methods led to the discovery of past eruptive pulses that were dated 200, 134, 45, 22 and $<15$ ka ago (Gamble et al., 2003). The latest eruptions in the 20th century took place in 1945, 1966, 1969, 1971, 1975, 1977, 1995, 1996 (Nakagawa et al., 1999), with eruptions whose ejecta reportedly reached further than $100 \mathrm{~km}$ in 1945, 1975 and 1995/96 (Houghton et al., 1987; Johnston et al., 2000).

The most recent large eruptions (1995/96) produced andesitic scoria and bombs and were phreatomagmatic and strombolian. One eruption plume reached a height of $20 \mathrm{~km}$ in a June 1996 event, while its ash deposits reached as far as $200 \mathrm{~km}$ from the summit and bombs covered a distance of up to $1.5 \mathrm{~km}$ (Nakagawa et al., 1999; Sherburn et al., 1999). The eruption sequences of 1995 and 1996 occurred with only minor and inconsistent precursory phenomena (Bryan and Sherburn, 1999). Volcanic and seismic activity during the 1995/96 eruptions was described by Bryan and Sherburn (1999). The first eruption of the 1995/96 eruptive phase took place

\footnotetext{
*The 1995 andesites were mixing products of two magmas (Nakagawa et al., 1999).
} 
on 17 September and was preceded by two minor eruptions accompanied by volcanic earthquakes on 28 June and 2 July. Activity continued until the end of October 1995 and commenced again at 16 June 1996 before it ceased at the end of July in the same year. Minor hydrothermal eruptions were reported 4 October 2006 (Ruapehu Alert Bulletin 04, 2006) and 29 September 2007 (Ruapehu Alert Bulletin 03, 2007).

\subsubsection{Hazards at Mt. Ruapehu}

Mt. Ruapehu lies some $230 \mathrm{~km}$ N-NE of Wellington, $280 \mathrm{~km}$ S-SE of Auckland, and just $75 \mathrm{~km}$ SW of Taupo. Several small towns are located closer to the summit. The town of Waiouru is approximately $24 \mathrm{~km}$ away from the summit, Ohakune is only about $20 \mathrm{~km}$ away. There are three ski fields on the flanks of the volcano. Houghton et al. (1987) described three hazard zones at Mt. Ruapehu that occur with phreatomagmatic eruptions. In the inner zone, which surrounds the active vent, ballistics pose the highest risk. In the outer zone fall deposits like volcanic ash are predominant. The third zone is defined by high lahar risk and follows topographical features such as river valleys. Volcanic activity can have far-reaching effects, as was seen after Ruapehu's 1996 eruptions, when ash falls were reported in Auckland, Hamilton and Taupo (Johnston et al., 2000).

A tephra dam was formed by deposits from the 1945 eruption and later dammed up water atop the crater. This tephra dam broke in 1953, which caused a lahar and the Tangiwai Railway disaster (Houghton et al., 1987). The lahar damaged the Tangiwai railway bridge, which caused a passenger train to founder into the mudflow and took the lives of 151 people. The combination of crater lake, snow-cover, glaciers and volcanic activity is an important factor to determine Ruapehu's hazards, as lahars and mudflows can feed on crater lake water or melted snow and ice. In the course of the 1995/96 eruptions, lahars were not solely a direct consequence of eruptive activity, but were also triggered by rainfall. One lahar passed through a ski field that had 
been cleared only an hour earlier. Approximately 100 people were evacuated from ski-field huts in the course of the eruptions.

The cost of the 1995/96 eruptions was estimated higher than 130 million NZ $\$^{*}$, mainly due to loss of tourism revenues and problems with electrical power generation (Johnston et al., 2000).

\section{Current hazard/risk at Mt. Ruapehu}

In 2004, Hurst et al. noted that volcanic eruptions are still the main hazard from Mt. Ruapehu, despite media coverage concentrating on possible crater lake outbreaks and lahars. Sector collapses, possibly inducing massive avalanches, are a hazard at least to adjacent populated areas (Thouret et al., 2006). Ash clouds can pose a high danger to aviation. Consequently, the air traffic over the North Island was halted briefly when Mt. Ruapehu started its unrest in 1995. However, due to intensive monitoring efforts and improved communication links between scientists and officials since 1995 (Hurst et al., 2004), the risk can be reduced if detailed monitoring is maintained.

The summit Crater Lake contains up to 10 million $\mathrm{m}^{3}$ of water and covers the historically active vent (Neall et al., 1999). Therefore the eruptions at Ruapehu are usually phreatomagmatic or phreatic unless the lake water is ejected due to volcanic activity. Temperature cycles in this hot acidic lake indicate volcanic activity underneath (Hurst et al., 1991). A lahar occurred on Mt. Ruapehu in the morning of 18 March 2007. The outbreak of the crater lake had been awaited since a minor hydrothermal eruption on 4 October 2006. According to press reports, the March 2007 lahar caused no immediate threats to life. Roads and a nearby rail track were closed preventively and the lahar went down Whangaehu river without any reported major incidents. Approximately $1.3 \cdot 10^{6} \mathrm{~m}^{3}$ of water and debris were released and

\footnotetext{
*New Zealand dollars
} 
the lake level dropped about $6 \mathrm{~m}$ (Ruapehu Alert Bulletin 01, 2007). Another minor eruption sent a lahar down the Whakapapa ski field on 25 September 2007 (Ruapehu Alert Bulletin 03, 2007). Ballistics were ejected as far as $1 \mathrm{~km}$ from the summit. The eruption was not followed by any further volcanic activity in the subsequent weeks.

Based on petrological data, Gamble et al. (2003) predict that future eruptions will not be very different from the eruptions in the past $2-3 \mathrm{ka}$, but they are not able to rule out the possibility of higher-scale events in the future.

\section{Seismicity at Mt. Ruapehu}

Volcanic earthquakes at Mt. Ruapehu were analysed and classified by Latter (1981). Compared to tectonic earthquakes, volcanic earthquakes have a typically lower frequency and can often be identified based on their waveform or spectrum. Sherburn et al. (1999) reviewed the seismicity of Ruapehu for 1971-1996 and noticed little changes within that time period. These authors estimated that the dominant frequency for both volcanic earthquakes and tremors is $2 \mathrm{~Hz}$. They pointed out that the seismicity during the eruption periods in 1995/96 differed from before that time (e.g. $1 \mathrm{~Hz}$ tremor in 1995/96 compared to $2 \mathrm{~Hz}$ and $7 \mathrm{~Hz}$ tremor in the years before).

To categorise the volcanic seismicity, Sherburn et al. (1999) sorted events into two groups: 1) volcanic (volcanic earthquakes and volcanic tremor) if magma or magmatic gases are involved and 2) volcano-tectonic (shallow and "all other"). Volcanic earthquakes with a magnitude of up to $M_{L} 4.0$ were detected.

Studies of the seismicity of Ruapehu led Sherburn et al. (1999) to the conclusion that some changes in the vent system occurred in the course of the 1995/96 eruptions. While $2 \mathrm{~Hz}$ tremors were commonly registered in the years before the eruptions, the disappearance of $2 \mathrm{~Hz}$ seismicity is explained with a disruption of a "resonator beneath crater lake". These authors suggest that the system will return to pre-eruption status 
within a few years, which agrees with the results of anisotropy monitoring studies (see Gerst and Savage, 2004, and Section 3.4.5).

\section{Monitoring Mt. Ruapehu}

This section briefly summarises the monitoring efforts at Mt. Ruapehu volcano. For an overview of monitoring activities throughout New Zealand see Hurst et al. (2004). A general description of volcano monitoring approaches is given by Scarpa and Tilling (1996). Geological and geophysical methods can measure and monitor several parameters and properties; a change over time of those parameters can be a hint for changes in processes within and underneath the volcano.

Probably the most obvious and oldest monitoring technique is visual observation. But the reliability is limited, as eruptions can occur without being preceded by major changes in "how the volcano looks". Nevertheless, Geological and Nuclear Sciences $(\mathrm{GNS})^{*}$ is operating a remote camera that can at least support the monitoring of Mt. Ruapehu. Visual recordings can be especially useful to correlate seismicity with volcanic activity.

Satellites can be used to monitor ground deformation within a certain area by using differential radar interferometry (DInSAR; differential interferometric synthetic aperture radar), but this method yields only snapshots at certain time intervals (Hurst et al., 2004). According to Miller et al. (2003), a geodetic real-time monitoring system using GPS receivers can be used to detect deformations of a sufficiently high amplitude ${ }^{\dagger}$ if the deformation occurs "fairly rapidly".

The connection between volcanic activity and ground deformation is similar to the connection between volcanic activity and the anisotropy measurements of this study

${ }^{*}$ GNS Science is a New Zealand research institute, formerly known as Institute of Geological and Nuclear Sciences.

$\dagger$ They deployed two GPS receivers to measure the relative position between them-and monitor changes of this position. For the circumstances of their study the signal detection threshold was estimated $27 \mathrm{~mm}$ for vertical and $11 \mathrm{~mm}$ for horizontal components. 
(see Section 2.4). Pressure build-up through magma ascension can cause ground deformation that is measurable on the surface.*

GNS and the Department of Conservation run an Eruption Detection System to achieve quick warnings for the lahar-endangered Whakapapa ski field (Hurst et al., 2004). A lahar is expected to reach the ski field just 3 minutes after the eruption that caused it (Houghton et al., 1987). The system was installed after the previous warning system failed to trigger when lahars occurred during the 1995 eruption sequence. The current eruption detection system estimates the probability of a lahar by considering seismic and acoustic data. It is described in detail by Sherburn and Bryan (1999).

Less than 30 minutes before the 1969 and 1975 eruptions, volcanic earthquakes were observed (Hurst et al., 2004). The eruption in 1996 was preceded by tremors, but precursors were noticed only three days prior to the eruption onset (Nakagawa et al., 1999). Volcanic earthquakes and tremors are now routinely monitored (see Section 1.2.4) and GeoNet stations are concentrated at volcanic centers at Tongariro (including Mt. Ruapehu) and Taranaki.

Temperature and chemical properties of the crater lake are routinely monitored. Despite the lack of obvious seismic precursors, the concentration of sulphate in the lake water increased several weeks before the onset of the eruptions in 1995 (Hurst et al., 2004). The lake water was ejected in the course of the 1995 eruptions, but the lake began refilling after activity ceased (Sherburn et al., 1999). Monitoring of the crater lake also contributes to the knowledge about activity underneath, but Sherburn et al. (1999) reported eruptions during both high and low temperature periods of the crater lake.

Tremor amplitudes changed only 10 hours before the 1995 eruption commencement. Eruptions in 1995 and 1996 were accompanied by tremor and volcanic earthquakes,

\footnotetext{
* Modelling of the displacement caused by overpressure in a vertical conduit revealed a vertical displacement of at least $68 \mathrm{~mm}$ for a build-up of $1 \mathrm{MPa}$ of pressure, which could have been detected even at $1 \mathrm{~km}$ distance from the summit (Miller et al., 2003).
} 
but phases of stronger tremor and larger numbers of volcanic earthquakes were also observed without any volcanic eruption (Sherburn et al., 1999).

A combination of several volcano monitoring techniques is necessary, as every single technique has its flaws and limitations. Seismicity provides valuable insights into the processes inside and underneath the volcano, but does not always correlate with eruption activity. For example, phreatic eruptions occurred in the 1980s without correlated seismicity (Sherburn et al., 1999).

In this study I investigate temporal changes in seismic anisotropy to possibly monitor stress changes and thereby monitor the volcanic processes at Mt. Ruapehu volcano. The connection between seismic anisotropy and volcanic activity is described in Section 2.4.

\subsection{GeoNet network}

Most data analysed in this study has been retrieved from the GeoNet seismological network. The nationwide distribution of the GeoNet stations is influenced by geological and geographical features. Whilst the distribution on the South Island is almost equidistant, stations on the North Island have a background distribution similar to the South Island but also have high-density local networks in the vicinity of the cities of Auckland and Wellington as well as around the volcanoes Mt. Taranaki and Mt. Ruapehu and in the Taupo Volcanic Zone. Figure 1.9 shows the distribution of GeoNet stations on the North Island. 


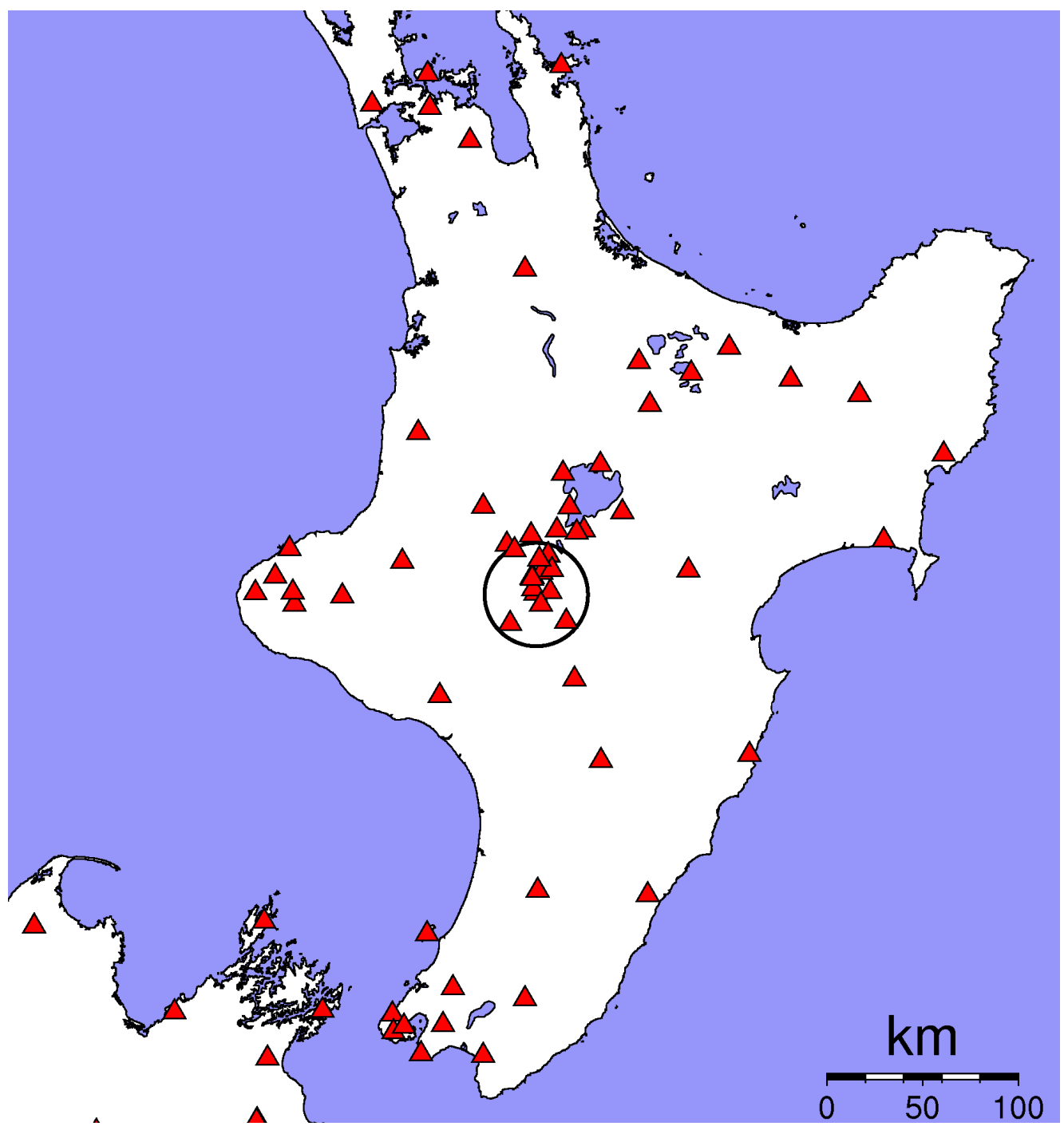

Figure 1.9.: GeoNet stations on the North Island of New Zealand. Note the increased density of stations at cities (Wellington, Auckland) and volcanoes (Mt. Taranaki, Mt. Ruapehu). The position of Mt. Ruapehu is indicated by a black circle with a radius of $25 \mathrm{~km}$. 


\section{Seismic anisotropy}

This chapter explains seismic anisotropy and its causes. The concept of shear wave splitting measurements to retrieve seismic anisotropy parameters is described in Chapter 3. The connection between anisotropy and volcanic activity is outlined and the results of some previous studies are summarised. Measurements of anisotropy in New Zealand are presented in Section 3.4 .4 (p.63).

\subsection{Definition of seismic anisotropy}

Anisotropy is the phenomenon in which a property or a process depends on direction. For example, optical anisotropy is known from crystal optics, as light propagation is dependent on the anisotropic properties of a crystal and the orientation of the beam of light relative to the symmetry axes of the crystal. Although there are numerous types of anisotropy, e.g. thermal conductivity anisotropy or electrical anisotropy, which can be relevant for hydrocarbon exploration, we focus on seismic anisotropy in this study. In the following text, anisotropy means seismic anisotropy, unless otherwise noted.

Seismic anisotropy describes the dependency of seismic wave speed on propagation direction or shear wave polarisation. In an anisotropic volume the wave speed is faster for propagation in one direction than it is in another, whereas in an isotropic volume the speeds are all independent of direction. Understanding of 
anisotropy makes it possible to derive information about the subsurface from seismological measurements on Earth's surface. As will be explained in the following sections, anisotropy is linked to certain properties of the rock, such as the state of stress, mineral composition and alignment, or deformation. Therefore measuring the anisotropic parameters is not to be seen as a final result, but as an intermediate step towards interpreting those parameters.

Recently, seismic anisotropy has been said to be a central feature of geophysics, especially when used for the exploration and exploitation of hydrocarbons (Thomsen, 2002). Ignored and even considered as noise in the past, anisotropy is now measured by exploration geophysicists to monitor or predict patterns of fluid flow (Armstrong et al., 1995). It is also seen as the most promising method of studying deep tectonics and dynamics of the earth (Babuška and Cara, 1991). Anisotropy is a common feature of crustal and mantle rocks and reflects present-day tectonic activities (Silver and Chan, 1991; Savage, 1999). Investigating anisotropy provides a better understanding of lithosphere-asthenosphere coupling and of possible links between crust and mantle structure (Fouch and Rondenay, 2006). There is a large variety of seismic anisotropy studies and there are numerous applications (Section 3.4). Sources can be active or passive, scales can be local, regional or global, and anisotropy can be measured in crust, mantle or core.

\subsubsection{Quantifying seismic anisotropy}

Seismic anisotropy is quantified with an anisotropy coefficient $k$ (Babuška and Cara, 1991):

$$
k[\%]=\frac{v_{\max }-v_{\min }}{\bar{v}} \cdot 100,
$$


or sometimes as shear wave anisotropy (SWA; Crampin, 1994), with

$$
\mathrm{SWA}=\frac{v_{\max }-v_{\min }}{v_{\max }} \cdot 100
$$

where $v_{\max }$ is the fast shear wave velocity, $v_{\min }$ is the slow shear wave velocity, and $\bar{v}$ is the average velocity, derived from $v_{\max }$ and $v_{\min }$ :

$$
\bar{v}=\frac{v_{\max }+v_{\min }}{2}
$$

so that there is a relation between $\mathrm{k}$ and SWA:

$$
k=\mathrm{SWA} \cdot \frac{v_{\max }}{\bar{v}} .
$$

Henceforth I will use $S_{1}$ and $S_{2}$ to refer to the shear wave velocities $v_{1}$ (or $v_{\max }$ ) and $v_{2}$ (or $v_{\text {min }}$ ) respectively. For crustal rocks, a velocity anisotropy of SWA $=1.5-4.5 \%$ is commonly observed (Crampin, 1994).

\subsection{What causes anisotropy?}

Seismic anisotropy can have several causes and interpretation of data can hence be difficult. Many properties of the rock mass can cause seismic anisotropy, including aligned fluid-filled or melt-filled cracks, aligned melt pockets, preferred orientation of crystals or alternating layers of contrasting seismic properties. Even if they are isotropic themselves, layers with different properties (velocities) can result in an anisotropic volume (Fig. 2.1, after Backus, 1962). However, measuring this 
anisotropy is scale-dependent. Only waves with a wavelength* much larger than the thickness of the layers will "sense" the anisotropy, because they sample the stack of layers as a whole (Backus, 1962; Armstrong et al., 1995). This means, that smallscale ordered heterogeneity causes large-scale anisotropy (Thomsen, 2002; Helbig and Thomsen, 2005). This layer-caused anisotropy is called transversely isotropic (TI), vertical transverse isotropy (VTI), or polar anisotropy and was the first described type of seismic anisotropy (Rudzki, 1898; Helbig and Thomsen, 2005).

Another cause of anisotropy is called shape preferred orientation (SPO), the presence of some geometrical order, caused by aligned microcracks, melt lenses, or even compositional heterogeneities. SPO is believed to contribute significantly to anisotropy beneath mid-ocean ridges and some portions of subduction zones, but is only a

Figure 2.1.: Isotropic layers can form an anisotropic volume. A rock built of alternating layers with different seismic properties would be anisotropic to waves with a wavelength larger than the thickness of the individual layers. This situation can be expected for sedimentary rocks. Particle motion across the layers is generally slower than within the layer plane.

try axis of this azimuthal anisotropy can

dip, but is often found to be subhorizontal, especially in the upper crust (Stein and

*The wavelength $\lambda$ of a seismic wave can be calculated from frequency $f$ and velocity $v: \lambda=$ $v / f$. To roughly calculate a lower estimate of the wavelength of shear waves near the surface at Mt. Ruapehu, we assume a shear wave velocity of $v_{s}=1.6 \mathrm{~km} / \mathrm{s}$ and a frequency of $10 \mathrm{~Hz}$, resulting in a wavelength of $160 \mathrm{~m}$. Even with these conservative estimations (shear wave velocity is approximately $2.5 \mathrm{~km} / \mathrm{s}$ below the uppermost layers at Ruapehu and mean frequencies were 4.1 for shallow and $2.4 \mathrm{~Hz}$ for deep events in 2002 data in the study of Gerst, 2003) the wavelength is several magnitudes higher than the dimension of a microcrack. 
Wysession, 2003). There are mainly two mechanisms to cause this type of anisotropy: Lattice preferred orientation of minerals and the preferred alignment of microcracks.

\subsubsection{Possible causes of mantle anisotropy}

Seismic anisotropy in the earth's mantle has long been observed in geophysical studies (e.g. in refraction profiles; Hess, 1964). Nicolas and Christensen (1987) explain these observations with lattice preferred orientation (LPO) of minerals. The mechanical properties and behaviour of the upper mantle are governed by olivine minerals (Mainprice et al., 2005).

Olivine minerals make up about $70 \%$ of the upper mantle (Ismal and Mainprice, 1998) and are anisotropic.* Ismal and Mainprice conclude that shear wave anisotropy is affected by the distribution of the crystallographic axes. Table 2.1 lists anisotropy values for some mantle minerals.

\begin{tabular}{|l||c|c|}
\hline mineral & $\begin{array}{c}\text { P-wave } \\
\text { anisotropy [\%] }\end{array}$ & $\begin{array}{c}\text { S-wave } \\
\text { anisotropy [\%] }\end{array}$ \\
\hline Olivine & 24.6 & 22.3 \\
Orthopyroxene (bronzit) & 16.4 & 15.6 \\
Garnet (pyrope) & 0.6 & 1 \\
\hline
\end{tabular}

Table 2.1.: P- and S-wave anisotropy for mantle minerals. Anisotropy is given in $k \%$ as defined in Equation 2.1, data are from Babuška and Cara (1991) and references therein.

If anisotropic crystals are randomly oriented, the rock mass they form is not anisotropic, as the small-scale anisotropy of the single crystals will be averaged over the large scale of the rock. For a large rock volume to be anisotropic, the single olivine crystals have to be oriented (LPO, see Fig. 2.2). In the mantle, this preferred orientation is interpreted to be the result of dislocation creep deformation of the

*Ismal and Mainprice (1998) find 4.0-21.4\% anisotropy for P-waves and 3.3-17.4\% for S-waves, with means of 12.2 and $9.4 \%$ respectively in 110 samples of olivine from various environments. 

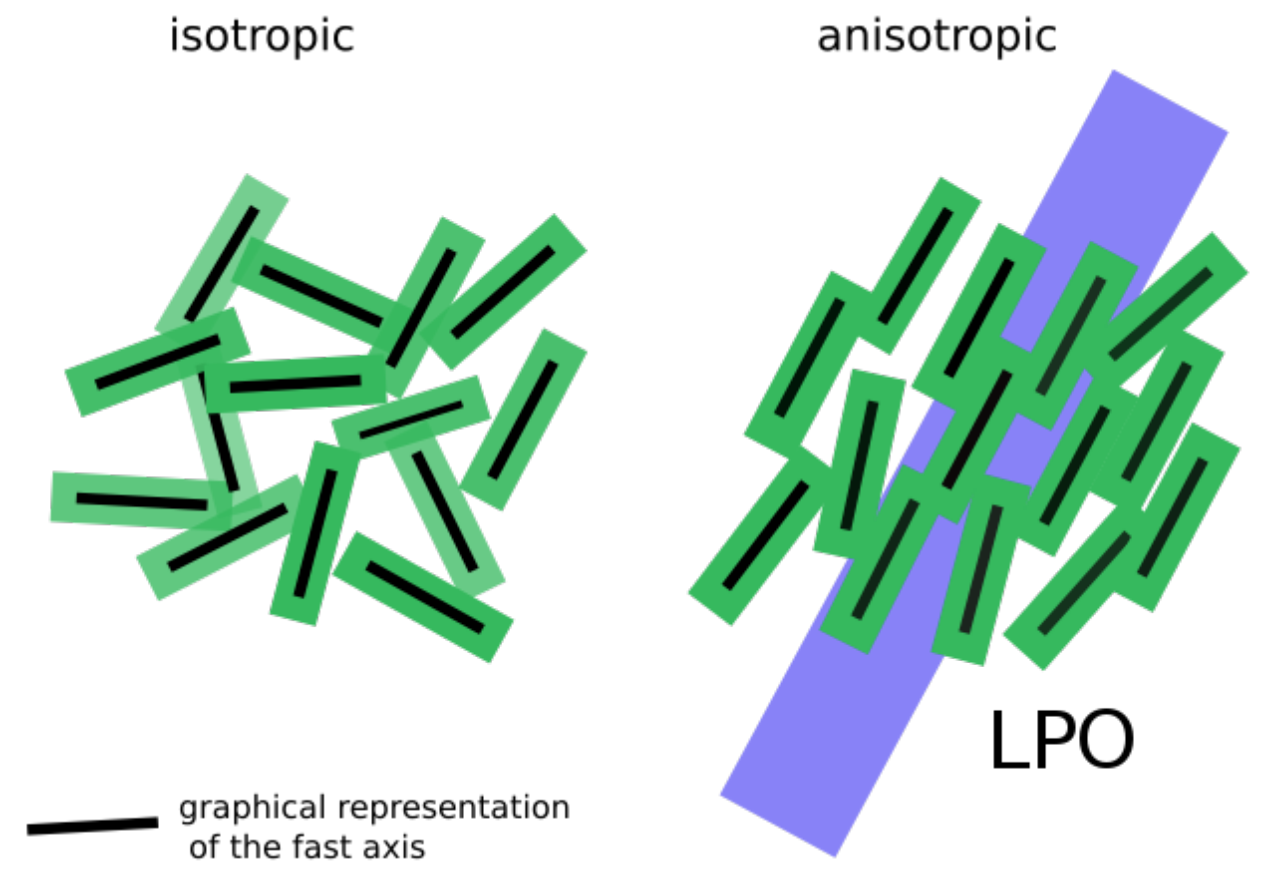

Figure 2.2.: Randomly oriented single crystals result in an isotropic volume (left), while lattice preferred orientation causes anisotropy (right, the blue bar indicates the resulting fast orientation)

olivine aggregates, which is a common process in the earth's mantle (Nicolas and Christensen, 1987; Karato and Wu, 1993).

The observed decrease of anisotropy in the deep upper mantle below $250 \mathrm{~km}$ was often interpreted as a regime change from dislocation creep to diffusion creep, but high-P laboratory experiments on olivine indicate a change in slip direction in a still “dislocation creep dominated" environment (Mainprice et al., 2005).

For a common dry upper mantle the fast $a$-axis of olivine is parallel to the lineation direction (Silver, 1996). Therefore the fast direction of the medium should be parallel to the lineation direction (the direction of flow). Hence the notation of strain-induced lattice preferred orientation (Hess, 1964; Silver and Chan, 1991). The alignment of olivine crystals provides insights into the mantle strain field and mantle deformation. Mantle anisotropy is not always caused by lattice preferred orientation. Walker et al. (2004) rule out extension-induced LPO as a cause of anisotropy at the East African 


\begin{tabular}{|l||c|c|}
\hline mineral & $\begin{array}{c}\text { P-wave } \\
\text { anisotropy [\%] }\end{array}$ & $\begin{array}{c}\text { S-wave } \\
\text { anisotropy [\%] }\end{array}$ \\
\hline Quartz & 26.5 & 40.1 \\
Calcite & 32.7 & 56.7 \\
Feldspar (microcline) & 46.0 & 79.4 \\
Feldspar (oligoclase) & 31.4 & 55.7 \\
Diopside & 21.4 & 20.3 \\
Hornblende & 27.2 & 30.8 \\
Muscovite & 57.9 & 84.7 \\
Biotite & 59.7 & 116.3 \\
\hline
\end{tabular}

Table 2.2.: P- and S-wave anisotropy for crustal minerals. Anisotropy is given in $k \%$ as defined in Equation 2.1, data are from Babuška and Cara (1991) and references therein.

rift - a clearly extensional regime - and explain their observations with the presence of fossil anisotropy, plume flow and aligned magma lenses.

\subsubsection{Causes of crustal anisotropy}

The mineral composition of the earth's continental crust differs from that of the mantle. Compared mantle minerals (Table 2.1) crustal minerals are just as anisotropic, if not more (Table 2.2). But while lattice preferred orientation governs the anisotropy of mantle rocks, this is not necessarily true for crustal rocks. A granite for example is mainly made out of feldspar, quartz and mica, all of which have high anisotropy coefficients (Table 2.2). But (crack-free) granite rocks have a low anisotropy (Birch, 1960). This can be explained with two causes: First, granite rocks seldom consist of oriented minerals.* Second, even sheared granitic rocks with oriented minerals have low anisotropy coefficients, because quartz and feldspars are not oriented according to their "fast" and "slow" axes (Babuška and Cara, 1991). Therefore, even LPO does not affect granites in the crust as strongly as mantle rocks containing other minerals.

\footnotetext{
${ }^{*}$ It is therefore no surprise that metamorphic rocks (especially schists) are usually more anisotropic than plutonic granite.
} 
Continental crust is more heterogeneous than mantle rocks or oceanic crust. It forms mountains, valleys, and basins and contains sedimentary, magmatic and metamorphic rocks. Composition can vary in regional and local scales, while tectonic processes, such as faulting or uplift can alter the crustal rocks. Structural features itself can cause anisotropy (e.g. faults; Zinke and Zoback, 2000). It is no surprise that schists and layered sediments are anisotropic (recall Fig. 2.1), and the lower crust can be highly anisotropic because of schists, but LPO alone can often not sufficiently explain the observed crustal anisotropy. Several studies of receiver functions indicate a large degree of crustal anisotropy (e.g. Savage, 1998; Wilson et al., 2004; Horspool et al., 2006). Large faults were found to influence the anisotropy locally, as has been reported at San Andreas Fault (Cochran et al., 2003) or at the North Anatolian Fault (Peng and Ben-Zion, 2004).

\subsubsection{Microcracks}

Microcracks are cracks that are not longer than grain size (Broberg, 1999). They are usually cracks between grains - not within them. Crack sizes typically range from $10^{-6} \mathrm{~m}$ to a few metres (Crampin, 1994), whereas microcracks are by definition not larger than grain sizes (not more than a few $\mathrm{cm}$ ). As a first estimate, microcracks can be assumed to be penny-shaped or have a similarly simple geometry (Crampin, 1999). The crack normal is therefore perpendicular to the flat surface of such a penny-shaped form.

Nearly all crustal rocks contain microcracks and in the earth's crust they are typically fluid-filled (Babuška and Cara, 1991). In the context of this study, the term "microcracks" is used to cover intergranular and typically fluid-filled small cracks. The connection between microcracks and anisotropy is essential for the monitoring of anisotropy and stress. While preferred orientation of minerals takes place in geological timescales, microcracks can open and close in much shorter time (see Section 2.3). 
The oriented alignment of cracks can only cause anisotropy if the fluid pressure is sufficient to keep the cracks opened. But for the uppermost $400 \mathrm{~km}$ of the mantle, stress-aligned melt films could have a similar effect as microcracks have in the crust (Crampin and Peacock, 2005).
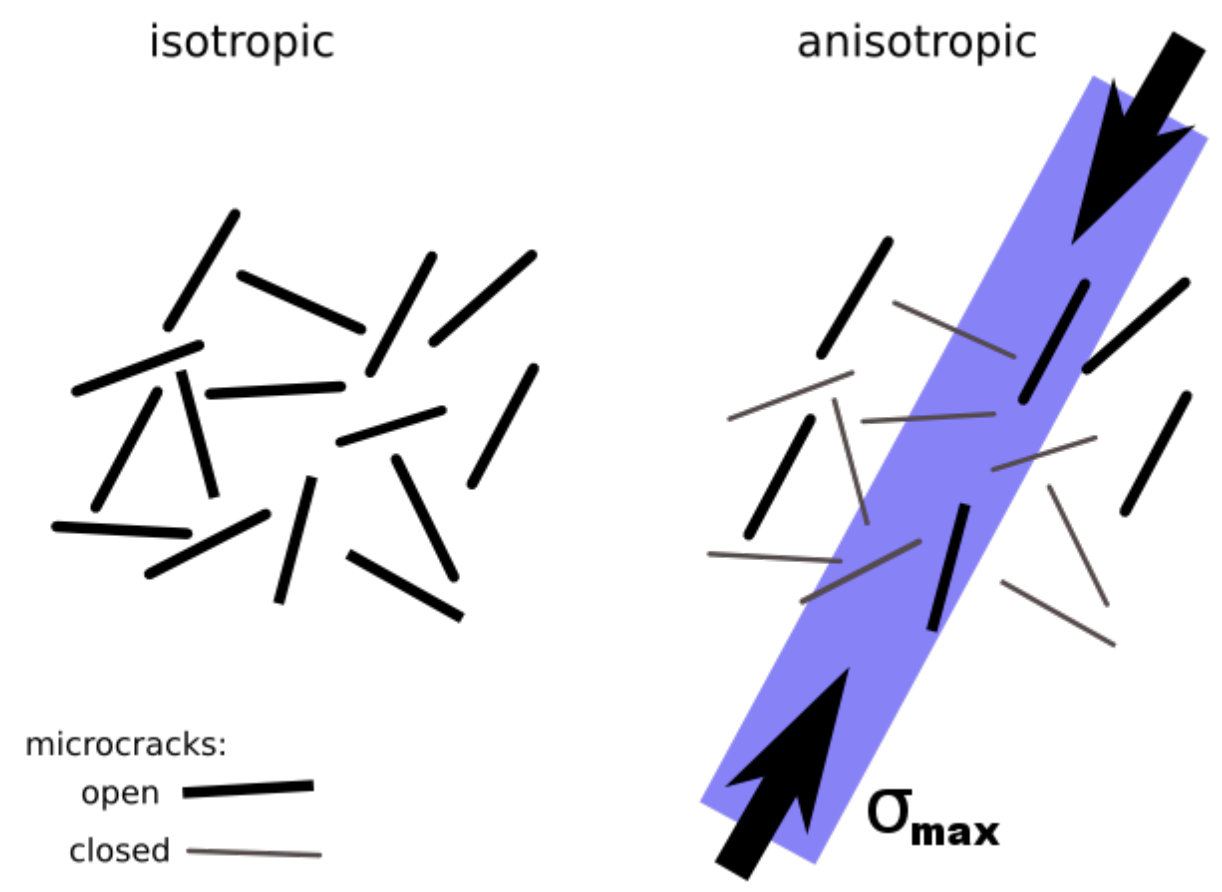

Figure 2.3.: Randomly oriented microcracks do not cause significant anisotropy (left), while microcracks with preferred orientation do (right, the blue bar indicates the resulting fast orientation). Arrows $\left(\sigma_{\max }\right)$ represent maximum compression, microcracks that are not subparallel to $\sigma_{\max }$ are closed (or cannot open). In a real rock the variance of single cracks is expected to be up to $\sim 30-40^{\circ}$ (depending on the differential stress), but the average orientation is subparallel to the maximum horizontal stress (Crampin and Zatsepin, 1997). As indicated by this figure, single cracks are not literally rotated or aligned, but the average orientation of open microcracks is.

If cracks are randomly distributed, no anisotropy is caused by those cracks. But if cracks are aligned, the rock is anisotropic, while the rock mass itself (without the cracks) might be isotropic. This is illustrated by the two schematic rock volumes shown in Figure 2.3. The alignment of microcracks has been described as extensive dilatancy anisotropy (EDA, see Crampin et al., 1984; Crampin, 1987). Crack alignment is not literally an alignment, as a single crack is not rotated 
or aligned, but more a selective process, where cracks of a certain orientation are opened (or remain opened) while others remain closed (or are closed). Consequently, "crack alignment" hereafter refers to this selective process, which Crampin and Zatsepin (1997) name "stress-induced alignment of crack distributions". The presence of two distinct sets of crack orientations is possible*(e.g. Liu et al., 1993).

There is a critical angle that separates open and closed microcracks. This angle is a function of the differential stress, and the variance of single cracks can still be 30-40 degrees (Figure 3 of Crampin and Zatsepin, 1997). As a consequence, only the average orientation of the microcracks in a rock volume is subparallel to the orientation of maximum horizontal stress. This leads to an hexagonal symmetry of the rock volume.

However, a seismic wave typically has a wavelength much larger than the dimensions of microcracks and seismological methods will therefore sample an average crack orientation. This is in agreement with the numerical modelling of microcrack behaviour in other studies (e.g. Tod, 2002), but some ambiguities about the connection between crack alignment and stress field remain. Laubach et al. (2004) point out that chemical processes (e.g. precipitated cements that seal a certain crack configuration) can prevent crack distributions from aligning in some environments.

Anisotropy usually decreases with depth (Bokelmann and Harjes, 2000). In the upper crust, crack-induced anisotropy is normally restricted to depths down to 10$15 \mathrm{~km}$. At greater depths the cracks are either closed (Nur and Simmons, 1969) or horizontally aligned without large effects on waves approaching from underneath (Kaneshima et al., 1988). Observations of anisotropy restricted to the shallow upper

\footnotetext{
*For shear wave splitting measurements, two sets of crack orientations are expected to yield an average fast orientation of both crack orientations (see Section 3.2), depending on crack densities of the two subsets (Liu et al., 1993; Elkibbi et al., 2005)
} 
crust can be interpreted to support the model of crack-induced crustal anisotropy (e.g. for an area in southern Japan; Kaneshima et al., 1988).

Almost all crustal rocks contain microcracks, with a range of typical crack densities $0.015 \leq \varepsilon \leq 0.045$ for intact rock and $\varepsilon>0.045$ for heavily fractured rock, where

$$
\varepsilon=N a^{3} / V
$$

with $N$ being the number of cracks in a volume $V$, and $a$ being the crack radius (Hudson, 1981). Crack density and velocity anisotropy for shear waves are related, and at least for small crack densities there is a rule of thumb:

$$
\mathrm{SWA}=100 \cdot \varepsilon
$$

where SWA is shear wave velocity anisotropy (Equation 2.2) and crack density $\varepsilon$ is defined as in Equation 2.5 (Crampin, 1994). Besides this empirical estimation there are also analytical approaches to relate crack density to seismic anisotropy (e.g. Hudson, 1981; Thomsen, 1995). To estimate the crack density from splitting measurements, the following formula can be used:

$$
\varepsilon=\frac{7}{8} \cdot \frac{v_{s} \delta t}{L}
$$

where $v_{s}$ is shear wave velocity without cracks, $\delta t$ is the delay time and $\mathrm{L}$ is the path length of the wave through the anisotropic medium (Hudson, 1981; Kuehler et al., submitted). This estimation is only valid if the anisotropy is mainly caused by microcracks (Cochran et al., 2003), which is not always clear.

For example, contrary results were obtained from shear wave splitting measurements in Brazil, where anisotropy was interpreted to be palaeo-stress induced (by foliation of the rock) and fast orientations were not parallel - as would be expected 
for anisotropy caused by aligned microcracks--, but perpendicular to the orientation of maximum horizontal stress (do Nascimento et al., 2002) and about $50^{\circ}$ offset in another study (do Nascimento et al., 2004a).

The interpretation of do Nascimento et al. (2002) was debated by Crampin (2004), who claimed that a fast polarisation perpendicular to the maximum horizontal stress can also be caused by a high pore pressure-induced $90^{\circ}$-flip (see Section 3.2.2). However, the palaeo-stress hypothesis is in agreement with other studies (see do Nascimento et al., 2002, 2004b, and references therein). The results of do Nascimento et al. (2004a) also indicate that the anisotropy in this area is controlled by tectonic fabric.*

Thus, the hypothesis of microcrack-induced anisotropy can explain many observations, but other possible causes (depending on the geological setting) must be considered in the analysis of shear wave splitting measurements.

\subsection{Temporal changes in anisotropy (theory)}

To enable scientists to monitor temporal changes in anisotropy, the processes that induce these changes must work in reasonable timescales. In 1980, Crampin et al. observed shear wave splitting consistent with aligned microcracks, where the alignment of existing cracks could explain various precursory phenomena before earthquakes. Observations at the Northern Anatolian Fault showed shear wave splitting for almost every analysed earthquake. Other possible explanations, such as isotropic discontinuities, source effects, or mixing of Love- and Rayleigh waves could be ruled out. The effects of aligned cracks were believed to be strong enough to account for the observed splitting, even in the heterogeneous regions in the proximity of the studied site. It was concluded that stress can be monitored with shear wave splitting measurements.

*The study of do Nascimento et al. (2002) analysed seismic anisotropy in the João Câmara area, NE Brazil. The other study (do Nascimento et al., 2004a) was performed in the Açu dam area, which is located $150 \mathrm{~km}$ west of João Câmara and has a "similar tectonic setting." 
This led eventually to the development of the anisotropic poroelasticity (APE) model (Zatsepin and Crampin, 1997), which describes the behaviour of microcracks when pressure changes occur.* According to the APE-model, the deformation of a crack volume depends on the stress in the direction of the crack normal, and therefore on the orientation of the crack in the stress field. Fluid migration between adjacent cracks is the mechanism that allows cracks to open and close under the influence of a changing stress field, and

$$
t^{c} \propto \frac{5}{N a^{3} / V} \frac{\left(\sigma^{c} / \mu\right) a^{2} \eta}{k \Delta \sigma}
$$

gives the characteristic time $t^{c}$ of this process, where $N$ is the number of cracks in volume $V, a$ is the crack radius, $\sigma^{c}$ is the critical stress for crack closure, $\mu$ is shear modulus of the matrix, $\eta$ is the viscosity of the pore fluid, $\Delta \sigma$ is the differential stress, and $k$ is a permeability (modified after Crampin, 1994; Zatsepin and Crampin, 1997). Zatsepin and Crampin give $t^{c}=10^{4} s$ as an upper limit for typical crustal rocks with low-permability $\left(10^{-9}\right.$ darcy) and for a relatively low differential stress of $1 \mathrm{MPa}$. These authors expect fluid diffusion to be even faster at depth (because higher temperature increases permeabilities and decreases fluid viscosity) and conclude that crack-alignment and therefore temporal variations in anisotropy can be expected after times of the same order as $t^{c}$.

The unequivocal difference in timescales helps to distinguish two possible causes of anisotropy. The quick, almost instantaneous reaction of crack alignment to changes in the stress field allows us to observe temporal variations in anisotropy, unlike the lattice preferred orientation of minerals by material flow, which takes place in much longer timescales. This allows a set of new anisotropy monitoring applications, such as monitoring of hydrocarbon reservoirs during production, and generally the moni-

\footnotetext{
*The APE model suggests that cracks are on the edge of fracture criticality and are spaced closely, so they behave as a critical system, sensitive to small changes (Crampin, 1994).
} 
toring of in-situ stress (e.g. Crampin and Chastin, 2003). The shear wave splitting phenomenon (Section 3.2) allows a simple in-situ measurement of crack-alignment and stress-field with a seismological approach.

\subsection{Stress: a connection to volcanic activity}

Crack alignment, one of the causes of anisotropy (explained in Section 2.2), is the result of a stress field applied to rock. It is suitable for monitoring, because cracks align almost instantaneously when the stress field changes (see Section 2.2.3).

Emplacement of a vertical magmatic intrusion (dike) is expected to be parallel to the orientation of the maximum horizontal stress $\sigma_{H}$, because opening of a dike against the stress field would face the least resistance against the smallest horizontal stress $\sigma_{h}$. Analogous to the alignment of microcracks, this would cause dikes to be aligned parallel to the orientation of the maximum horizontal stress. Alignment of volcanic dikes according to the stress field has been observed in the field and is in agreement with stress field calculations and numerical modelling so that dikes are used as common stress indicators (Nakamura, 1977; Nakamura et al., 1977; Rubin, 1995; Gudmundsson, 2002, 2003).

Volcanic dikes are able to reorient the local stress field and this is assumed to be a common process among volcanoes (Parsons and Thompson, 1991). When magma is ascending, an overpressure is imposed on the walls of the feeding system (Russo et al., 1996). Changes in stress orientation that support the model of an overpressurised dike system were discovered at Unzen volcano in Japan (Umakoshi et al., 2001). Furthermore, before an eruption in 1992 at Mt. Spurr volcano in Alaska, the maximum horizontal stress in the vicinity of the volcano was perpendicular to the orientation of regional maximum horizontal stress. After the eruption it was parallel, 
but eventually it was perpendicular again, probably because a magmatic intrusion overpressured the system again (Roman et al., 2004).

The connection between volcanic activity and anisotropy is illustrated in the flowchart shown in Figure 2.4.

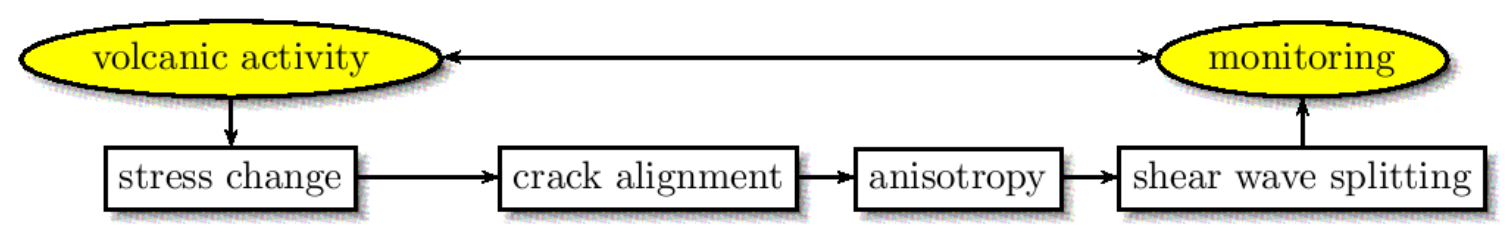

Figure 2.4.: Connection between volcanic activity, stress changes and anisotropy. Volcanic activity, such as crustal magma emplacement can cause an overpressurising of the magmatic dike system. This can change the orientation of the stress field locally into a different one than the regional. Reorientation of the stress field can realign the distribution of microcracks in the earth's crust and therefore change the anisotropy of the medium. This can be measured and monitored with the shear wave splitting method.

\subsubsection{Dike pressurising at Mt. Ruapehu}

There is evidence at Mt. Ruapehu that the subsurface picture is more complicated than the simplification of a single magma chamber feeding material into a single conduit. As mentioned in Section 1.2.3, there are hints for the existence of several fossil vents. Another indicator for a more complicated situation is the suggestion of Nakagawa et al. (1999) that the andesites produced by the 1995 eruptions are a mixing product of two distinct magmas.

Dike pressurising has been found to induce sufficiently large pressure changes to align microcracks in the vicinity of Mt. Ruapehu (Gerst, 2003). To re-align microcracks, an overpressure that is sufficiently high to overprint the regional stress field has to be induced and the effective stress perpendicular to the dike must be larger than the previously larger horizontal stress:

$$
\sigma_{\text {dike }}+\sigma_{h}>\sigma_{H}
$$



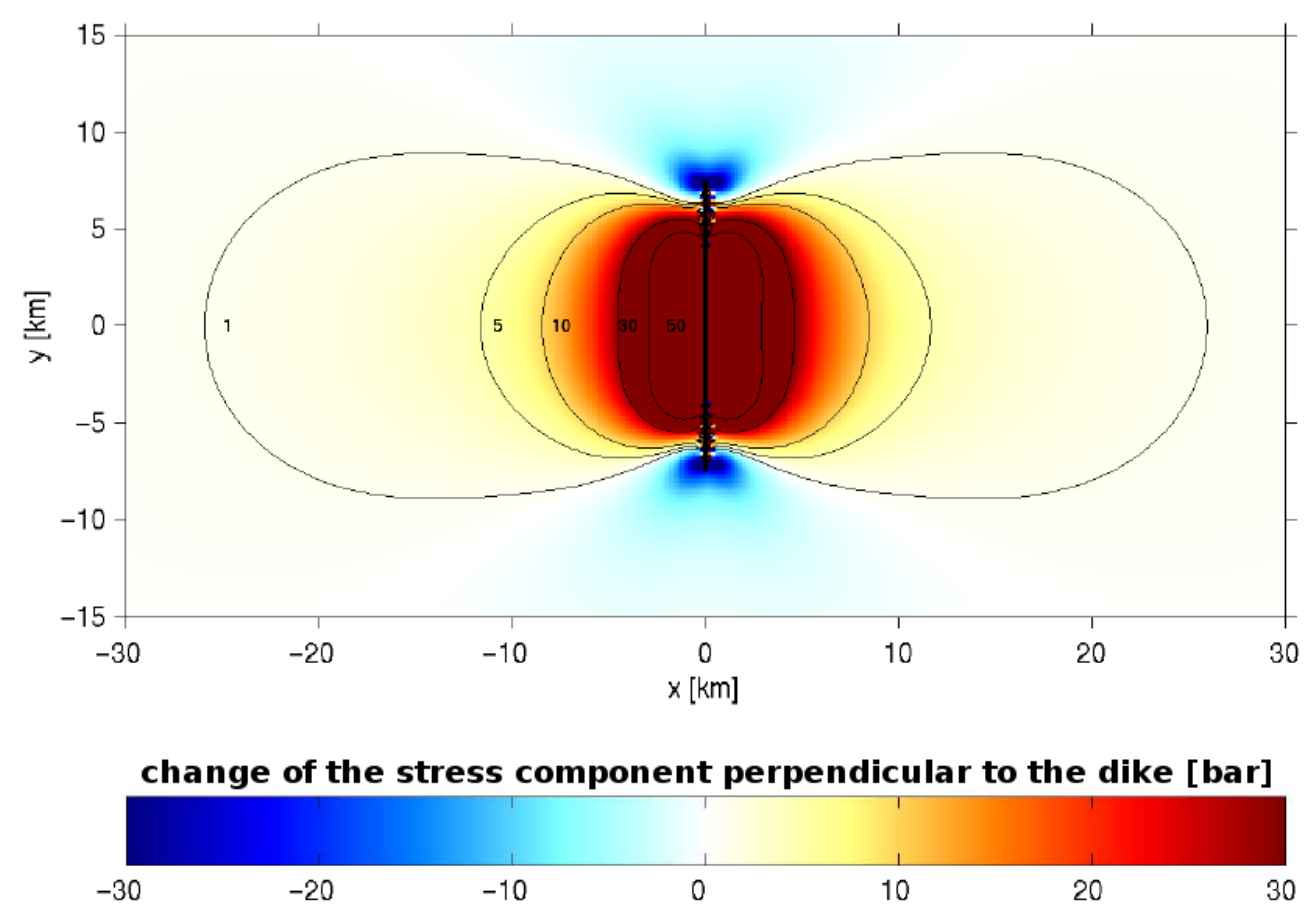

Figure 2.5.: Modelled stress changes caused by a magmatic dike. Geometry of the dike for this numerical model is $15 \mathrm{~km}$ length, $6 \mathrm{~km}$ height at $2-8 \mathrm{~km}$ depth and a thickness of $1 \mathrm{~m}$. The change of stress perpendicular to the dike is shown in bar, for a map view of a plane at $5 \mathrm{~km}$ depth. Source: Gerst (2003), Modelling software: Toda et al. (1998)

when the dike is aligned parallel to $\sigma_{H}$ and the overpressure $\sigma_{\text {dike }}$ is applied to the dike wall. A numerical model of stress changes perpendicular to a dike (Gerst, 2003; Toda et al., 1998) indicates that a pressurised dike* can cause stress changes of $1 \mathrm{MPa}$ as far as $10 \mathrm{~km}$ from the dike and changes of $5 \mathrm{MPa}$ within $3 \mathrm{~km}$ (see Figure 2.5).

The differential stress for polygenetic volcanoes like Mt. Ruapehu is usually small (Takada, 1994). A calculation after Zoback and Townend (2001) yields estimated values of $130 \mathrm{MPa}$ for $\sigma_{V}$ and $70 \mathrm{MPa}$ for $\sigma_{h}$, if a density of $2.700 \mathrm{~kg} / \mathrm{m}^{3}$ and a hydrostatic fluid pressure are assumed. The maximum horizontal stress $\sigma_{H}$ is not easily calculable, but is possibly only little larger than $\sigma_{h}$ (Gerst, 2003). The orientation of the stress field at Mt. Ruapehu is discussed in Section 3.4.4.

${ }^{*}$ Change of stress is calculated for a dike of $15 \mathrm{~km}$ length, $6 \mathrm{~km}$ height, $1 \mathrm{~m}$ opening at the centre, and tapered endings. An elastic half space with a Poisson's ratio of 0.25 and a Young's modulus of $40 \mathrm{GPa}$ are chosen. The depth region of the dike is assumed to be $2-8 \mathrm{~km}$, based on studies of seismicity (Latter, 1981). The dimensions of the dike are chosen to roughly represent the erupted volume for the 1995/96 eruptions $\left(0.05 \mathrm{~km}^{3}\right)$ at Mt. Ruapehu. 


\section{Measuring anisotropy}

Geophysicists can measure anisotropy with seismological methods because of its effects on seismic waves. One such effect is the splitting of shear waves. The following chapter will provide an overview of anisotropy and shear wave splitting measurements, while the method used in this study is presented in the Chapter 4 in greater detail.

A calcite crystal placed on a textbook page will reveal the anisotropy of the crystal, as the light beams will split and the letters are visible twofold, with an offset. This birefringence (or double refraction) phenomenon was discovered in the 17 th century (Bartholin, 1669). A seismic shear wave that travels through an anisotropic medium also splits into a fast and a slow component; this phenomenon is called shear wave splitting.

\subsection{Wave equation for anisotropic media}

The equations for wave propagation in anisotropic media are derived below. Derivations of wave-equations and velocity equations follow Babuška and Cara (1991), Thomsen (2002) and Stein and Wysession (2003), but notation has been adjusted for consistency and summation convention is used for simplicity. To answer the question Why do shear waves split?, we derive and analyse a wave equation for anisotropic 
media and use it to describe the propagation of a seismic wave through an anisotropic medium.

For a homogeneous and isotropic medium the three-dimensional wave equation is

$$
\frac{1}{c_{2}} \frac{\partial^{2} u_{i}}{\partial t^{2}}=\frac{\partial^{2} u_{i}}{\partial x_{i}}
$$

or even shorter in Newton's notation: $\ddot{u}=c^{2} u^{\prime \prime}$, where $c$ is the wave's phase velocity and $u(x, t)$ is the vector of the particle motion with components $u_{i}, \ddot{u}$ is the second time derivative of $u$, and $u^{\prime \prime}$ is the second spatial derivative. But for seismic waves we need to derive a more complex wave equation that considers the elastic properties of a "real medium".

Elastic behaviour and symmetry of a medium are described by the elasticity tensor* c with components $c_{i j k l}$. Hooke's law relates stress to strain for elastic deformations. Stress and strain are described by the second-rank $(3 \times 3)$ tensors $\sigma$ and $\varepsilon$ respectively and they are connected by the fourth-rank $(3 \times 3 \times 3 \times 3)$ elasticity tensor as follows:

$$
\sigma_{i j}=c_{i j k l} \varepsilon_{k l},
$$

with $i, j, k, l=1,2,3$. The stress and strain tensor are both symmetric, hence follow $c_{i j k l}=c_{j i k l}$ and $c_{i j k l}=c_{i j l k}$ and through strain energy storage constraints $c_{i j k l}=c_{k l i j}$. This allows us to express the 81 components of the elasticity tensor with "only" 21 independent components. Now, if we insert Hooke's law (Equation 3.1) into the right side of the equation of motion for a three-dimensional continuum (after Newton):

$$
\begin{aligned}
\rho \frac{\partial^{2} u_{i}}{\partial t^{2}} & =\frac{\partial \sigma_{i j}}{\partial x_{j}} \\
& =c_{i j k l} \frac{\partial \varepsilon_{k l}}{\partial x_{j}}
\end{aligned}
$$

\footnotetext{
${ }^{*}$ sometimes also named stiffness tensor
} 
with $\rho$ being the density of the medium, we get an equation that can be further transformed, using the definition of small strain:

$$
\varepsilon_{k l} \equiv \frac{1}{2}\left(\frac{\partial u_{k}}{\partial x_{l}}+\frac{\partial u_{l}}{\partial x_{k}}\right)
$$

so that we obtain a wave equation (which contains the elasticity tensor), for a homogeneous but possibly anisotropic medium:

$$
\rho \frac{\partial^{2} u_{i}}{\partial t^{2}}=c_{i j k l} \frac{\partial^{2} u_{l}}{\partial x_{j} \partial x_{k}}
$$

We want to investigate the effect of anisotropy on wave propagation. As can be seen in Equation 3.2, the elasticity tensor governs the propagation of an elastic wave, thus also its velocity. The propagation of a plane wave is described by its displacement vector:

$$
\vec{u}(\vec{x}, t)=\vec{a} f\left(t-\frac{\vec{n} \cdot \vec{x}}{c}\right)
$$

where $c$ is phase velocity, $\vec{x}$ is the particle coordinate, $\vec{n}$ is the unit vector pointing into propagation direction, $\vec{a}$ is a vector which describes amplitude and polarisation (this is important for the eigenvalue problem we will face soon), and $f$ is a function describing the particle motion, such as

$$
f(\vec{x}, t)=\exp \left[i \omega\left(t-\frac{\vec{n} \cdot \vec{x}}{c}\right)\right],
$$

modified from Crampin (1984). However, if we insert Equation 3.3 into the wave equation (3.2), we get three equations that are independent of $f$, because twofold differentiation of $f$ with respect to $x$ and $t$, following the chain rule, allows us to omit 
$f^{\prime \prime}$ on each side of the three equations:

$$
\begin{aligned}
\rho a_{i} f^{\mu \prime} & =c_{i j k l} a_{l} \frac{n_{j}}{c} \frac{n_{k}}{c} f^{\mu \prime} \\
\Longrightarrow \quad c^{2} a_{i} & =\frac{c_{i j k l} n_{j} n_{k}}{\rho}, \quad i=1,2,3 .
\end{aligned}
$$

The terms on the right side of this equation can be expressed with a matrix, the Christoffel matrix (Christoffel, 1877), which is symmetric:

$$
m_{i l}=c_{i j k l} n_{j} n_{k} / \rho,
$$

so we finally get

$$
m_{i l} a_{l}=c^{2} a_{i}
$$

an eigenvalue problem which is satisfied by the three mutually orthogonal eigenvectors $\vec{a}_{1}, \vec{a}_{2}$ and $\vec{a}_{3}$ and their corresponding eigenvalues $c_{1}^{2}, c_{2}^{2}$ and $c_{3}^{2}$. The square root of each eigenvalue represents the phase velocity of one type of wave.

\subsection{Shear wave splitting}

One way of investigating anisotropy is the measurement of shear wave splitting which is usually done with seismic stations on the earth's surface. Shear wave splitting occurs when an S-wave propagates through anisotropic material. For a particular propagation direction, shear waves in such material can only be polarised in two orientations (fast and slow), corresponding to the two eigenvectors of the Christoffel matrix (Equation 3.6). A shear wave with an initial intermediate polarisation will split into two distinct orthogonal waves that propagate with their own distinct speed. Therefore, polarisation of the S-waves is no longer determined by the source, but is now dependent on the properties of the medium and the propagation direction. 
The phase velocity of seismic body waves is controlled by the elastic parameters of the elasticity tensor. For an isotropic medium, the elasticity tensor contains only two independent parameters and yields the following Christoffel matrix*:

$$
m_{i l}=c_{i 11 l} \frac{n_{1} n_{1}}{\rho}=\frac{1}{\rho}\left(\begin{array}{ccc}
\lambda+2 \mu & . & . \\
. & \mu & . \\
\cdot & \cdot & \mu
\end{array}\right) \text {, }
$$

for a plane wave propagating in $x_{1}$ direction, so that $\vec{n}=\vec{e}_{1}$. As expected, for three eigenvectors we get two distinct eigenvalues, $c_{1}^{2}=\lambda+2 \mu$ and $c_{2}^{2}=\lambda_{3}=\mu$. The corresponding wave speeds are

$$
\begin{aligned}
P_{1} & =\sqrt{c_{1}^{2}}=\sqrt{\frac{\lambda+2 \mu}{\rho}} \\
\text { and } \quad S_{1}=S_{2} & =\sqrt{c_{2}^{2}}=\sqrt{\frac{\mu}{\rho}},
\end{aligned}
$$

which represent a $\mathrm{P}$-wave that is polarised parallel to the propagation direction and an S-wave that is polarised perpendicular to propagation direction. Both waves are arbitrarily polarised within their plane, so that the polarisation is dependent on the source. In this isotropic medium the shear waves will not split, because the velocity for all their polarisation directions is identical. This is different in an anisotropic medium.

Anisotropy with a hexagonal symmetry occurs in the case of alternating layers (Figure 2.1), aligned cracks and lattice preferred orientation, if we assume that in the latter only the fast axis is oriented, while the two slow axes are distributed randomly (or vice versa). The case of vertical transverse isotropy (as induced by layered material, e.g. Figure 3.1) allows a clear illustration of the shear wave splitting

*Independent of propagation direction for the isotropic case. Compare with Equation 3.5, where the propagation direction $\vec{n}$ does affect the wave propagation for anisotropic systems. 
phenomenon. In this case, shear wave splitting occurs, when the wave propagates approximately horizontally (the split waves are then known as SH and SV).

No splitting only occurs in the case of propagation along the symmetry axis. From the elasticity coefficients for a hexagonal symmetry (Babuška and Cara, 1991) follows the Christoffel matrix:

$$
\begin{array}{rlr}
m_{i j}=\frac{1}{\rho}\left(\begin{array}{ccc}
A & \cdot & \cdot \\
\cdot & N & \cdot \\
\cdot & \cdot & L
\end{array}\right), & n=\left(\begin{array}{l}
1 \\
0 \\
0
\end{array}\right) \text { or }\left(\begin{array}{l}
0 \\
1 \\
0
\end{array}\right) \\
\text { and } m_{i j}=\frac{1}{\rho}\left(\begin{array}{ccc}
L & \cdot & \cdot \\
\cdot & L & \cdot \\
\cdot & \cdot & C
\end{array}\right), & n=\left(\begin{array}{l}
0 \\
0 \\
1
\end{array}\right),
\end{array}
$$

so that we get three distinct eigenvalues for wave propagation in the plane perpendicular to the symmetry axis (or any other direction, except for waves propagating parallel to the symmetry axis, which only have two eigenvalues). See Figure 3.1 for illustration of the distinct velocities. In the case of a propagation direction perpendicular to the symmetry axis, the three wave-speeds are

$$
P_{1}=\sqrt{\frac{A}{\rho}}, S_{1}=\sqrt{\frac{N}{\rho}}, \text { and } S_{2}=\sqrt{\frac{L}{\rho}},
$$

while the two wave-speeds for propagation parallel to the symmetry axis are

$$
P_{2}=\sqrt{\frac{C}{\rho}} \text { and } S_{2}=\sqrt{\frac{L}{\rho}},
$$

where $A>N>L$, for anisotropy caused by alternating layers or vertical aligned microcracks. For a layered medium, a P-wave that is polarised across the layers is 

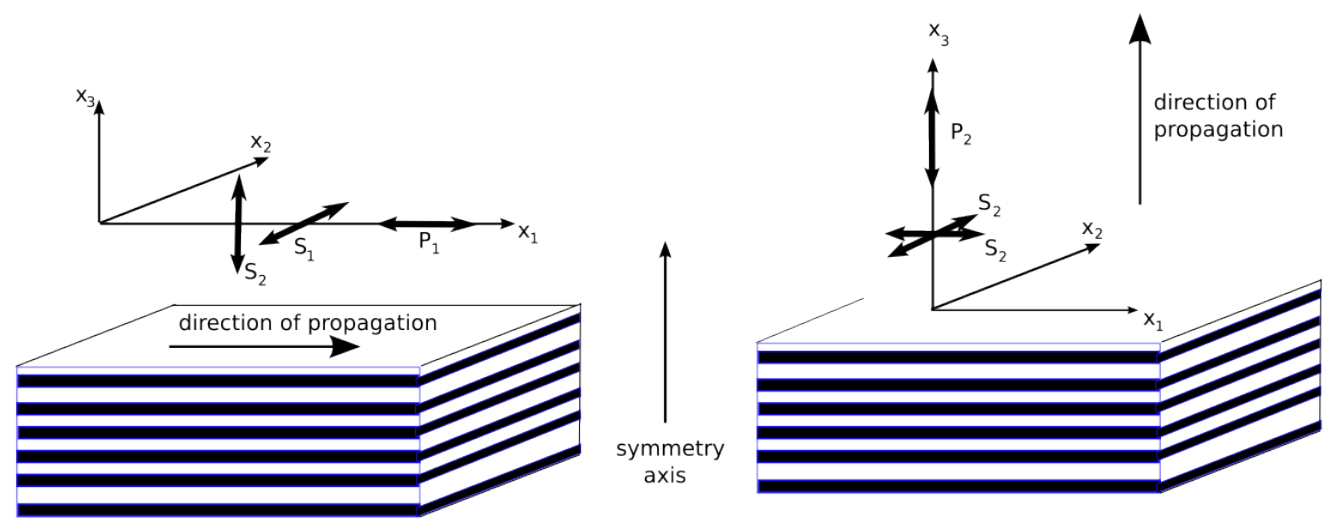

Figure 3.1.: Effect of transverse isotropy on wave velocities.

Left: Propagation perpendicular to the symmetry axis. Shear wave speed differs for waves oscillating across layers resp. within the planes. In this case the S-wave splits into SH and SV.

Right: No shear wave splitting, but the $\mathrm{P}$-wave is slower in this direction than it is for propagation within the planes. The speed of the shear wave is also slow $\left(\mathrm{S}_{2}\right)$, even though the polarisation is the same as for a wave with fast speed $\left(\mathrm{S}_{1}\right)$ in the case of horizontal propagation. (After Babuška and Cara, 1991).

generally slower than one that moves the particles along the layers. This can be shown mathematically (Backus, 1962), but also follows intuition. P-wave velocity changes due to anisotropy are hard to measure, whereas the contrast between slow and fast shear wave velocities can easily be measured. The whole concept of an anisotropic layered medium can be seen as an analogue to a medium with cracks (recall Section 2.2.3). But shear wave propagation does not follow intuition in the case of a layered medium.

A shear wave polarised along the layers travels with the faster wave speed $\left(\mathrm{S}_{1}\right)$ in the case of horizontal propagation, but with slower wave speed $\left(\mathrm{S}_{2}\right)$ when propagating vertically (see Figures 2.1 and 3.1).

If the fast orientation of shear waves in a medium with microcrack-induced anisotropy is obtained with shear wave splitting measurements, conclusions about 


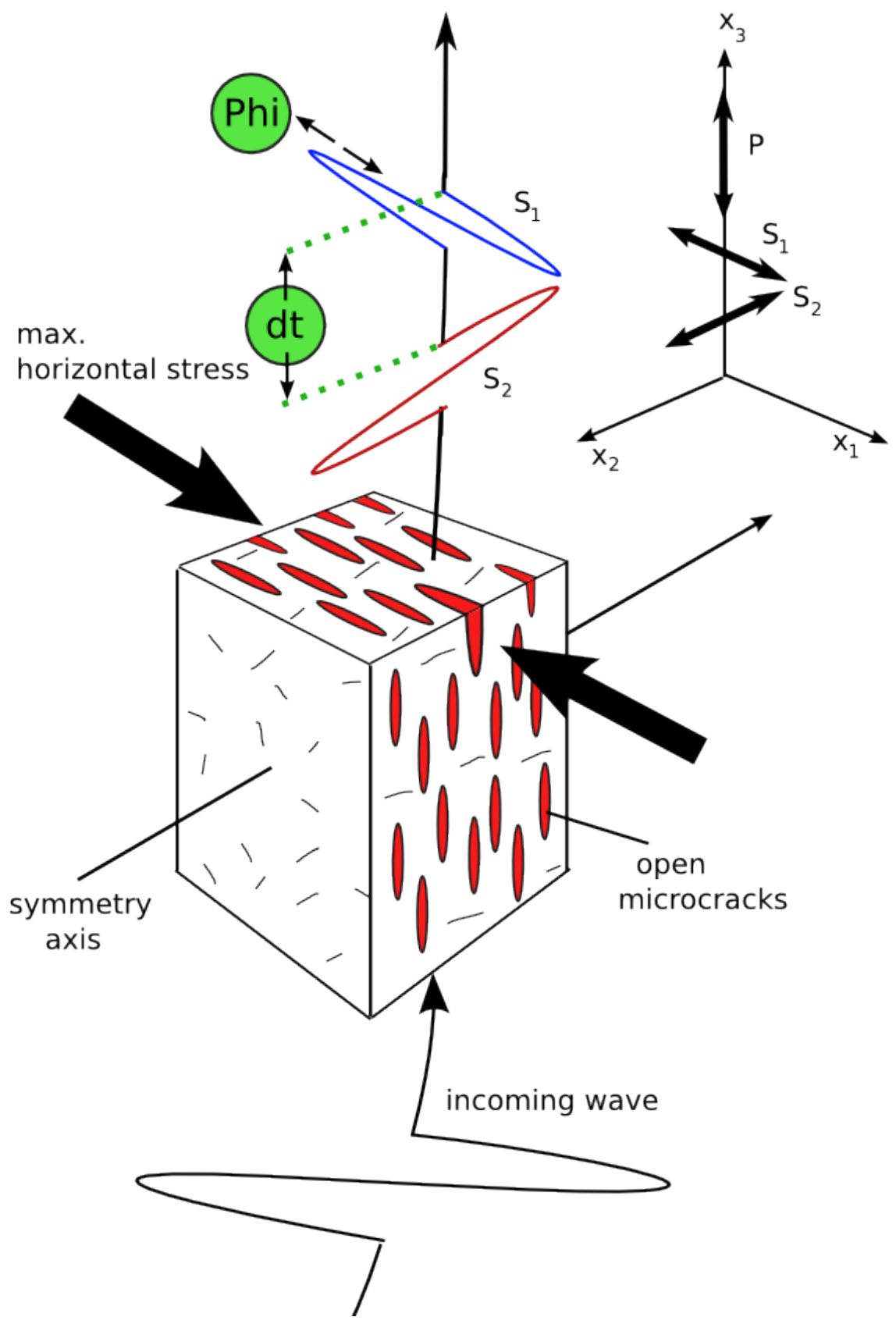

Figure 3.2.: Crack-induced shear wave splitting. An S-wave is decomposed into a slow and a fast component and the polarisation directions reveal the symmetry of the cracked medium and the direction of the maximum horizontal stress. The splitting of the shear waves can be quantified by the splitting parameters Phi $(\Phi$, polarisation direction of the fast shear wave, positive angles are usually measured clockwise from $\mathrm{N}$ ) and dt (delay time $\delta t$ between fast and slow shear wave, see text for details). Cracks that are not aligned parallel to the maximum stress tend to close, which leads to anisotropy with fast orientation parallel to the orientation of maximum horizontal stress. Width of crack-opening is exaggerated. 
crack alignment and thus the state of stress can be drawn. Results reflect an average stress state along the ray path (Gerst and Savage, 2004).

Figure 3.2 illustrates the phenomenon of shear wave splitting. An arbitrarily polarised S-wave enters the anisotropic medium (in this case the anisotropy is induced by aligned microcracks) and splits into two wavelets, which are polarised in fast and slow orientation, approximately orthogonal. This can be visualised as the decomposition of a polarisation vector.

While the polarisation of an S-wave travelling through an isotropic medium depends on the source, the polarisations after travelling through anisotropic media are controlled by the properties of the medium and its fast and slow direction, which vary with propagation direction (Thomsen, 2002). If the split waves travel through another layer with different fast and slow orientation, they will each split again, now reflecting the orientations of the last pervaded medium. But even with the presence of multiple layers, information about the deeper layers can be retrieved from shear wave splitting (Silver and Savage, 1994).

Shear wave splitting is quantified by two parameters (see Figure 3.2):

$\Phi$ : The polarisation angle of the fast wave.

$\delta t$ : The delay time between fast and slow wave,

where

$$
\delta t=L\left(\frac{1}{S_{1}}-\frac{1}{S_{2}}\right)
$$

with $\mathrm{L}$ as the path length through the anisotropic medium, and $S_{1}$ and $S_{2}$ are the fast and slow shear wave velocity respectively (Savage, 1999). 


\subsubsection{How to measure shear wave splitting}

A detailed tutorial on how to measure shear wave splitting is given by Savage (1999). For this study seismic anisotropy has been measured with the shear wave splitting method and all such measurements were based on the algorithm of Silver and Chan (1991), hereafter referred to as SC91. It searches for a best-fitting pair of splitting parameters ( $\Phi$ and $\delta t)$ by performing a grid-search over a range of their possible values. This method is explained in detail in Section 4.3.1, p. 76 .

Some other algorithms to measure the shear wave splitting parameters are briefly presented in Section 3.2.1. Fouch and Rondenay (2006) give an overview of the SC91 algorithm and other shear wave splitting methods. Crampin and Gao (2006) group shear wave splitting techniques into 1) display (manual), 2) semi-automatic and 3) automatic and point out many complications and fallacies (see also Section 3.2.2).

\section{Spatial resolution}

The Fresnel zone of the phase - the ray-perpendicular section of the ellipsoid of revolution (in homogeneous media) that affects the wave field, following Huygens' principle (Kravtsov and Orlov, 1980) - determines the lateral resolution for shear wave splitting measurements (e.g. Fouch and Rondenay, 2006). Lateral resolution is better than vertical resolution, because the ray paths sample a well defined region laterally, but measure integrated effects along the ray path vertically. For a spherical anisotropic inclusion, Chevrot et al. (2004) find $a_{\text {crit }}=0.75 \sqrt{\lambda z}$ as a critical radius, where $\lambda$ is the wavelength and $z$ is the depth of the inclusion. For smaller objects (or anisotropy variations within smaller length scales), the relation between subsurface anisotropy and measurements on the surface is not straightforward and simple geometrical ray theory is invalid (Chevrot et al., 2004).

At a geothermal site, Elkibbi et al. (2005) estimate the area that is sampled laterally with shear wave splitting measurements as 500 m. Similarly, Bianco et al. (2006) 
report that shear waves sample only small lateral portions at Mt. Etna. The good lateral resolution is helpful for monitoring temporal changes at volcanoes or production sites, as for such scenarios the anisotropy is expected to change within a certain lateral area, which can be mapped with splitting measurements at several stations.

For local earthquakes at Mt. Ruapehu, Gerst (2003) evaluates typical Fresnel zone radii as $300 \mathrm{~m}$. With the formula of Chevrot et al., this yields a critical radius for an inclusion of $450 \mathrm{~m}$ to be clearly detected with shear wave splitting measurements.

\section{The shear wave window}

A cone of all the incident angles which are less than a critical angle $i_{c}$ to the vertical is called the shear wave window (Nuttli, 1961; Booth and Crampin, 1985). If a shear wave is incident at an angle larger than the critical angle, S-to-P reflections at the surface disturb the seismic record (Nuttli, 1961) and shear wave splitting measurements give erroneous results. The critical angle is calculated from wave velocities:

$$
i_{c}=\arcsin \left(v_{S} / v_{P}\right) \approx 35
$$

if $v_{P} / v_{S} \approx \sqrt{3}$ (Nuttli, 1961). The shear wave window accounts for surface effects, so the angle of incidence is measured to a line perpendicular to the surface at the station site. Deviations from a standard shear wave window cone around a strictly vertical line are possible through topography, and a local slope correction can be necessary. Following Snell's law (Snellius, 1617), a steeper angle of incidence is caused when a seismic wave has to pass low velocity material. Because of this, low velocity material near the surface - which is common at volcanic areas - can effectively broaden the shear wave window (Gerst and Savage, 2004). 


\section{Algorithms to measure shear wave splitting}

There are several algorithms to perform shear wave splitting measurements and retrieve the shear wave splitting parameters, the polarisation orientation of the fast shear wave $(\Phi)$ and the time delay between fast and slow shear wave $(\delta t)$. Singlephase methods, such as Silver and Chan (1991) handle each event on each recording station separately, while a group inversion algorithm analyses whole wave fields instead of single phases (Menke and Levin, 2003).

A comparison of two classical single-phase methods and a group inversion approach is given by the modelling studies of Levin et al. (2007). Their analysis of synthetic data points out a critical deficiency of the single-phase methods: the detection of null measurements (splitting measurements on unsplit shear waves; see Section 3.2.2). These authors suggest to employ different methods for comparison, as the singlephase methods diverged in the presence of complex anisotropy. They state that the single-phase methods gave satisfactory results only for a single and simple anisotropic body. However, Silver and Savage (1994) and Özalaybey and Savage (1994) found and analysed two distinct layers of anisotropy in their shear wave splitting studies using single-phase measurements, which would be special cases in multiple-phase studies.

\subsubsection{Complications for shear wave splitting measurements}

Shear wave splitting allows simple and straightforward measurement of subsurface anisotropy. However, there can be complications that need to be considered while interpreting the measurements. Shear wave splitting parameters represent a pathintegrated effect and the vertical resolution is limited (Savage, 1999). This makes it difficult to distinguish depths and layers, and assumptions are often necessary. It can be useful to look for a difference in anisotropy for events of different source depths (Savage, 1999, and references therein). Fouch and Rondenay (2006) estimate 
a minimum recording time of one year for a seismic station to achieve a sufficient backazimuthal variation for studies of teleseismic events. For local studies, events with much lower magnitudes can be recorded, so that shorter temporary deployment can be sufficient. For this study of anisotropy at Mt. Ruapehu in New Zealand, the backazimuthal coverage was fairly good, as sources came from all directions (see Figure 5.3, p. 96).

A summary of reasons for the difficulty of shear wave splitting measurements is given by Crampin and Gao (2006). They name impedance effects (attenuation and different frequencies in fast and slow shear wave, which can result in different waveforms for the fast and slow split wave), non-orthogonality (polarisations are not exactly orthogonal), noise effects (conventional noise and P-coda), effects of surface and sub-surface topography, and scattering ( $90^{\circ}$-flips and other effects). Some complications that have to be considered for shear wave splitting measurements are described below.

\section{$90^{\circ}$-flips}

While changes in polarisation direction could colloquially be denoted as flips, this text will follow the notation of the APE-theory, where the term $90^{\circ}$-flip is used to describe a certain phenomenon, but not changes in polarisation direction in general. $90^{\circ}$-flips occur when the pore-fluid pressure is very high and cause a $90^{\circ}$ change in fast orientation $\Phi$ without a change in stress orientation (Crampin and Chastin, 2003). This has been observed at a hydrocarbon reservoir injection experiment (Angerer et al., 2002).

But $90^{\circ}$-flips are at most a local phenomenon, as they are restricted to high pressure zones (e.g. above active faults; Crampin and Chastin, 2003) and are caused by high fluid-pressure, which is at least unlikely for larger regions (Gerst and Savage, 2004). 

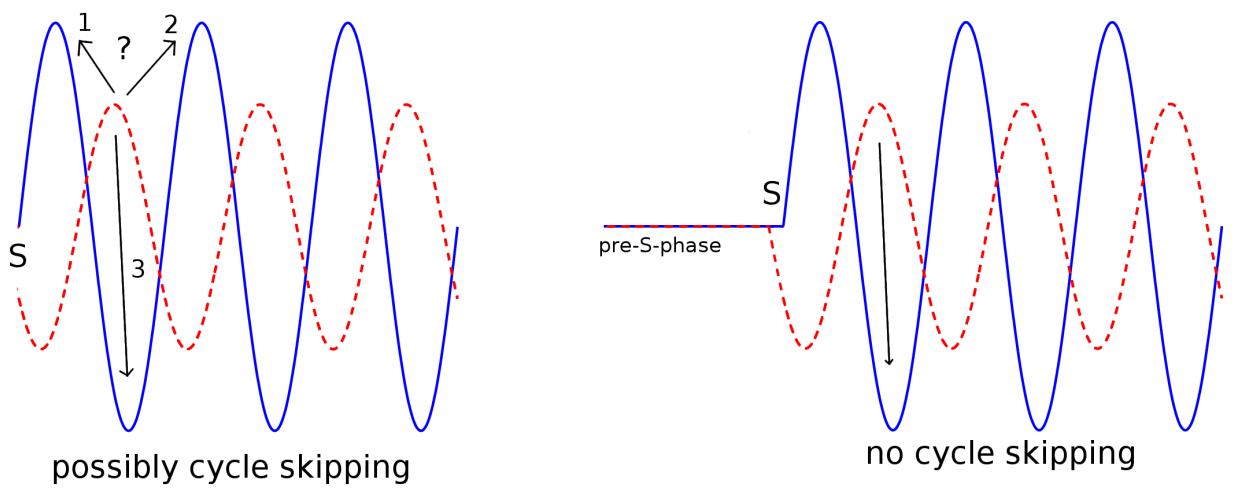

Figure 3.3.: Cycle skipping and choice of measurement window. If the measurement window (Section 4.3.2) only contains a sinusoidal waveform, the measurement can suffer from cycle skipping, because the waveforms can be matched in several ways (left, arrows 1-3). The uncertainty of the delay time is a multiple of half a period length. If parts of the seismogram before the S-wave arrival are included, the waveforms can only be matched in one way (right). It becomes also obvious that the dashed line represents the "true fast" wave in this example, leading the slow wave (solid line).

\section{Cycle skipping}

Cycle skipping is a type of error in shear wave splitting measurements, where waveforms are not correctly matched ("a cycle is skipped"; see Figure 3.3). It occurs especially when the frequency band of the data is narrow and the signal appears sinusoidal. To prevent cycle skipping, parts of the seismogram before the S-phase arrival are included in the splitting analysis (see Section 4.4.1 for details). Cycle skipping not only leads to false delay times, but can also introduce a $90^{\circ}$-error of the fast orientation, if the slow wave is misinterpreted as the fast wave (arrow 1 in Figure $3.3)$.

\section{Null measurements}

If an incoming S-wave is (coincidentally) polarised in the fast or slow orientation of the anisotropic medium, no shear wave splitting will occur. Only one S-wave will propagate, with the velocity of the fast wave for a wave originally polarised in the fast orientation, or with the velocity of the slow wave in the case of polarisation in 
the slow orientation (Figure 3.4). An unsplit shear wave typically shows no or only minor energy on the transverse component (Figure 3.5).
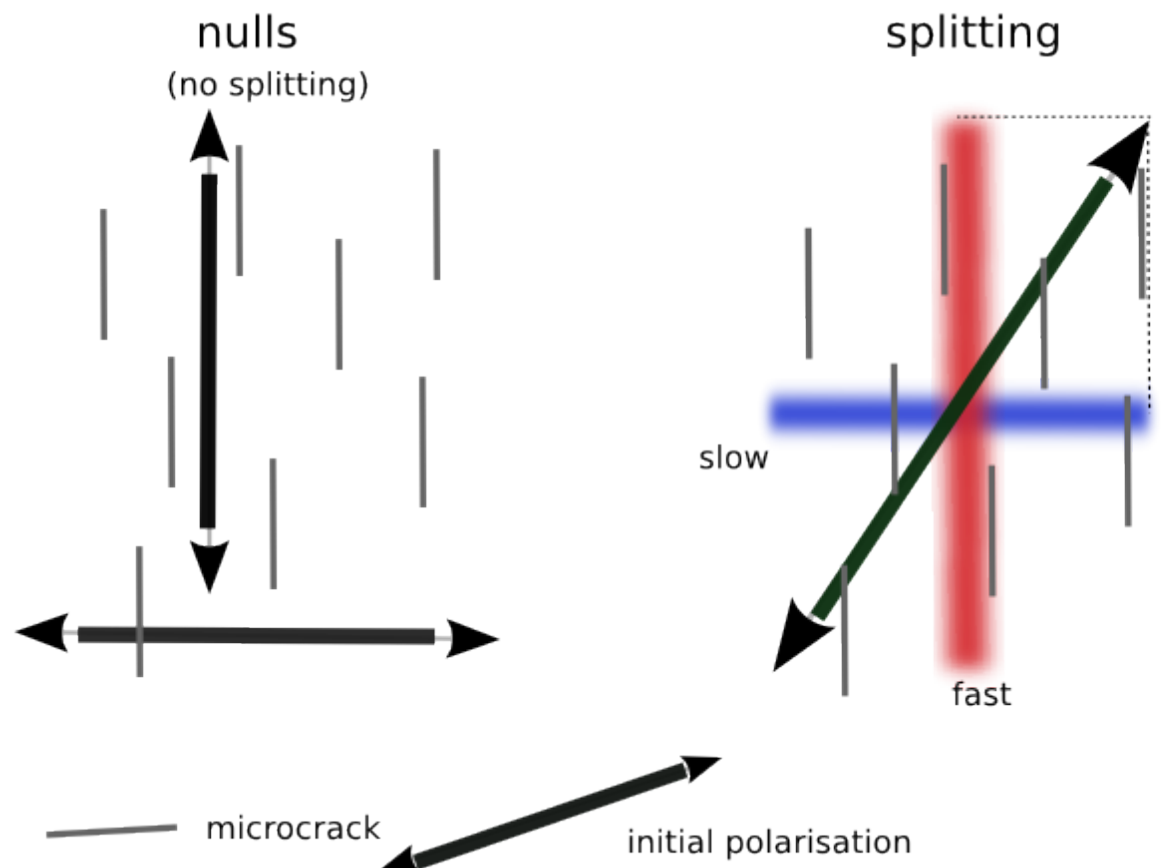

Figure 3.4.:

Left: Initial polarisation of the S-wave in the fast (or slow) orientation of the medium leads to no splitting of the shear wave. Only one shear wave propagates with the fast (or slow) wave speed and only one shear wave arrival is visible on the seismogram. For shear wave splitting measurements, this is called a null measurement.

Right: Oblique angle between initial polarisation and fast orientation. The shear wave is split into a fast (red bar) and a slow component (blue bar). The polarisations of these two components are perpendicular to each other an travel independently with different speeds.

In such a case, some shear wave splitting measurement algorithms tend to give erroneous results. Instead of giving an expected null measurement with wave propagation perpendicular to or along the fast orientation, single-phase algorithms sometimes output large delay times and have a $90^{\circ}$ ambiguity for the fast orientation (see Figure 4.10, p. 86 for an example diagnostic plot of a null measurement). To suppress this unwanted effect, it might be necessary to interpret measurements as null if the time 


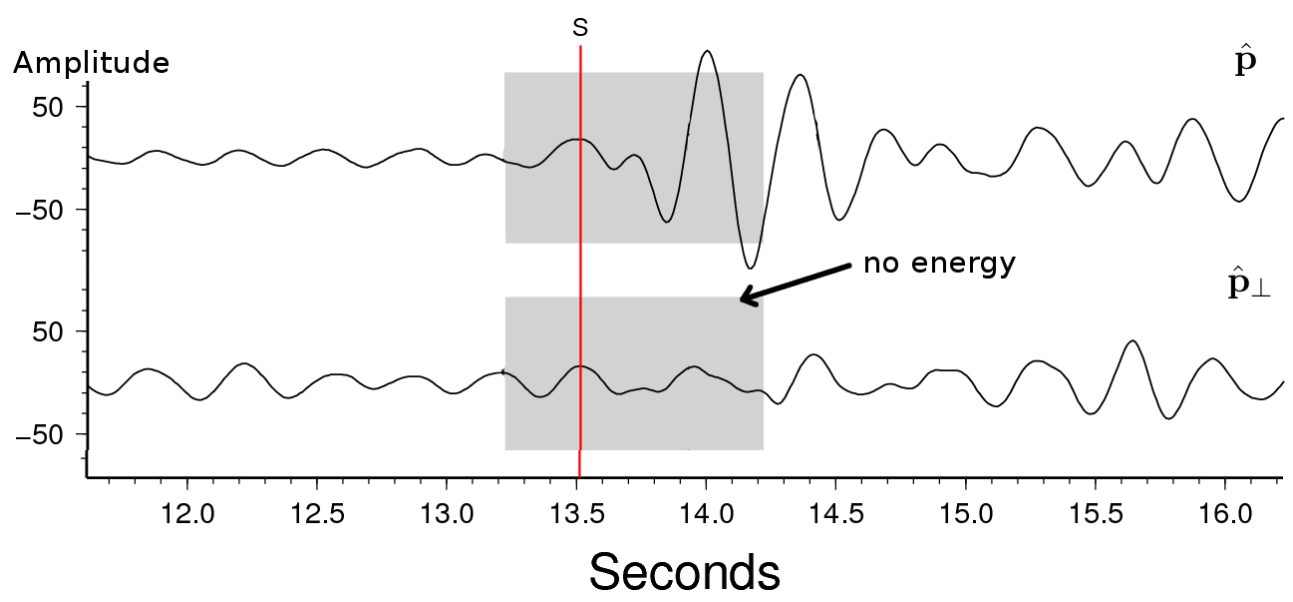

Figure 3.5.: Null measurement: a lack of energy on the component perpendicular to the initial polarisation direction (lower trace) indicates the absence of any detectable shear wave splitting for this event (2005.002.TUVZ.2346225 filtered with $2-3 \mathrm{~Hz}$ bandpass filter). The vertical red line marks the Sphase arrival time, the grey box shows the measurement window (see Section 4.3.1).

delay is close to the allowed maximum (Levin et al., 2007). Hence the definition and detection of null measurements turns out to be critically important.

For a detailed discussion of null measurements see Silver and Chan (1991) and Wüstefeld and Bokelmann (2007). A description of how the null measurements problem was addressed for the development of an automatic measurement tool in this study, see Section 4.4 .2 (p. 86).

\section{Geometrical complications}

In a recent study, Levin et al. (2007) investigated the influence of observational geometry on the results of shear wave splitting measurements. Discrepancies between expected and observed fast propagation directions at subduction zones can occur at a mantle wedge and are commonly observed.*

In the case of a complex geometrical structure of the anisotropic region, e.g. in a subduction regime with an anisotropic mantle wedge, the results are found to be

\footnotetext{
${ }^{*}$ Levin et al. use long-period waves $(5 \mathrm{~s})$ for their modelling.
} 
dependent on the algorithm. It confirms the findings of Menke and Levin (2003), who report algorithm-dependent values, if shear wave splitting is applied to regions with complicated splitting (which could be caused by a complex geometry of the anisotropic region).

With a variety of source-receiver geometries and a broad backazimuthal distribution of the sources, the single-phase methods can still provide useful information, as long as the results are interpreted with caution and awareness of the limitations. In the simple case of a uniformly anisotropic single layer the tested methods obtain mainly good results.

\section{Distinguishing heterogeneous anisotropy}

Seismological measurements can suffer from path effects, especially when apparent temporal changes occur for events from different locations (Section 2.3). The effect of heterogeneity on the waveform is not dependent on polarisation of the shear wave, while the effect of anisotropy is. Plotting single measurements on a map view (fast orientation $\Phi$ for each earthquake-station pair) can reveal lateral variations in anisotropy (Savage, 1999). Good azimuthal coverage and dense station spacing are needed to be able to make non-ambiguous interpretations.

Shear wave splitting parameters reflect the path-integrated effect of anisotropy along the ray path. It is not trivial to detect where (at what depth; mantle or crust) the anisotropy occurs (Fouch and Rondenay, 2006). If applicable, interpretation of shear wave splitting measurements can be leveraged by the integration of other geophysical data, such as electrical anisotropy or gravity data (Fouch and Rondenay, 2006). 


\subsubsection{Other methods}

Shear wave splitting is not the only effect of seismic anisotropy on the wave field and there are other methods to measure the anisotropy of a medium. It can be measured with receiver function methods (Bostock, 1998), P-wave delay times (Hess, 1964; Babuška et al., 1984), or Love- and Rayleigh waves (Anderson, 1961; Tanimoto and Anderson, 1985). A detailed discussion of other approaches and a comparison of techniques has been published elsewhere (Fouch and Rondenay, 2006, and the many references therein).

Compared to the shear wave splitting method, measuring seismic anisotropy with surface waves yields a better vertical resolution, while the lateral resolution is poor (Forsyth, 1975; Forsyth and Li, 2005). For the monitoring of temporal changes of crack-induced anisotropy in the vicinity of volcanoes, the shear wave splitting method seems to be the most suitable, in particular because fluid-filled microcracks have a larger effect on shear waves than on other wave-types (Crampin et al., 1984).

\subsection{Automatic splitting measurements}

With increasing deployment times and addition of new permanent stations the amount of available data worldwide is continuously increasing (e.g. from the USArray, a network of 400 three-component seismometers; Busby et al., 2005). The more data that are available, the more time consuming manual measurements will become. This calls for the development of automatic measurement techniques that minimise the human workload for splitting measurements. Manual methods can also be biased, whereas automatic measurements promise objective and reproducible results.

Algorithms like SC91 (Section 4.3.1) usually require an S-phase arrival time and a measurement window to be selected. Before the measurement it might be necessary to apply a certain filter to improve the signal to noise ratio. Events which suffer 
from a high noise level, an emergent onset of S-wave energy instead of a desired impulsive onset, or an overlap of $\mathrm{P}$ - and S-wave coda, have to be rejected. After the measurements has been performed, several quality control steps are employed to include only reliable measurements into further interpretation.

For measuring anisotropy in the upper mantle, Evans et al. (2006) developed a method for automatic SKS measurements based on the SC91 algorithm. They processed 20,000 seismograms and found only 2 per cent of the data produce reliable measurements when using their automatic method. The rejection of large portions of the data seems to be a general downside for automatic methods. Strict requirements of data quality lead to the rejection of data that would probably have been usable with a manual method. Automatic methods are therefore only feasible if a large data set is at hand. Even though Evans et al. (2006) focused on SKS phase measurements, some of their approaches and ideas are worth being considered for a study of local phases as well. They found that noisier data tends to result in a time lag $\delta t$ close to the allowed maximum. To identify these cases they allow a large time delay in the first place, but later reject measurements that result in a time lag beyond reasonable values. When comparing their results with earlier manual studies, they found a good agreement for the fast polarisation but not for delay times. This can probably be explained with the scatter that delay times tend to show anyway (e.g. Gerst and Savage, 2004; Crampin and Gao, 2006). The automatic method for SKS splitting measurements is able to reproduce manual results and seems to be reliable. However, satisfying results could only be obtained from stations with at least 7 years recording time (Evans et al., 2006). This and the rejection of $98 \%$ of the data would drastically hinder any identification of temporal changes with this method.

Automation of splitting measurements for crustal events is challenging, as surface topography effects and surface-wave arrivals often disturb the record (Nuttli, 1961; Crampin and Gao, 2006). Peng and Ben-Zion (2004) developed an automatic method 
for shear wave splitting measurements with local earthquakes at the North Anatolian Fault, Turkey. Their method is based on the SC91 alogrithm. They obtained 6,600 usable measurements from 22,000 events in a 6-month data set. These authors used a set of quality criteria to reject erroneous measurements (they tested for stability of results within different measurement windows, signal to noise ratio, waveform match after correction for splitting, minimisation of energy on the component perpendicular to the initial polarisation, standard deviation of the splitting parameters, null measurements and the linearity of the particle motion after correction). They rejected $70 \%$ of their measurements based on these criteria.

The method for automatic shear wave splitting measurements and the quality control criteria used in this study are described in Chapter 4, p. 69.

\subsection{Observations of seismic anisotropy}

There are numerous studies of anisotropy and shear wave splitting. Studies that focus on temporal changes in anisotropy ("anisotropy monitoring"), anisotropy measurements at volcanoes, and anisotropy in New Zealand will be reviewed in this section.

Seismic anisotropy has been observed in the core, the $\mathrm{D}^{\prime \prime}$ layer, the upper mantle, and the earth's crust. Silver (1996) provides an overview of anisotropy in subcontinental mantle, while Savage (1999) reviews shear wave splitting measurements in several tectonic regions. On a global scale, Park and Levin (2002) sum up observations of mantle anisotropy. The fast orientation is generally ridge-normal at the Pacific, Atlantic and Indian ocean ridge, but the connection of flow patterns and anisotropy can be more complicated. For example, the anisotropy distribution is highly heterogeneous in the mantle wedge at the Mariana subduction zone (Pozgay et al., 2007). 
Beneath continents and at subduction zones the outlook is generally complicated, as several factors influence anisotropy, so in this case no "global" generalisation is possible. Also, continental crust itself can have a complex structure with an orogenic history imprinted (Silver and Chan, 1991; Margheriti et al., 1997, 2006).

Fouch and Rondenay (2006) summarise results of studies that measured anisotropy beneath continents (North America, Canada, Australia and southern Africa). These authors also compare several methods to measure anisotropy (shear wave splitting, receiver functions, relative travel times, $P_{n}$ phases, surface waves).

\subsubsection{Crustal anisotropy and its applications}

Crustal anisotropy has been observed in numerous localities. In the lithosphere, anisotropy is more rule than exception, and regions without anisotropy are rare local phenomena (Fouch and Rondenay, 2006). A compilation of measurements of crustal anisotropy and a review of crack-induced shear wave splitting in the crust and its application are given by Crampin (1994) and Crampin and Chastin (2003). Unlike mantle anisotropy, crustal anisotropy that is caused by aligned microcracks (Section 2.2.3) can possibly change in short timescales. However, some skepticism remains about time variations in parameters, because small changes in ray paths may cause large variations in measured quantities.

Crustal anisotropy is typically between SWA $=1.5-4.5 \%$ (Crampin, 1994), but remarkably high values of anisotropy can be observed locally. Środa (2006) obtained values as high as 8-10\% anisotropy in Poland's upper crust. This is explained with the presence of intensely folded metamorphic rocks.

Modelling of crack distributions revealed the existence of a zone with high crack density in The Geysers geothermal fields in California, USA, which has direct implication on possible production of geothermal energy (Elkibbi et al., 2005). The 
splitting measurements show that anisotropy in the reservoir is mainly produced by systems of vertical cracks (Elkibbi and Rial, 2005). Applying an inversion scheme to model the sub-surface crack distribution to the data gives further support to this hypothesis (Yang et al., 2005). Anisotropy has also been measured at several other geothermal fields (e.g. Rabbel and Luschen, 1996; Tang et al., 2005), at borehole experiments (e.g. Bokelmann and Harjes, 2000), hydrocarbon production sites (e.g. Angerer et al., 2002), and in the vicinity of active volcanoes (see below). Many of those applications monitor temporal changes in seismic anisotropy.

\subsubsection{Temporal changes in seismic anisotropy (observations)}

The interpretation of anisotropy and possibly temporal changes in anisotropy remains controversial in many cases. Temporal changes and their use for earthquake prediction were debated for studies in a region in California, USA (Crampin et al., 1990, 1991; Aster et al., 1990, 1991). While Crampin et al. interpreted their observations as temporal changes in anisotropy, Aster et al.-after applying a different analysis method-opposed those claims because of the large scatter in delay times and the absence of any clear trends in the data.

Changes in the source locations after a large earthquake can be mistaken for temporal changes (Peng and Ben-Zion, 2005). In a study of crustal anisotropy before and after two large (M 7.4 and 7.1) earthquakes at the North Anatolian Fault in Turkey, these authors could not find any precursory changes in their data. A study of crustal anisotropy before and after a magnitude 6.0 earthquake at San Andreas Fault, California, also found no temporal variations in anisotropy (Cochran et al., 2006). Munson et al. (1995) found no evidence for temporal changes associated with a magnitude 6.6 earthquake, which occurred within an epicentral distance of less than $6 \mathrm{~km}$ to one of their stations in Hawaii. Precursory phenomena have been observed 
in some studies, but the observability depends on the tectonic setting of the region (Saiga et al., 2003).

There have even been attempts to stress-forecast earthquakes in Iceland (Crampin et al., 1999), but another debate about the quality of the data and the statistical significance of the interpreted temporal changes arose (Seher and Main, 2004; Crampin et al., 2004). It has further been claimed that larger earthquakes could have been forecast, because precursory changes in anisotropy were found in previously unconsidered seismic data (Wu et al., 2006). Temporal changes in anisotropy were observed among several earthquakes (e.g. Saiga et al., 2003; Volti and Crampin, 2003a,b; Del Pezzo et al., 2004). The observability of precursory changes before the 1999 magnitude 7.6 earthquake in Taiwan (Liu et al., 2004; Crampin and Gao, 2005; Liu et al., 2005) was debated - it was not clear if the subtle temporal changes that were pointed out by Crampin and Gao (2005) are statistically significant. Given the sparse data set, and the possible mixture of spatial and temporal changes, Liu et al. $(2004,2005)$ argue that no significant precursory changes occurred. This does not necessarily mean that there have been no changes in anisotropy at all. In the presence of a highly anisotropic shallow crust, small temporal changes can be masked and would hardly be detectable at the surface (Peng and Ben-Zion, 2005). After all, it is not only the question of the presence of temporal changes that needs to be answered, but also the question of the observability of those. Apparent temporal changes in anisotropy must be examined carefully. These considerations were among the main reasons for a station deployment at Mt. Ruapehu in 2002, after data from 1994 an 1998 showed variations in anisotropy (see Section 3.4.5 for details).

Temporal changes of seismic anisotropy have been suggested as a monitoring tool at various environments, especially in the hydrocarbon industry (Crampin and Chastin, 2003). As described in Section 2.2.3, stress-aligned microcracks can change their predominant orientation in very short timescales. This allows the monitoring of 
temporal changes in anisotropy, which are an indicator for a change of the stress field in the medium, and can provide valuable subsurface information for reservoir exploitation (Angerer et al., 2002).

Fluid pressure has been monitored with shear wave splitting measurements at the Krafla geothermal field in Iceland (Tang et al., 2005). It was found that the delay times are especially sensitive to fluid injection (large delay times) or the halting of fluid injection (lower delay times) with a "prompt" response of the crack system.

At the borehole site of the continental deep well drilling program (KTB) in the Bohemian massif in Germany, anisotropy changed significantly within hours after an active fluid injection experiment, presumably due to the influence of induced seismicity on the tectonic stress field (increasing seismicity caused a "stress drop"; Bokelmann and Harjes, 2000). For in-situ stress monitoring, Crampin (2001) developed a cross-borehole stress-monitoring technique that is based on the shear wave splitting method. A similar approach led to the development of an artificial vibration source system. This system has been used to continuously monitor temporal changes in anisotropy near the Nojima fault, Japan, where the Kobe earthquake occurred in 1995. The artificial signals were recorded over 15 months and temporal changes* were recognised at the time of occurrence of two $M>6$ earthquakes (Ikuta and Yamaoka, 2004). The advantage of such a controlled source experiment is that temporal and spatial changes can easily be distinguished, which is not straightforward if natural seismicity is used.

A study of microseismic borehole data supports the hypothesis of anisotropy monitoring being an effective tool to monitor stress changes in the earth's crust (Teanby et al., 2004a). However, these authors could not clearly determine whether ocean

*In this experiment the S-wave travel times were delayed after the earthquakes, but these changes were dependent on propagation direction. 
tides or oil production at the monitored reservoir were mainly responsible for the observed temporal changes.

In a study of the central Apennines, Italy, Piccinini et al. (2006) interpret their observations as temporal changes of anisotropy, possibly induced by a sequence of earthquakes, although spatial variations could not be ruled out completely due to the complex tectonic situation.

\subsubsection{Anisotropy monitoring at active volcanoes}

Temporal changes in anisotropy have been claimed at Mt. Etna in Italy (Bianco et al., 2006), Vatnajökull in Iceland (Volti and Crampin, 2003b), and Mt. Ruapehu in New Zealand (Miller and Savage, 2001; Gerst and Savage, 2004). The limited number of studies probably reflects the drawbacks of such anisotropy monitoring measurements, namely the need of three-component stations, earthquake sources and a fairly dense station network, and the time-consuming and tedious task of performing the measurements. Anisotropy monitoring at volcanoes is a fairly new technique, and further studies at other volcanoes could be promising.

At Mt. Etna, temporal changes in anisotropy were found to be associated with a 2001 eruption (Bianco et al., 2006). A flank eruption ejected lava and was preceded by increasing seismicity. Approximately 70 days before the eruption onset, delay times increased systematically, but decreased shortly (2-3 days) before eruption onset. Polarisation directions changed occasionally (Figure 3.6). Bianco et al. interpret the polarisation changes immediately before the eruption as a realignment of microcrack orientation due to increasing pressure caused by ascending magma. These authors suggest that the observed temporal variations in delay times can be explained by subsurface stress accumulation before the eruption and by stress relaxation shortly before and in the course of the eruption. They normalise the delay time $(\delta t)$ by 


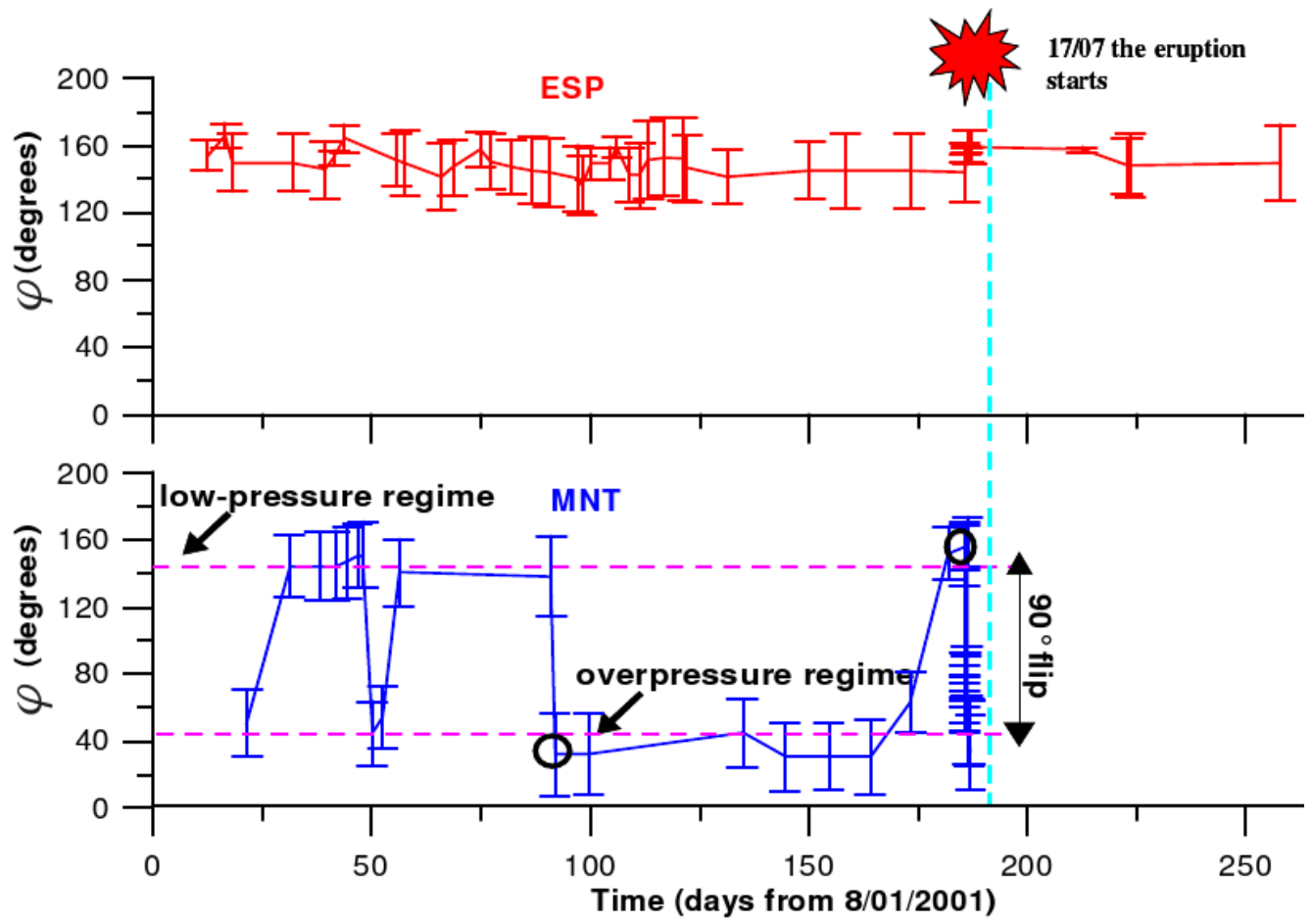

Figure 3.6.: Changes in anisotropy at Mt. Etna (Italy). The orientation of the fast shear wave $(\varphi)$ is plotted against time. The diagram shows nine-point moving averages of $\varphi$ that are plotted every three points (nine-point average in three-event steps). While station ESP (approx. $8 \mathrm{~km}$ from the central crater) shows no obvious variations, station MNT (approx. $4 \mathrm{~km}$ from the central crater) shows several variations with time of about $90^{\circ}$, with a change at day 185 occurring 3 days before the onset of the July 2001 eruptions at Mt. Etna. Annotations are the interpretation of Bianco et al. The dashed horizontal lines show the average values of the two regimes. (Source: Bianco et al., 2006) 
the hypocentral distance and find unusually high values* of up to $20 \mathrm{~ms} \mathrm{~km}^{-1}$ (in agreement with observations in other volcanic regions). An earlier study at Mt. Etna suggests temporal changes of delay times during an eruption in 1989 (Bianco et al., 1998). At Vatnajökull in Iceland, Volti and Crampin (2003b) interpret fluctuations in time delays as temporal changes related to the October 1996 eruption, although the time delay values have a high scatter.

At Mt. Ruapehu, changes of fast polarisation directions were observed after the eruption sequence in 1995/96 (Miller and Savage, 2001; Gerst and Savage, 2004, reviewed in Section 3.4.5).

\subsubsection{Anisotropy in New Zealand and the regional stress field}

The orientation of maximum horizontal stress can be inferred from geological records (volcanic dikes, fault orientations and structural features) and possibly borehole measurements. Faults and vents in the TVZ are mostly found to be aligned NNE-SSW (Nairn et al., 1998; Cole, 1990; Darby and Meertens, 1995) and a NNE-aligned Graben structure has been described (Villamor and Berryman, 2006a). From these observations, the orientation of the maximum horizontal stress $\sigma_{H}$ in the region is estimated as approximately NNE-SSW, with an azimuth of $30-40^{\circ}$. Studies of regional anisotropy find a similar trend (e.g. a trench-parallel fast-direction of $55 \pm 3^{\circ}$ for local events in the TVZ; Audoine et al., 2004). The tectonic configuration for North (Wallace et al., 2004) and South Island (Walcott, 1979) has been described elsewhere.

The causes for anisotropy on both the North and the South Island of New Zealand are discussed by Audoine et al. $(2000,2004)$. Fast polarisations on the lower North Island are typically oriented parallel to the strike of the Hikurangi subduction zone (NE-SW; see Section 1.2.1, Audoine et al. (2000) and references therein). This was observed for local events by Audoine et al. and also agrees with a study of SKS phases

\footnotetext{
${ }^{*}$ Normalised delay times are $8 \mathrm{~ms} \mathrm{~km}^{-1}$ or less for most crustal rocks (Crampin, 1999).
} 
(Marson-Pidgeon et al., 1999) in the same region. Mantle anisotropy can affect deep events and was also reported to have fast orientations aligned NE-SW (Audoine et al., 2004). Crustal anisotropy is quantified as $4 \%$, calculated from delay times.* The average North Island fast polarisation direction was reported as $57^{\circ} \pm 28^{\circ}$, while delay times were found to be scattered (0.1-0.8s; Audoine et al., 2000). At least for shallow events this is not surprising, as delay times are usually scattered in studies of crustal anisotropy (e.g. Crampin and Gao, 2006).

\subsubsection{Results from previous studies at Mt. Ruapehu}

Two previous studies (see below) found temporal changes in seismic anisotropy at Mt. Ruapehu volcano that are likely to be connected with the eruption in 1995/96 (Miller and Savage, 2001; Gerst and Savage, 2004). Another study found some changes in seismic attenuation that support the argument of subsurface temporal changes (Kuehler, 2006).

\section{Miller and Savage: 1994 and 1998 deployment}

This early study found evidence for temporal changes in anisotropy related to the 1995/96 eruption. The data for this study were recorded in two temporary deployments, in January to March 1994 and in February to July 1998. Earthquake data were subdivided into two sets, shallow $(<30 \mathrm{~km})$ and deep $(>50 \mathrm{~km}$, only events with $M_{L} \geq 3$ ). Shear wave splitting measurements were carried out with the SC91 algorithm and revealed temporal changes in the fast orientation for deep events (see Table 3.1 $)^{\dagger}$

*The closest station to Mt. Ruapehu in the Audoine et al. (2000) study was LSTR, $80 \mathrm{~km}$ west of Mt. Ruapehu, which revealed an average fast polarisation direction of $60^{\circ} \pm 27^{\circ}$ for shallow events (in that study: $z<100 \mathrm{~km}$ ).

${ }^{\dagger}$ Note that the data investigated in the Miller and Savage study has been reprocessed with a superior technique (Gerst, 2003). These results are shown in a more illustrative figure in the following section. 


\begin{tabular}{|l|rrrr|}
\hline data subset & $\bar{\Phi}\left[^{\circ}\right]$ & $\pm \bar{\Phi}\left[{ }^{\circ}\right]$ & $\overline{\delta t}[\mathrm{~s}]$ & $\pm \overline{\delta t}[\mathrm{~s}]$ \\
\hline shallow: & & & & \\
1994 & -47 & 7 & 0.10 & 0.01 \\
1998 & -67 & 12 & 0.10 & 0.01 \\
\hline deep: & -36 & 6 & 0.17 & 0.03 \\
1994 & $-110(70)$ & 14 & 0.11 & 0.01 \\
1998 & & & & \\
\hline
\end{tabular}

Table 3.1.: Results from Miller and Savage (2001). Mean values of fast orientation $\Phi$ and delay time $\delta t$ and their standard error are given. Both shallow data sets show NW-SE fast orientation, while the deep events show a change from NW-SE to ENE-WSW. Values for $\Phi$ have been reduced to $-90 \leq \Phi \leq$ 90, except for the orientation for deep 1998 events, where both equivalent azimuths are given, for better comparability with the other values. (As $\Phi$ denotes a circular orientation, there is a $\pi$ ambiguity, as any orientation between 90 and 270 degrees has a corresponding orientation between -90 and 90.)

The NW-SE orientation of the fast polarisation $\Phi$ for 1994 (pre-eruption) data coincides with the direction of maximum compression $\sigma_{H}$ in the Taupo Volcanic Zone (Section 3.4.4). There were two possible interpretations for the observed changes in anisotropy, either a $90^{\circ}$-flip (see Section 3.2.2) or realignment of microcracks due to an overpressured dike. The change of the mean fast orientation between 1994 and 1998 (for deep events and some of the shallow events) was interpreted as the result of a stress release that followed the 1995/96 eruption. The maximum horizontal compression in the TVZ has an azimuth of $30-40^{\circ}$ (see Section 3.4.4) and a magma body is expected to be aligned perpendicular to this direction, because this would have to overcome the least resistance. A magmatic intrusion then induces an overpressure that applies perpendicular to the dike orientation, and adds to the former minimum compression (see Section 2.4). Therefore, in the vicinity of the summit the measurements were said to have been affected by a local pre-eruption stress field. The eruption eventually released the dike overpressure (and microcracks were aligned according to the regional stress field again, or the high pore pressure ceased). The 
fast orientation measured from deep events in 1998 (post-eruption, stress has been released) coincides with the fast orientation at depth and also with the mantle flow orientation derived from regional anisotropy studies (Section 3.4.4).

\section{Gerst and Savage: 2002 deployment}

An additional deployment was carried out in the course of a more recent study. Stations in 2002 were deployed at previous station locations to rule out path effects and substantiate the observation of temporal changes. Data from the 1994 and 1998 deployments were reprocessed with an improved technique. The results are shown in Table 3.2 and Figure 3.8. The criteria for shallow and deep subset were $<35 \mathrm{~km}$ source depth for shallow and $>55 \mathrm{~km}$ source depth for deep events. This has been chosen because of only minor seismicity at a depth of $35-55 \mathrm{~km}$ and contrasting results in these two data sets. For deep events, the fast orientation changed by $80^{\circ}$ between

\begin{tabular}{|l|rrrr|}
\hline data subset & $\bar{\Phi}\left[^{\circ}\right]$ & $\pm \bar{\Phi}\left[^{\circ}\right]$ & $\overline{\delta t}[\mathbf{s}]$ & $\pm \delta t[\mathrm{~s}]$ \\
\hline shallow: & & & & \\
1994 & -28.3 & 3.9 & 0.108 & 0.060 \\
1998 & 13.4 & 5.8 & 0.113 & 0.058 \\
2002 & -30.0 & 2.4 & 0.107 & 0.053 \\
\hline deep: & & & & \\
1994 & -42.8 & 3.6 & 0.231 & 0.129 \\
1998 & 37.4 & 7.5 & 0.118 & 0.063 \\
2002 & 19.2 & 2.7 & 0.272 & 0.175 \\
\hline
\end{tabular}

Table 3.2.: Results from Gerst and Savage (2004). Mean values of fast orientation $\Phi$ and standard error, and delay time $\delta t$ with standard deviation are given. Delay times were not interpreted, because of high scatter and a dependency on frequency, depth and magnitudes.

1994 and 1998, and changed little between 1998 and 2002. For shallow events, it changed $42^{\circ}$ between 1994 and 1998 and back $43^{\circ}$ between 1998 and 2002 (mean values). The 1994-1998 change for shallow events was reversed between 1998 and 2002, so that shallow $\Phi$ values were back to a pre-eruption status. 


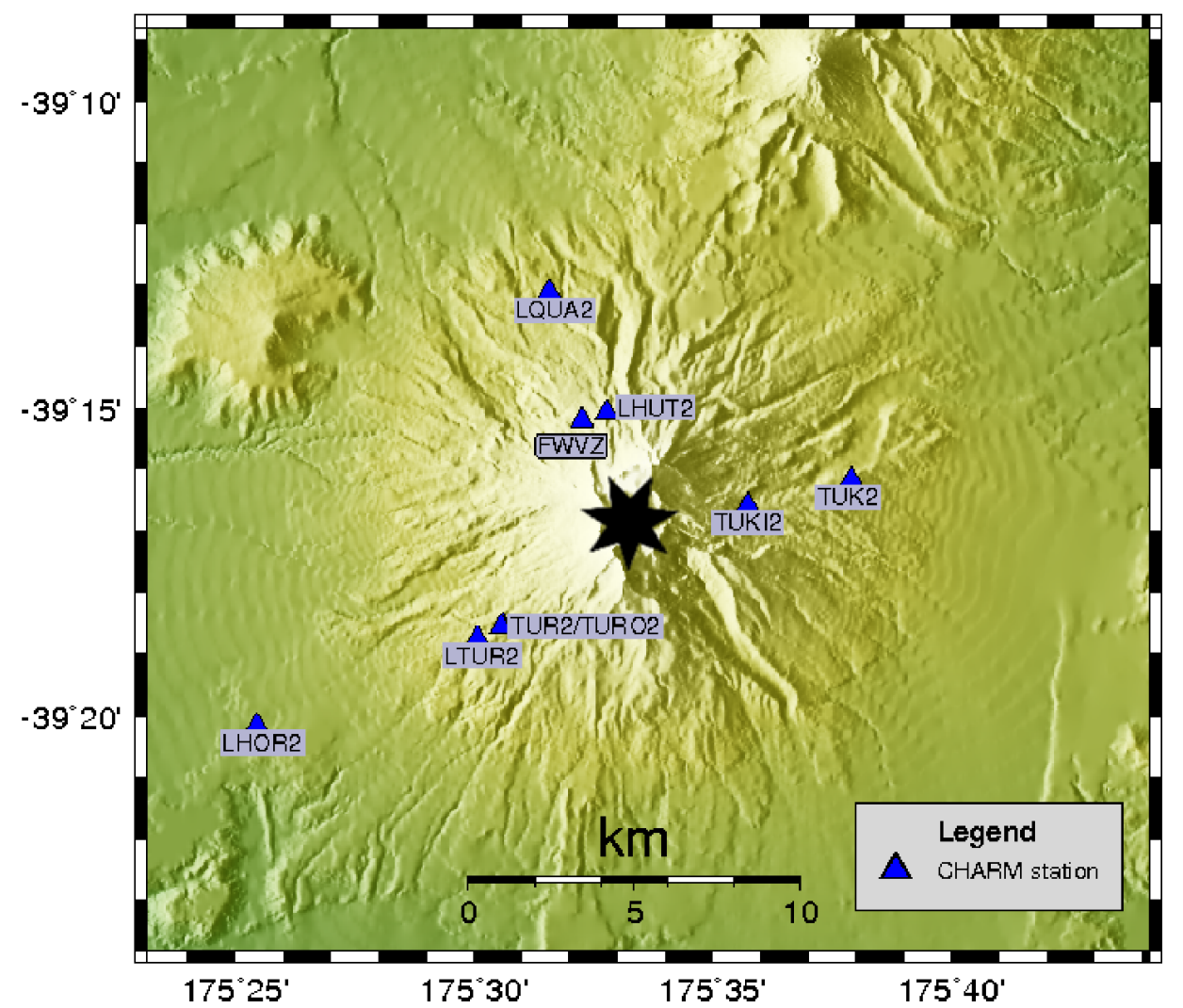

Figure 3.7.: Station network of the 2002 CHARM deployment. The active vent (Crater Lake) is marked with a star. FWVZ is a continuous station of the GeoNet network. Note that TUKI2 was a relocation of TUK2 and TURO2 was a relocation of TUR2. The station locations were chosen after locations from previous deployments in 1994 and 1998.

The temporal changes must have occurred in the crust, because no mechanisms for such rapid changes are known for mantle anisotropy (Gerst and Savage, 2004). These authors conclude that deviations from the constant NNE-SSW fast orientation of mantle anisotropy must be due to an anisotropic region in the crust. Because changes occurred on all stations, anisotropy must have changed in a region of approximately $100 \mathrm{~km}^{2}$. This is explained either with an overpressured dike or with a $90^{\circ}$-flip of the fast orientations. The authors favour the hypothesis of an overpressurised dike, because for $90^{\circ}$-flips, high pore-fluid pressure would have to be present in a large region. An overpressured dike system probably reoriented the stress field and changed the alignment of microcracks prior to the eruption. After a return to regional stress 
1994
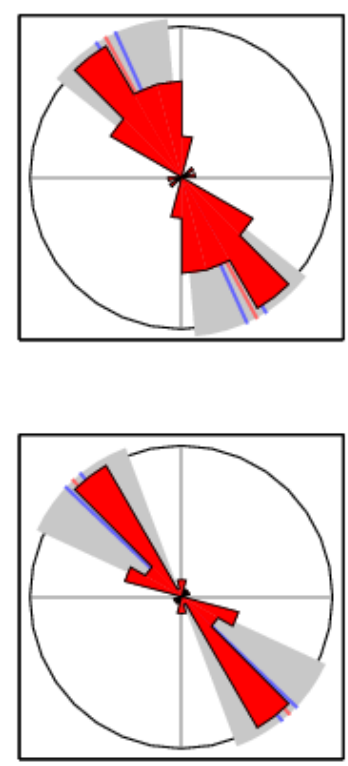

1998

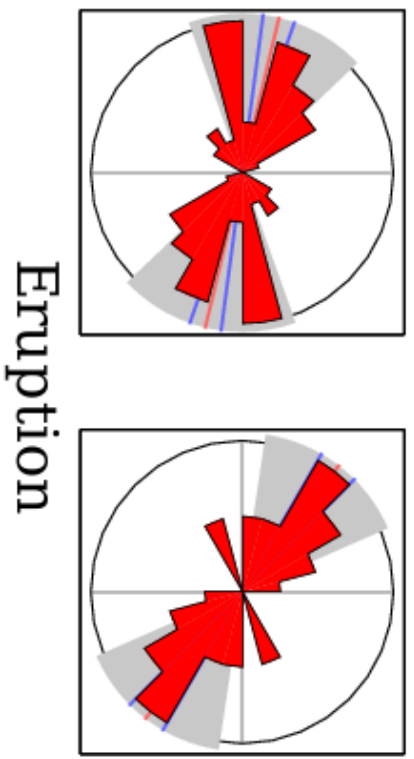

2002

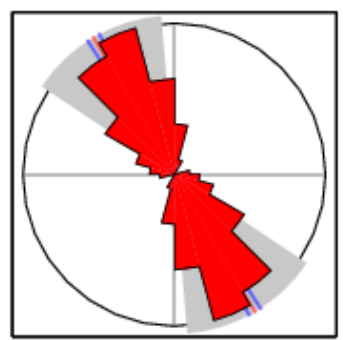

苞

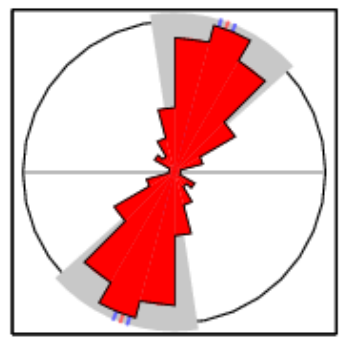

$\frac{7}{\mathbb{0}}$

Figure 3.8.: Results from Gerst (2003), here shown as histograms of the fast direction for 1994, 1998 and 2002 data (deep and shallow events). The grey shade indicates the standard deviation $\left(s_{0}\right)$.

field alignment, a refilling of the dike system could have caused the further changes that were observed in the 2002 data. Differences between deep and shallow events are attributed to the larger wavelengths of the waves from deeper sources, which makes those events less sensitive to changes in crustal anisotropy than shallow events. Delay times were not interpreted in this study, because of high scatter and a dependency on frequency, depth and magnitudes.

It should be noted that Bryan and Sherburn (1999) conclude from seismicity studies that prior to the 1995 eruption sequence the vent structure was open or ductile, hence no stress build-up should have happened until shortly before volcanic activity commenced. But even with an open vent structure there may well have been a stress build-up at depths of 10-15 km (Hurst and McGinty, 1999). 


\section{Method}

This chapter describes the methods that were employed to retrieve and display shear wave splitting parameters and possibly their changes in time. Four key tasks can be distinguished:

- Development of an automatic shear wave splitting technique. Combining and extending the approaches of previous studies on automatic shear wave splitting (see Section 3.3), a methodology for automatic measurement at Mt. Ruapehu was developed. The processing steps of this method are illustrated in Figure 4.1 and a detailed user manual can be found in the Appendix.

- Calibration of the automatic shear wave splitting measurement technique on previously analysed data and comparison of automatic and manual results. This is necessary to test if the automatic technique yields reliable results.

- Application of the automatic technique to new data from 2002 to 2007 to investigate the possibility of temporal changes in seismic anisotropy during that time period.

- Automatic Measurement Tool: Set up an automatic tool that routinely and automatically monitors anisotropy. 


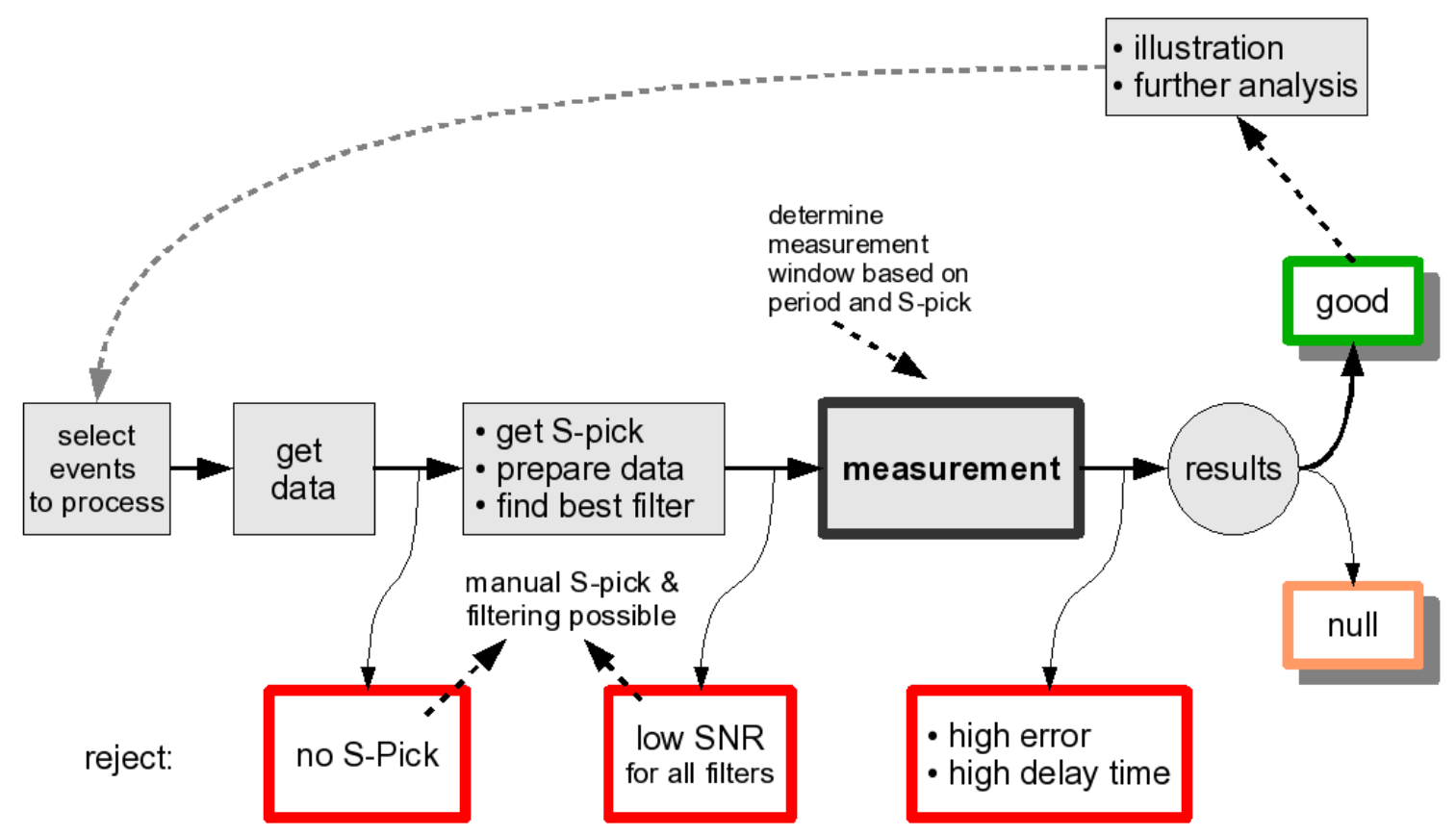

Figure 4.1.: Processing steps for shear wave splitting measurements. All processing steps will be explained in the following sections. A detailed description of the codes is given in Appendix A.

\subsection{Selection and retrieval of data}

Two main data sets, the CHARM temporary deployment data (2002; Gerst, 2003) and data (2003-2007) from permanent GeoNet stations, have been processed. Previously analysed data from 1994 and 1998 was also processed with the new automatic technique for comparison.

Events from the GeoNet network are first chosen by spatial criteria. Following the approach of Gerst (2003), three blocks of earthquakes are selected for further analysis (see Table 4.1). A list of events is created with the Earthquake Search tool on the GeoNet website*. After the events are selected the corresponding data files are retrieved. A script is used to construct the physical address of each file on the

*Website: http://www.geonet.org.nz, Earthquake Search: http://magma.geonet.org. nz/resources/quakesearch/, visited August 10, 2007. Events can be searched for by selecting time and magnitude intervals and an area of interest. A list of events that match the search criteria is generated and can be downloaded. The Earthquake Search tool was only used to retrieve whole years of data. For the automatic monitoring tool (see Section 4.6) this will be done internally within the GeoNet system. 


\begin{tabular}{c|l|r} 
Block & Distance & Magnitude \\
\hline 1 & $\leq 1.5^{\circ}$ & $2.0 \leq x<3.0$ \\
2 & $\leq 3^{\circ}$ & $3.0 \leq x<3.5$ \\
3 & $\leq 5^{\circ}$ & $x \geq 3.5$
\end{tabular}

Table 4.1.: Spatial selection criteria. The distance was measured from the summit of Mt. Ruapehu (Lat. 175.564, Lon. -39.281). Magnitudes are local magnitudes $M_{L}$ (see Section 5.2.1).

GeoNet FTP server and then to download this file. The data are retrieved from the GeoNet system in "Standard for the Exchange of Earthquake Data" (SEED) format. S-phase arrival times from the GeoNet system are requested in a separate file. For a detailed technical description of the programs that have been used for these steps, see Appendix A.

\subsection{Preparation of data}

From the SEED files, SAC (Seismic Analysis Code, see Goldstein and Snoke, 2005) format data files are produced for further analysis. Of those files, only the recordings from stations within a $30 \mathrm{~km}$ radius of Mt. Ruapehu are kept. Magnitude, event depth and hypocentral distance from Mt. Ruapehu are stored in the file header. The files are then moved to a directory according to the station on which they were recorded. A critical incidence angle $i_{c}$ of $35^{\circ}$ is used to reject events that possibly suffer from surface effects (see Section 3.2.1). The incidence angle (from the vertical) of the incoming seismic wave is calculated with the TauP toolkit (Crotwell et al., 1999), using velocities from the ak135 model (Kennett et al., 1995) and a conservative estimate of the near-surface shear wave velocity $(1.6 \mathrm{~km} / \mathrm{s}$; Gerst, 2003).*

For the actual measurement of shear wave splitting, the only requirement for the data is an S-phase pick stored in the data file. S-phase arrival times from the GeoNet

${ }^{*}$ Due to the low near-surface velocity, most events have a sufficiently steep incident angle and pass this criterion. 


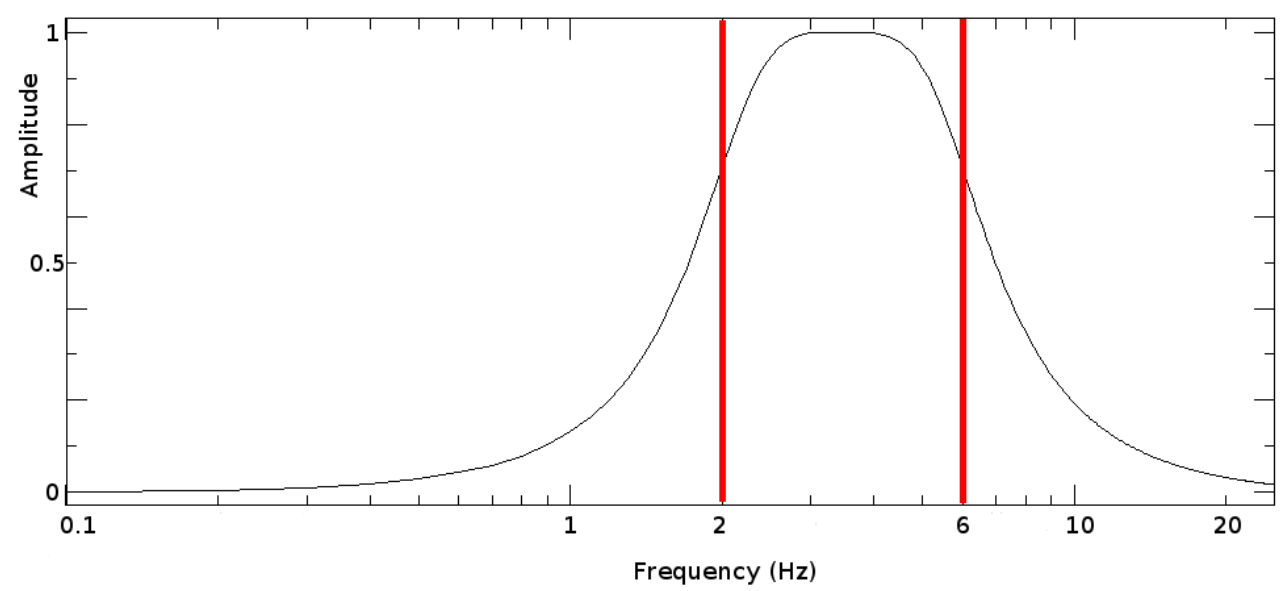

Figure 4.2.: Design of a bandpass filter. The amplitude response curve is shown for a two-pole one-pass Butterworth filter with corner frequencies 2 and $6 \mathrm{~Hz}$ (indicated by the red vertical lines). Frequencies are plotted on a logarithmic scale.

system are written to the files, but the picks can be changed manually for each event if desired. To simplify further handling and processing, event files are cut down to a window of 30 seconds length, ${ }^{*}$ with the S-phase pick at $15 \mathrm{~s}$.

\subsubsection{Application of bandpass filters}

Unlike studies of SKS phases, splitting measurements with crustal events can rarely be done on raw broadband seismic data. The signal to noise ratio (SNR) is too low for most data and phase arrivals can be completely hidden by noise. To remove long-period noise (e.g. lunar tides, ocean waves or wind) and high-frequency noise (e.g. traffic, rain or electrical transformers) a suitable filter has to be applied. For shear wave splitting measurements, the coda of the leading $\mathrm{P}$-wave and other phase arrivals must be considered as noise, too. Since we are interested in the signal of the possibly split shear wave, a bandpass filter is applied to enhance the signal and suppress the noise.

${ }^{*}$ The splitting measurement program allows a maximum of 10,000 data points. If files are cut 15 seconds before and after the S-phase arrival time, this results in files with 3,000 data points for $100 \mathrm{~Hz}$ data, which still contain all relevant information. 


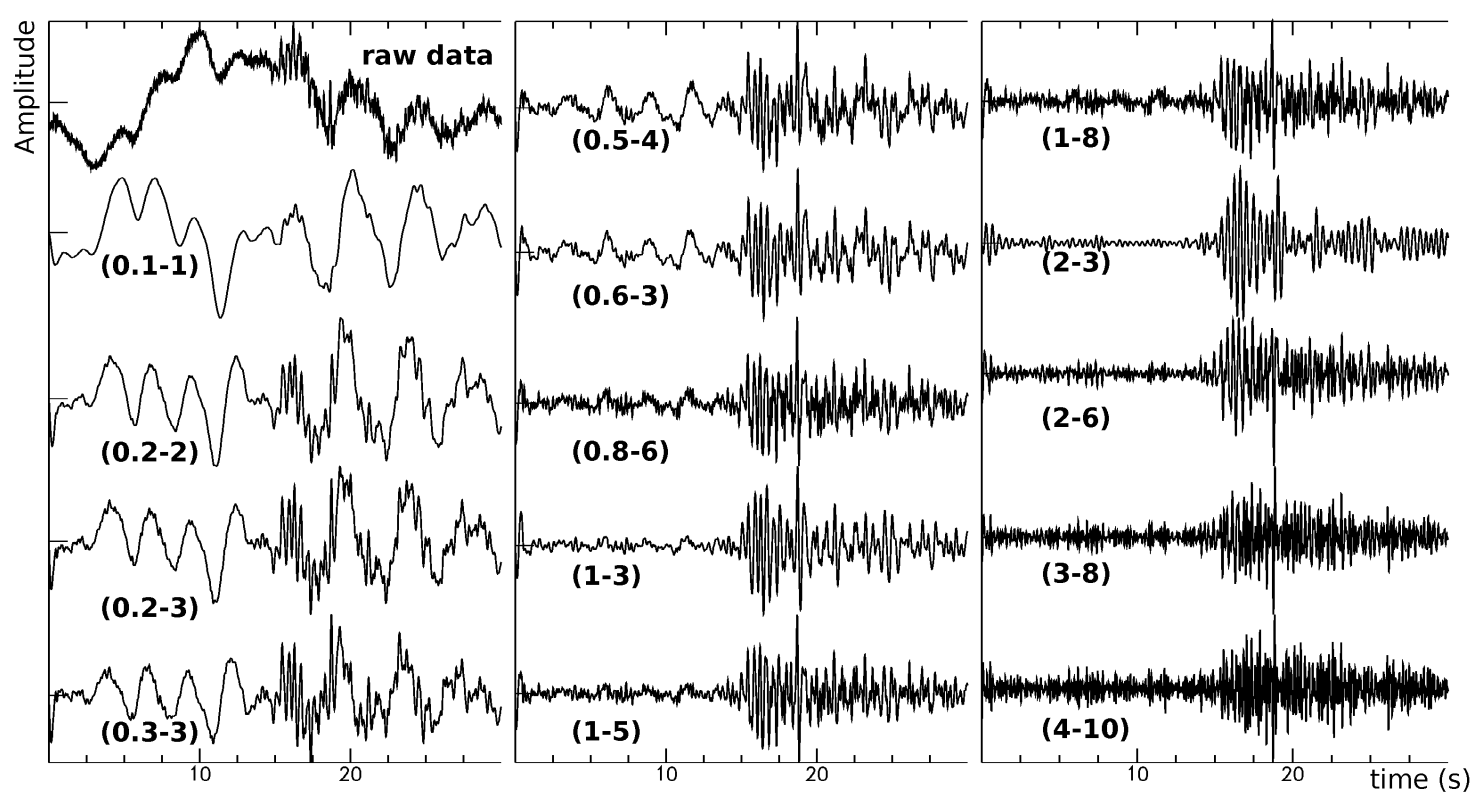

Figure 4.3.: Comparison of all 14 bandpass filters that are used to automatically determine the best filter for any event. The unfiltered seismogram of event 2002.018.01.08.lqua2 is shown in the upper left corner. The filtered seismograms are labelled with the corner frequencies of the applied bandpass filter. Each trace (amplitude vs time) shows a $30 \mathrm{~s}$ window of the east component, with the S-phase arrival at $15 \mathrm{~s}$. With raw data, the actual signal is hardly visible and no good splitting measurement can be done with this data quality. The filters in the leftmost column do not sufficiently remove the long-period noise that is also visible in the raw data and have been automatically rejected by the program. The last two filters (3-8 and 4-10) do not remove high-frequency noise that is visible before the onset of the S-phase. The filter with corner frequencies $2-3 \mathrm{~Hz}$ was automatically chosen for this event. Noise before the phase arrival was mostly removed and the overall quality of the signal was improved (see text for details).

To find the best filter it is possible to compare the waveforms of raw and filtered data and choose the best filter by visual inspection of waveform or amplitude spectrum. The signal of interest is usually at frequencies between 0.2 and $8 \mathrm{~Hz}$ for the analysed local events. For quick processing of a high number of events, I automated this filtering process. Using a SAC macro (findfilt.m; see Appendix A.4.) I apply several different bandpass filters and store all filtered data in separate files. All bandpass filters are two-pole one-pass Butterworth filters (see Figure 4.2 for an amplitude response curve) with lower corner frequencies ranging from $0.1-4 \mathrm{~Hz}$ and higher corner 


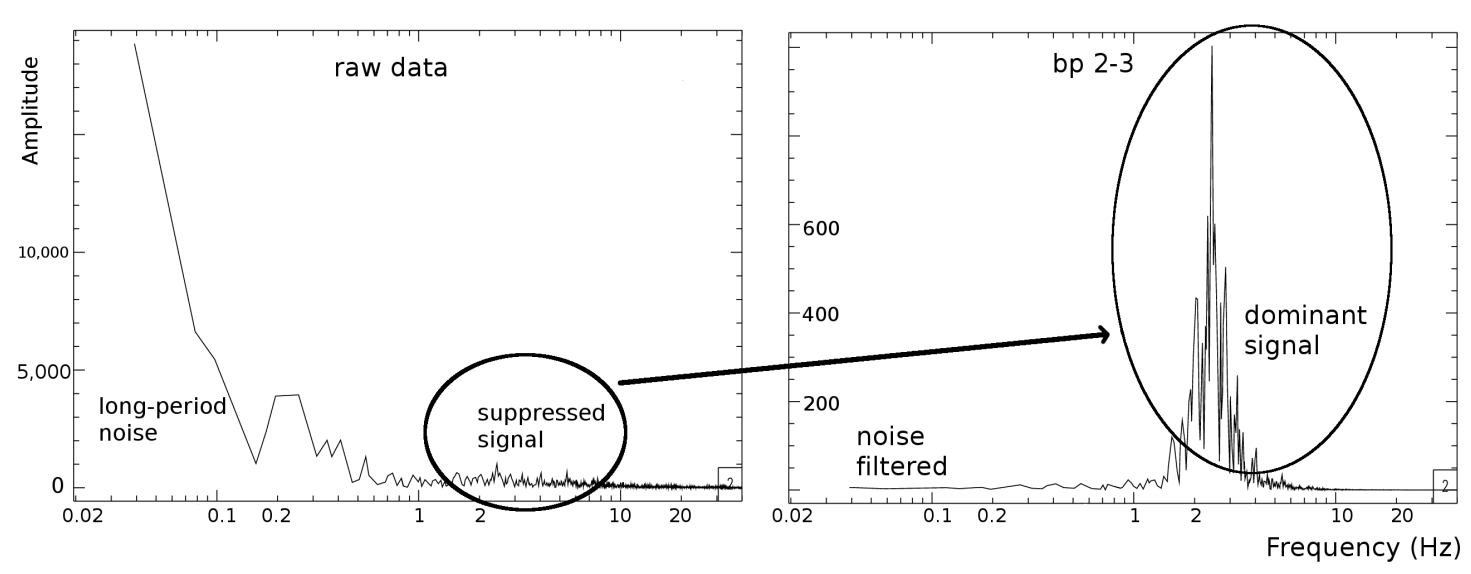

Figure 4.4.: Amplitude spectrum of raw and filtered data. Raw data amplitudes are dominated by low-frequency (long-period) noise below $0.1 \mathrm{~Hz}$. After applying a $2-3 \mathrm{~Hz}$ bandpass filter, the low-frequency noise is removed and frequencies between 1 and $8 \mathrm{~Hz}$ dominate the spectrum. Note the difference in amplitude scales for the two plots. (event 2002.018.01.08.lqua2)

frequencies from $1-10 \mathrm{~Hz}$. The possible corner frequencies of $0.1-1,0.2-2,0.2-3,0.3-$ $3,0.5-4,0.6-3,0.8-6,1-3,1-5,1-8,2-3,2-6,3-8$ and $4-10 \mathrm{~Hz}$ were chosen based on the bandpass filters used in previous studies and from experience. The signal to noise ratio is then calculated for each file (see below) and only up to three high-quality events with the highest ratios are kept for further analysis (see Figures 4.3 and 4.4).

\subsubsection{Signal to Noise Ratio (SNR)}

The signal to noise ratio is calculated from a three second window for each noise and signal. The signal window is selected relative to the arrival of the S-phase and ranges from 0.05 to $3.05 \mathrm{~s}$, while the noise window precedes the S-pick and ranges from -3.05 to $-0.05 \mathrm{~s}$. The small offset of 0.05 seconds has been chosen from experience to account for inaccuracies in the S-picks. The root mean square is calculated for both windows, where

$$
x_{\mathrm{rms}}=\sqrt{\left\langle x^{2}\right\rangle}=\sqrt{\frac{1}{n} \sum_{i=1}^{n} x_{i}^{2}}
$$

is the root mean square for $n$ values $\left\{x_{1}, x_{2}, \ldots, x_{n}\right\}$. A ratio is obtained by dividing RMS(signalwindow) by RMS(noisewindow). This is done for both north and east 


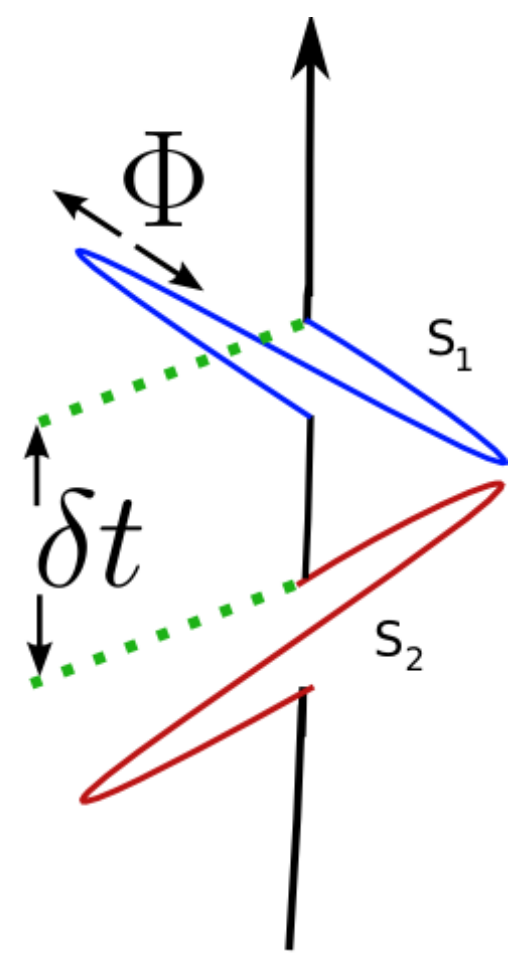

Figure 4.5.: Illustration of the splitting parameters $\Phi$ (usually measured in degree from North, positive angles clockwise) and $\delta t$. $\Phi$ denotes the polarisation orientation of the leading shear wave and has a $180^{\circ}$ ambiguity. $\delta t$ is the delay time between the arrival of the fast and slow shear wave. These two parameters are measured with the Silver \& Chan algorithm.

component and the arithmetic mean of the two ratios is taken as the signal to noise ratio for this event. Only events with $S N R \geq 2$ are considered for further processing, but stricter requirements on data quality have to be satisfied later (see Section 4.4).

\subsection{Shear wave splitting measurement}

There are several techniques to measure shear wave splitting (see Section 3.2.1). In this study, the shear wave splitting parameters ( $\Phi$ and $\delta t$, see Figure 4.5$)$ are retrieved with a new automatic technique, using the algorithm of Silver and Chan (1991) and the cluster analysis method of Teanby et al. (2004b). 


\subsubsection{Silver \& Chan algorithm}

To measure the shear wave splitting parameters, the algorithm of Silver and Chan (1991), hereafter referred to as SC91, is employed. The SC91 method is widespread and has been used in many shear wave splitting studies (e.g. Audoine et al., 2004; Teanby et al., 2004b; Peng and Ben-Zion, 2004; Cochran et al., 2006; Pozgay et al., 2007). This method allows one to measure the splitting parameters by performing a numerical grid-search for an inverse splitting operator that corrects for the splitting. A splitting operator $\Gamma$ (unknown, Equation 4.2) accounts for the splitting, while the inverse operator $\boldsymbol{\Gamma}^{-1}$ reverses the effect of $\boldsymbol{\Gamma}$. If the inverse splitting operator is found, the splitting operator and therefore the splitting parameters are determined. The splitting operator is a function of the splitting parameters:

$$
\Gamma=\exp [i \omega \delta \mathbf{T}(\Phi, \delta t)]
$$

where $\omega$ is the angular frequency, $\delta \mathbf{T}$ is a tensor which depends on delay time and polarisation directions*, and $i$ is the imaginary unit. Applying the splitting operator to an unsplit wave $\mathbf{u}(\omega)$, defined in Equation 4.3, produces a split wave $\mathbf{u}_{s}(\omega)$, as in Equation 4.4:

$$
\begin{aligned}
\mathbf{u}(\omega) & =w(\omega) \exp \left[-i \omega T_{0}\right] \hat{\mathbf{p}} \\
\mathbf{u}_{s}(\omega) & =w(\omega) \exp \left[-i \omega T_{0}\right] \boldsymbol{\Gamma}(\Phi, \delta t) \cdot \hat{\mathbf{p}}
\end{aligned}
$$

where $w(\omega)$ is a wavelet function and $\hat{\mathbf{p}}$ points into the displacement direction and defines the initial polarisation of the shear wave (Rümpker and Silver, 1998). $\mathbf{u}_{s}(\omega)$ describes a split shear wave which can be observed and recorded with a seismometer.

${ }^{*}$ After SC91, where $\delta \mathbf{T}(\Phi, \delta t)=\delta t / 2(\hat{\mathbf{f}} \hat{\mathbf{f}}-\hat{\mathbf{s}} \hat{\mathbf{s}}), \hat{\mathbf{f}}$ and $\hat{\mathbf{s}}$ being the fast and slow polarisation direction, respectively. One would usually speak of polarisation orientation, but $\hat{\mathbf{f}}$ and $\hat{\mathbf{s}}$ in this context are vectors pointing into a certain direction. 
By finding the inverse splitting operator that best turns $\mathbf{u}_{s}(\omega)$ into (or close to) $\mathbf{u}(\omega)$, the anisotropy parameters are retrieved:

$$
\tilde{\mathbf{u}}_{s}(\omega)=\Gamma^{-1} \cdot \mathbf{u}_{s}(\omega)
$$

This is realised by calculating the covariance matrix between two orthogonal components of particle motion in the polarisation plane of the shear wave (radial component):

$$
\mathbf{c}_{\mathbf{i j}}(\Phi, \delta t)=\int_{-\infty}^{\infty} u_{i}(t) u_{j}(t-\delta t) d t \quad i, j=1,2
$$

The eigenvalues of this matrix reflect the presence of anisotropy in the seismogram. If no anisotropy is present (or if - theoretically - the correct splitting parameters are found and the anisotropy is completely removed by correcting the seismogram with the inverse splitting operator), the covariance matrix will have only one non-zero eigenvalue $\left(\lambda_{1}\right)$ with $\hat{\mathbf{p}}$ as eigenvector. If noise is present, the covariance matrix of the split shear wave has still two non-zero eigenvalues $\left(\lambda_{1}\right.$ and $\left.\lambda_{2}\right)$ after the correction. The best splitting parameters are found by searching for the minimum $\lambda_{2}$ to find the most nearly singular covariance matrix.

A graphical representation of the resulting $\lambda_{2}^{\min }$ can be achieved by plotting the particle motion of the fast and slow component before and after the correction (Figure 4.6). The ratio of $\lambda_{1} / \lambda_{2}$ corresponds to the aspect ratio of the particle motion after correction (Silver and Chan, 1991). A minimum $\lambda_{2}$ therefore represents a high aspect ratio of the particle motion, with a linear particle motion as the optimum. The search for $\lambda_{2}^{\min }$ can be illustrated by a contour plot of the errors of the splitting parameters (Figure 4.7). This error surface plot helps to visualise the likelihood of other results for the splitting parameter and shows how well constrained the parameters are for every single measurement.

A split shear wave will show a somewhat elliptical (at least non-linear) particle 

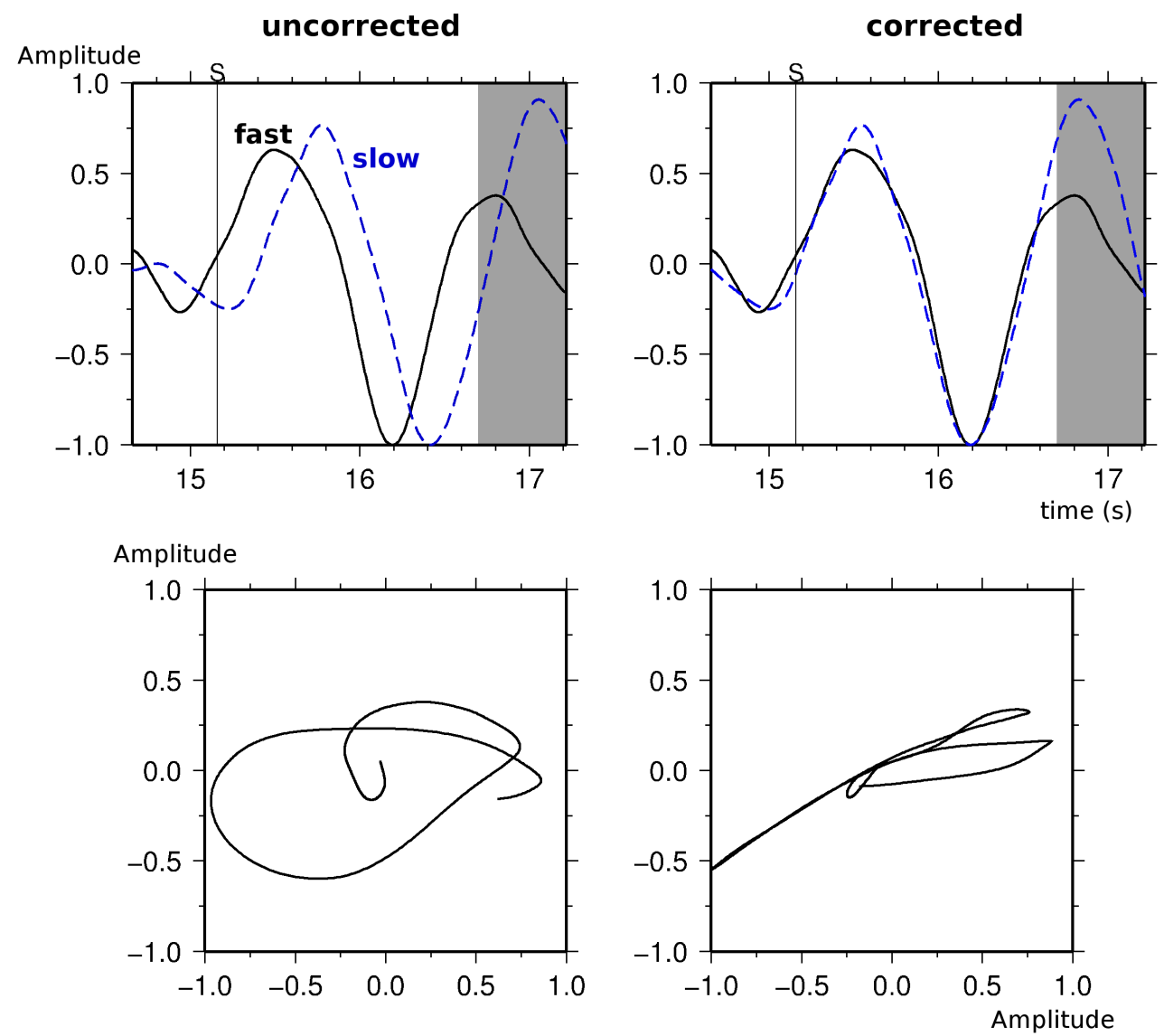

Figure 4.6.: Comparison of seismograms of a split wave before and after the correction. Anisotropy is corrected (reversed) by applying an inverse splitting operator. This figure shows an example for a good measurement: The waveforms match well after correction and the particle motion is almost linear. The event was recorded at the LHUT2 station of the CHARM deployment in January 2002 and was filtered with a $0.4-1 \mathrm{~Hz}$ bandpass filter (2002.016.12.02.lqua2.0.4-1). Amplitudes are of an arbitrary scale that has been normalised to \pm 1 .

Top: Fast and slow component in a composite plot. For the uncorrected seismograms a time lag between fast and slow component is clearly visible. The area with grey-shaded background is outside the chosen measurement window. The corrected wavelets show the similarity of the fast and slow component for this event.

Bottom: Particle motion of the fast and slow component. Correction results in an almost linear particle motion, because an un-split shear wave is polarised in only one orientation.

motion, while the corrected wave should show a particle motion that is more linear. The linearity of the particle motion, quantified by the value of $\lambda_{2}^{\min }$, is a criterion for the quality of a shear wave splitting measurement and the resulting splitting param- 


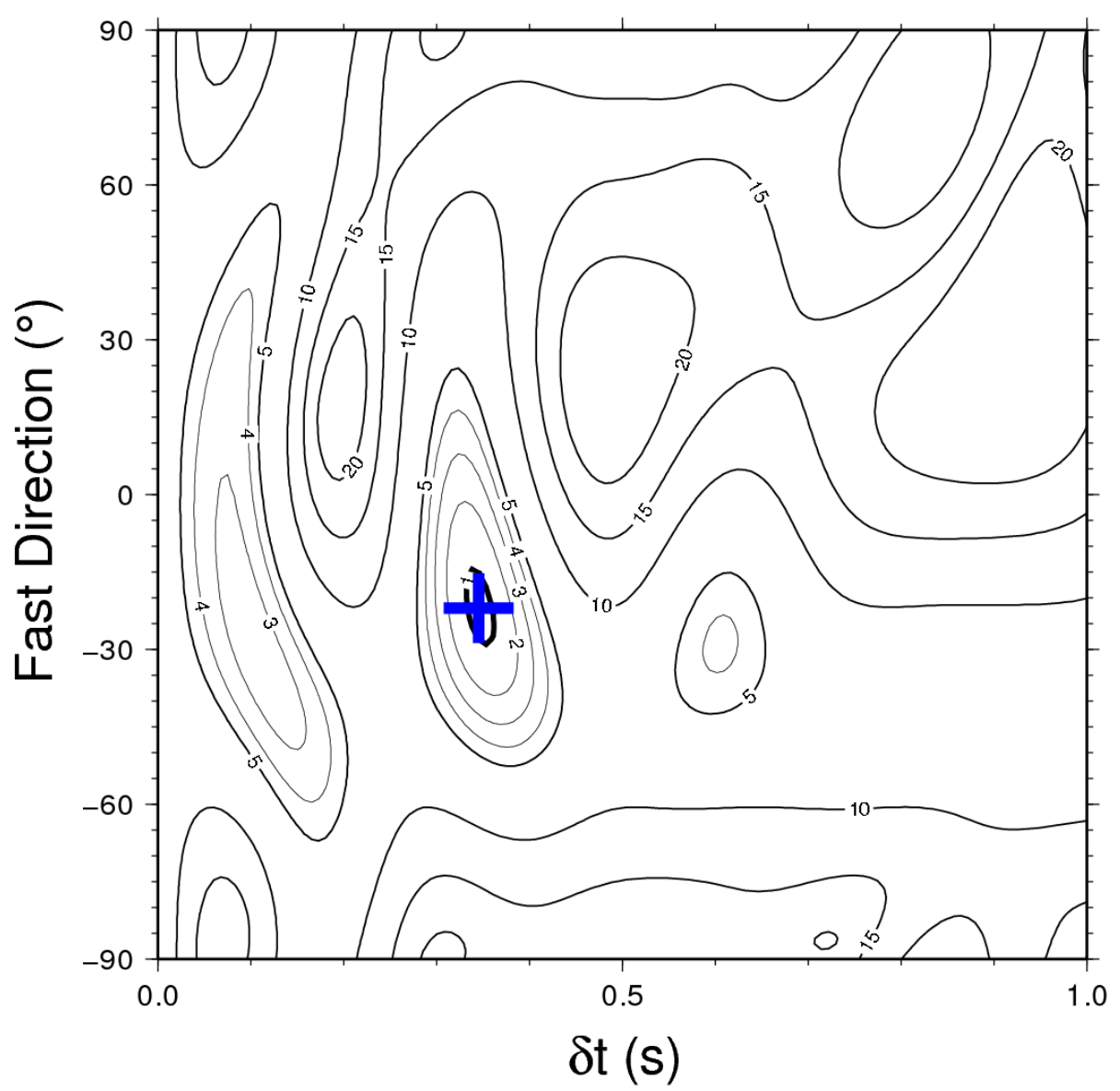

Figure 4.7.: Error surface of the grid search for the shear wave splitting parameters $\Phi$ and $\delta t$. The cross marks the solution of the grid search, the contour area of 1 (directly underneath the cross) marks the $95 \%$ confidence interval, other contour lines indicate multiples of this (Silver and Chan, 1991; Teanby et al., 2004b). The figure shows event 2005.002.TUVZ.2346346 filtered with a $0.2-2 \mathrm{~Hz}$ bandpass filter.

eters. A lower $\lambda_{2}^{\min }$ leads to a smaller confidence region, so that the uncertainties of the measurements are lower, too.

\subsubsection{Measurement window}

The part of the waveform that is used to perform the splitting measurements is hereafter referred to as the measurement window (Figure 4.8). This is not to be confused with the shear wave window, which describes a range of incident angles that are less than a critical incidence angle $i_{c}$ (see Section 3.2.1). 


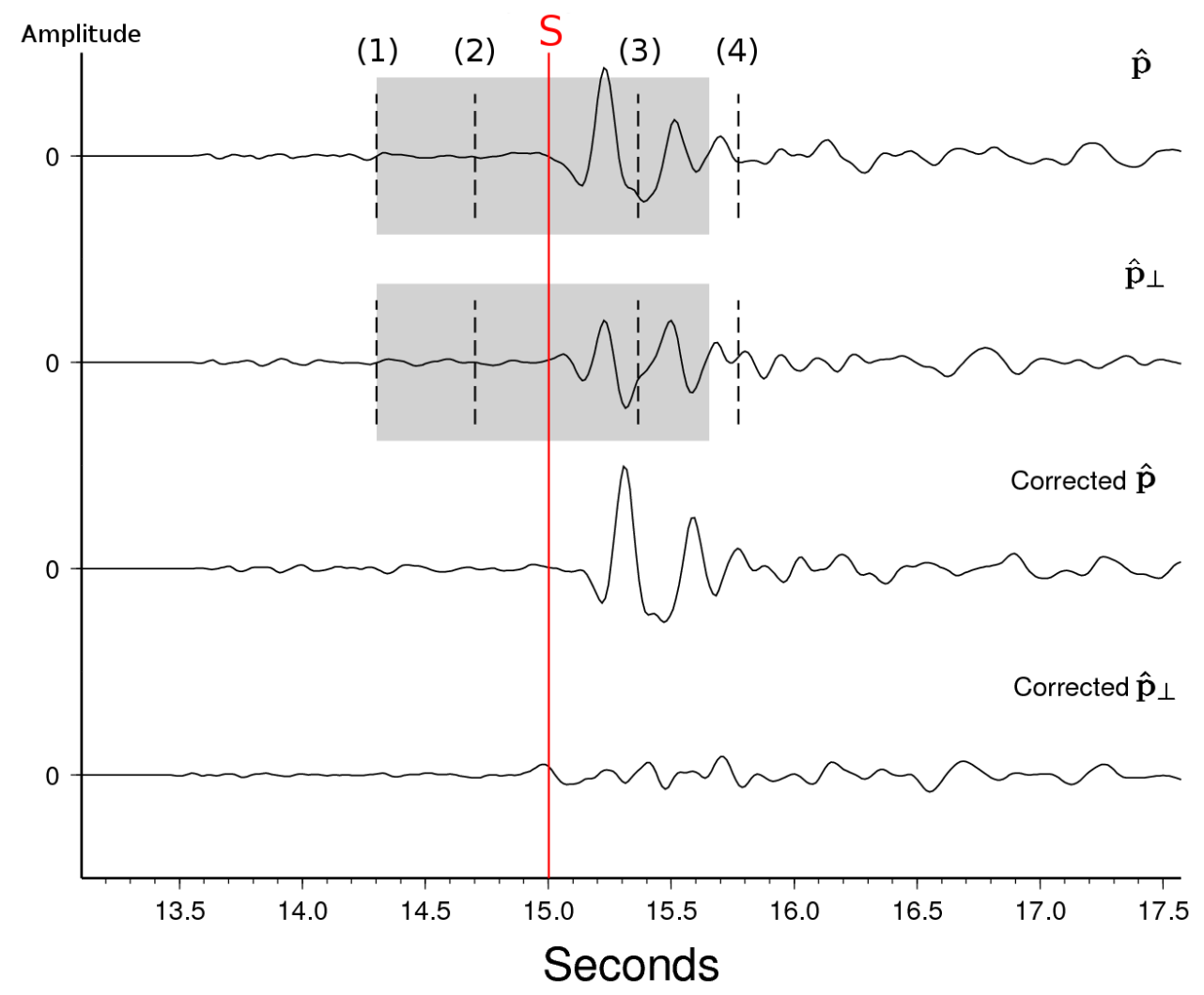

Figure 4.8.: Rotated seismograms before correction (top two traces). $\hat{\mathbf{p}}$ is the component orientated parallel to the initial polarisation, while $\hat{\mathbf{p}}_{\perp}$ is the component perpendicular to the initial polarisation. These seismograms are derived from the original east and north components by rotation. The vertical component is not shown. If a shear wave is not split, all its energy should remain on the $\hat{\mathbf{p}}$ component, while with the presence of shear wave splitting, an S-phase signal also appears on the perpendicular component $\hat{\mathbf{p}}_{\perp}$. A measurement window is defined by two time values, $t_{\text {beg }}$ and $t_{\text {end }}$. The cluster analysis code (see Section 4.3.3) cycles through several different measurement windows that begin between (1) and (2) and end between (3) and (4). The step size between these borders and the time values for (1)-(4) are read from a configuration file. For $n_{\text {beg }}$ window start times and $n_{\text {end }}$ window end times, a total number of $n=n_{\text {beg }} \cdot n_{\text {end }}$ windows will be considered. In this example 75 windows have been tested $\left(n_{\text {beg }}=3\right.$ and $\left.n_{\text {end }}=25\right)$. The measurement window that was finally selected after the cluster analysis is indicated by a grey shaded box.

The two bottom traces show the components parallel and perpendicular to the initial polarisation after the correction. The energy on the perpendicular component has been minimised by the algorithm. The figure shows event 2002.133.18.16.lhut2 filtered with a 1-3 Hz bandpass filter.

The measurement window is an important parameter and can have strong influence on the results of the measurement. With manual measurements it is often necessary 
to test if a slightly different window leads to different results. This is addressed by the cluster analysis method (see next section and Teanby et al., 2004b), which is used to perform measurements for a given number of different measurement windows and then tries to find the most stable solution.

\subsubsection{Cluster analysis}
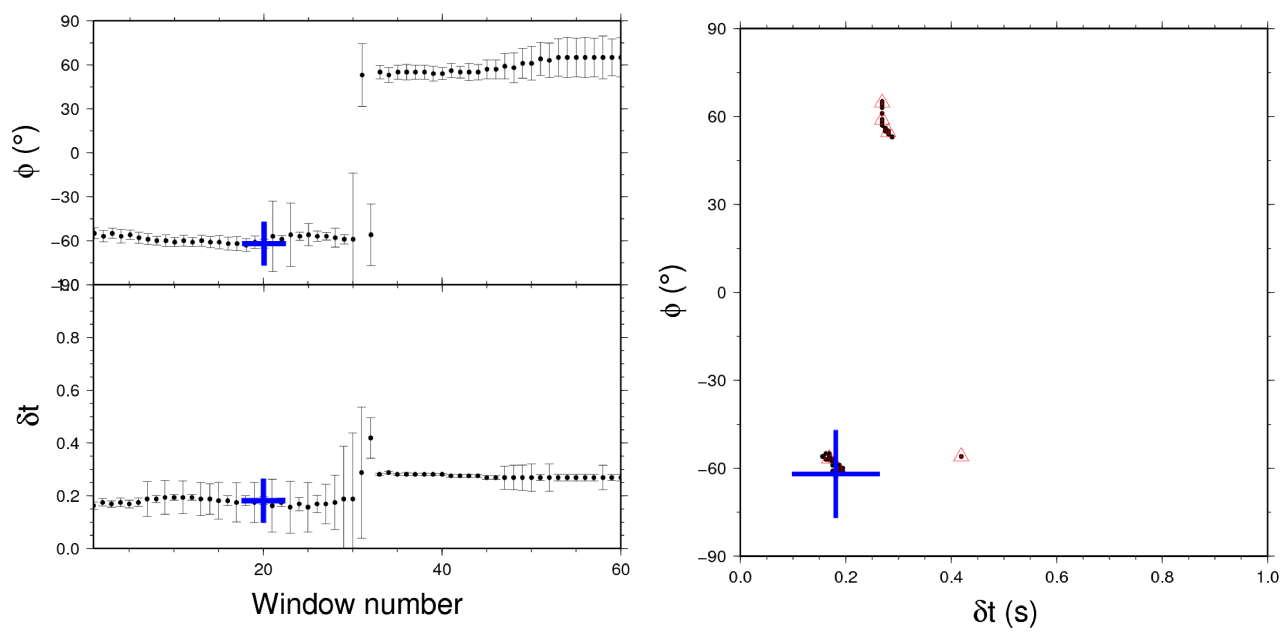

Figure 4.9.: Cluster analysis plots for a measurement of 2002 data from CHARM station LHUT2 (event 2002.015.09.21.lhut2 filtered with a 0.3-3 Hz bandpass filter). These plots illustrate the dependency of some splitting results on the measurement window.

Left: Overview of splitting measurement results for 60 different windows. This example demonstrates that the results can be sensitive to the choice of the measurement window and therefore illustrates the advantage of the cluster analysis method. Cycle skipping effects (see Section 3.2.2) are expected to lead to different results for different measurement window configurations and can be indicated by different groups of splitting parameters in the cluster analysis plots. In this case the results for windows 1-30 are significantly different from results for windows $31-60$. Note that higher window numbers represent larger measurement windows. The cluster analysis method addresses the problem of window-dependent results by performing multiple measurements for a range of windows and then choosing the most stable result. The chosen result is indicated by a cross in this figure.

Right: Cluster Analysis plot. Results for several measurement windows (here: 60) are grouped into clusters and the most stable result is chosen. The selected cluster is indicated by a cross. See text and Teanby et al. (2004b) for details. 
The cluster analysis method (Teanby et al., 2004b) is an improved shear wave splitting measurement method which is based on the algorithm of Silver \& Chan (see above). Instead of testing different measurement windows and their effect on the results manually, it cycles through a given set of windows, performs the splitting measurement for each window and searches for a stable solution by doing a cluster analysis. The cluster analysis method gave reliable results when applied to microseismic data from borehole seismometers (Teanby et al., 2004a,b), but it has to be noted that only a subset of data were processed in the study (namely those events that gave usable results in an earlier manual study). Sub-surface borehole data are also less affected by surface effects* as crustal events recorded by regular seismometers on the surface (Crampin and Gao, 2006). These problems should be considered when using the cluster analysis method with other data sets.

However, results obtained from manual measurements can suffer from instability and can indeed be dependent on the measurement window. A slightly different measurement window can yield significantly different results (see Figure 4.9). This problem is addressed by the cluster analysis method, where only measurements that give very similar results for a certain number of windows are considered as stable and therefore reliable.

The total number of measurement windows that are processed by the cluster analysis depends on the selected number of different window start and window end times: $n=n_{\text {beg }} \cdot n_{\text {end }}$

The process of measurement window configuration is illustrated in Figure 4.8 (see also Figure 4.9). The four time boundaries $t_{1}-t_{4}$ (earliest window start, latest window start, earliest window end, latest window end) are picked interactively on the screen or calculated from parameters in a configuration file. For the automatic measurement

\footnotetext{
* Surface effects can be caused by interfering surface wave arrivals or can cause splitting into non-orthogonal components. The latter is especially critical for non-normal incidence (Crampin and Gao, 2006).
} 
tool this is automatically done based on a calculation of the dominant period length of the data. An optimal measurement window end range should be from at least half a cycle after the S-pick to a maximum length that does not interfere with neighbouring phases (Teanby et al., 2004b). To account for events with different frequency content, the dominant period length $\bar{T}$ is calculated for a three second window directly after the S-pick for each event. After calculating the main frequency of the shear wave, the minimum and maximum window end times are derived, where the minimum window end time is by default set to $5 / 6 \cdot \bar{T}$ and maximum end time to $2.5 \cdot \bar{T}$. $^{*}$ These values were chosen from experience and prove to be a good compromise between allowing longer measurement windows that possibly suffer from interfering secondary phases and shorter windows that result in less stable measurements. The number of different window end times $n_{\text {end }}$ depends on the difference between $t_{3}$ and $t_{4}$ and is allowed to vary between 25 and 50 . Window start time limits are set to $t_{2}=t_{S}-0.3$ and $t_{1}=t_{S}-0.7 \mathrm{~s}$ and the number of different window start times is set to $n_{\text {beg }}=3$, because results tend to be less sensitive to the start time choice (Teanby et al., 2004b).

\subsubsection{Calculating the mean fast orientation}

The measured fast orientation $\Phi$ is a directional datum and has a bimodal distribution. The ambiguity is $180^{\circ}$, which means that $-90^{\circ}$ and $+90^{\circ}$ are equivalent. A single datum can be represented by a unity-length measurement vector $\Phi_{i}$ pointing into the measured direction. To obtain the mean orientation $\bar{\Phi}$ I calculate a vector $\vec{R}$ which is the normalised sum of the measurement vectors:

$$
\vec{R}=\left(\begin{array}{c}
\frac{1}{n} \sum_{i=1}^{n} \cos \left(l \Phi_{i}\right) \\
\frac{1}{n} \sum_{i=1}^{n} \sin \left(l \Phi_{i}\right)
\end{array}\right)
$$

\footnotetext{
*A small offset of $0.15 \mathrm{~s}$ is added to account for some slightly early S-picks.
} 
where $n$ is the number of measurements and $l=2$ because of the bimodal distribution of the data (Davis, 1986; Gerst, 2003; Gumiaux et al., 2003). The mean direction $\bar{\Phi}$ is then calculated from the vector $\vec{R}$ :

$$
\bar{\Phi}=\frac{\arctan \left(\frac{1}{n} \sum_{i=1}^{n} \sin \left(l \Phi_{i}\right) / \frac{1}{n} \sum_{i=1}^{n} \cos \left(l \Phi_{i}\right)\right)}{l} .
$$

The length $|\vec{R}|$ of the normalised vector $\vec{R}$ indicates the variance of the data set, where the minimum length 0 means high scattering of values and the maximum length 1 means high clustering (Peng and Ben-Zion, 2004). The vector length $|\vec{R}|$ and the number of measurements $n$ can be used to test the data for non-randomness (Davis, 1986). A vector length of $|\vec{R}|=0.3$ corresponds to a 90\%-confidence in non-randomness for 25 measurements and to a 99\%-confidence for 50 measurements. For a given number of measurements, the higher the vector length $|\vec{R}|$ the higher the confidence that the distribution of data is not random. And for a given vector length, the higher the number of measurements $n$ the higher the confidence in nonrandomness (Davis, 1986).

\subsection{Quality control}

To reject erroneous measurements automatically, quality criteria have been applied to reject measurements that do not meet them:

- signal to noise ratio above threshold $(\geq 3)$ to ensure good signal

- maximum error of $20^{\circ}$ for the fast orientation $\Phi$ in the chosen measurement window

- delay time not too close to the maximum allowed delay time (the maximum allowed delay time for the grid search was $1.0 \mathrm{~s}$, so that all measurements give a delay time of $0-1 s$. Cut-off criterion for delay times was $t_{\max }=0.8 \mathrm{~s}$ ) 
- initial polarisation not too close to the fast (or slow) orientation

By default, only events with a signal to noise ratio of three or greater are included in any further results, although events with $2 \leq S N R<3$ have been included in previous steps and can be considered for sparse data sets. Hereafter I use the terms "good" and "bad" measurements to distinguish between measurements that were accepted or rejected because of different qualities based on the mentioned criteria. The last two bullet points address the possible occurrence of null measurements (see Section 4.4.2).

\subsubsection{Cycle skipping}

Cycle skipping occurs when a split shear wave is erroneously fit:

- by shifting the slow component not only for the correct amount of $\delta t$, but also for another cycle of the waveform.

- by mistakenly switching the fast and slow shear wave and therefore shifting by the correct delay time plus an additional half cycle

For some data the fast and slow waveform can be in good agreement even with a skipped cycle. This can happen especially for sinusoidal events, which might occur when filtering with a bandpass of narrow frequency range. Cycle skipping introduces an error of $90^{\circ}$ (an "error" of $180^{\circ}$ and a high delay time are also possible) to the measured orientation of the fast shear wave. This error is of the same value that is expected for some crack-induced temporal changes in anisotropy (see Section 2.3) and therefore must be avoided.

Measurements with cycle skipping are expected to have a delay time that is larger than for measurements without this problem. Typically the delay time would be enlarged by a half of the dominant period (Matcham et al., 2000). Including parts of the seismogram before the S-phase arrival in the measurement window can help 
to prevent cycle skipping, because skipping of a cycle would then result in a larger misfit (see Figure 3.3, p.50 for illustration). Another diagnostic of cycle-skipped events is the contour plot of the error of the splitting parameters (Figure 4.7, p. 79), where in case of cycle skipping several minima in the contour plot are elongated along the $\Phi$-axis with a spacing of half a period length between them. If the choice of the measurement window has a large influence on the fast direction, this can also indicate cycle skipping.*

\subsubsection{Null measurements}

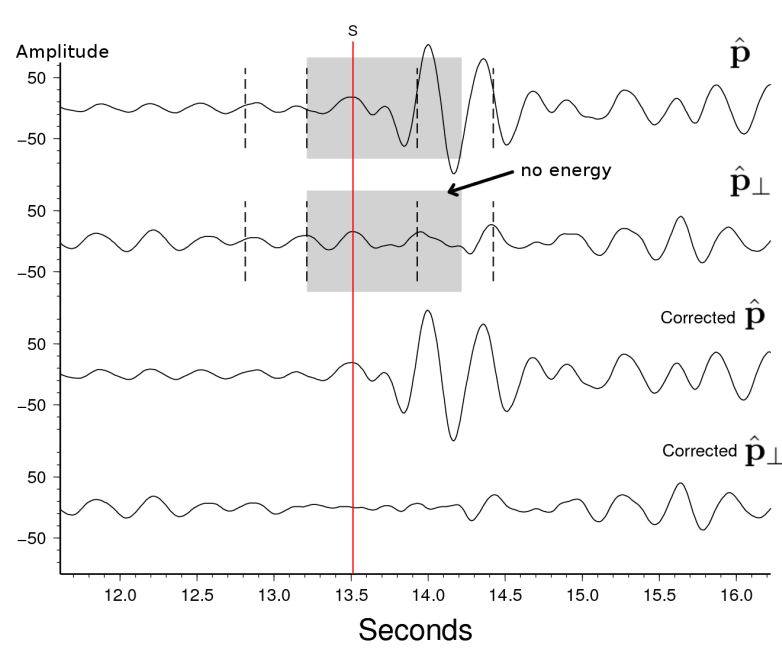

(a) Traces show the initial polarisationparallel and -perpendicular components before and after correction.

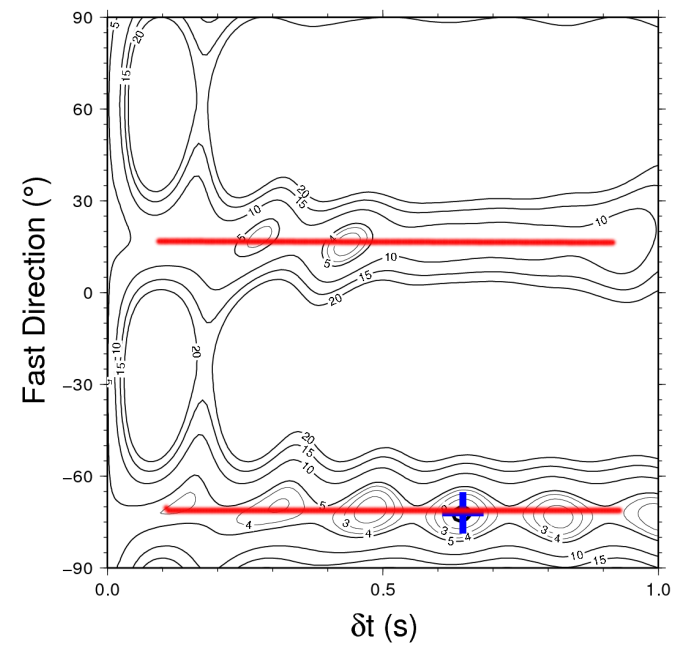

(b) Error surface.

Figure 4.10.: Null measurement (event 2005.002.TUVZ.2346225 filtered with a $2-3 \mathrm{~Hz}$ bandpass filter).

a) Components parallel $(\hat{p})$ and perpendicular $\left(\hat{p}_{\perp}\right)$ to the initial polarisation direction. Before (top) and after correction (bottom). The lack of energy on the perpendicular component indicates a null measurement.

b) Contour plot of the error surface of the same measurement. The red lines (indicating the most likely results) point out that for a null measurement the results show a $90^{\circ}$ ambiguity for the fast orientation and possibly lead to unusually large delay times. The $\subset$-shape of the contour lines is typical for null measurements.

\footnotetext{
*Figure 4.9 (p. 81) shows some results that possibly suffered from cycle skipping but were not selected by the cluster analysis.
} 
The definition and handling of null measurements (see Figure 4.10; also Section 3.2.2) is very important to get good results from shear wave splitting measurements. Following the suggestions of Levin et al. (2007), events that give a delay time close to the allowed maximum are possibly null measurements and are therefore rejected. An earlier study at Mt. Ruapehu reported mean delay times of $0.107 \mathrm{~s}$ for shallow and $0.272 \mathrm{~s}$ for deep events in data from 2002 and similar delay times in data from 1994 and 1998 (Gerst and Savage, 2004). Individual measurements often gave higher delay times, but from hundreds of measurements only a single one had a delay time of greater than $0.8 \mathrm{~s}$ and gave a good result (Gerst, 2003). Compared with this, numerous measurements that gave a delay time of greater than $0.8 \mathrm{~s}$ were null or low quality measurements. Therefore I reject all the high delay times by setting a reasonable maximum delay time $\delta t_{\max }=0.8 \mathrm{~s}$, chosen from experience. ${ }^{*}$ The risk of losing a few good events is compensated by the automated rejection of null measurements. However, if the data set is sparse, these rejected events can be investigated manually.

The use of a high maximum delay time has been suggested and was said to facilitate the detection of null measurements, cycle skipping and noisy events (Teanby et al., 2004b; Evans et al., 2006). I tested this hypothesis by using a maximum delay time of two seconds for some hundred measurements on several CHARM and GeoNet stations. To initially allow as much as two seconds delay time did not improve the results from data of this study. Instead of nulls and noisy events tending towards delay times close to the allowed maximum, many previously good measurements appear to be cycle skipped and give unreasonable high delay times, so that this approach has not been used.

Another way to find a possible null measurement is to compare the initial S-wave polarisation $\alpha$ with the measured fast orientation $\Phi$. This accounts for the possibility

\footnotetext{
*The maximum allowed delay time for the grid search is one second. The delay time for local events to investigate crustal anisotropy should not exceed 1 second, in fact it should usually be about $0.1-0.6$ seconds (see Table 1 of Savage, 1999, for a summary of many studies).
} 
that the S-wave is already polarised in the fast (or slow) orientation (see Figure 3.4). It is probably better to handle each measurement's $\Phi-\alpha$ pair separately instead of comparing the initial polarisations with an average fast orientation. This is especially true when dealing with scattered values of $\Phi$ (Peng and Ben-Zion, 2004), and this approach is followed in this study. Measurements that do not meet the criterion

$$
\tau \leq|\Phi-\alpha| \leq 90-\tau
$$

where $\tau$ is a threshold (default is $20^{\circ}$, following Peng and Ben-Zion), are considered as null measurements and treated separately for further interpretation.

\subsection{Illustrating the results}

Trends for many measurements can be shown by plotting the results in a rose diagram (distribution of the fast direction $\Phi$; see Figure 4.11), a density plot ( $\Phi$ or $\delta t$ vs depth) or a splitting map (single measurements as bars). Moving-window averaged results can be plotted to search for temporal changes of the parameters. The results of a single measurement can be illustrated in an analysis plot (Figure 4.12).

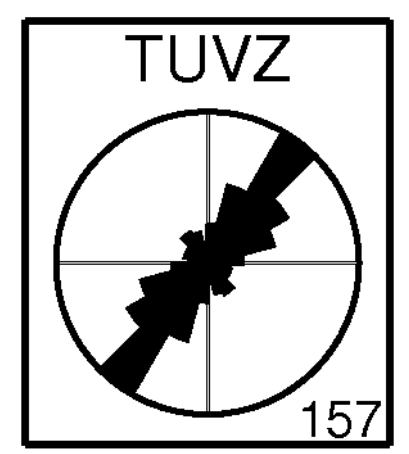

Figure 4.11.: Rose diagram for data from GeoNet station TUVZ for 2005 (deep events). The rose diagram shows the distribution of the fast orientations for deep events $(\geq 55 \mathrm{~km})$. The number to the lower right denotes the number of measurements that are shown in the rose diagram. The sector width of this and all following rose diagrams is $15^{\circ}$ unless otherwise noted. 

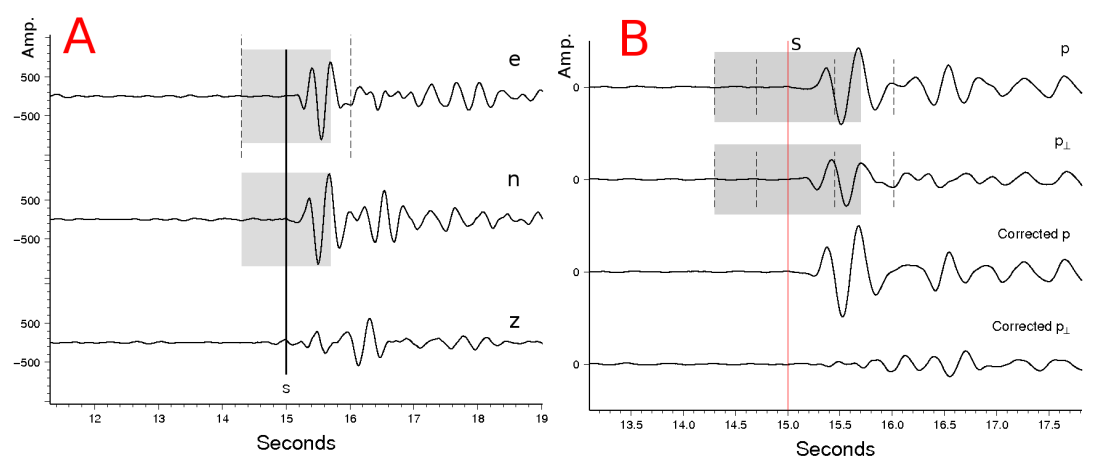

C
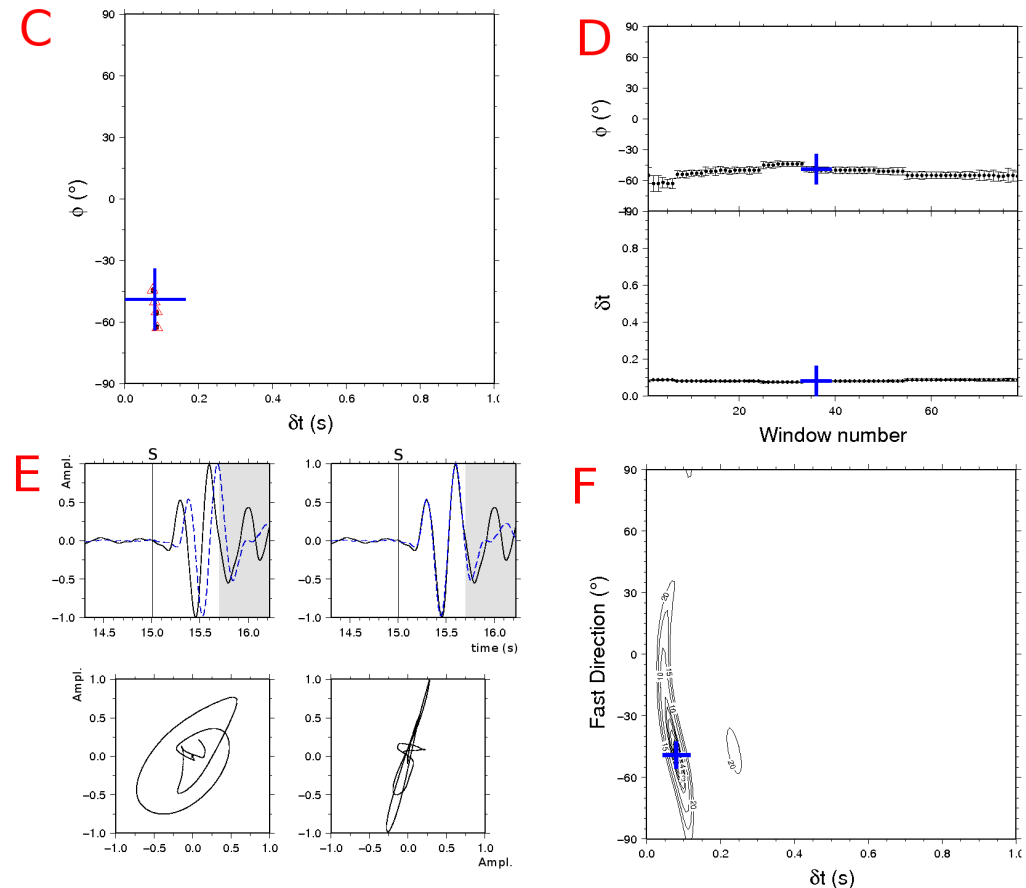

G

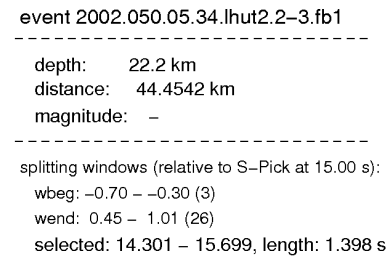

results:

$$
\begin{aligned}
& \text { fast: } 131.0+/-2.0\left(^{\circ}\right) \\
& \delta t=0.081+/-0.002(\mathrm{~s}) \\
& \text { spol: } 15.7+/-0.3\left(^{\circ}\right) \\
& \text { fast (abc): }-49.0+/-2.0\left(^{\circ}\right)
\end{aligned}
$$

Figure 4.12.: Analysis plot for a single shear wave splitting measurement (event 2002.050.05.34.1hut2.2-3). The figure is composed of six subfigures and a textbox, showing event details and measurement results.

A) Three components of the seismogram and S-pick. The measurement window that was selected by the cluster analysis is indicated by a grey shaded box.

B) Rotated components (parallel and perpendicular to the initial polarisation) before and after correction. See Figure 4.8.

C,D) Cluster analysis plots. See Figure 4.9.

E) Waveform and particle motion uncorrected (left) and corrected for splitting. See Figure 4.6 (p. 78).

F) Contour plot of the error of the splitting parameters. See Figure 4.7.

G) Event details and measurement results. 


\subsection{Routine automatic measurements and monitoring}

A main goal of this study is the development of an automatic measurement tool to routinely monitor changes in anisotropy at Mt. Ruapehu volcano. Full automatic measurements of shear wave splitting are at least difficult to carry out, because particle motion of the split S-waves is complex and results can have large scatter (Section 3.3; Elkibbi and Rial, 2005; Gao et al., 2006). There are four distinct groups of tasks that are laborious and time-consuming, if done manually:

1. data preparation

2. shear wave splitting measurement (incl. selection of measurement windows)

3. quality control

4. publication

While some recurring tasks can easily be automated, for some other steps the automation is not trivial. The new automatic measurement tool works without any manual input from a user and all results are reproducible. It has been optimised for the use with Geonet data to monitor shear wave splitting at Mt. Ruapehu (Appendix A.3). Given a list of earthquakes, data are retrieved from the Geonet FTP server and converted into a convenient format, a splitting measurement is performed, and the results are evaluated and displayed. These steps were briefly described in the previous sections. For a detailed technical description of the software see Appendix A, p. 143. 


\section{Data used for shear wave splitting measurements}

In this section I describe the data that were used during this study. This study of changes in seismic anisotropy is not dependent on local seismicity at Mt. Ruapehu, because regional tectonic earthquakes are used to measure the shear wave splitting. Recent data are collected by permanent three-component GeoNet stations* and the stations adjacent to Mt. Ruapehu, listed in this chapter.

\subsection{Data from previous studies}

The available parts of the data sets from 1994 (deep events), 1998 (shallow events) and 2002 (all events) have been reprocessed for comparison of the previously used manual method with the newly developed automatic technique. Given the sparse data sets of the 1994 and 1998 events, these data sets have not been processed for single stations but as a whole.

\subsubsection{The 2002 CHARM deployment}

Because of the different station locations in 1994 compared to 1998 and a four year data gap, the CHARM deployment was initiated in 2002 to investigate the possibility

*Website: http://www.geonet.org.nz, visited August 10, 2007. 
of temporal changes in anisotropy at Mt. Ruapehu in greater detail. The station locations were chosen to reoccupy locations from the previous deployments (Figure 3.7, p. 67) to better distinguish temporal changes in anisotropy from heterogeneities in the investigated area (Gerst, 2003; Gerst and Savage, 2004). One permanent GeoNet station (FWVZ) installed at the Whakapapa ski field, about $3 \mathrm{~km} \mathrm{~N}-\mathrm{NW}$ of Crater Lake, also contributed data to the 2002 data set.

\subsection{GeoNet data}

All new data analysed in this study were recorded with GeoNet network stations (see Chapter 1.3) and were supplied by and retrieved from GeoNet servers. Single events are identified by an event-specific CUSP ID (Caltech-USGS Seismic Processing), a 7-digit number that is assigned by GeoNet and is unique to every event. The data were made available as "Standard for the Exchange of Earthquake Data" (SEED) files, where one SEED file contains data of one event from all stations where it was recorded. The seismograms are typically 40-60 seconds long.

For this study only the stations in the vicinity of Mt. Ruapehu were of interest (Figure 5.1). Six three-component stations are positioned within $10 \mathrm{~km}$ of Crater Lake and several more stations are located within $20 \mathrm{~km}$. The stations are permanently deployed and are operated by the GeoNet project. See Table 5.1 for details and Figure 5.2 for an overview of station run times.

\subsubsection{GeoNet data event details}

The magnitudes in the GeoNet data set are local magnitudes $M_{L}$, derived from averaging of estimates from several stations (Haines, 1981; Robinson, 1987).* Only

*This is described in greater detail at http://www.geonet.org.nz/resources/ earthquake/hypocentre-derivation.html, visited July 10, 2007. 


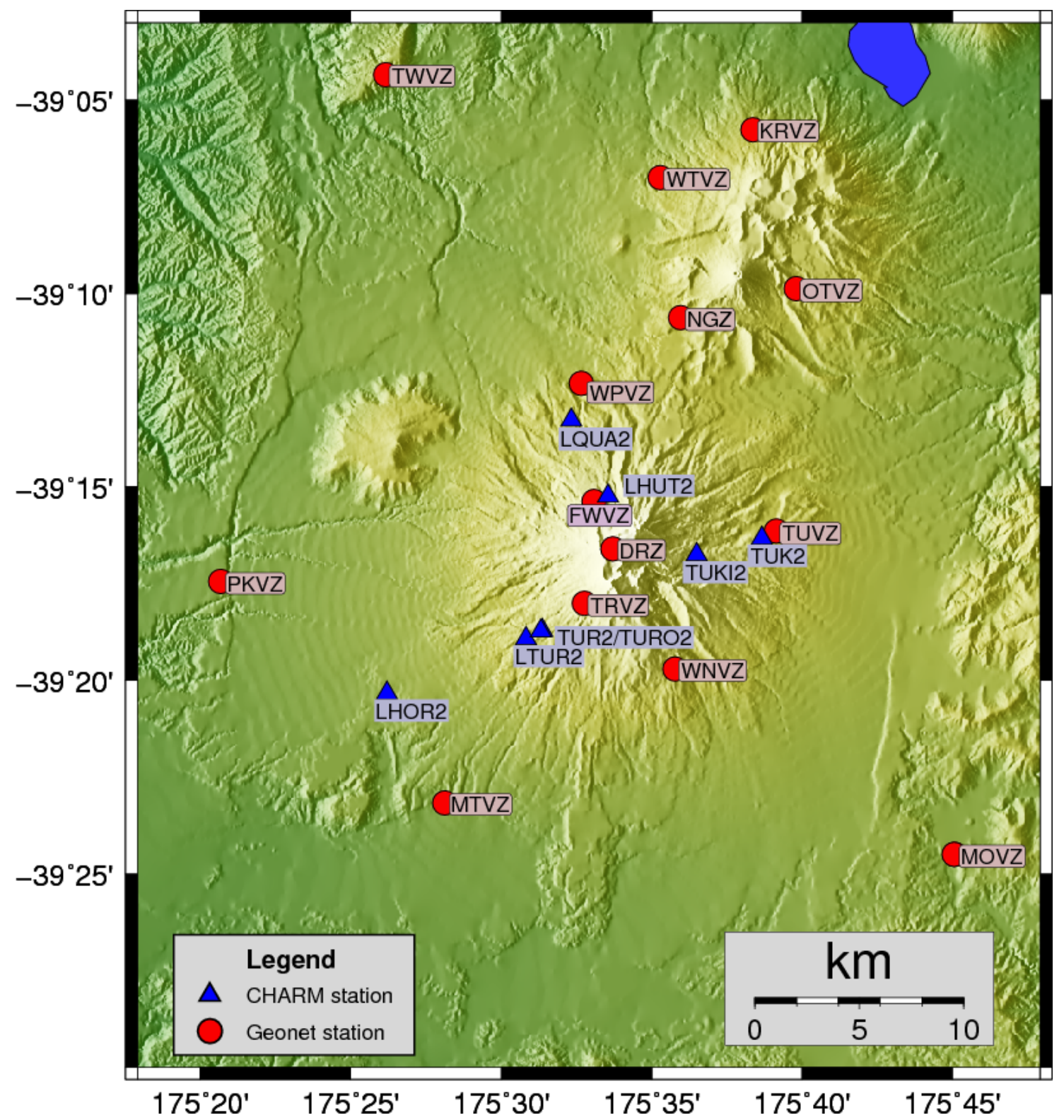

Figure 5.1.: Three-component stations of the GeoNet network at Mt. Ruapehu as of December 2007. Stations of the temporary CHARM deployment (2002) are shown for comparison. 


\begin{tabular}{|l||c|r|r|l|c|}
\hline Name & Latitude & Longitude & $\begin{array}{r}\text { Alt. } \\
(\mathrm{m})\end{array}$ & $\begin{array}{l}\text { opened } \\
\text { (3-comp. })\end{array}$ & $\begin{array}{l}\text { dist. } \\
(\mathrm{km})\end{array}$ \\
\hline \hline DRZ & -39.27703 & 175.56335 & 2692 & $2002 / 02 / 18$ & $\leq 1$ \\
TRVZ & -39.30057 & 175.54764 & 2062 & $2005 / 03 / 16$ & 2.6 \\
FWVZ & -39.25670 & 175.55276 & 2043 & $1999 / 07 / 07$ & 2.9 \\
\hline WNVZ & -39.32873 & 175.59769 & 1566 & $2004 / 05 / 04$ & 6.0 \\
TUVZ & -39.26943 & 175.65398 & 1446 & $2004 / 06 / 01$ & 7.9 \\
WPVZ & -39.20576 & 175.54575 & 1244 & $2003 / 12 / 10$ & 8.5 \\
\hline NGZ & -39.17734 & 175.60098 & 1452 & $2004 / 03 / 04$ & 12.0 \\
MTVZ & -39.38636 & 175.47023 & 840 & $2005 / 03 / 15$ & 14.2 \\
OTVZ & -39.16487 & 175.66489 & 1506 & $2004 / 11 / 18$ & 15.6 \\
WTVZ & -39.11701 & 175.58951 & 1186 & $2004 / 03 / 04$ & 18.4 \\
PKVZ & -39.29110 & 175.34610 & 770 & $2005 / 03 / 15$ & 18.8 \\
MOVZ & -39.40850 & 175.75266 & 874 & $2004 / 06 / 01$ & 21.5 \\
TWVZ & -39.07250 & 175.43750 & 1084 & $2004 / 05 / 18$ & 25.6 \\
\hline
\end{tabular}

Table 5.1.: Stations of the GeoNet network that provided data for this study. All stations are running to date (December 2007). The stations are sorted by distance from Crater Lake. Stations MOVZ and TWVZ are more than $20 \mathrm{~km}$ away but have been included. The lines indicate three groups of stations, according to their distance from Crater Lake (within $5 \mathrm{~km}$, within $10 \mathrm{~km}$ and within $20 \mathrm{~km}$ plus stations MOVZ and TWVZ). 


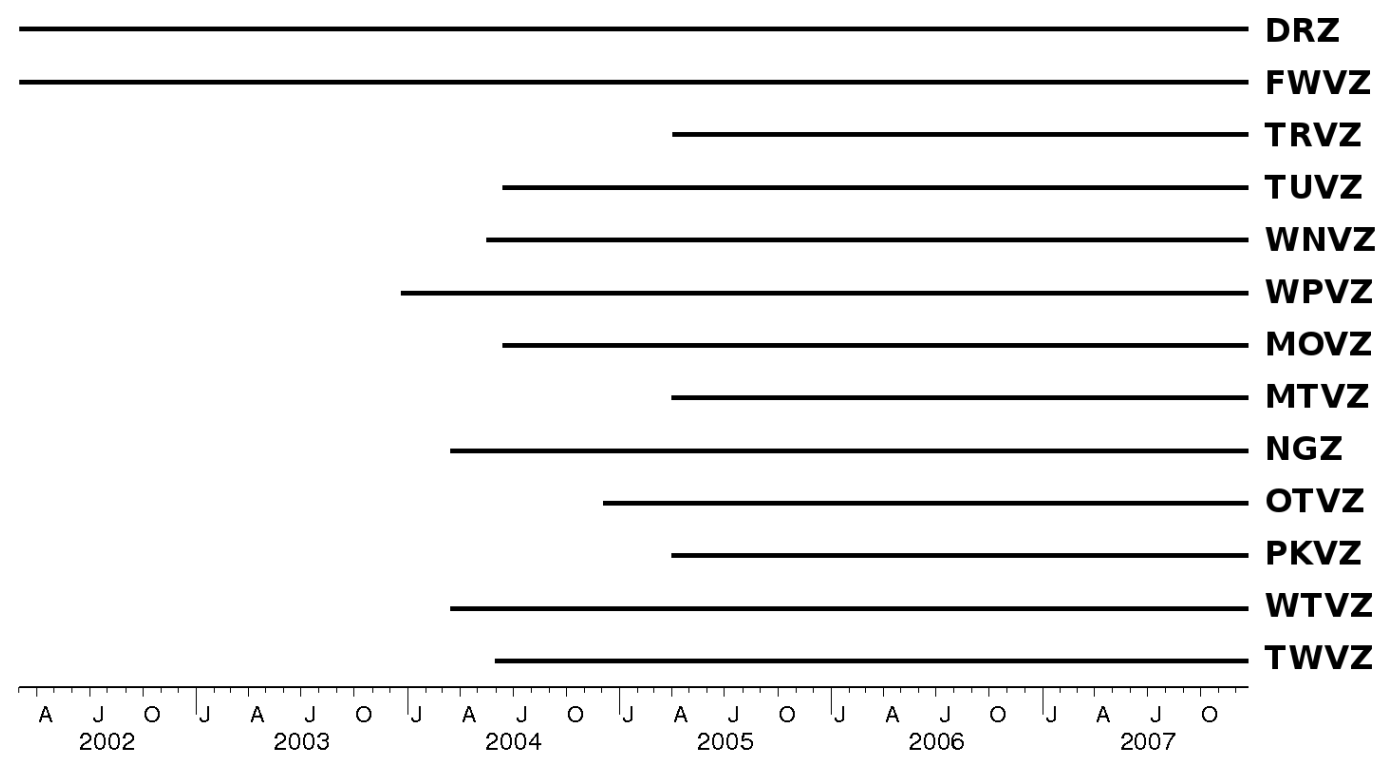

Figure 5.2.: Run times of GeoNet stations (with a three-component seismometer) in the vicinity of Mt. Ruapehu. The additional three-component stations that were installed in 2004 and 2005 make shear wave splitting measurements for this study possible without another temporary station deployment.

events with a magnitude $M_{L} \geq 2$ and a maximum distance of $150 \mathrm{~km}$ from the summit of Mt. Ruapehu have been considered for analysis (Figure 5.3, p. 96). 

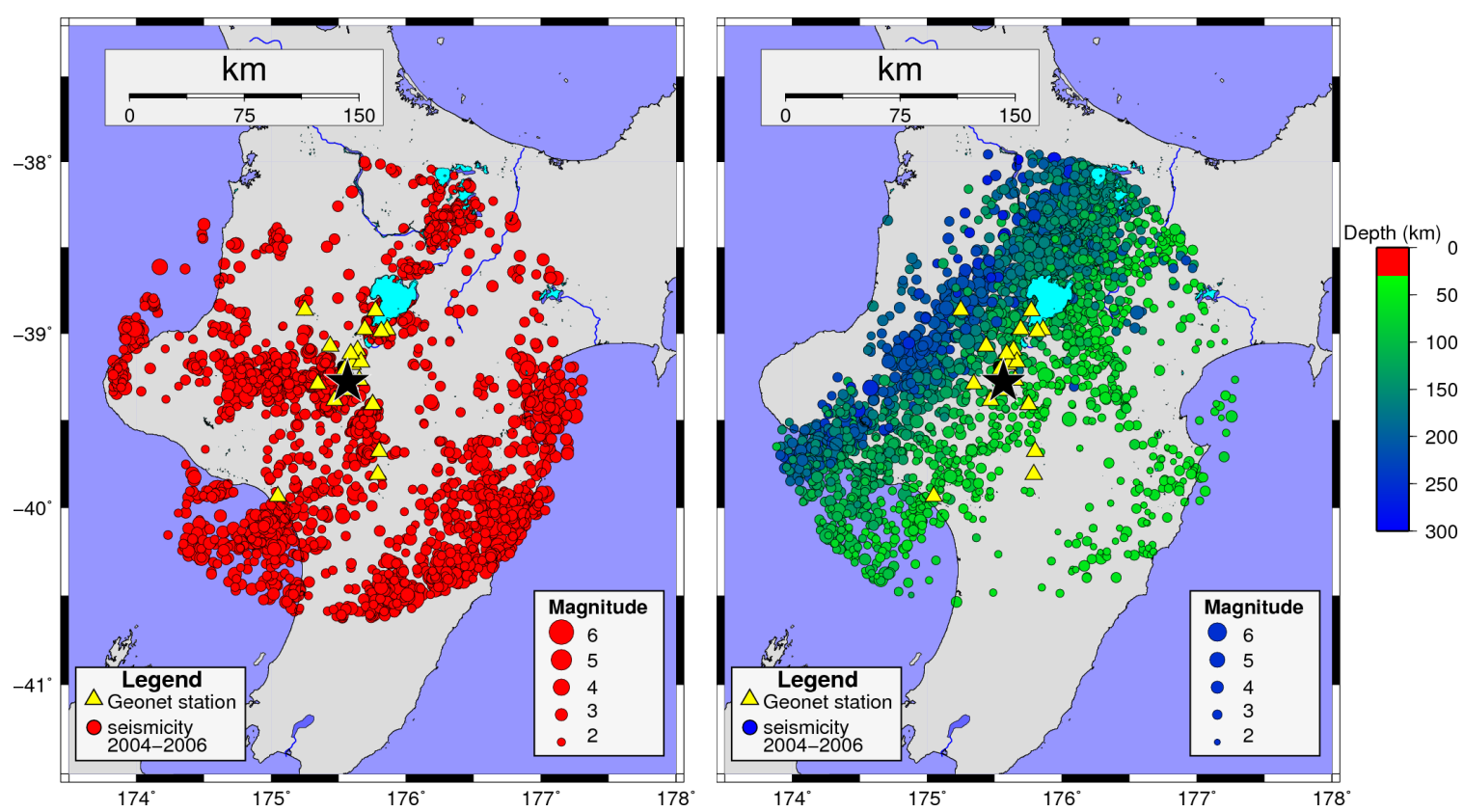

Figure 5.3.: Selected event data in 2004-2006 with a hypocentral distance of up to $150 \mathrm{~km}$ from Ruapehu and a magnitude of 2 or greater. The figure shows a total of 8502 events that passed the spatial selection criteria. Left: Shallow events $(0-30 \mathrm{~km})$. Right: Deep events $(55-300 \mathrm{~km})$. A larger map with event locations is shown in the Appendix (Figure B.1, p. 161). Only the data from stations in the vicinity of Mt. Ruapehu have been analysed. See Table 5.1 for station details. 


\section{Results}

\subsection{Development of an automatic measurement technique}

A main goal of this study was the development of an automatic measurement tool to facilitate near-real-time monitoring of seismic anisotropy and its temporal changes. The tool helps with and simplifies the following tasks:

- data processing preparation

- shear wave splitting measurement

- quality control steps

- results representation

The programs that have been developed for this study allow automatic shear wave splitting measurements and enable us to process hundreds and even thousands of events in reasonable time scales and with minimum manual intervention. Several parameters and a set of filters (from which the best filters are chosen for each event) have been chosen from experience. The results obtained from these automatic measurements are objective and exactly reproducible. The method is described in detail in Chapter 4 (general description of the method) and in Appendix A.4 (technical manual). The method is compared with other methods in Section 7.1.1, p. 129. 


\subsection{Shear wave splitting measurements}

The method for automatic shear wave splitting measurements has been applied to data from previous studies (Section 6.2.1) and to new data that has been collected since 2002 (Section 6.2.2). The method has furthermore been used to reprocess data from another deployment on the North Island of New Zealand (Savage et al., 2007).

\subsubsection{Reprocessing data from previous studies}

Data from deployments in 1994, 1998 and 2002 were analysed and temporal changes in anisotropy were found and published (Miller and Savage, 2001; Gerst and Savage, 2004). The data from these previous studies were reprocessed with the new automatic technique to evaluate its reliability. The original results from these previous studies are reviewed in Section 3.4.5.

To determine the best parameters and to test if the method is generally working, 20 high-quality events from the 2002 CHARM data set were selected and the splitting parameters were compared for manual and automatic technique (see Figure 6.1). Both fast orientation and delay times are reproduced for most events. Four events were not processed because of a too low signal to noise ratio. Of the 16 remaining events, three events are rejected by the following quality control criteria after the measurement. (In this case they are rejected because of too high delay times. See Section 4.4.) At least one of the three rejected events was most likely cycle-skipped, as the fast orientation shows a $\sim 90^{\circ}$-discrepancy between automatic and manual measurement. This leaves 13 measurements obtained with the automatic method, out of which 12 are in good agreement with results from the manual technique.

After parameters had been determined (see Section 4 for details) by the application of the automatic technique to a small set of selected high-quality events, the method was applied to complete data sets that include noisy data and null measurements. 


\section{Phi}

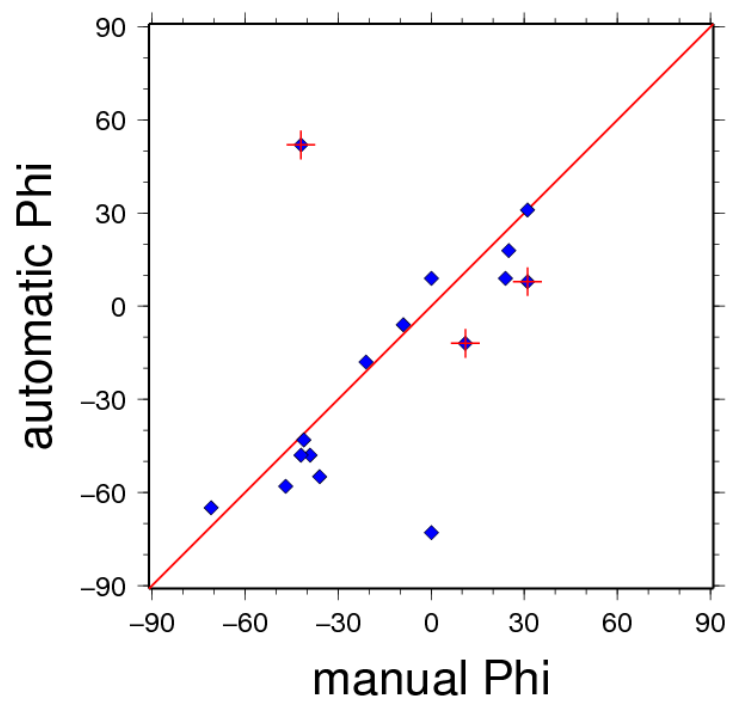

dt

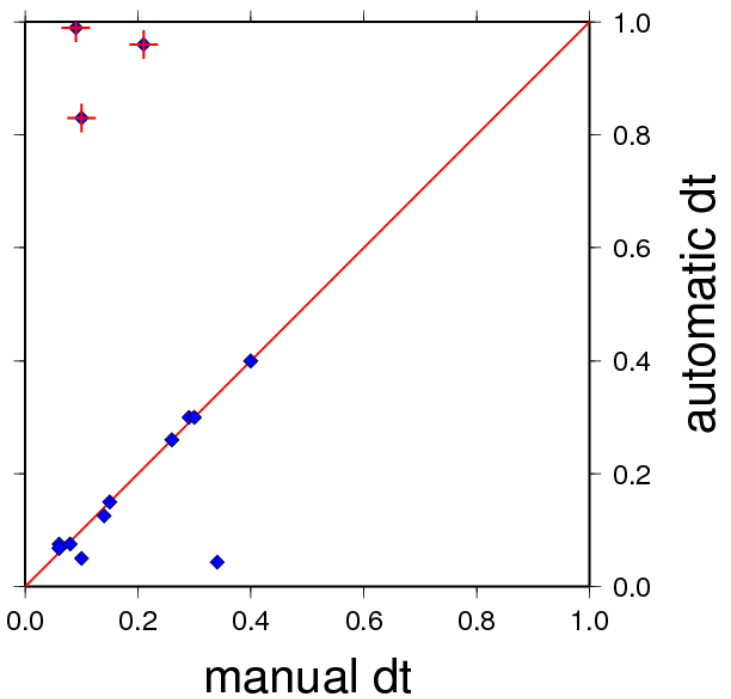

Figure 6.1.: Comparison of results obtained from manual and automatic (this study) shear wave splitting method in a scatter plot. Manual results are from Gerst (2003). 16 events that gave high-quality results for stations LHUT2, LQUA2, LHOR2 and TURO2 (2002 data) were selected. The 2002 data were used to find the best parameters for the automatic measurement technique. Fast orientations $\Phi$ are given in degree, delay times in seconds. Events marked by a cross were rejected by automatic quality control criteria (here: maximum delay time). Even though delay times are scattered at Ruapehu (Gerst and Savage, 2004) and in general for crustal events (Crampin and Gao, 2006), the right scatter plot suggests that they are still reproducible for single measurements.

Without the pre-selection step of only choosing high-quality events, results are naturally more scattered, as noisy events and disturbed waveforms are more likely to introduce irregularities and errors to the automatic processing (see following results).

\section{Results from the 1994, 1998 and 2002 deployments}

Only 26 distinct events from the 1994 deployment were available. By considering recordings at multiple stations and possibly more than one filter for each recording, Gerst (2003) obtained 60 measurements, while the automatic technique obtains 50. From 61 shallow events from the 1998 deployment, Gerst obtained 110 measurements, 
compared to 116 measurements with the automatic technique.* The results from events of the 1994 and 1998 deployment are shown in Table 6.1 and Figure 6.2. Results from the reprocessing of 2002 data are shown in Table 6.2 and Figures 6.36.5 .

\begin{tabular}{|l|l||r|r|r|r|r|r||}
\hline data set & method & \multicolumn{1}{|c|}{$\bar{\Phi}\left[^{\circ}\right]$} & $\pm \bar{\Phi}\left[^{\circ}\right]$ & $\overline{\delta t}[\mathrm{~s}]$ & $\pm \delta t[\mathrm{~s}]$ & $|\vec{R}|$ & $\#$ \\
\hline \hline \multirow{2}{*}{1994 deep } & automatic & $\mathbf{- 4 6 . 6}$ & $\mathbf{8 . 1}$ & $\mathbf{0 . 3 0}$ & $\mathbf{0 . 1 5}$ & $\mathbf{0 . 3 7}$ & $\mathbf{4 3}$ \\
& manual & $\mathbf{- 4 2 . 8}$ & 3.6 & 0.23 & 0.13 & - & 37 \\
\hline \multirow{2}{*}{1998 shallow } & automatic & -6.6 & 7.1 & 0.19 & 0.16 & 0.28 & 96 \\
& manual & 13.4 & 5.8 & 0.11 & 0.06 & - & 39 \\
\hline \hline
\end{tabular}

Table 6.1.: Results from the 1994 and 1998 deployments (all stations). Each entry consists of the mean fast orientation of all measurements $\bar{\Phi}$ and its standard error $\pm \bar{\Phi}$, the mean delay time $\overline{\delta t}$ and the standard deviation of the delay times $\pm \delta t$, the mean resultant length $|\vec{R}|$ and the number of measurements \#. Data sets with at least 20 measurements and a mean resultant length of $|\vec{R}| \geq 0.3$ are emphasised by bold font. Manual results of Gerst and Savage (2004) are shown for comparison for 1994 and 1998 data.

1994, deep

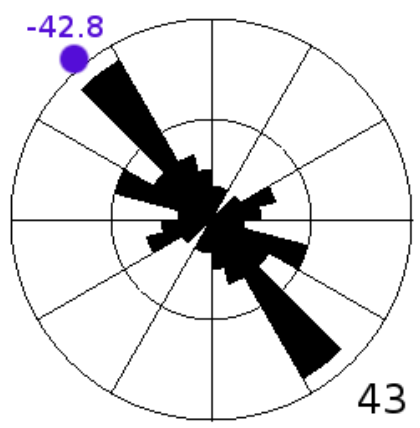

1998, shallow

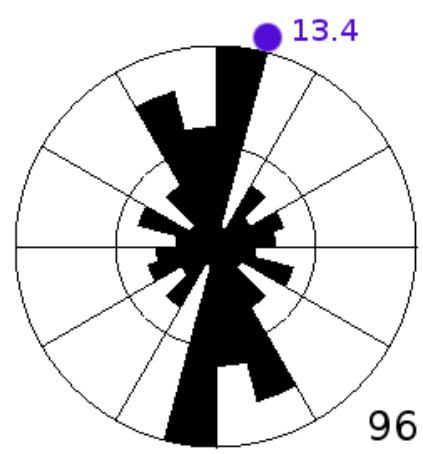

Figure 6.2.: Histograms of the fast orientation $\Phi$ for all stations from the 1994 (deep events) and 1998 deployment (shallow events). The number to the lower right of each rose diagram in this and all following figures denotes the number of measurements that are shown in the diagram. The results (mean fast orientation $\bar{\Phi}$ ) of Gerst and Savage (2004) are annotated on the rose diagram for comparison.

${ }^{*}$ These numbers include null measurements. See Tables 6.1 and 6.2 for detailed measurement numbers. 


\begin{tabular}{|c|c|c|c|c|c|c|c|}
\hline data set & method & $\bar{\Phi}\left[^{\circ}\right]$ & $\pm \bar{\Phi}\left[^{\circ}\right]$ & $\overline{\delta t}[\mathrm{~s}]$ & $\pm \delta t[\mathrm{~s}]$ & $|\vec{R}|$ & $\#$ \\
\hline shallow FWVZ & aut.fb1 & 2.1 & 8.6 & 0.09 & 0.07 & 0.70 & 8 \\
\hline shallow LHOR2 & aut.fb1 & -25.4 & 9.8 & 0.17 & 0.07 & 0.61 & 9 \\
\hline shallow LHUT2 & aut.fb1 & -46.4 & 8.2 & 0.14 & 0.12 & 0.65 & 11 \\
\hline shallow LQUA2 & aut.fb1 & -12.8 & 12.3 & 0.15 & 0.07 & 0.47 & 11 \\
\hline shallow LTUR2 & aut.fb1 & -2.5 & 4.7 & 0.12 & 0.08 & 0.85 & 12 \\
\hline shallow TUK2 & aut.fb1 & 41.0 & 9.3 & 0.24 & 0.06 & 0.90 & 2 \\
\hline shallow TUKI2 & aut.fb1 & -58.6 & 10.3 & 0.24 & 0.14 & 0.61 & 8 \\
\hline shallow TURO2 & aut.fb1 & -13.1 & 16.2 & 0.17 & 0.10 & 0.35 & 12 \\
\hline deep FWVZ & aut.fb1 & 61.1 & 33.0 & 0.33 & 0.11 & 0.19 & 10 \\
\hline deep LHOR2 & aut.fb1 & -10.5 & 16.1 & 0.18 & 0.12 & 0.33 & 14 \\
\hline deep LHUT2 & aut.fb1 & 38.9 & 15.2 & 0.30 & 0.16 & 0.37 & 12 \\
\hline deep LQUA2 & aut.fb1 & 37.5 & 14.8 & 0.40 & 0.17 & 0.43 & 9 \\
\hline deep LTUR2 & aut.fb1 & -5.9 & 26.8 & 0.28 & 0.13 & 0.22 & 12 \\
\hline deep TUK2 & aut.fb1 & -18.3 & 30.8 & 0.38 & 0.27 & 0.37 & 3 \\
\hline deep TUKI2 & aut.fb1 & 50.6 & 72.0 & 0.26 & 0.21 & 0.12 & 5 \\
\hline deep TURO2 & aut.fb1 & 34.2 & 19.2 & 0.29 & 0.13 & 0.31 & 11 \\
\hline shallow FWVZ & aut.fb3 & 1.5 & 7.2 & 0.15 & 0.14 & 0.52 & 25 \\
\hline shallow LHOR2 & aut.fb3 & -15.5 & 6.6 & 0.17 & 0.13 & 0.50 & 32 \\
\hline shallow LHUT2 & aut.fb3 & -49.6 & 5.0 & 0.11 & 0.09 & 0.66 & 27 \\
\hline shallow LQUA2 & aut.fb3 & -6.4 & 9.3 & 0.22 & 0.21 & 0.37 & 32 \\
\hline shallow LTUR2 & aut.fb3 & 1.7 & 4.8 & 0.15 & 0.15 & 0.59 & 40 \\
\hline shallow TUK2 & aut.fb3 & 34.9 & 10.7 & 0.24 & 0.08 & 0.70 & 5 \\
\hline shallow TUKI2 & aut.fb3 & -67.4 & 9.4 & 0.21 & 0.17 & 0.45 & 20 \\
\hline shallow TURO2 & aut.fb3 & -19.0 & 9.3 & 0.16 & 0.09 & 0.35 & 37 \\
\hline deep FWVZ & aut.fb3 & 45.8 & 9.1 & 0.35 & 0.14 & 0.41 & 26 \\
\hline deep LHOR2 & aut.fb3 & -13.8 & 8.1 & 0.18 & 0.11 & 0.39 & 38 \\
\hline deep LHUT2 & aut.fb3 & 40.5 & 7.2 & 0.28 & 0.18 & 0.49 & 29 \\
\hline deep LQUA2 & aut.fb3 & 23.5 & 8.6 & 0.43 & 0.18 & 0.45 & 25 \\
\hline deep LTUR2 & aut.fb3 & 4.4 & 17.0 & 0.25 & 0.13 & 0.21 & 33 \\
\hline deep TUK2 & aut.fb3 & 3.6 & 14.6 & 0.35 & 0.23 & 0.46 & 8 \\
\hline deep TUKI2 & aut.fb3 & 58.0 & 32.3 & 0.29 & 0.24 & 0.16 & 16 \\
\hline deep TURO2 & aut.fb3 & 10.2 & 13.0 & 0.26 & 0.13 & 0.27 & 31 \\
\hline shallow ALL & aut.fb1 & -18.1 & 5.3 & 0.16 & 0.11 & 0.43 & 73 \\
\hline shallow ALL & aut.fb3 & -15.2 & 3.9 & 0.17 & 0.15 & 0.34 & 218 \\
\hline deep ALL & aut.fb1 & 23.8 & 12.1 & 0.29 & 0.16 & 0.19 & 76 \\
\hline deep ALL & aut.fb3 & 19.7 & 5.5 & 0.28 & 0.18 & 0.25 & 206 \\
\hline
\end{tabular}

Table 6.2.: Results from the 2002 deployment (single stations). Columns are the same as in Table 6.1. See Table 3.2, p. 66, for previous manual results of 2002 data. "aut.fb1" denotes results from the automatic technique where only one filter is used for each event, "aut.fb3" denotes results where up to three filters are considered. Since Gerst (2003) also used more than one filter per event, the "aut.fb3" results are used to compare the results of both methods (Chapter 7). 


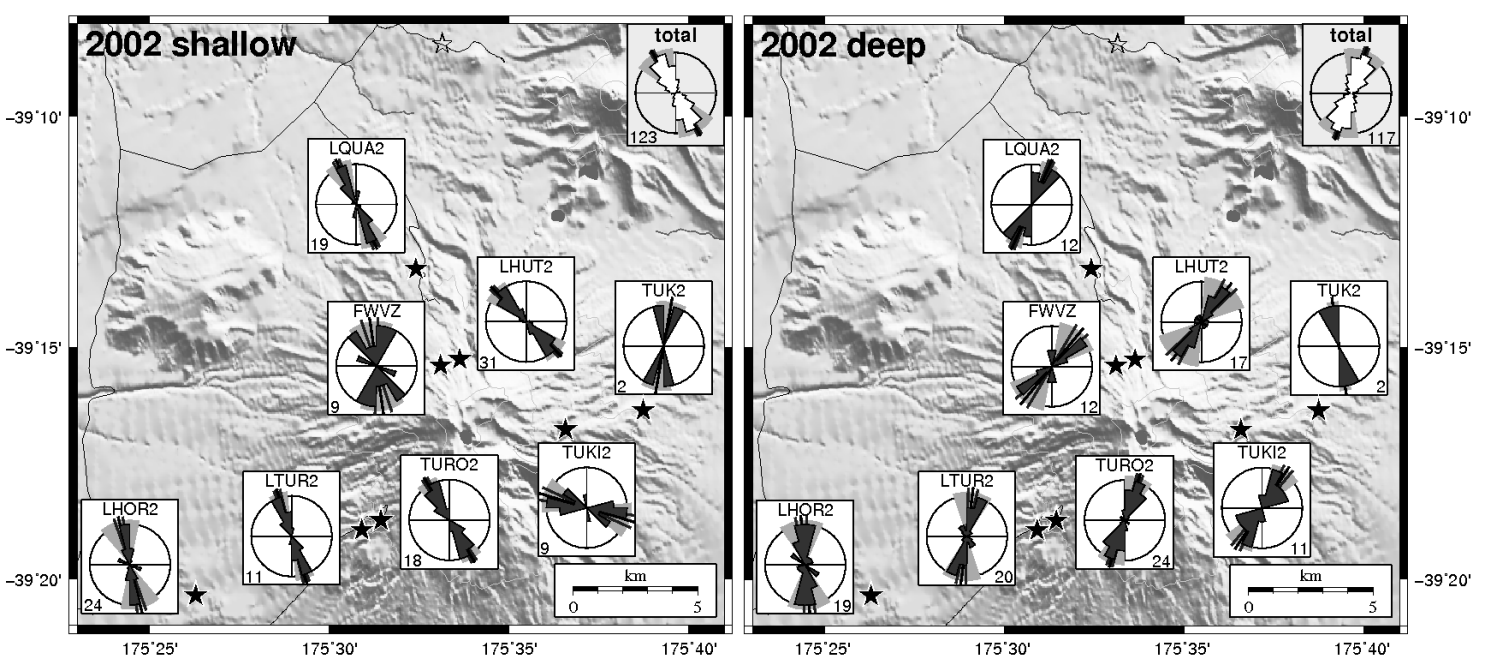

Figure 6.3.: Maps with histograms showing the distribution of fast orientations $\Phi$ for data from 2002 that were processed with a manual technique. The underlying grey area shows the standard deviation of the data. This figure is shown for comparison with Figure 6.4. (Source: Gerst and Savage, 2004)
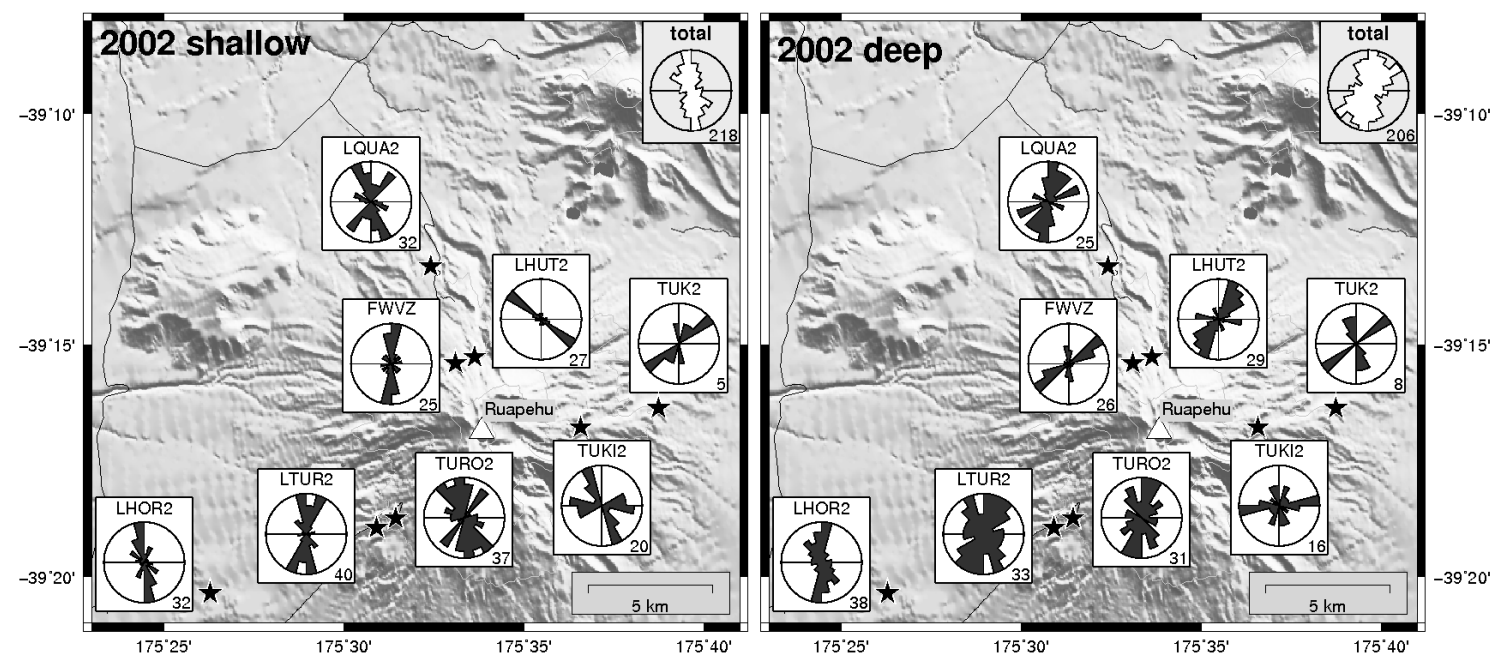

Figure 6.4.: Maps with histograms showing the distribution of fast orientations $\Phi$ for each station with data from 2002, calculated using the automatic technique on the entire 2002 data sets including noisy events that were subsequently rejected by the automatic technique. Up to three bandpass filters per event are used. Results from shallow $(0-30 \mathrm{~km})$ and deep $(\geq 55 \mathrm{~km})$ events are shown separately. See Table 6.2 for detailed numbers. 


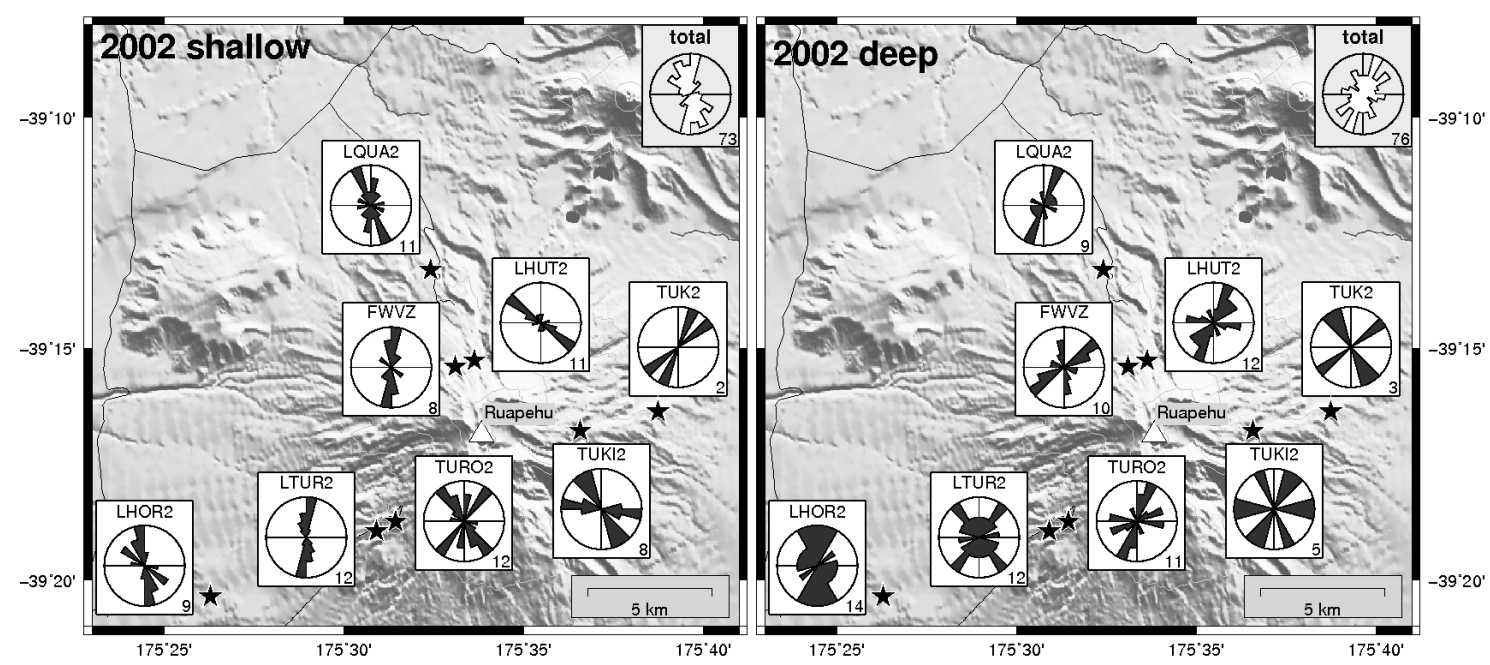

Figure 6.5.: Same as Figure 6.4, but only the best filter per event is considered. (The filter is chosen by a signal to noise ratio criterion. The filter that has the highest signal to noise ratio-measured in three-second windows before and after the S-pick-is considered as the best filter.) 


\subsubsection{New results from data collected since 2002}

Data recorded in 2003-2007 on numerous GeoNet stations have been processed with the new automatic shear wave splitting measurement technique. The results of the shear wave splitting measurements are shown in Table 6.3. Results from yearly data sets are listed for each station (sorted by distance from Crater Lake, close stations first) for single calendar years and for both shallow $(0-30 \mathrm{~km})$ and deep ( $\geq 55 \mathrm{~km})$ events. The separation of the data in shallow and deep events was adapted from Gerst and Savage (2004) and is also justified for the new data sets because of different mean fast directions for shallow and deep events at most stations.

Table 6.3.: Yearly results of individual stations. The results are sorted by distance of stations from Ruapehu first, then by shallow or deep data set and then by calendar year. Each entry contains the mean fast orientation of all measurements $\bar{\Phi}$ and its standard error $\pm \bar{\Phi}$, the mean delay time $\overline{\delta t}$ and the standard deviation of the delay times $\pm \delta t$, the mean resultant length $|\vec{R}|$ and the number of measurements \#. Data sets with at least 20 measurements and a mean resultant length $|\vec{R}| \geq 0.3$ are emphasised by bold font. A low value of $|\vec{R}|$ does not necessarily indicate a data set with erroneous measurements. It can also be caused by the presence of two different fast orientations in the same data set (e.g. deep events at station MOVZ, Figure 6.8, p. 111). Data from 2007 have only been processed up to July 2007. Earlier years have been considered once three-component data were available (see Chapter 5).

\begin{tabular}{|lll||r|r|r|r|r|r||}
\hline station & set & year & \multicolumn{1}{|c|}{$\Phi\left[^{\circ}\right]$} & $\pm \bar{\Phi}\left[^{\circ}\right]$ & $\overline{\delta t}[\mathrm{~s}]$ & $\pm \delta t[\mathrm{~s}]$ & $|\vec{R}|$ & $\#$ \\
\hline \hline TRVZ & shallow & $\mathbf{2 0 0 5}$ & $\mathbf{3 . 0}$ & $\mathbf{5 . 8}$ & $\mathbf{0 . 2 7}$ & $\mathbf{0 . 1 8}$ & $\mathbf{0 . 3 1}$ & $\mathbf{1 2 0}$ \\
TRVZ & shallow & $\mathbf{2 0 0 6}$ & $\mathbf{- 5 . 5}$ & $\mathbf{6 . 3}$ & $\mathbf{0 . 2 5}$ & $\mathbf{0 . 1 9}$ & $\mathbf{0 . 5 0}$ & $\mathbf{3 6}$ \\
TRVZ & shallow & 2007 & 1.5 & 5.3 & 0.18 & 0.12 & 0.84 & 10 \\
TRVZ & deep & 2005 & 4.9 & 16.3 & 0.30 & 0.18 & 0.18 & 48 \\
TRVZ & deep & 2006 & 26.5 & 12.1 & 0.24 & 0.14 & 0.47 & 11 \\
TRVZ & deep & 2007 & -5.7 & 14.5 & 0.35 & 0.07 & 0.56 & 5 \\
\hline FWVZ & shallow & 2003 & -11.0 & 6.9 & 0.24 & 0.17 & 0.17 & 314 \\
\hline
\end{tabular}


Table 6.3 - continued from previous page

\begin{tabular}{|c|c|c|c|c|c|c|c|c|}
\hline station & set & year & $\bar{\Phi}\left[^{\circ}\right]$ & $\pm \bar{\Phi}\left[{ }^{\circ}\right]$ & $\overline{\delta t}[\mathrm{~s}]$ & $\pm \delta t[\mathrm{~s}]$ & $|\vec{R}|$ & \# \\
\hline FWVZ & shallow & 2004 & -4.1 & 9.2 & 0.23 & 0.18 & 0.14 & 265 \\
\hline FWVZ & shallow & 2005 & 6.0 & 6.2 & 0.25 & 0.19 & 0.18 & 329 \\
\hline FWVZ & shallow & 2006 & -1.2 & 15.9 & 0.19 & 0.16 & 0.15 & 68 \\
\hline FWVZ & shallow & 2007 & -10.2 & 7.4 & 0.18 & 0.15 & 0.39 & 46 \\
\hline FWVZ & deep & 2003 & 68.9 & 14.4 & 0.30 & 0.17 & 0.15 & 92 \\
\hline FWVZ & deep & 2004 & 50.7 & 10.8 & 0.30 & 0.17 & 0.17 & 114 \\
\hline FWVZ & deep & 2005 & 36.5 & 8.1 & 0.27 & 0.17 & 0.21 & 140 \\
\hline FWVZ & deep & 2006 & 36.1 & 15.8 & 0.22 & 0.14 & 0.23 & 29 \\
\hline FWVZ & deep & 2007 & -77.9 & 21.6 & 0.26 & 0.11 & 0.23 & 16 \\
\hline WNVZ & shallow & 2004 & 8.1 & 7.0 & 0.30 & 0.20 & 0.22 & 169 \\
\hline WNVZ & shallow & 2005 & -0.6 & 3.9 & 0.28 & 0.17 & 0.30 & 276 \\
\hline WNVZ & shallow & 2006 & 0.4 & 5.9 & 0.20 & 0.12 & 0.47 & 46 \\
\hline WNVZ & shallow & 2007 & -16.7 & 20.0 & 0.20 & 0.15 & 0.24 & 17 \\
\hline WNVZ & deep & 2004 & -1.1 & 6.1 & 0.25 & 0.17 & 0.33 & 99 \\
\hline WNVZ & deep & 2005 & -3.8 & 4.0 & 0.26 & 0.16 & 0.33 & 228 \\
\hline WNVZ & deep & 2006 & -2.5 & 4.9 & 0.24 & 0.16 & 0.57 & 43 \\
\hline WNVZ & deep & 2007 & -25.7 & 41.3 & 0.34 & 0.21 & 0.13 & 14 \\
\hline TUVZ & shallow & 2004 & 31.6 & 9.6 & 0.32 & 0.20 & 0.19 & 122 \\
\hline TUVZ & shallow & 2005 & 31.9 & 3.7 & 0.30 & 0.18 & 0.33 & 256 \\
\hline TUVZ & shallow & 2006 & 42.0 & 8.1 & 0.30 & 0.21 & 0.39 & 39 \\
\hline TUVZ & shallow & 2007 & 37.0 & 11.5 & 0.31 & 0.20 & 0.46 & 13 \\
\hline TUVZ & deep & 2004 & 36.6 & 4.4 & 0.32 & 0.16 & 0.50 & 73 \\
\hline TUVZ & deep & 2005 & 40.3 & 3.6 & 0.34 & 0.17 & 0.43 & 157 \\
\hline TUVZ & deep & 2006 & 34.9 & 4.6 & 0.32 & 0.16 & 0.65 & 34 \\
\hline
\end{tabular}


Table 6.3 - continued from previous page

\begin{tabular}{|c|c|c|c|c|c|c|c|c|}
\hline station & set & year & $\bar{\Phi}\left[^{\circ}\right]$ & $\pm \bar{\Phi}\left[{ }^{\circ}\right]$ & $\overline{\delta t}[\mathrm{~s}]$ & $\pm \delta t[\mathrm{~s}]$ & $|\vec{R}|$ & $\#$ \\
\hline TUVZ & deep & 2007 & 34.9 & 20.0 & 0.34 & 0.19 & 0.27 & 14 \\
\hline WPVZ & shallow & 2004 & -15.0 & 6.2 & 0.22 & 0.18 & 0.20 & 261 \\
\hline WPVZ & shallow & 2005 & -26.5 & 5.3 & 0.19 & 0.15 & 0.23 & 276 \\
\hline WPVZ & shallow & 2006 & -31.6 & 8.5 & 0.20 & 0.13 & 0.29 & 66 \\
\hline WPVZ & shallow & 2007 & 4.8 & 17.0 & 0.17 & 0.18 & 0.26 & 21 \\
\hline WPVZ & deep & 2004 & -17.7 & 22.5 & 0.26 & 0.15 & 0.11 & 69 \\
\hline WPVZ & deep & 2005 & -17.6 & 11.8 & 0.24 & 0.14 & 0.17 & 105 \\
\hline WPVZ & deep & 2006 & -64.9 & 18.4 & 0.20 & 0.17 & 0.27 & 16 \\
\hline WPVZ & deep & 2007 & -0.0 & 27.2 & 0.23 & 0.18 & 0.24 & 9 \\
\hline NGZ & shallow & 2004 & -39.4 & 13.9 & 0.28 & 0.16 & 0.12 & 136 \\
\hline NGZ & shallow & 2005 & -52.5 & 29.7 & 0.33 & 0.18 & 0.06 & 134 \\
\hline NGZ & shallow & 2006 & -56.1 & 42.7 & 0.36 & 0.20 & 0.10 & 25 \\
\hline NGZ & shallow & 2007 & -35.3 & 18.1 & 0.36 & 0.21 & 0.47 & 5 \\
\hline NGZ & deep & 2004 & 39.1 & 10.9 & 0.38 & 0.18 & 0.24 & 60 \\
\hline NGZ & deep & 2005 & 32.4 & 8.2 & 0.39 & 0.22 & 0.28 & 76 \\
\hline NGZ & deep & 2006 & 30.7 & 16.1 & 0.33 & 0.16 & 0.31 & 16 \\
\hline MTVZ & shallow & 2005 & 88.1 & 10.6 & 0.29 & 0.20 & 0.14 & 194 \\
\hline MTVZ & shallow & 2006 & 45.2 & 15.3 & 0.33 & 0.22 & 0.17 & 57 \\
\hline MTVZ & shallow & 2007 & 74.1 & 20.5 & 0.32 & 0.20 & 0.17 & 32 \\
\hline MTVZ & deep & 2005 & 7.9 & 12.8 & 0.27 & 0.18 & 0.14 & 121 \\
\hline MTVZ & deep & 2006 & -1.9 & 11.3 & 0.23 & 0.17 & 0.27 & 44 \\
\hline MTVZ & deep & 2007 & -1.2 & 10.4 & 0.27 & 0.17 & 0.39 & 23 \\
\hline OTVZ & shallow & 2004 & -89.9 & 14.3 & 0.20 & 0.13 & 0.33 & 17 \\
\hline OTVZ & shallow & 2005 & -82.9 & 5.1 & 0.23 & 0.16 & 0.30 & 163 \\
\hline
\end{tabular}


Table 6.3 - continued from previous page

\begin{tabular}{|c|c|c|c|c|c|c|c|c|}
\hline station & set & year & $\bar{\Phi}\left[^{\circ}\right]$ & $\pm \bar{\Phi}\left[{ }^{\circ}\right]$ & $\overline{\delta t}[\mathrm{~s}]$ & $\pm \delta t[\mathrm{~s}]$ & $|\vec{R}|$ & $\#$ \\
\hline OTVZ & shallow & 2006 & -74.1 & 10.7 & 0.37 & 0.20 & 0.31 & 36 \\
\hline OTVZ & shallow & 2007 & -86.4 & 19.3 & 0.22 & 0.16 & 0.31 & 11 \\
\hline OTVZ & deep & 2004 & 82.5 & 13.4 & 0.23 & 0.12 & 0.60 & 5 \\
\hline OTVZ & deep & 2005 & 86.4 & 4.4 & 0.28 & 0.17 & 0.44 & 100 \\
\hline OTVZ & deep & 2006 & 76.4 & 5.3 & 0.27 & 0.20 & 0.54 & 42 \\
\hline OTVZ & deep & 2007 & 71.9 & 12.7 & 0.24 & 0.14 & 0.47 & 10 \\
\hline WTVZ & shallow & 2004 & 73.8 & 8.2 & 0.25 & 0.18 & 0.18 & 190 \\
\hline WTVZ & shallow & 2005 & 79.6 & 6.3 & 0.28 & 0.20 & 0.21 & 238 \\
\hline WTVZ & shallow & 2006 & 62.5 & 15.5 & 0.23 & 0.20 & 0.20 & 44 \\
\hline WTVZ & shallow & 2007 & -57.4 & 43.5 & 0.19 & 0.17 & 0.12 & 14 \\
\hline WTVZ & deep & 2004 & 6.9 & 7.6 & 0.33 & 0.17 & 0.34 & 59 \\
\hline WTVZ & deep & 2005 & 6.7 & 8.0 & 0.33 & 0.18 & 0.29 & 74 \\
\hline WTVZ & deep & 2006 & -9.2 & 8.5 & 0.36 & 0.20 & 0.41 & 30 \\
\hline WTVZ & deep & 2007 & -0.5 & 47.1 & 0.36 & 0.24 & 0.16 & 7 \\
\hline PKVZ & shallow & 2005 & 54.4 & 6.7 & 0.25 & 0.18 & 0.29 & 102 \\
\hline PKVZ & shallow & 2006 & 61.9 & 7.3 & 0.22 & 0.18 & 0.39 & 46 \\
\hline PKVZ & shallow & 2007 & 38.8 & 17.4 & 0.31 & 0.18 & 0.23 & 25 \\
\hline PKVZ & deep & 2005 & 57.4 & 9.3 & 0.24 & 0.17 & 0.24 & 78 \\
\hline PKVZ & deep & 2006 & 33.9 & 4.9 & 0.17 & 0.13 & 0.67 & 28 \\
\hline PKVZ & deep & 2007 & 37.4 & 16.7 & 0.23 & 0.15 & 0.39 & 9 \\
\hline MOVZ & shallow & 2004 & 5.7 & 15.2 & 0.24 & 0.16 & 0.10 & 176 \\
\hline MOVZ & shallow & 2005 & 23.8 & 6.9 & 0.25 & 0.15 & 0.16 & 343 \\
\hline MOVZ & shallow & 2006 & 0.2 & 6.4 & 0.29 & 0.16 & 0.33 & 87 \\
\hline MOVZ & shallow & 2007 & -19.9 & 12.9 & 0.25 & 0.12 & 0.26 & 35 \\
\hline
\end{tabular}


Table 6.3 - continued from previous page

\begin{tabular}{|lll||r|r|r|r|r|r||}
\hline station & set & year & \multicolumn{1}{c|}{$\overline{\left.{ }^{\circ}\right]}$} & $\pm \bar{\Phi}\left[^{\circ}\right]$ & $\overline{\delta t}[\mathrm{~s}]$ & $\pm \delta t[\mathrm{~s}]$ & $|\vec{R}|$ & $\#$ \\
\hline \hline MOVZ & deep & $\mathbf{2 0 0 4}$ & $\mathbf{- 3 . 7}$ & $\mathbf{2 . 5}$ & $\mathbf{0 . 2 0}$ & $\mathbf{0 . 1 8}$ & $\mathbf{0 . 6 6}$ & $\mathbf{1 1 4}$ \\
MOVZ & deep & $\mathbf{2 0 0 5}$ & $\mathbf{3 . 8}$ & $\mathbf{1 . 8}$ & $\mathbf{0 . 2 5}$ & $\mathbf{0 . 2 1}$ & $\mathbf{0 . 6 5}$ & $\mathbf{2 3 4}$ \\
MOVZ & deep & $\mathbf{2 0 0 6}$ & $\mathbf{1 . 7}$ & $\mathbf{2 . 6}$ & $\mathbf{0 . 2 4}$ & $\mathbf{0 . 2 0}$ & $\mathbf{0 . 6 6}$ & $\mathbf{9 8}$ \\
MOVZ & deep & $\mathbf{2 0 0 7}$ & $\mathbf{1 1 . 6}$ & $\mathbf{3 . 8}$ & $\mathbf{0 . 2 5}$ & $\mathbf{0 . 1 8}$ & $\mathbf{0 . 7 9}$ & $\mathbf{2 5}$ \\
\hline TWVZ & shallow & 2004 & -81.2 & 7.6 & 0.22 & 0.20 & 0.28 & 87 \\
TWVZ & shallow & $\mathbf{2 0 0 5}$ & $\mathbf{- 8 8 . 1}$ & $\mathbf{5 . 9}$ & $\mathbf{0 . 1 9}$ & $\mathbf{0 . 1 6}$ & $\mathbf{0 . 3 2}$ & $\mathbf{1 1 1}$ \\
TWVZ & shallow & $\mathbf{2 0 0 6}$ & $\mathbf{- 7 4 . 8}$ & $\mathbf{9 . 9}$ & $\mathbf{0 . 1 8}$ & $\mathbf{0 . 1 5}$ & $\mathbf{0 . 3 7}$ & $\mathbf{2 9}$ \\
TWVZ & shallow & 2007 & -25.1 & 13.8 & 0.12 & 0.05 & 0.34 & 17 \\
TWVZ & deep & $\mathbf{2 0 0 4}$ & $\mathbf{1 . 9}$ & $\mathbf{9 . 8}$ & $\mathbf{0 . 2 7}$ & $\mathbf{0 . 1 7}$ & $\mathbf{0 . 3 2}$ & $\mathbf{4 0}$ \\
TWVZ & deep & $\mathbf{2 0 0 5}$ & $\mathbf{- 6 . 5}$ & $\mathbf{7 . 1}$ & $\mathbf{0 . 2 7}$ & $\mathbf{0 . 1 7}$ & $\mathbf{0 . 3 3}$ & $\mathbf{7 1}$ \\
TWVZ & deep & $\mathbf{2 0 0 6}$ & $\mathbf{1 . 0}$ & $\mathbf{7 . 4}$ & $\mathbf{0 . 2 5}$ & $\mathbf{0 . 1 3}$ & $\mathbf{0 . 4 8}$ & $\mathbf{2 9}$ \\
TWVZ & deep & 2007 & -12.0 & 10.7 & 0.32 & 0.14 & 0.57 & 9 \\
\hline \hline
\end{tabular}

Maps with rose diagrams illustrating the distribution of the fast orientation of yearly data sets (2004-2007) are shown below (Figures 6.6-6.10). Only two permanent three-component GeoNet stations were operating within $30 \mathrm{~km}$ of Mt. Ruapehu in 2003 (Figure 5.2, p. 95). One station (DRZ) is located close to the summit and often recorded noisy data without clear S-wave onsets. Its data has been ignored for the automatic measurements.

The results will be discussed in the next chapter. Maps with results of single measurements (split-maps) are shown in Appendix C, $163 \mathrm{ff}$. 


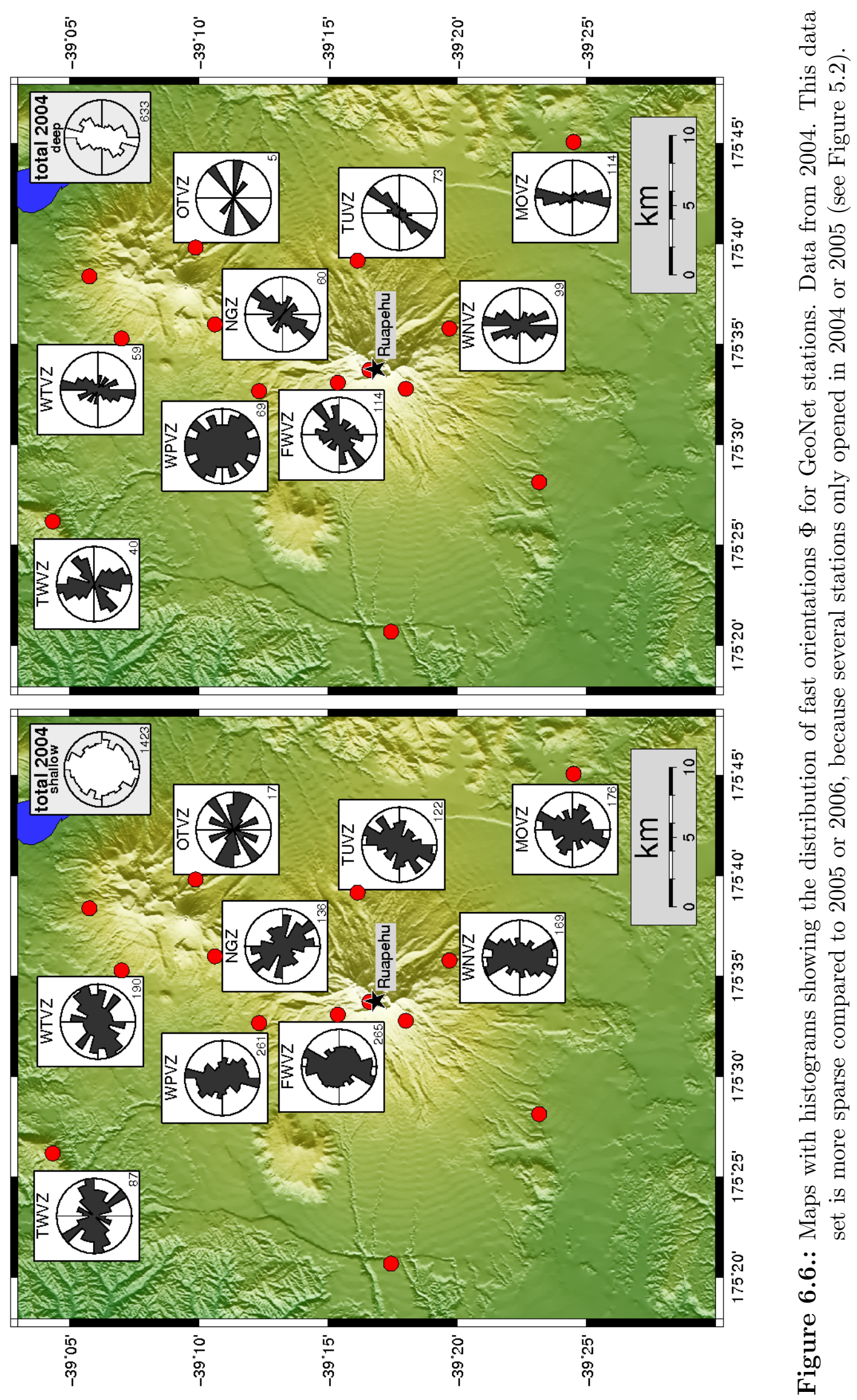




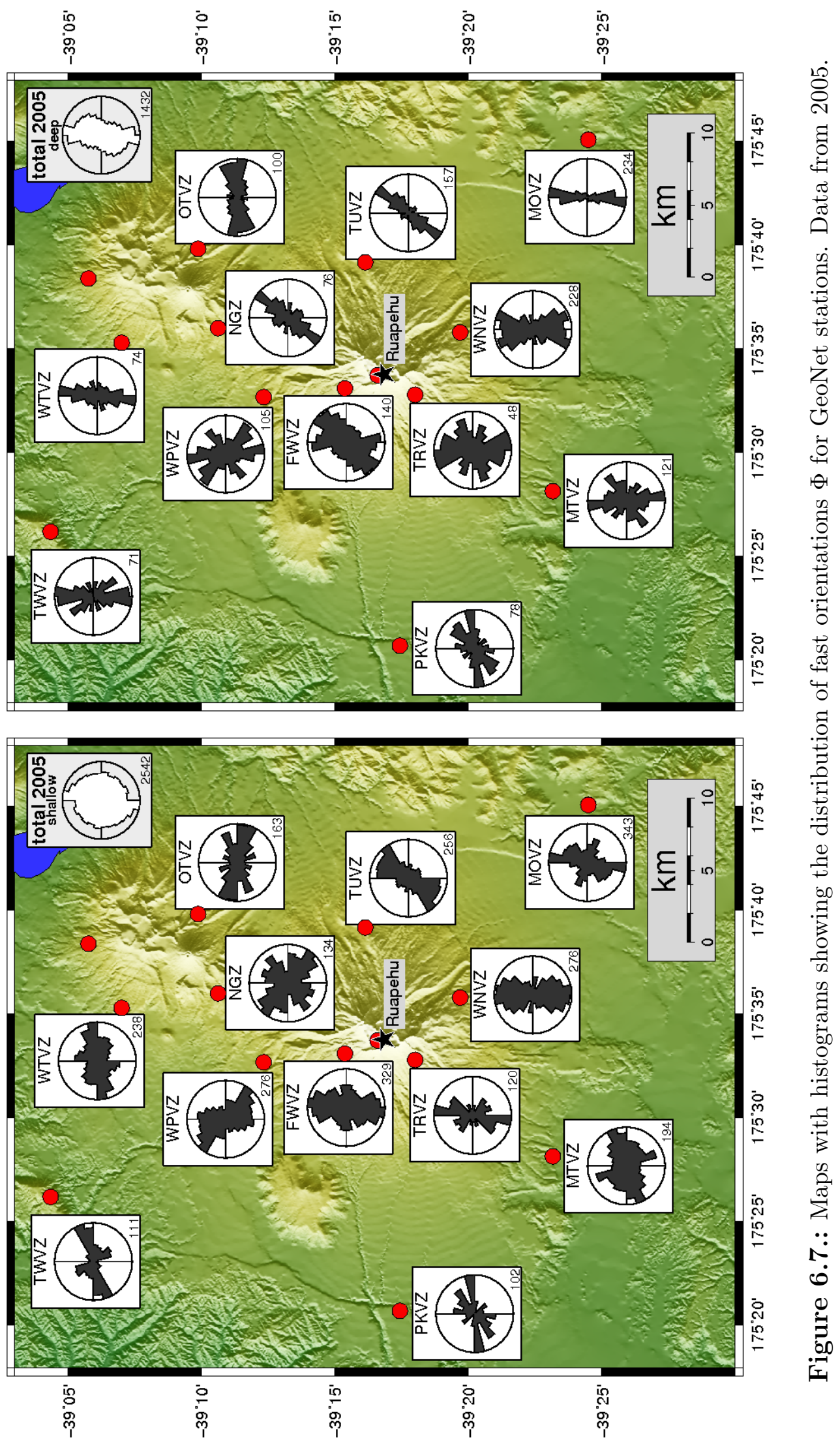




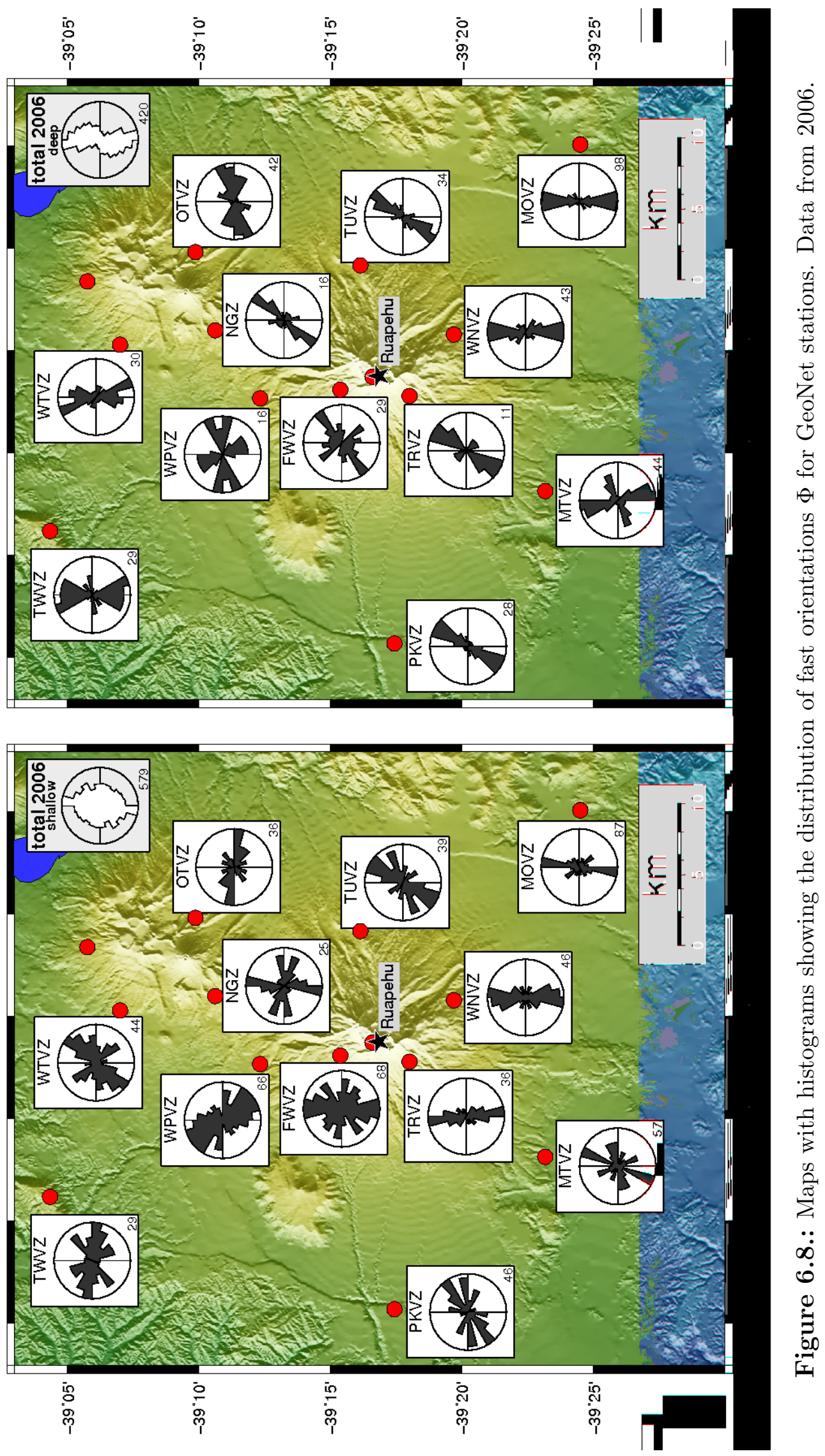




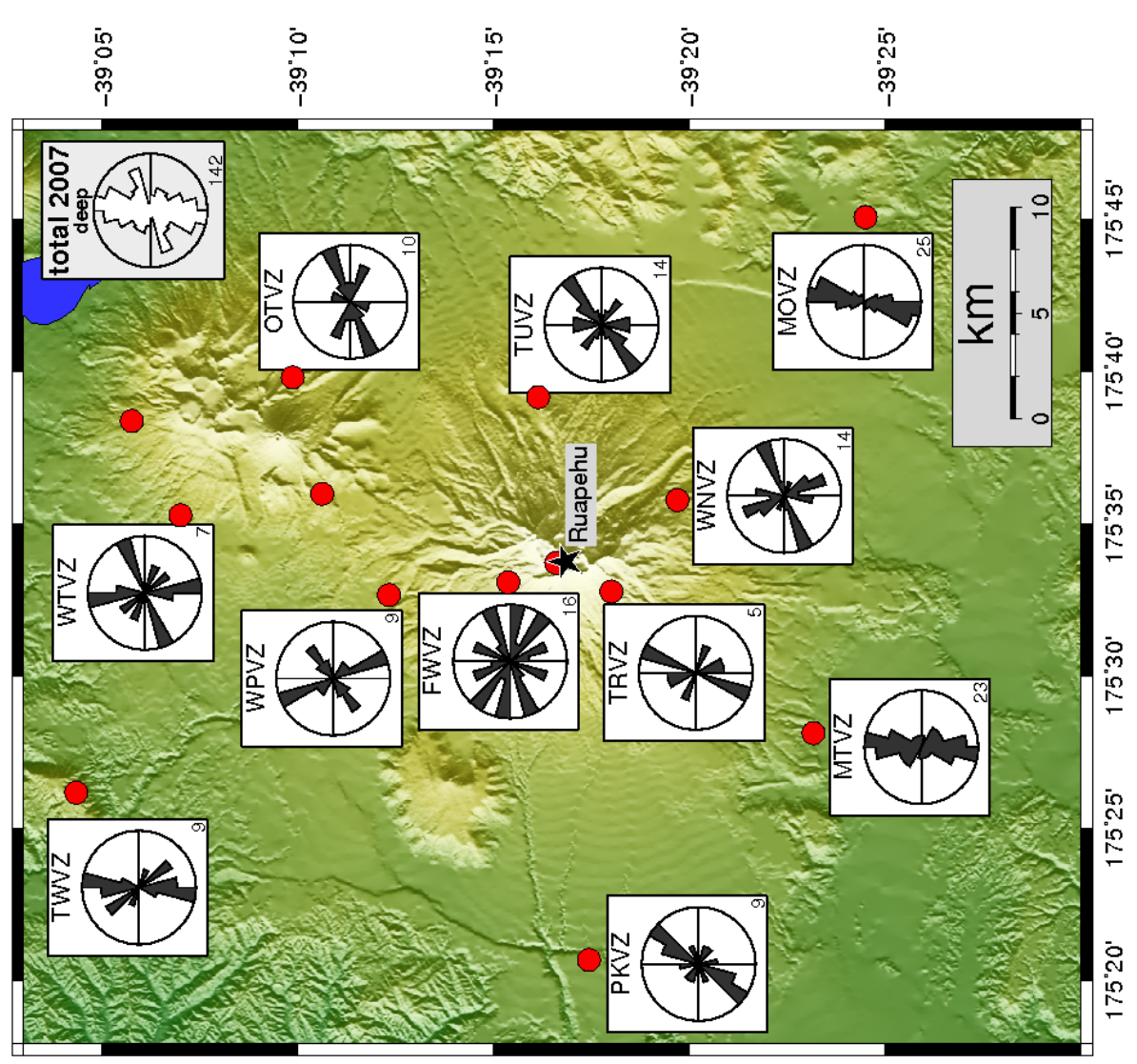

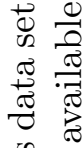

$\because$

迹

$\frac{11}{11}$

3

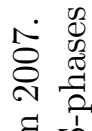

घี के

$\stackrel{0}{*}$

营政

ค.

m

.용

西

苍主

8

ن

ar

궁

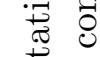

름

(n)

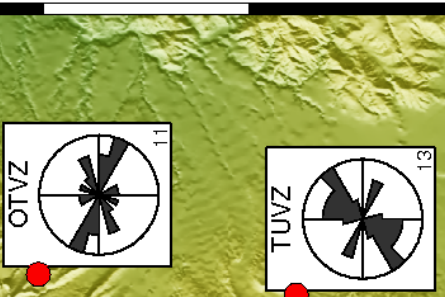

as

满

要

is

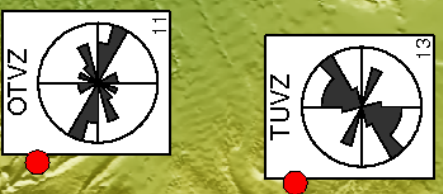

$\frac{E_{1}^{+}}{I_{0}^{\circ}}$

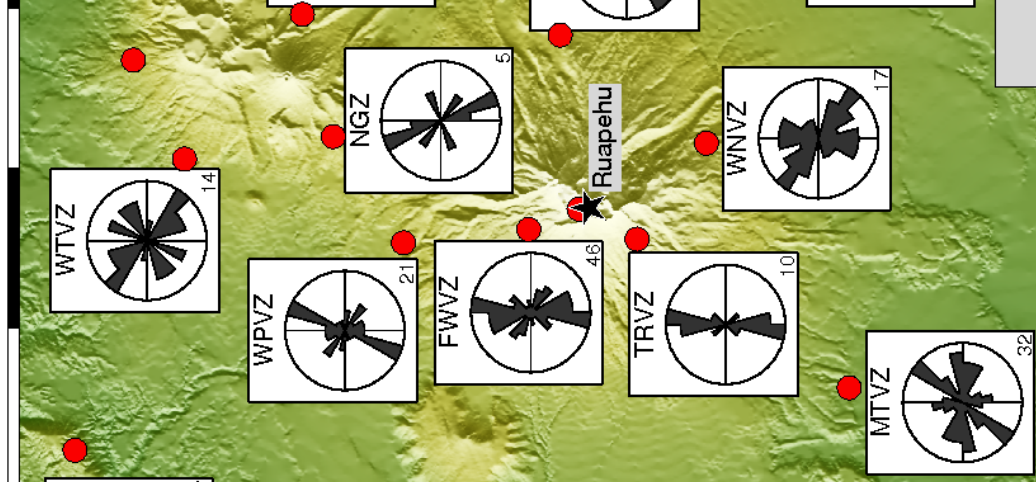

$N^{2}$

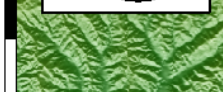
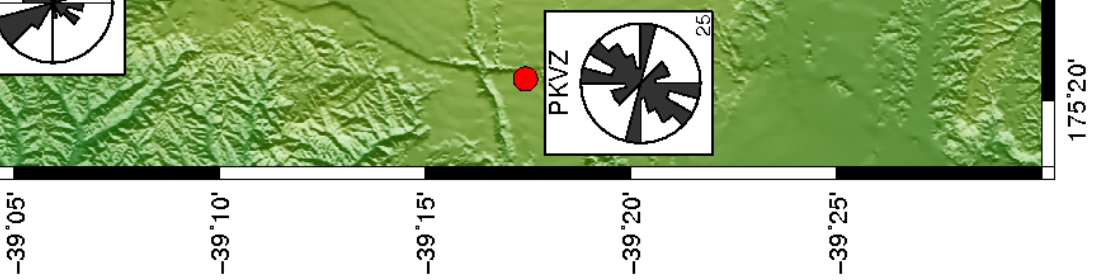

.ำ

\%

चै की

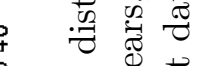

in $\quad 0 \stackrel{0}{1}$

ڤె

.

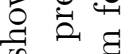

a

ส

范范

总范范

疍

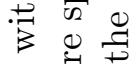

क्?

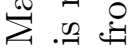

$\ddot{0}$

: 


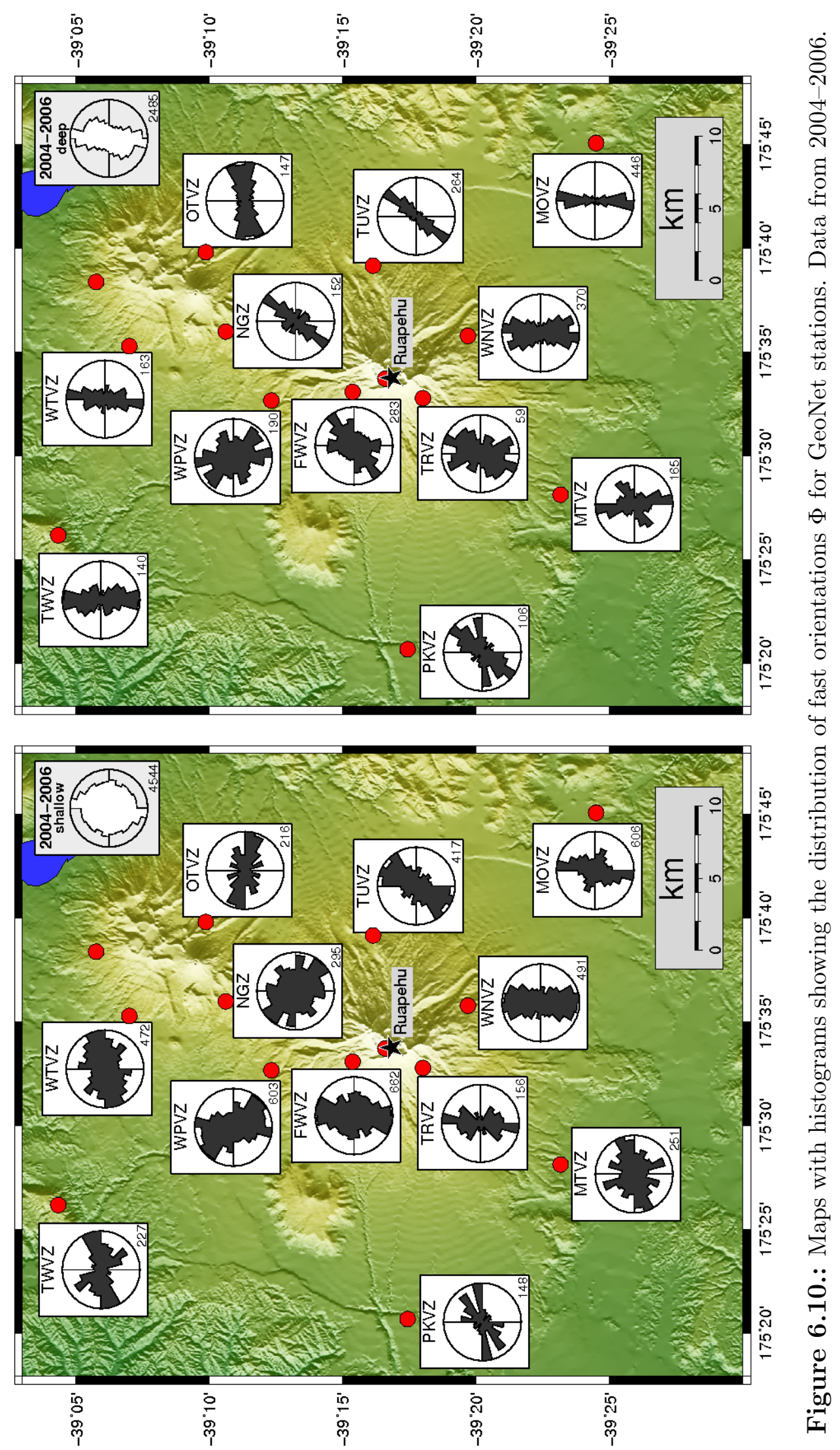


To investigate temporal changes, the shear wave splitting parameters are plotted against time for each station. To account for the large scatter of single measurements, a moving-window average is calculated to "smooth" the data and show long-period temporal trends. To calculate the moving-window average I used the script window_ avg.py* and modified the calculation of mean values for orientation data to account for the $180^{\circ}$-ambiguity of orientation data (see Section 4.3.4). The fast orientation $\Phi$ is shown from -90 to $90^{\circ}$, except for some plots where the values were close to the edge and the scale was shifted to avoid "skipping" of values. ${ }^{\dagger}$

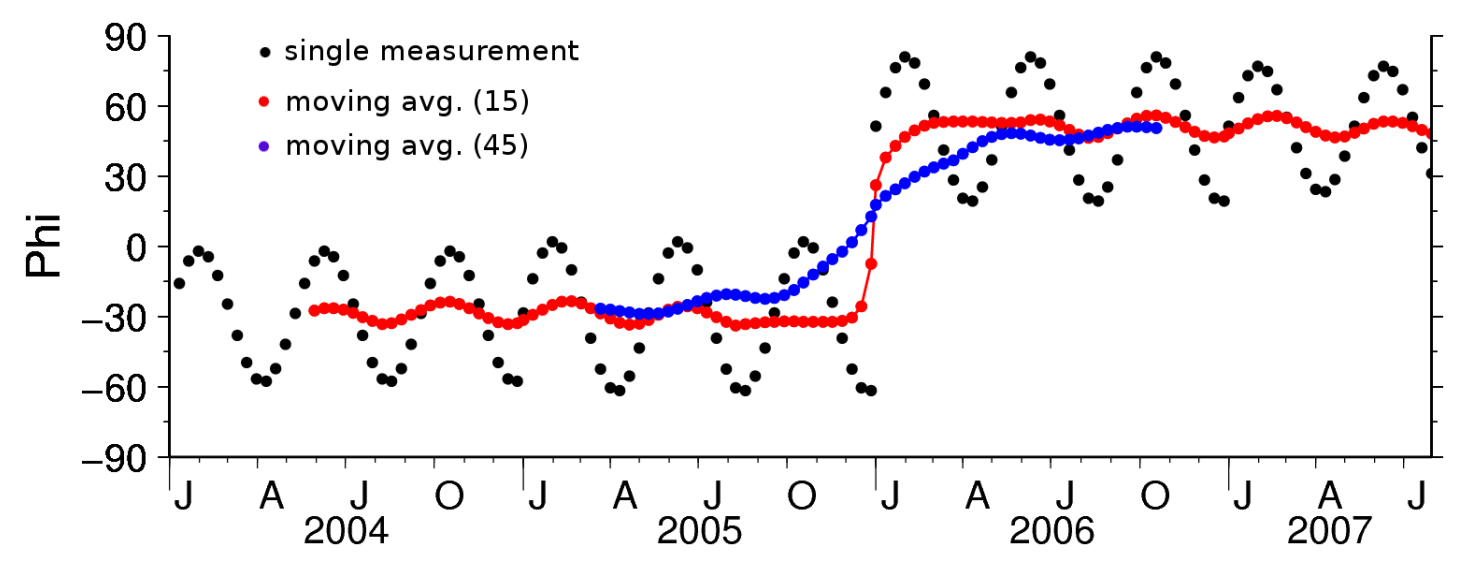

Figure 6.11.: Example of moving-window averaged data (synthetic data). Data that are scattered (here represented by a sinusoidal pattern) around mean values of $-30^{\circ}$ in $2004 / 2005$ and around $50^{\circ}$ in $2006 / 2007$ are shown as single measurements (with one datum every ten days) and after the calculation of moving-window averages with half-width 15 (30-point window) and half-width 45 (90-point window). These example data are chosen to show how a hypothetical temporal change of $80^{\circ}$ in the mean fast orientation between 2005 and 2006 would be displayed.

The following figures show moving-window averaged shear wave splitting parameters (delay time and fast orientation) for several years of data from each station. Figures are sorted by their distance from Mt. Ruapehu (close stations first). Red points in these plots denote values of a 30-point averaging window, blue dots mark values of a 90-point averaging window. The results will be discussed in Section 7.3.

*Original script by Robert Campbell, http://pldserver1.biochem.queensu.ca/ rlc/ work/scripts/, visited 1 November 2007. Used with permission.

${ }^{\dagger}$ Two values of -91 and 91 would plot on opposite ends of the diagram, but due to the $180^{\circ}$ ambiguity of orientation data these values only differ by two degrees $\left(-91^{\circ}\right.$ equals $\left.89^{\circ}\right)$. 


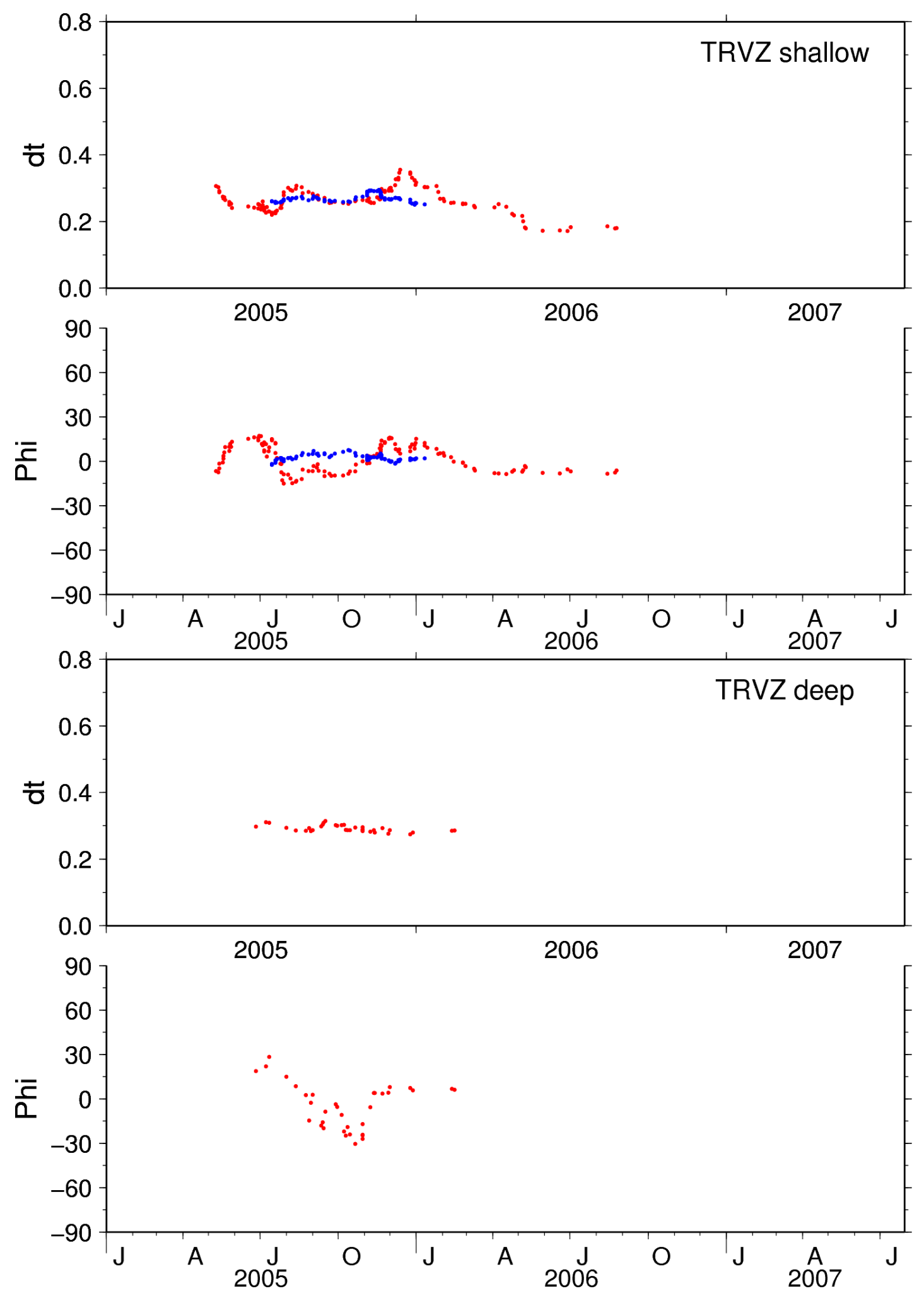

Figure 6.12.: Moving-window averaged results (delay time and fast orientation) from station TRVZ for shallow (top two plots) and deep events (lower two plots). Red dots denote values of a 30-point window, blue dots mark values of a 90 -point window (see text). 


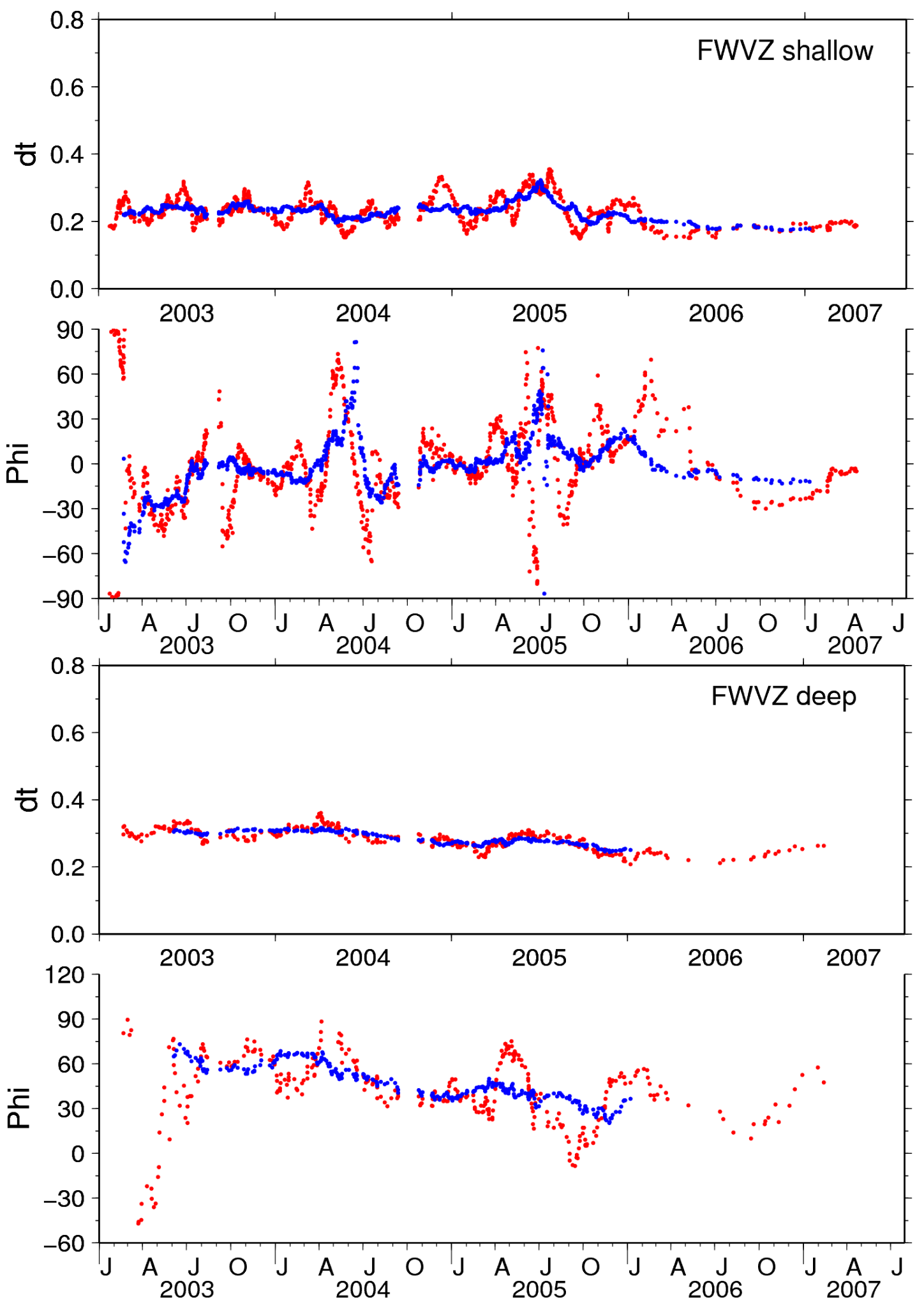

Figure 6.13.: Moving-window averaged results from station FWVZ. The scale has been shifted for deep events. 


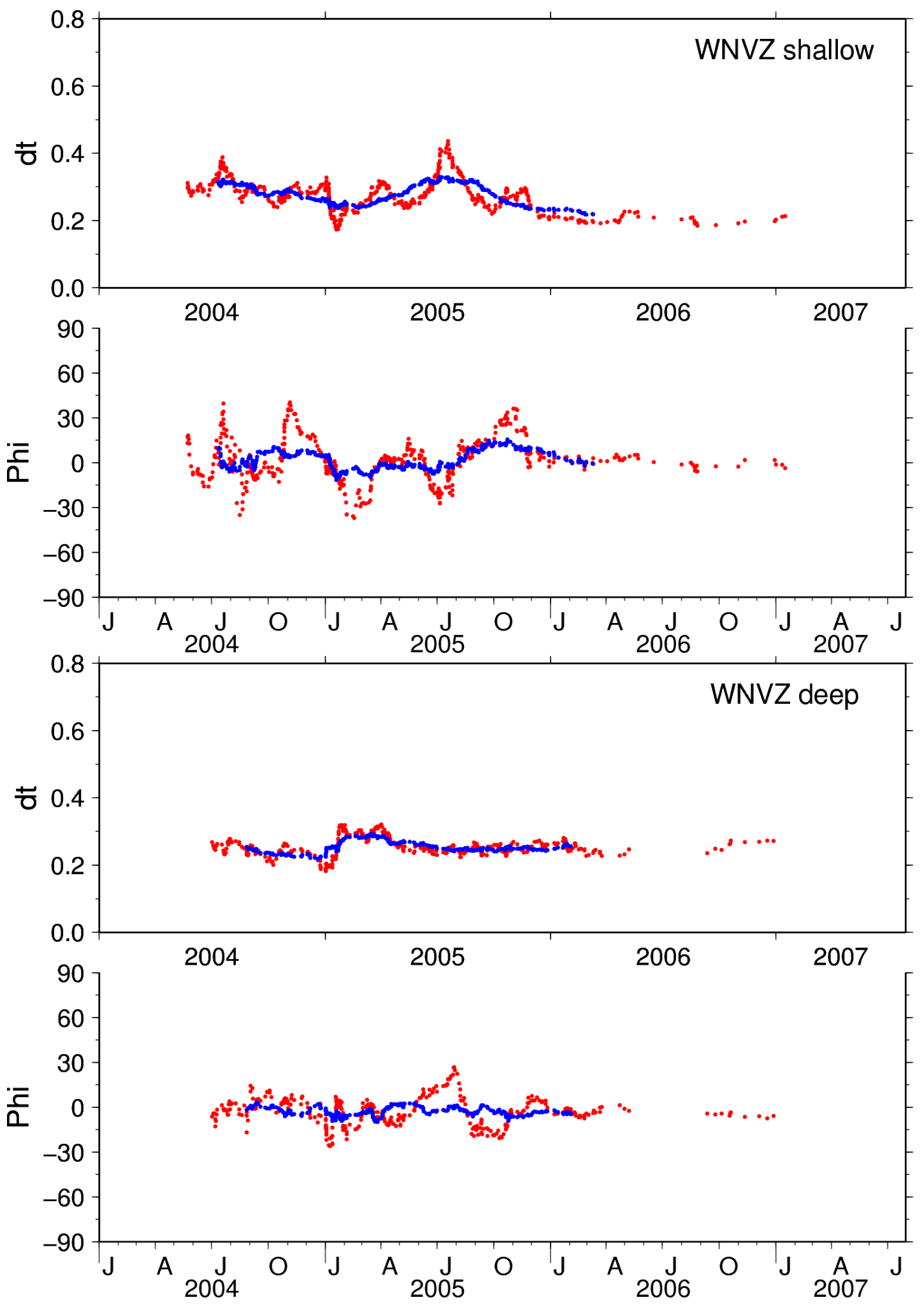

Figure 6.14.: Moving-window averaged results from station WNVZ. 


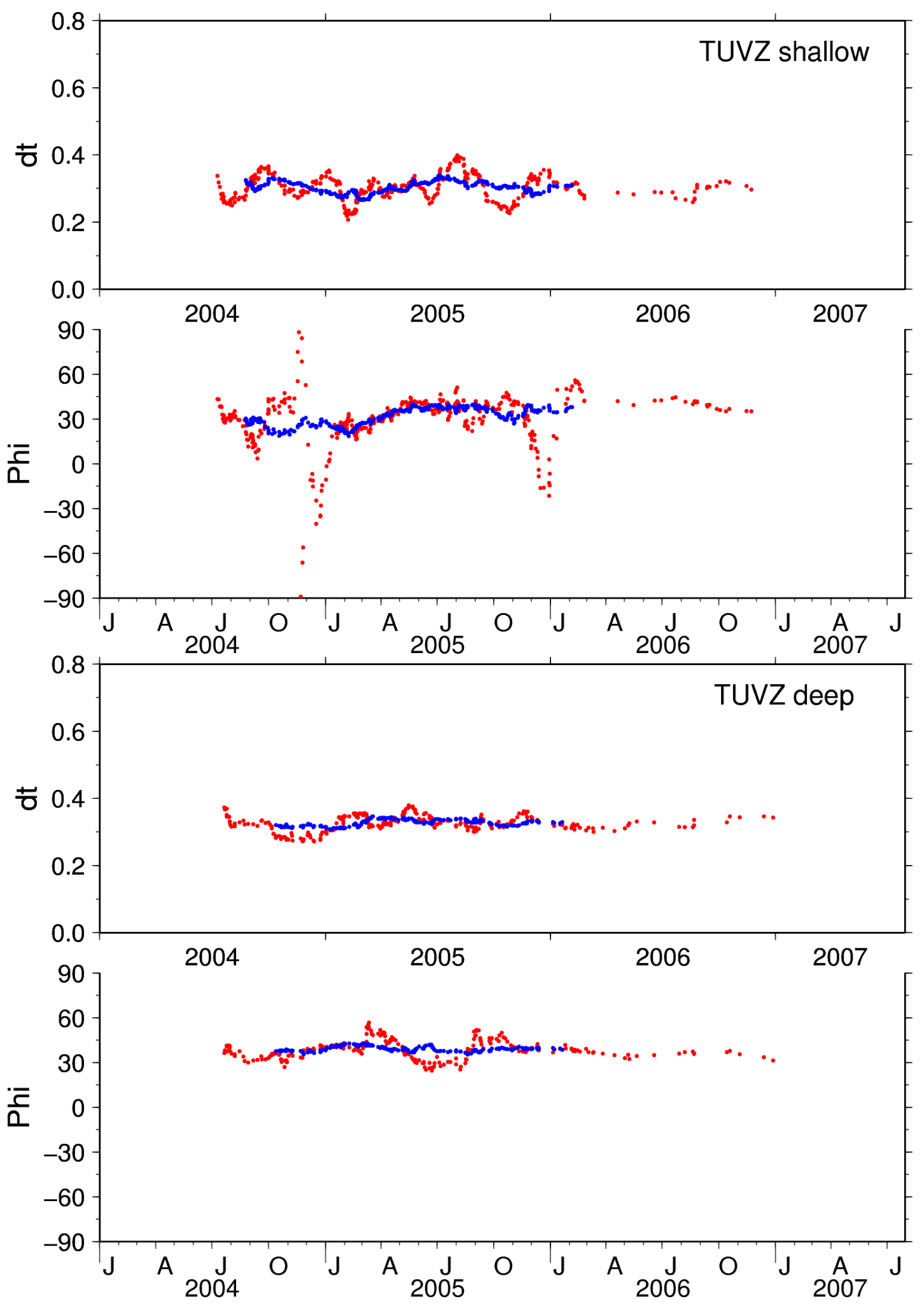

Figure 6.15.: Moving-window averaged results from station TUVZ. 


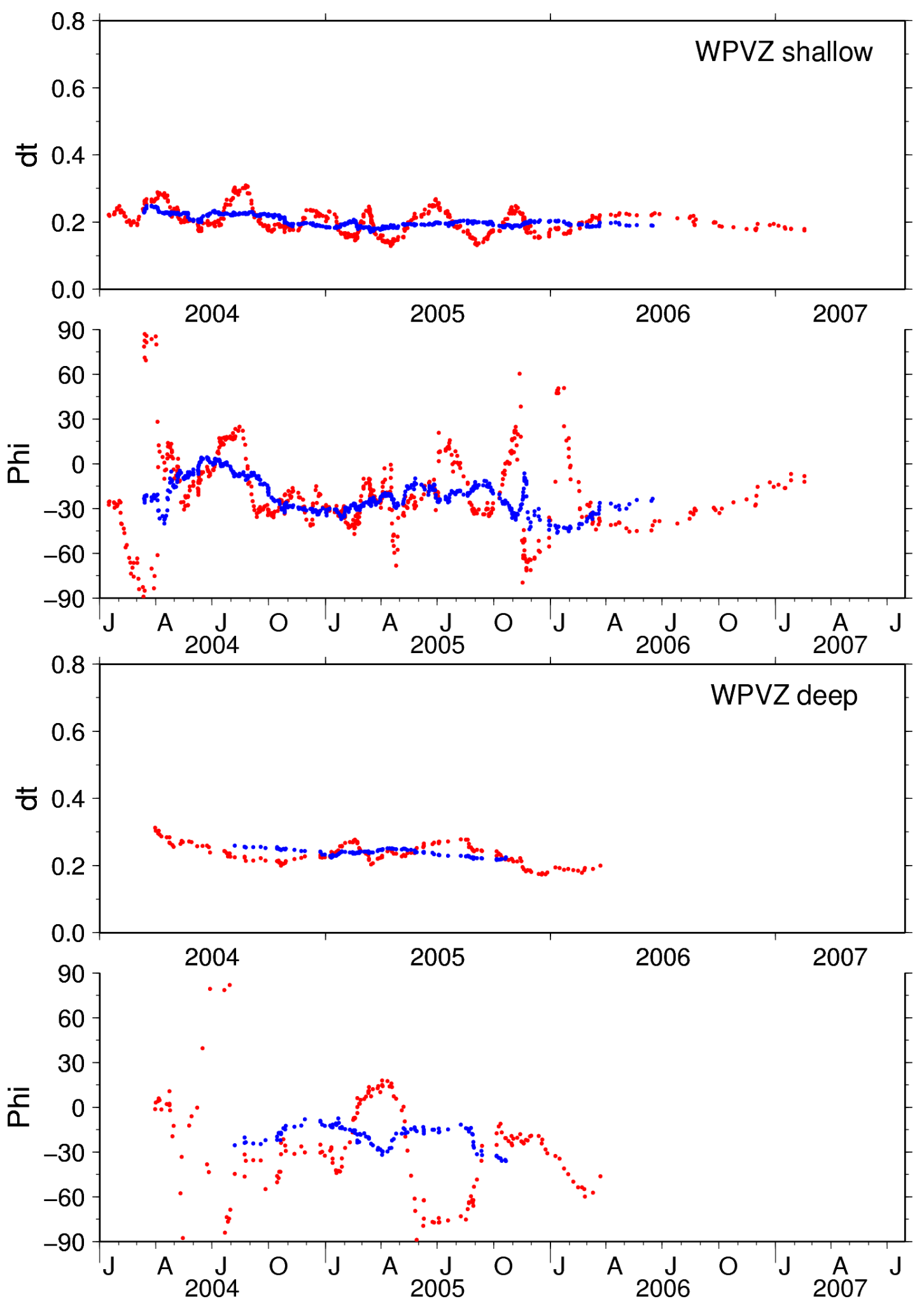

Figure 6.16.: Moving-window averaged results from station WPVZ. 


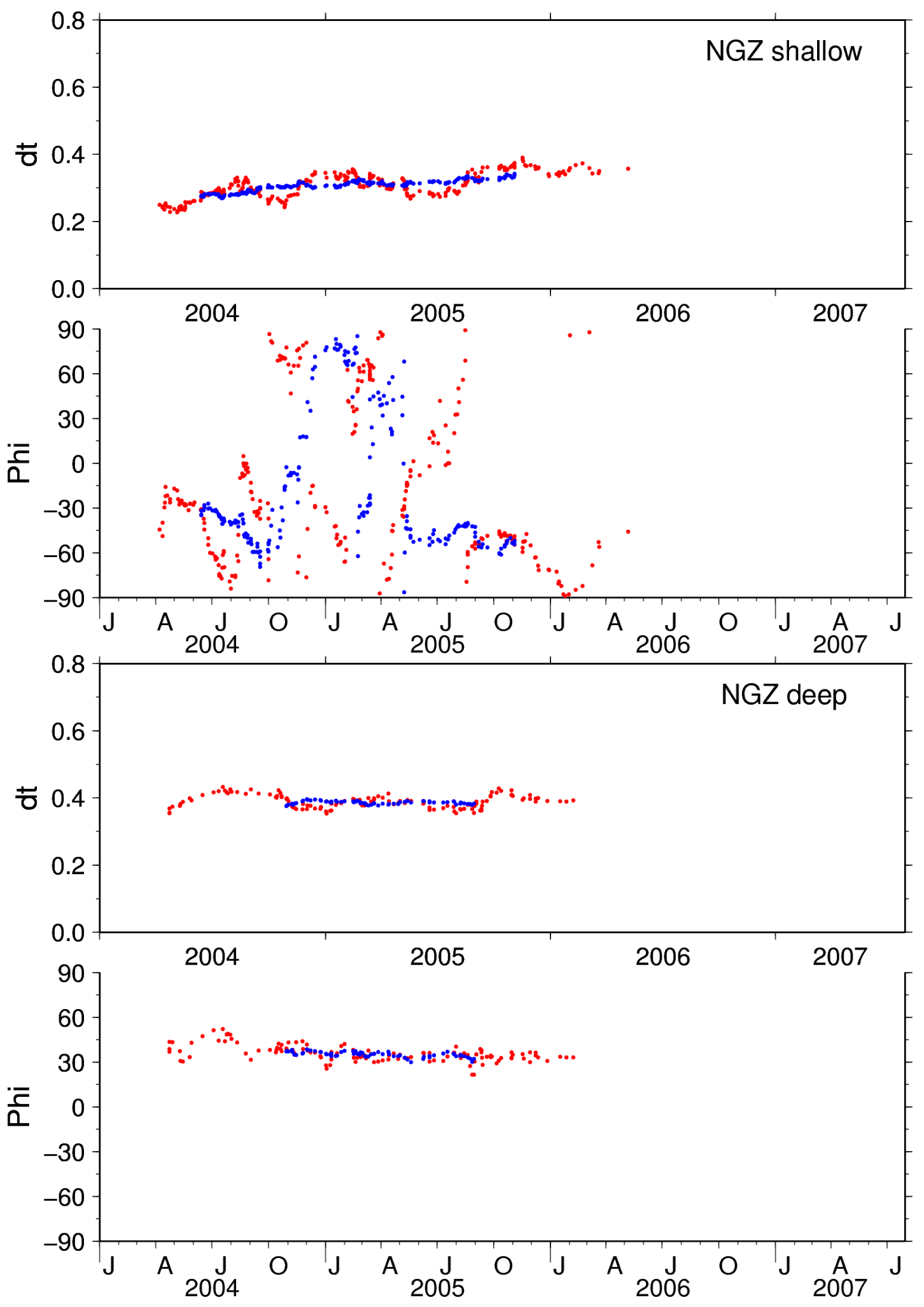

Figure 6.17.: Moving-window averaged results from station NGZ. 

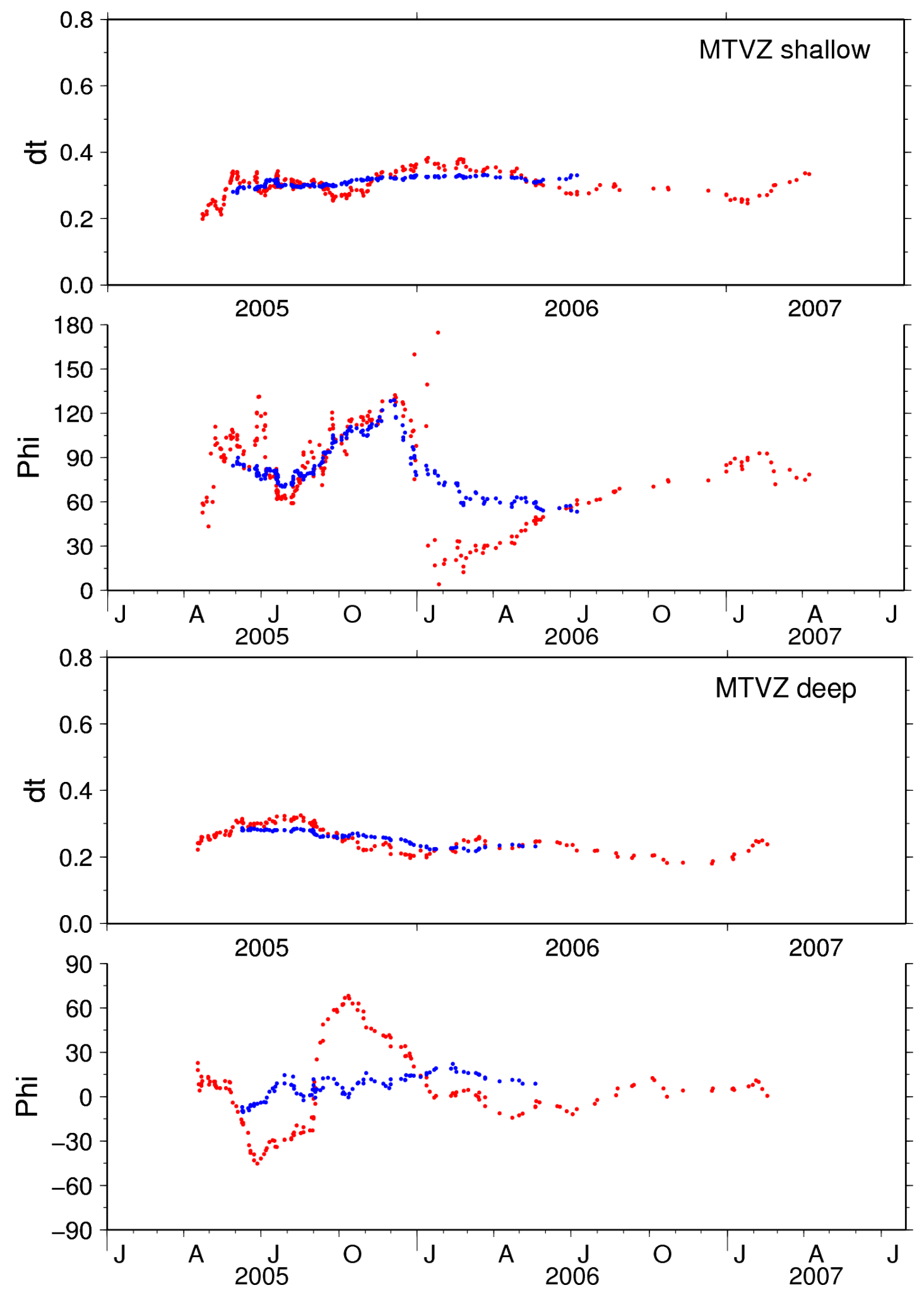

Figure 6.18.: Moving-window averaged results from station MTVZ. The scale has been shifted for shallow events. 


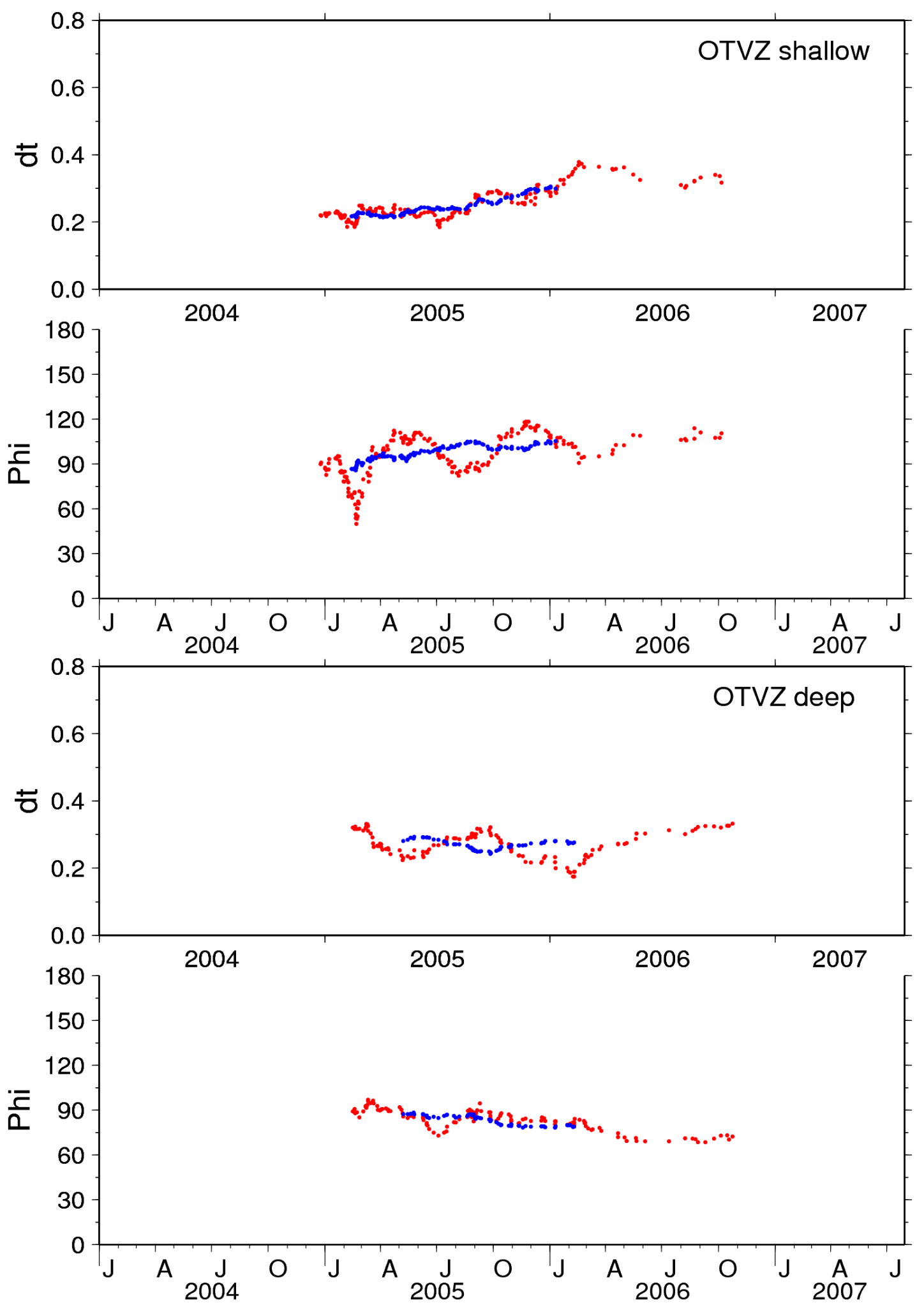

Figure 6.19.: Moving-window averaged results from station OTVZ. The scale has been shifted for both shallow and deep events. 


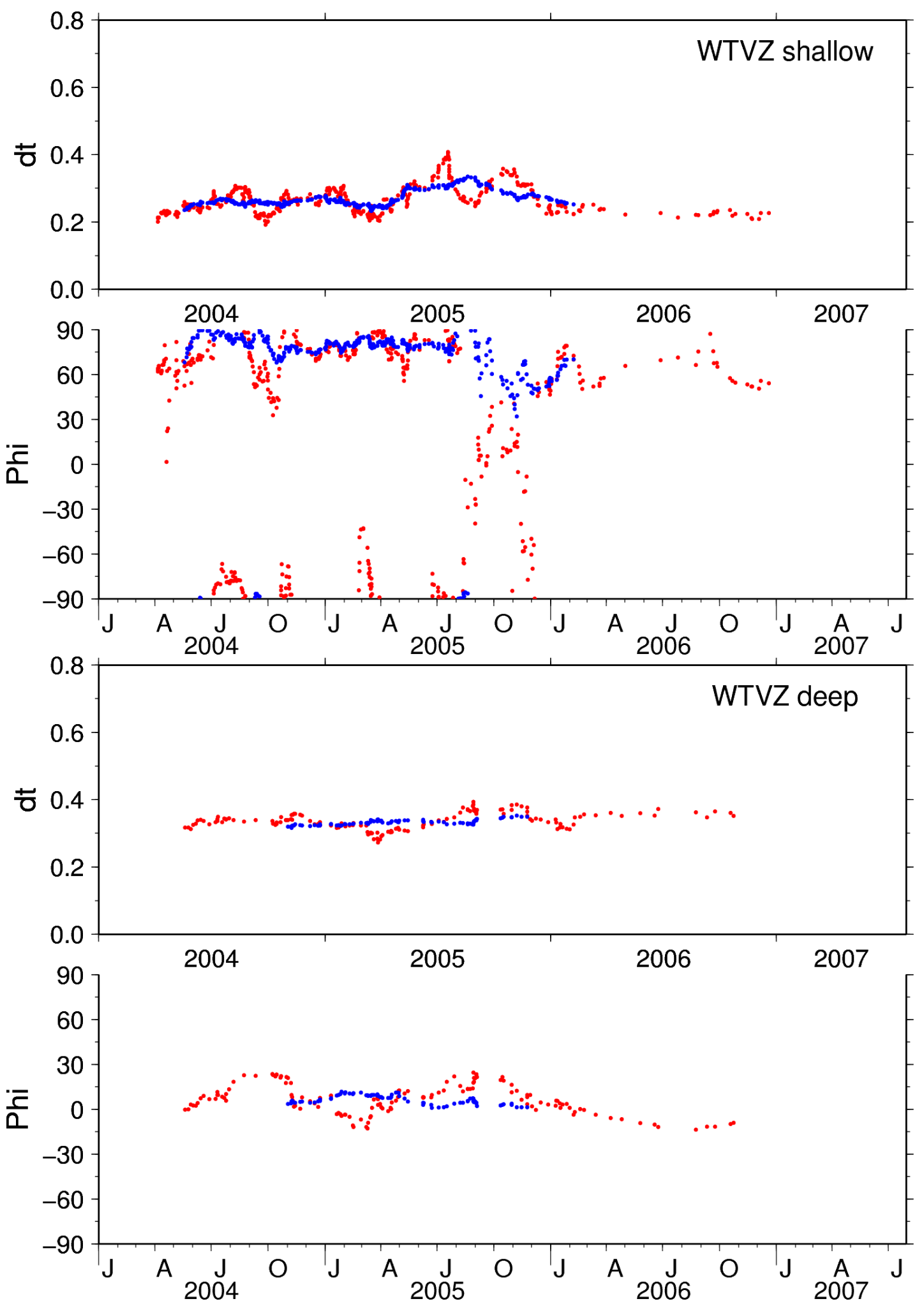

Figure 6.20.: Moving-window averaged results from station WTVZ. 


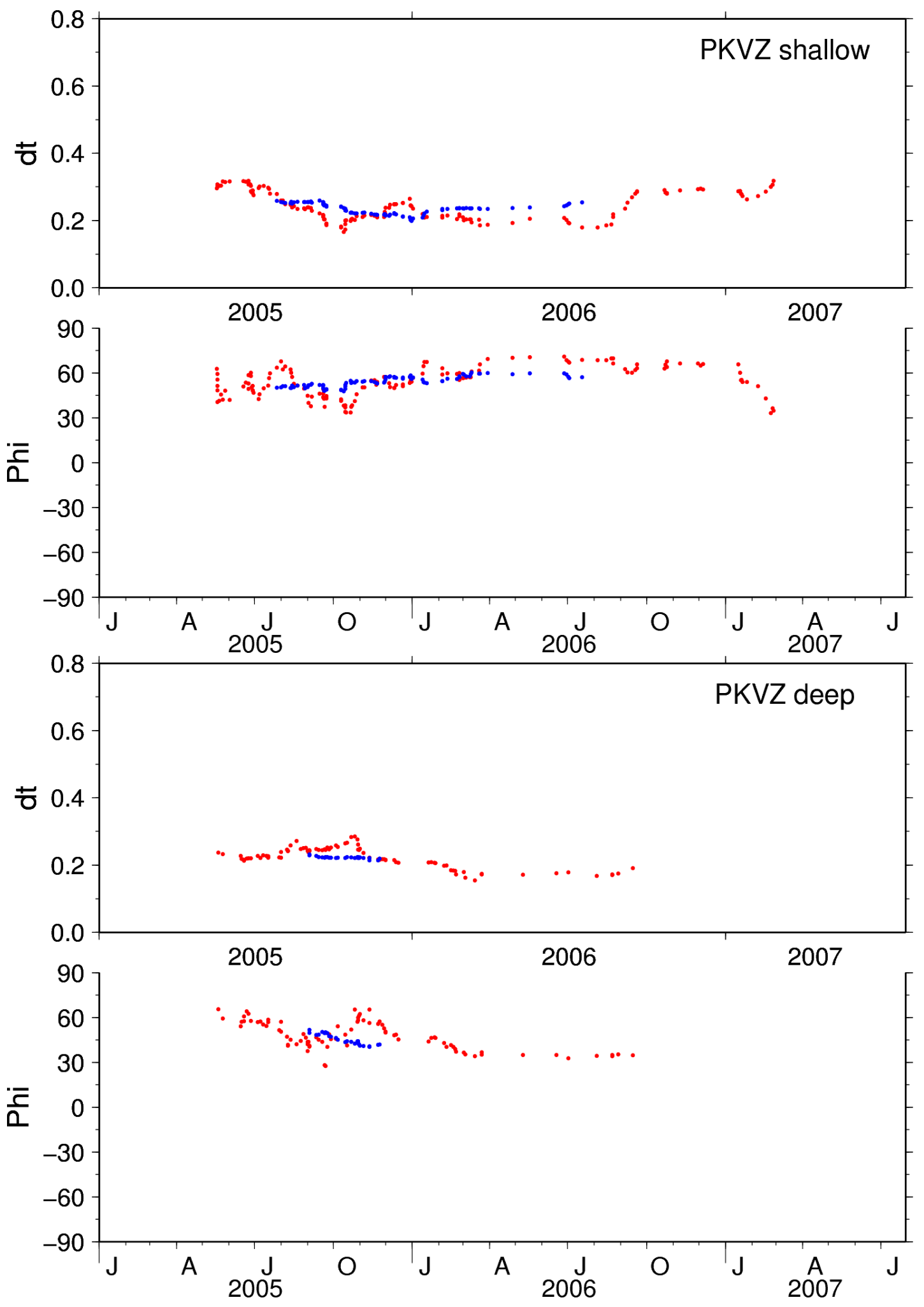

Figure 6.21.: Moving-window averaged results from station PKVZ. 


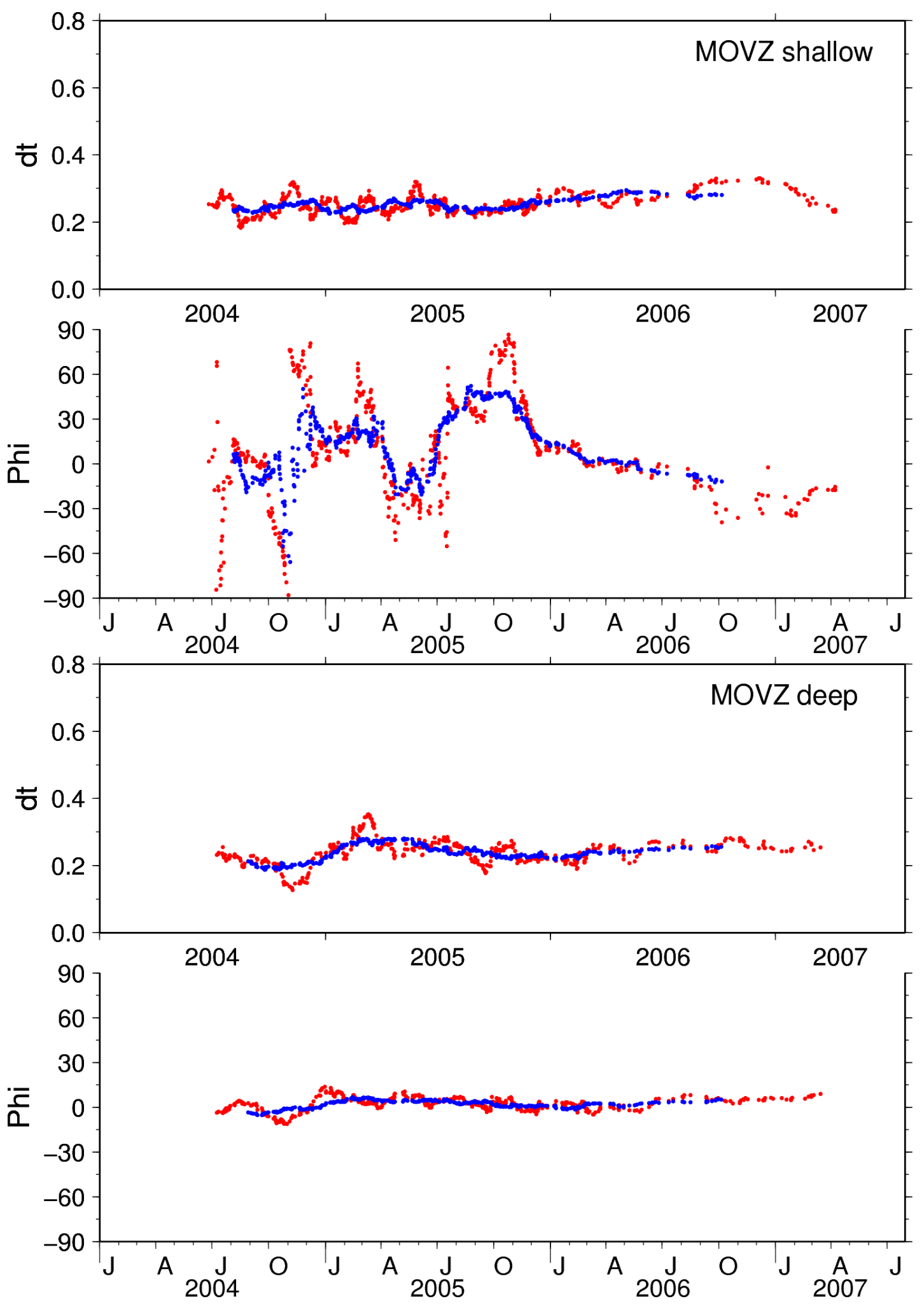

Figure 6.22.: Moving-window averaged results from station MOVZ. 


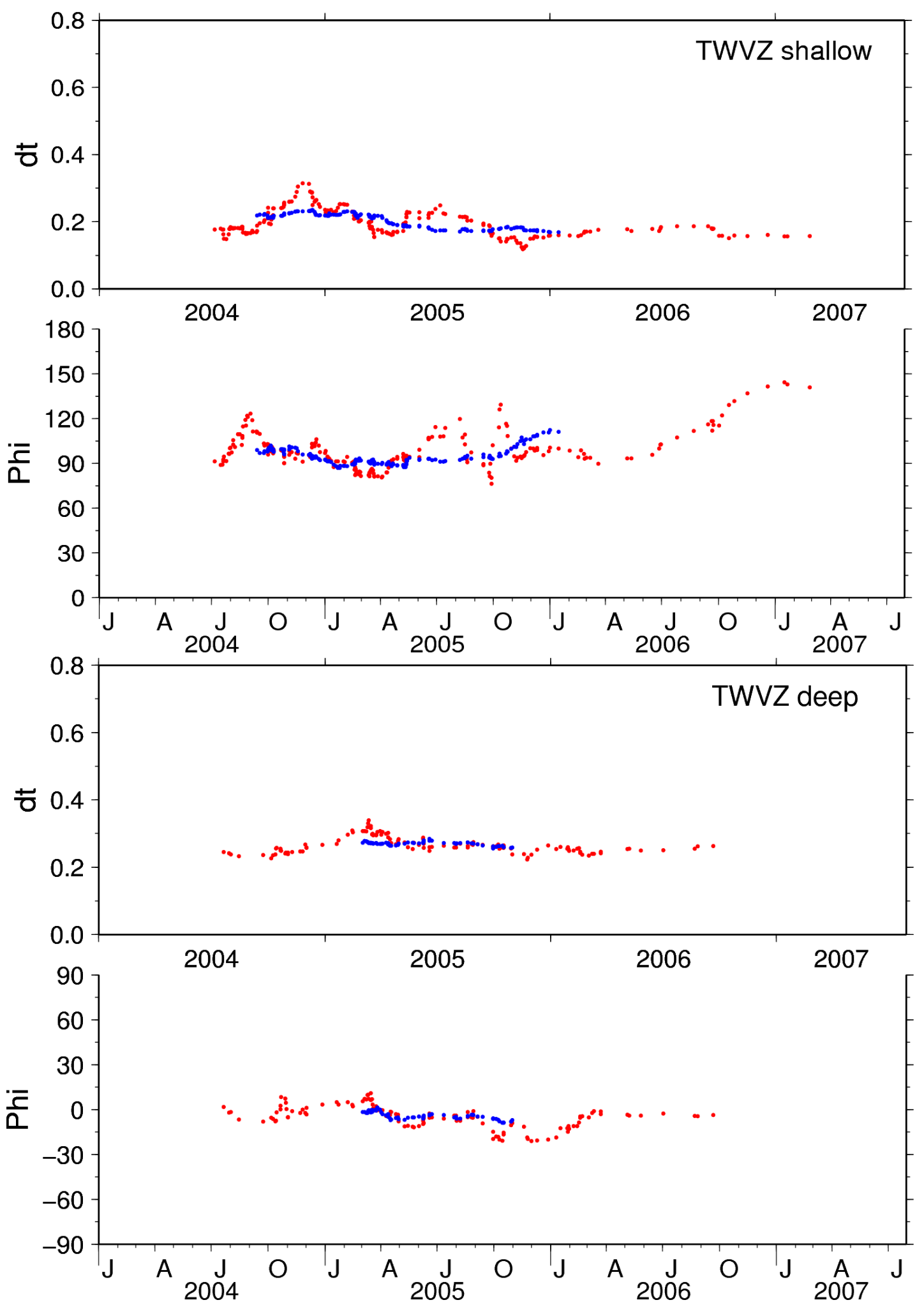

Figure 6.23.: Moving-window averaged results from station TWVZ. The scale has been shifted for shallow events. 


\section{Discussion}

\subsection{The newly developed automatic method}

The results from manual measurements could be fairly reproduced with the automatic technique ("aut"). By applying automatic quality control criteria rather than manually selecting good measurements, different data subsets compared to the ones from the manual measurements were often selected for the final result. Some events, which were rejected after manual inspection, met the criteria of the automatic quality control; some other events, which passed the manual inspection, were rejected by the automatic one. Still, the overall trends seen in the previous analyses of 1994, 1998 and 2002 data sets were mostly reproduced by the automatic technique (Figures 6.16.4 and Tables 6.1 and 6.2). For deep events the mean fast direction $\bar{\Phi}$ of reprocessed data from 2002 (all stations) was $19.7 \pm 5.5$, compared to $19.2 \pm 2.7$ determined by the manual measurements of (Gerst and Savage, 2004). For shallow events a mean fast direction of $-15.2 \pm 3.9$ is obtained, which differs about $15^{\circ}$ from the manual result $(-30.0 \pm 2.4)$.

A possible reason for this difference for the results from shallow events are the higher number of measurements from stations LHOR2 (Gerst: $\bar{\Phi}=-14.1 \pm 5.4^{\circ}$, 24 measurements; aut: $-13.8 \pm 8.1,32$ ) and LTUR2 (Gerst: $-17.9 \pm 3.7,11$; aut: $1.7 \pm 4.8,40$ ), which contributed more to the mean value for the automatic method. Station LHUT2 (Gerst: $\bar{\Phi}=-45.4 \pm 3.0^{\circ}, 31$ measurements; aut: $-49.6 \pm 5.0,27$ ) 
delivered $25 \%$ of all measurements for the manual method and therefore contributed heavily to the mean value, while the contribution to the automatic method was only $12 \%$ of all measurements.

Measurements obtained from selected high-quality data sets give more consistent results, because events that led to good manual measurements with another technique are more likely to also yield good measurements when processed automatically (e.g. Figure 6.1). This indicates that the processes of event selection, filtering, and the rejection of low-quality measurements are key steps in the automation of the processing. These findings are also in agreement with the results of Teanby et al. (2004a), who obtain very consistent results from a selected high-quality subset of data.

The application of bandpass filters was fully automated (Section 4.2.1). A manual inspection of the waveforms that were filtered with an automatically selected bandpass filter showed that the signal to noise ratio criterion is sufficient to find a good filter for most events (see Figure 4.3, p. 73 for an example). This was further tested by processing events that were manually filtered by Gerst (2003) and processing the same events with an automatically chosen bandpass filter. For GeoNet data (20032007) most automatically determined best bandpass filters were in the "medium" frequency range (for $58.8 \%$ of the data the selected filter has corner frequencies of 1-3, $1-5$ or $2-3 \mathrm{~Hz}$, compared to $34.7 \%$ with $1-8,2-6,3-8$ or $4-10 \mathrm{~Hz}$ and only $6.4 \%$ with lower corner frequencies of $0.1-1,0.2-2,0.2-3,0.3-3,0.5-4,0.6-3$ or $0.8-3 \mathrm{~Hz}$ ).

The rejection of low-quality or erroneous measurements is difficult to automate. Here, manual (visual inspection of waveform and particle motion) and automatic criteria (maximum error, maximum delay time, signal to noise ratio) are different. 


\subsubsection{Comparison with other methods}

The automatisation of shear wave splitting measurements has been a goal of several other studies (see Section 3.3). The new automatic technique presented and used in this thesis (hereafter referred to as aut), can be used to make fully automated shear wave splitting measurements on large data sets.

Evans et al. (2006) devised a method to automate splitting measurements for SKS phases, but $98 \%$ of the data are rejected. Peng and Ben-Zion (2004) reject $70 \%$ of their measurements with their automatic technique (see Section 3.3 for details). There is a trade-off between rejecting as many erroneous and null measurements as possible and not rejecting valid measurements because of too strict selection criteria.

Table 7.1 contains statistics from the processing of data from 2003-2006 with the newly developed aut-method. From 18,810 phases* that had an S-phase pick, 16,446 were processed to retrieve the shear wave splitting parameters in a measurement. (Data were not processed if no filter that led to a signal to noise ratio of at least 2 could be found.) $56.4 \%$ of these measurements were accepted, while $43.6 \%$ were rejected by quality criteria or because they were classified as null measurements. From all events of the 2003-2006 data that had an S-phase pick, 49.4\% resulted in a measurement that was accepted by the automatic technique.

aut automatically determines the best bandpass filter to get a sufficient signal to noise ratio, so that every event is filtered separately. This is faster than manually filtering the data (e.g. Gerst, 2003), but promises better results than the application of one fixed bandpass filter to all data (as done by Peng and Ben-Zion, 2004; Evans et al., 2006). The measurements are done with a modified version of the cluster analysis code of Teanby et al. (2004a). The original code allows one to either select

\footnotetext{
${ }^{*}$ The 8502 events shown in Figure 5.3 (p. 96) can be recorded on more than one station, so that the number of phases $(17,437)$ is higher than the number of events.
} 


\begin{tabular}{|c|c|c|c|c|c|c|}
\hline station & year & $\begin{array}{r}\text { S-picks } \\
\#\end{array}$ & $\begin{array}{l}\text { processed } \\
\%(\#)\end{array}$ & $\begin{array}{l}\text { nulls } \\
\%(\#)\end{array}$ & $\begin{array}{l}\text { rejected } \\
\%(\#)\end{array}$ & $\begin{array}{l}\text { good } \\
\%(\#)\end{array}$ \\
\hline \multirow[t]{2}{*}{ TRVZ } & 2005 & $\overline{3380}$ & $95.5(363)$ & $28.9(105)$ & (42.4 (154) & $57.6(209)$ \\
\hline & 2006 & 120 & $62.5(75)$ & $22.7(17)$ & $41.3(31)$ & $58.7(44)$ \\
\hline \multirow[t]{4}{*}{ FWVZ } & 2003 & 1373 & $73.3(1006)$ & $28.4(286)$ & $44.6(449)$ & $55.4(557)$ \\
\hline & 2004 & 1148 & $81.5(936)$ & $28.6(268)$ & $48.9(458)$ & $51.1(478)$ \\
\hline & 2005 & 1192 & $87.2(1039)$ & $26.9(280)$ & $45.6(474)$ & $54.4(565)$ \\
\hline & 2006 & 245 & $91.0(223)$ & $33.2(74)$ & $47.5(106)$ & $52.5(117)$ \\
\hline \multirow[t]{2}{*}{ WNVZ } & 2005 & 1269 & $81.7(1037)$ & $34.6(359)$ & $40.8(423)$ & $59.2(614)$ \\
\hline & 2006 & 233 & $87.1(203)$ & $24.1(49)$ & $47.3(96)$ & 52.7 (107) \\
\hline \multirow[t]{3}{*}{ TUVZ } & 2004 & 592 & $79.7(472)$ & $22.9(108)$ & $47.7(225)$ & $52.3(247)$ \\
\hline & 2005 & 1106 & $85.0(940)$ & $26.3(247)$ & $48.2(453)$ & $51.8(487)$ \\
\hline & 2006 & 193 & 87.6 (169) & $27.2(46)$ & $50.3(85)$ & $49.7(84)$ \\
\hline \multirow[t]{3}{*}{ WPVZ } & 2004 & 891 & $82.9(739)$ & $19.2(142)$ & $40.9(302)$ & $59.1(437)$ \\
\hline & 2005 & 855 & $89.0(761)$ & $20.9(159)$ & $38.2(291)$ & $61.8(470)$ \\
\hline & 2006 & 208 & $87.5(182)$ & $24.7(45)$ & $41.8(76)$ & $58.2(106)$ \\
\hline \multirow[t]{3}{*}{ NGZ } & 2004 & 531 & $86.3(458)$ & $24.0(110)$ & $46.3(212)$ & $53.7(246)$ \\
\hline & 2005 & 553 & 89.9 (497) & 40.0 (199) & $43.9(218)$ & $56.1(279)$ \\
\hline & 2006 & 101 & $93.1(94)$ & $30.9(29)$ & $51.1(48)$ & $48.9(46)$ \\
\hline \multirow[t]{2}{*}{ MTVZ } & 2005 & 759 & $91.2(692)$ & $26.2(181)$ & $45.4(314)$ & $54.6(378)$ \\
\hline & 2006 & 244 & $90.2(220)$ & $30.0(66)$ & $44.5(98)$ & $55.5(122)$ \\
\hline \multirow[t]{3}{*}{ OTVZ } & 2004 & 59 & $89.8(53)$ & $35.8(19)$ & $49.1(26)$ & $50.9(27)$ \\
\hline & 2005 & 698 & 90.7 (633) & $27.0(171)$ & $49.4(313)$ & $50.6(320)$ \\
\hline & 2006 & 154 & 90.3 (139) & $23.7(33)$ & $36.7(51)$ & $63.3(88)$ \\
\hline \multirow[t]{3}{*}{ WTVZ } & 2004 & 584 & $83.2(486)$ & $19.3(94)$ & $36.8(179)$ & $63.2(307)$ \\
\hline & 2005 & 623 & $87.2(543)$ & $16.2(88)$ & 33.3 (181) & 66.7 (362) \\
\hline & 2006 & 139 & $85.6(119)$ & $16.8(20)$ & $31.9(38)$ & $68.1(81)$ \\
\hline \multirow[t]{2}{*}{ PKVZ } & 2005 & 451 & $93.8(423)$ & $29.3(124)$ & 46.8 (198) & $53.2(225)$ \\
\hline & 2006 & 173 & $94.8(164)$ & $28.0(46)$ & $46.3(76)$ & $53.7(88)$ \\
\hline \multirow[t]{3}{*}{ MOVZ } & 2004 & 947 & $96.2(911)$ & $29.7(271)$ & $54.8(499)$ & $45.2(412)$ \\
\hline & 2005 & 1623 & $96.9(1573)$ & $31.0(487)$ & $52.4(824)$ & $47.6(749)$ \\
\hline & 2006 & 457 & $98.2(449)$ & $30.7(138)$ & $48.3(217)$ & $51.7(232)$ \\
\hline \multirow[t]{3}{*}{ TWVZ } & 2004 & 338 & $93.2(315)$ & $29.2(92)$ & $47.3(149)$ & $52.7(166)$ \\
\hline & 2005 & 443 & $92.6(410)$ & $26.3(108)$ & $46.3(190)$ & $53.7(220)$ \\
\hline & 2006 & 128 & $95.3(122)$ & $33.6(41)$ & $45.1(55)$ & $54.9(67)$ \\
\hline ALL & 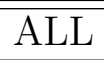 & 18810 & $87.4(16446)$ & $28.5(4691)$ & $\overline{c 43.6(7163)}$ & $\bar{~} 56.4(9283)$ \\
\hline
\end{tabular}

Table 7.1.: Statistics of the automatic processing of GeoNet data (2003-2006). For each station (sorted by distance from Ruapehu) and year this table lists: the number of phases that had an S-Pick, the number of events for which a filter with a signal to noise ratio of at least 2 could be found and that were used for a splitting measurement (processed), the number of measurements that were classified as nulls, the number of measurements that were rejected (low quality measurements and nulls), and the number of measurements that met all quality criteria ("good"). Numbers are given in per cent and absolute values (\#, in parentheses). The percentage of processed events is based on the number of events with an S-pick. All other percentages are based on the number of measurements ("processed"). 
minimum and maximum window times interactively or to use one set of window times for all measurements. I automated the selection of cluster analysis parameters (including the window times) on a per-event-basis, again to allow faster (automatic) processing compared to manual selection and also to retrieve better results than with a "one-off" approach. See Figure 4.1 for an overview of the processing steps and Chapter 4 for details)

\subsubsection{Caveats of this new method}

The newly developed technique of automatic measurements of shear wave splitting can not replace careful manual investigation of the results by the analyst. Automating the complete measurement process introduces various error sources and results tend to be more scattered and less consistent than results obtained by manual measurements. However, even with these additional error sources, the results are constant over several years for most stations (Figures 6.6-6.10). Results were found to be consistent even for sparse data sets, when only high-quality events that gave good results in previous studies were processed. Without this preselection step, erroneous events introduce inconsistencies to the results and enforce the use of larger data sets.

The only requirement to use the automatic method for shear wave splitting measurements is an S-phase pick for each event. Small offsets of the S-pick are not critical, because different measurement windows (relative to the S-pick) are tested and the most stable configuration is selected with a cluster analysis. S-picks could be calculated from P-picks if the $v_{p} / v_{s}$-ratio can be estimated. However, inexact calculated S-picks introduce another source of error to the automatic processing and manual S-picks are preferred. Only results from events with manual S-picks are used in this study. 


\subsection{Changes between 2002 and the new data set (2003-2007)}

The comparison of results from the 2002 data set and from new GeoNet data is not straightforward. Changes between these data sets can be influenced by several factors:

1. change of method

2. change of sources

3. change of stations

4. changes of the anisotropic properties in the investigated area

Since the previously analysed data sets have been reprocessed, results that were obtained with the same method can be compared. This leaves points $2-4$ to explain changes in the results between 2002 and more recent data. Results have been found to show high variations between different stations (Figures 6.4-6.10), but a comparison of adjacent CHARM- and GeoNet stations shows similar trends (e.g. LTUR2 and TURO2 compared to TRVZ; Figures 6.4 and 6.7). Station FWVZ gave results (although highly scattered) that are consistent within the standard error for data from 2002 and 2003.)

\subsection{Temporal changes within the new data set}

No major significant temporal changes in anisotropy in the available data sets from 2003-2007 have been detected with the method used in this study (see Figures 6.66.9). Although results vary from station to station, most stations gave consistent results over a few years of data. Stations MOVZ, TUVZ and NGZ obtain consistent fast orientations from deep events over several years of data and show a NE-SW trend of fast orientations, which coincides with the regional maximum horizontal 
stress. Results from shallow events seem top be more scattered for most stations. Combined results of 3 years (2004-2006) are shown in Figure 6.10 (p. 113).

The scatter of single measurements can effectively hide any temporal trends in the data. By calculating moving-window average values, long-term trends can be distinguished from short-term scattering (Figures 6.12-6.23, p. 115).

Results from 2007 data are often found to be aberrant from results of earlier years (see Table 6.3). This can be explained by the sparseness of most 2007 data sets.* For example the mean fast orientation measured from deep events at station TRVZ differs between 2006 and 2007 by $32.2^{\circ}$ (Table 6.3). But only 5 measurements in 2007 passed all processing steps and contributed to the mean value, resulting in a high standard error and a mean value that could be entirely changed by adding few new measurements to the data set.

If only data sets with at least 30 events are considered, the mean value of the fast orientation changed for three stations between 2004 and 2006:

- MTVZ, shallow events: $88.1 \pm 10.6$ (in 2005) to $45.2 \pm 15.3$ (in 2006); a change of $42.9^{\circ}$

- MOVZ, shallow events: $5.7 \pm 15.2$ (2004) to $23.8 \pm 6.9$ (2005) to $0.2 \pm 6.4$ (2006); changes of 18.1 (although within the standard error) and $23.6^{\circ}$

- PKVZ, deep events: $57.4 \pm 9.3(2005)$ to $33.9 \pm 4.9$ (2006); a change of $23.5^{\circ}$

The change at station PKVZ could be related to a change of sources, because the backazimuthal distribution of events was different for this station for the two years in which the change in the mean fast orientation occurred (see Figures C.19 and C.20, p. 173). But no such change of the sources could be observed at the other two stations (see Section 7.4). Changes of the mean fast orientation with time are visible on some of the moving-average plots. Again there are no significant major changes in

*Events have only been considered up to July 2007. An additional effect is the delay in the picking of S-phases for the GeoNet data, where not all event phases are picked instantly. 
the data that can definitively be attributed to actual changes in anisotropy. Results from some stations are scattered, while other stations show stable results over time.

At station FWVZ the fast orientations seem to change with time (with episodes of change at the same time for shallow and deep events; see Figure 6.13), but the confidence in non-randomness is not very high for this station (Table 6.3, low $|\vec{R}|$ values).* Similarly, at station WPVZ results are very scattered (Figure 6.16), but a detailed investigation of the apparent changes of fast orientations in 2005 would be interesting for future analysis. Results from deep events at station NGZ do not change with time, but results from shallow events are highly scattered (Figure 6.17). Results at station MTVZ are different at the end of 2005 than for 2006 and 2007 (Figure 6.18). For shallow events there is a trend of NW-aligned fast orientations west of Ruapehu and NNE-aligned orientations and the rose diagrams of this station indicate a bimodal distribution of fast orientations (Figures 6.8 and C.12).

The apparent temporal changes at station MOVZ (shallow events, Figure 6.22, p. 125) can be explained by spatial variations of the splitting parameters (see Figure 7.1). Data are aligned approximately ENE for events that came from east or west of Ruapehu, while they are NNE-aligned south of Ruapehu. This bimodal spatial distribution of fast orientations is also visible in the rose diagram of this station (see Figure 6.10 p. 113).

As has been discussed in Section 3.3, delay times in shear wave splitting studies of crustal events are generally expected to be scattered. However, delay times obtained with the automatic technique were in good agreement with manually measured delay times (see Figure 6.1). The mean values of the delay times measured with the new data sets did not vary significantly between 2004 and 2006, but varied from station to station (Table 6.3). For data sets of at least 30 events, an increase in the mean delay time of shallow events occurred at station OTVZ from 2005 to 2006 (0.23 to

\footnotetext{
*Furthermore, (Gerst, 2003) reported high scatter and anomalous effects at FWVZ.
} 


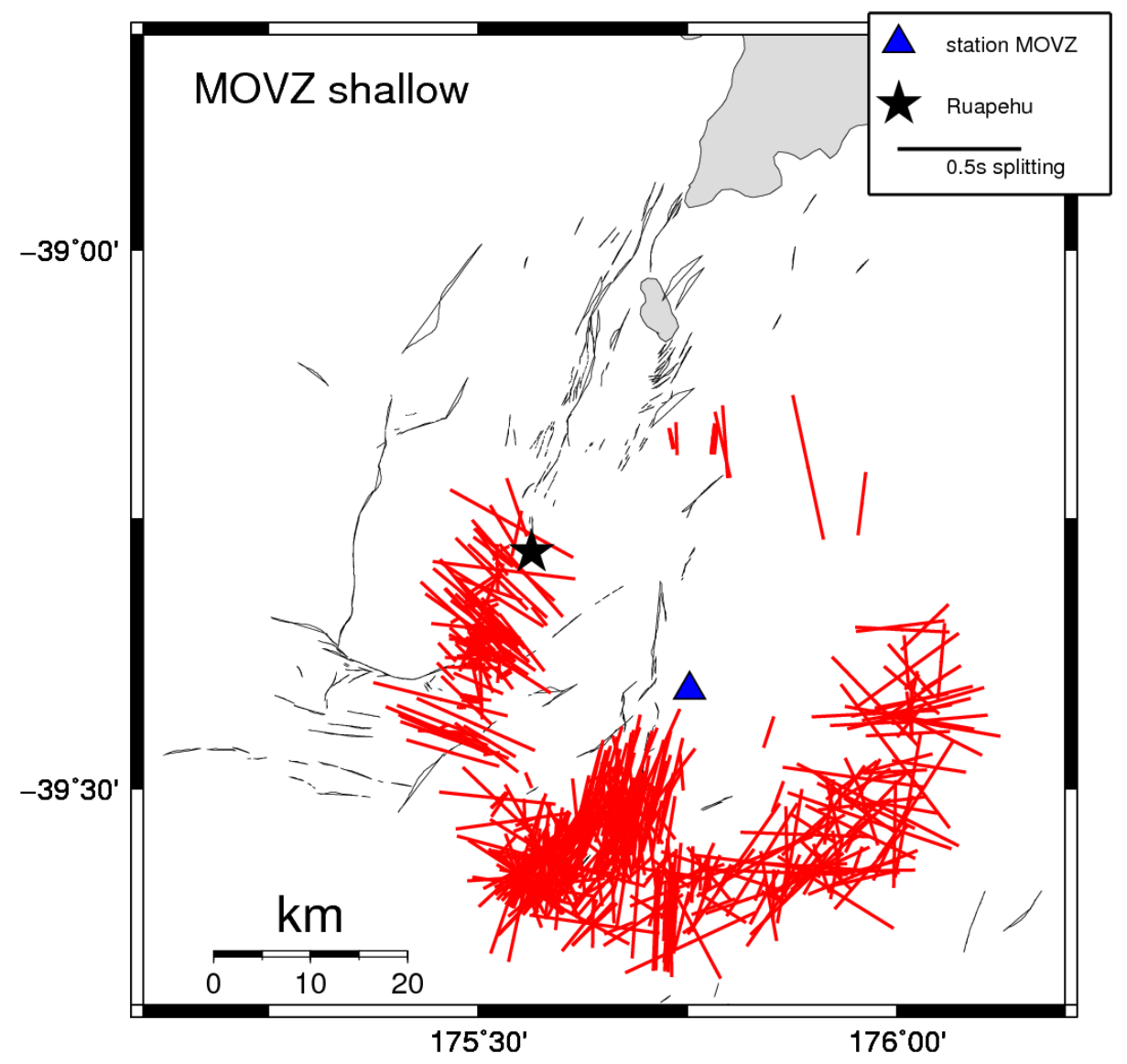

Figure 7.1.: Results from station MOVZ (shallow events, data from 2004-2006). See text for details.

$0.37 \mathrm{~s})$, but standard deviations of the delay times are generally high $(0.16$ and $0.20 \mathrm{~s}$ for OTVZ 2005 and 2006).

Moving-window averages of the delay times indicate that delay times are more scattered for shallow events than for deep events. Delay times for deep events are mainly constant (Figures 6.13-6.23) and Table 6.3). An increase in delay times of about $0.1 \mathrm{~s}$ can be seen at stations MOVZ and WNVZ between 2004 and 2005 for deep events (both stations are located SE of Ruapehu, but station MOVZ is more than $20 \mathrm{~km}$ away from Crater Lake). Delay times from shallow events vary with time at stations FWVZ, TUVZ, TWVZ, WNVZ and WTVZ, but no systematic increase or decrease is seen (the variations have a wavelength of 1-3 months). Figures 6.17 and 6.19 show a gradual increase of delay times for shallow events at stations NGZ and 


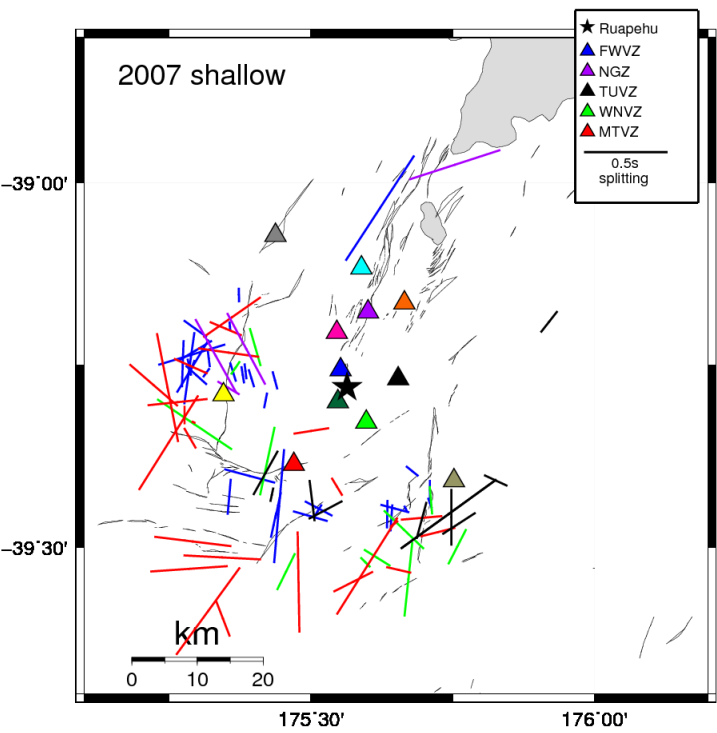

(a) shallow

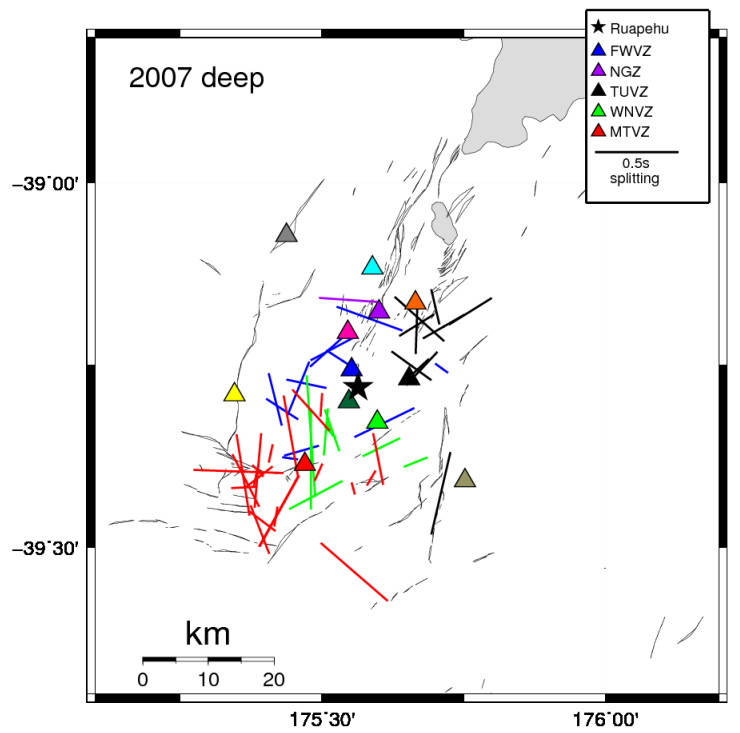

(b) deep

Figure 7.2.: Results from 2007 data for stations FWVZ, NGZ, MTVZ, TUVZ and WNVZ. The bars represent splitting parameters and are plotted at a projected depth of $50 \mathrm{~km}$. Faults are shown as thin grey lines.

OTVZ (which are both located about $5 \mathrm{~km}$ apart at Mt. Ngauruhoe, NE of Ruapehu). Variations in delay times of shallow events at station WPVZ in 2004 and 2005 (Figure 6.16) coincide with changes in the fast orientation at the same time, but the results from this station are very scattered (Figures 6.6 and 6.7).

\subsection{Spatial changes within the new data set}

To investigate spatial trends in the results the single measurements are plotted on a map as a bar, where the length of the bar represents the delay time and the orientation of the bar represents the orientation of the fast shear wave. Single measurements from 2007 data from the stations FWVZ, NGZ, MTVZ, TUVZ and WNVZ are shown in Figure 7.2. For deep events, measurements from stations FWVZ, WNVZ and MTVZ are in agreement in the area west-southwest of Ruapehu (aligned NNW). For shallow events, fast orientations from stations NGZ and WNVZ are aligned NNW in the area west of Ruapehu, while several groups of measurements from different stations show 
similar trends in the area south of Ruapehu. For both deep an shallow events the measurements often seem to be aligned with fault lines.

For a continuation of this study, the analysis of spatial trends in the results could help to improve the quality of results, because events from areas that give highly scattered orientations could be treated separately. Split-maps for all years and all stations are shown in Appendix C, 163.

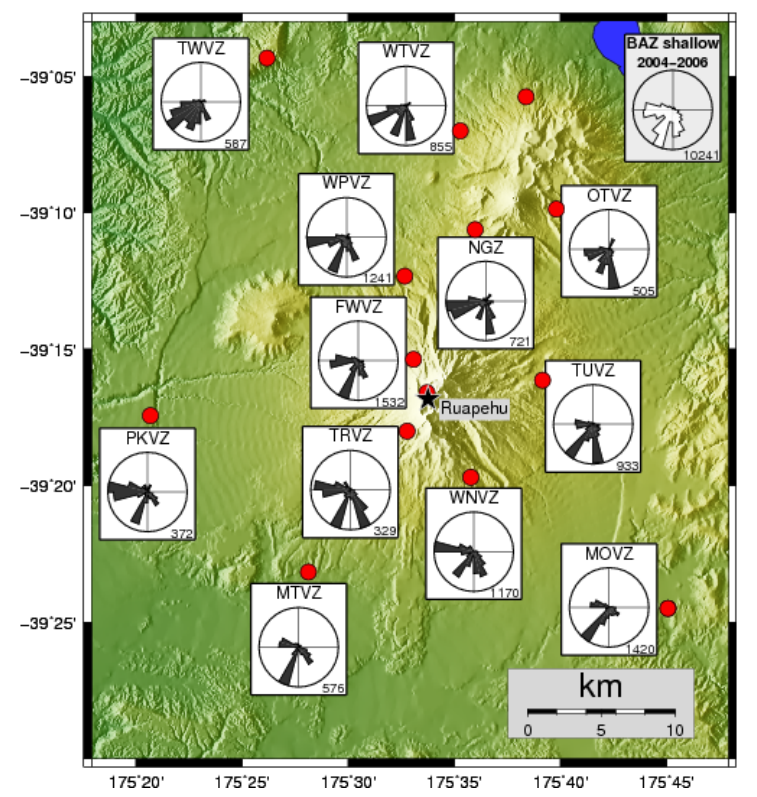

(a) shallow events $(0-30 \mathrm{~km})$

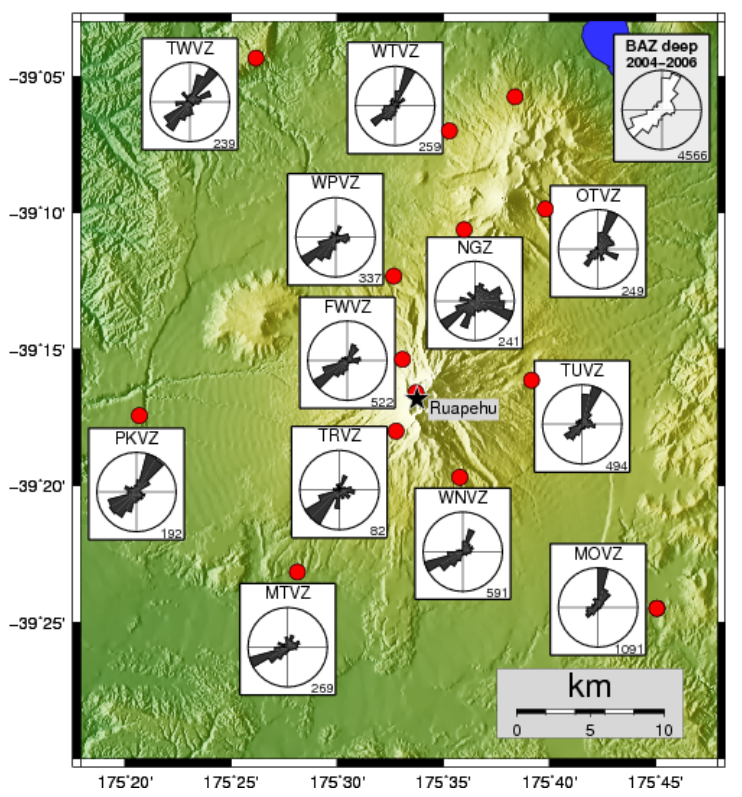

(b) deep events $(55-300 \mathrm{~km})$

Figure 7.3.: Backazimuth of selected event data per station in 2004-2006. Unlike Figure 5.3, only data with a signal to noise ratio of three or greater (after the application of a filter) are considered for the histograms.

The backazimuthal distribution of events is shown in Figure 7.3. This figure indicates the spatial distribution of events that led to measurements with acceptable quality (namely the measurements that were shown in Figure 6.10). Although many shallow events originate in the TVZ, NE of Mt. Ruapehu (Figure 5.3, p. 96), the histograms of backazimuthal distribution indicate that most "usable" events originate east to south of Ruapehu. Many events that originate in the TVZ travel through damaged volcanic rocks and are more likely noise-contaminated, or S-waves are attenuated when travelling through this highly attenuative region (e.g. Eberhart-Phillips et al., 
2005; Reyners et al., 2006). These events are then rejected by the automatic quality control because of low signal to noise ratio or because of the absence of any clear S-wave arrival and this leads to the comparatively small number of NE-originating events (Figure 7.3).

The backazimuths differed between deep and shallow events, but only minor variations at a single station are seen with time, so that Figure 7.3 is representative for the distribution of sources for all data sets. Yearly maps of the backazimuthal distribution are shown in Appendix C.3 (Figures C.18-C.21, p. 172). 


\section{Conclusions}

\subsection{Summary of the results}

A method has been developed to fully automate shear wave splitting measurements. Starting with a list of earthquakes, the software package automatically retrieves data from the GeoNet FTP server, produces SAC files and prepares these files for splitting measurements. The best bandpass filter is chosen for each event based on a signal to noise ratio criterion. The measurement itself is carried out with the program ass (Teanby et al., 2004a) that is based on a grid-search algorithm (Silver and Chan, 1991). Measurement window times are calculated from the S-pick time and dominant period of the wave. The results of each measurement are tested for several quality criteria and are illustrated by maps of rose diagrams and individual measurements.

Starting with triplets of SAC files the data can be processed with a single command line and without any manual interaction. About 100 phases can be processed in one hour.* Approximately $50 \%$ of all S-phases are rejected as null measurements or low quality events, which leaves $50 \%$ of accepted results.

The automatic technique has been applied to GeoNet data recorded in 2003-2007 to investigate possible temporal changes in anisotropy. Almost 10,000 events, often recorded on multiple stations, have been processed and led to several thousand mea-

*Estimated processing time for the GeoNet data used in this study on a machine with a $1.8 \mathrm{Ghz}$ CPU and 1 GB RAM. 
surements. No significant major temporal changes in anisotropy between 2004 and 2007 have been detected.

\subsection{Prospects}

\subsubsection{This method as a monitoring tool at Mt. Ruapehu}

Since numerous GeoNet stations are deployed continuously in the vicinity of Mt. Ruapehu and their data are easily accessible, automatic shear wave splitting measurements at Mt. Ruapehu can be carried out in the future without much effort. However, the automation of the measurements introduces an additional source of uncertainties and cannot fully replace careful manual analysis. On the other hand, the method can be easily used to process large data sets with fast and reproducible results.

The automatic method can be used as a preliminary monitoring tool of seismic anisotropy at Mt. Ruapehu. It helps to determine subsets of data which seem worthwhile to be investigated in greater detail. Most stations showed no or only little variation of the shear wave splitting parameters over time (Chapter 6) and continuous monitoring of future data from these stations can help to detect possible changes in anisotropy in the area.

Based on the results obtained in this study I suggest for further analysis of anisotropy in the vicinity of Mt. Ruapehu:

- Continuous monitoring of shear wave splitting with the automatic method: This can be done without much effort and is especially interesting for stations that gave consistent results over three years of data and where no temporal changes have yet been observed. 
- Stations that only obtained very few measurements should be considered for manual or semi-automatic analysis (e.g. Gao et al., 2006). Stations TRVZ and NGZ each gave only 16 accepted measurements from deep events since 2006. Manual analysis of these sparse data sets could increase the number of measurements to get reliable results for these stations. The few results obtained from TRVZ (which is the closest station to Crater Lake except station DRZ) in 2006 indicate an alignment of fast orientations with the regional maximum horizontal stress. An apparent change of the fast orientations at station TWVZ (Figure 6.23) is based on only very few data and should be confirmed by further analysis of additional events.

- Sparse data sets could also be enlarged by manual picking of S-phases and manual filtering.

- Split maps of several stations (FWVZ, WNVZ, TUVZ, WPVZ, MTVZ, and MOVZ; Figures C.7, C.8, C.9, C.10, C.12 and C.16) indicate highly scattered results for shallow events from a region SSW of Ruapehu. To separately analyse all events from a backazimuthal range of about $190^{\circ} \leq \mathrm{BAZ} \leq 230^{\circ}$ could help to reduce the scatter of results commonly observed on many stations.

- For stations that gave varying results with time, a detailed analysis is necessary to confirm or possibly reject the automatically determined results. A manual quality control step would help to distinguish real bimodal results from null measurements that cause results to appear bimodally distributed. Based on the results of this study, a detailed analysis of data from MTVZ (bimodal fast orientations), OTVZ (apparent increase of delay times) and deep events from station WPVZ (changes of fast orientations in 2005) should be carried out.

- Repeating events (multiplets) could be used as well-characterised and stable sources to investigate any temporal changes in greater detail. 


\subsubsection{This method at other localities}

Although some steps (e.g. data retrieval) have been specifically designed for data from the GeoNet network, the method can be used to automatically measure shear wave splitting with other data sets. Shear wave splitting measurements with local earthquakes are used in many studies (e.g. Crampin and Peacock, 2005, and references therein). The only general requirement to gain data for shear wave splitting studies is the use of three-component seismometers. Apart from an S-phase pick that needs to be selected or calculated for each event, there are no further requirements to use another data set for processing with the automatic shear wave splitting method.

\subsubsection{What can be improved?}

The method presented in this thesis is a technical solution for automatic splitting measurements. When applied to carefully selected subsets of data that gave good results when investigated with manual techniques, the results from the automatic method are in very good agreement. If applied to a complete data set that includes noisy events and null measurements, the results are more scattered and interpretation is difficult.

The quality of results from this study can be improved by a manual quality control of measurements (e.g. by visual inspection of an analysis plot). This would help to reject null measurements and low quality measurements that were not detected by the automatic quality control criteria. 


\section{A. User Manual: Software used and developed for this study}

To process large amounts of seismograms it was essential for this study to automate the work-flow as much as possible. Several shell scripts, GMT (Wessel and Smith, 1991) scripts and SAC (Goldstein and Snoke, 2005) macro files have been developed to automate processes with the use of computer power, especially when the tasks were recurrent. When writing these scripts, the UNIX philosophy was followed, so several small tools were used in combination to deal with complicated tasks. For shear wave splitting measurements, events have to be selected and processed, and measurements have to be evaluated and displayed. A description and manual of the developed programs is given here. Many programs can most conveniently be invoked by the main program doass and its options, but it is also possible to do the processing step by step. To get a quick overview of what to do, see the cheatsheet (p. 158). Shell commands, i.e. commands that have to be entered in a terminal are indicated by formatted text and/or a prepend $\$$-sign, e.g. $\$$ whoami means that you are supposed to type whoami in a terminal to execute this command.* Event names and filenames are also emphasised.

\section{A.1. Basic knowledge of geophysical terms}

The programs described in this manual are used to measure shear wave splitting to retrieve information about the anisotropy of a medium. Besides the main text of this thesis, the shear wave splitting tutorial of Savage (1999) and the original publications on the shear wave splitting measurement algorithm (Silver and Chan, 1991) and the cluster analysis method (Teanby et al., 2004b) are recommended for further reading.

\section{A.2. Prerequisites to use the programs}

The scripts to use the method can be found on the attached CD-ROM or in the network at Victoria University at tararuas.geo.vuw.ac.nz:/Users/home/awbochum/ doass. Besides basic Unix tools the code requires some software packages to run:

SAC: Seismic analysis code. Version 100.X or greater is highly recommended. Obtain from http://www.iris.edu/manuals/ (visited Aug 8, 2007).

${ }^{*}$ The command whoami prints the username to the screen and is only used here as an example. Note that \$string without a space between the dollar sign and the string denotes a shell variable. 
TauP: Seismic traveltime utilities. Version 1.1.7 is recommended.

Obtain from http://www.seis.sc.edu/software/TauP/ (visited Aug 8, 2007).

rdseed: SEED file reader. Version 4.5 or greater is recommended. Not needed if the data are already in SAC format.

Obtain from http://www.iris.edu/manuals/ (visited Aug 8, 2007).

GMT: Generic Mapping Tools. Version 4.2.X or greater is highly recommended. Obtain from http://gmt.soest.hawaii.edu/ (visited Aug 8, 2007).

The doass/bin directory needs to be included in the $\$ P A T H$ variable of the shell's environment so that the programs can be found by the shell. To find out quickly if all required programs are installed properly, run doass_precheck. This script prints a warning message to the screen if a program is missing or obsolete:

\$ doass_precheck

\section{A.2.1. Customise paths and variables}

The most commonly used settings and paths can easily be set by the user in the file doass_config. Some parameters, such as the number of measurement windows for the cluster analysis, have a default value and do not necessarily have to be adjusted. Paths, however, need to be set up on each machine to run the programs, so that macros (subfolder doass/macros) and shell scripts and binaries (subfolder doass/bin) can be found by the programs. The script doass_precheck will print an error message if the paths to the required directories and files are not set properly.

\section{A.2.2. Programming style and syntax}

A few basic shell scripting techniques (using BASH syntax) should be known for better understanding and easier use of the programs:

awk syntax: awk is a pattern scanning language that is used to extract certain content from logfiles. To read the second and third column of a comma-delimited text file (data.txt), the command <data.txt awk $-\mathrm{F}$, ' \{print $\$ 2, \$ 3$ \}' is used.

for-loops and processing of many events: Most of the programs and scripts are designed to process one event at a time. When processing many events at a time, a for-loop is used to call the program for each event until all events have been processed. If the command doass_findfilt -f eq1 is used to run the program findfilt on the event eq1, then for $j$ in *.e; do doass_findfilt $-f \$\{j \% . e\}$; done will invoke the program for all events in the current directory. This might come with a loss of processing speed when handling many events at once (e.g. because programs like SAC have to be started and quit each time the for-loop is passed through), 
but allows straightforward separate processing of single events or custom subsets of events. The $\$\{j \%$.e $\}$ construct is used to strip the suffix .e from the filename.

command substitution: Variables are often set to the output of a command with the use of backticks. When using wordcount='cat data.txt | wc -w', the variable wordcount will be set to the output of the given command within the backticks (in this example, cat opens the file and wc counts the words in the document). Note the difference between regular ticks (') and the backticks (') used in this example.

\section{A.3. Download and convert data}

This section explains the process of downloading and converting data from the GeoNet network. If any other data are investigated, this section can be skipped (go to Appendix A.4 below). Starting with a comma separated values list (filename with suffix .csv) that can be retrieved from the GeoNet Earthquake search website*, all data files are downloaded in SEED format from the GeoNet FTP server for a given list. It is possible to give a maximum distance (from Mt. Ruapehu ${ }^{\dagger}$ ) and a magnitude range to exclude events that do not meet these criteria. The script also extracts (-e option) some event details to a file (the file has a . info suffix and contains CUSP ID, year, day of year, latitude, longitude, magnitude and depth (in km), e.g. 2668211, 2006, 349, -37.2154, 179.96463, 3.818, 33). Empty files with an extension .wanted are created for each file that is to be downloaded. These placeholder files are deleted if the download of an file is complete.

\$ gns_getevents.sh

Run without any options to display a help message.

\$ gns_getevents.sh -f block2.2006.deep.csv -e -s 2 -d

To download (-d option) all events that are listed in block2.2006.deep.csv (-f option to pass a filename to the program) and meet the criteria of block2 (-s option to select a block; see Table 4.1). The option -e is used to extract a short list whose content is then passed to the tool wget to load the data.

\$ gns_getevents.sh -f block2.2006.deep.csv -s 2 -d

To resume downloading data from the same list as in the example above or if you want to download (-d) separately from making the list (-e). If a large amount of data has to be transferred, the downloading can be interrupted and resumed later.

*http://magma.geonet.org.nz/resources/quakesearch/, visited Jul 27, 2007

$\dagger$ This can be changed if the programs are used to investigate other areas. Distances are calculated with the script distance_ruapehu and the area of interest can be changed in this script by providing another latitude-longitude pair. 


\section{Extract SAC files}

A SEED file contains all recorded data from many GeoNet stations for a single event. Especially events with a high magnitude are usually recorded on stations throughout New Zealand, out of which only a handful are located in the vicinity of Mt. Ruapehu. With the shell script seed2sac, data files for each component $(e, n, z)$ of each single station are extracted, renamed, and stored in SAC format. The tool rdseed* is used to extract SAC files from a SEED file. An additional file (.info) with event information such as magnitude and depth is created for every single event. After extraction of the data the SEED file is zipped to save disk space. Data recorded at distant stations, data with no S-phase pick and data which is not three-component data are moved to an archive or can be deleted. It should be made sure that there is sufficient free disk space.

$\$$ seed2sac

Run without any options to display a help message.

$\$$ seed2sac -f 2508375

Extract SEED file with CUSP ID 2508375. ${ }^{\dagger}$ The filename of the SEED file has to be in the format xCUSPID.seed, e.g. x2508375. seed.

\$ seed2sac -s quake3. seed (not recommended, see text)

Extract the SEED file quake3.seed. The CUSP ID will be set to 1111111. This option should only be used for non-Geonet data that is not in the xCUSPID.seed format. Instead of using this option it is recommended to rename SEED files and assign a manual 7-digit number as pseudo CUSP ID (x0000001. seed, x0000002. seed etc.), which makes maintenance of the data easier.

\$ seed2sac -f 2508375 -1/seismo/statlist

To distinguish stations in the region of interest (here: in the vicinity of Mt. Ruapehu) from distant stations, a station list has to be provided. This list should consist of rows with four columns each: station name, station latitude, station longitude, distance from the region of interest in $\mathrm{km}$ :

FWVZ -39.25670 175.55276 2.87006

MTVZ -39.38636 175.4702314 .223

TUVZ -39.26943 175.653987 .85138 etc.

It is probably more convenient to enter the path to this list (and the maximum distance of stations to be included) in the doass_config configuration script once and to omit the -l option.

$\$$ seed2sac -f $2508375-p$

*It should be noted here, that older rdseed versions store the event depth in kilometres instead of metres. This has been fixed in rdseed 4.6 and it is recommended to use this or a more recent version.

†The "CUSP ID" $\ddagger$ is a 7-digit event reference number assigned by GeoNet. 
The -p option can be used to read phase picks from the SEED file. In case S-phase picks are read from the SEED file, events that contain no pick are not deleted, but stored in the directory SACTRASH/nopicks. They can be deleted manually or kept for manual phase picking. Because of a bug in the SEED files that mislabelled $\mathrm{P}$ and S-phase picks, this function has not been used for the processing of the 2002-2007 data in this study, but can be used for any future work, as the bug has been fixed and phase picks are named properly now. The alternative is to manually retrieve a separate file with the phase picks from GeoNet (phases.txt), compile the readphase.f90 Fortran program, run the program readphase with phases.txt as an argument to extract a .pick file for each event and write the arrival times to the SAC files later (see Appendix A.4).

\$./flush.sh \&\& ./flushseed.sh

SAC data files for stations that are too far away or not found in the station list are collected in a directory SACTRASH (also to be set in the doass_config script). These files can be deleted by executing the script flush.sh after seed2sac. The script flushseed. sh deletes all SEED files that did not contain any usable data.

\section{A.3.1. Prepare data for splitting measurement (I)}

Before the splitting measurements are started, some event information from the info file (depth, magnitude, CUSP ID, distance from Mt. Ruapehu) is stored in the header of the SAC files. For speed and simplicity, only the east component is processed in this step. The scripts are named with the prefix "gns_", because they are optimised for data from the GeoNet network. Phase arrival times are also stored in the SAC file. After this step a SAC file triplet (.e,.n,.z) contains all necessary event information for splitting measurements. Files are finally moved into a directory for each station. Run the following programs without any arguments to display a help message.

\$ gns_info -f 2509957

Looks up the values in 2509957 . info and stores them in all east component files (*.e) from stations that recorded this event.

\$ gns_picks -f 2509957

This program needs to be used only in case the S-picks were read from the SEED file (seed2sac-p). It looks up the phase arrival times in the file $\mathrm{x} 2509957$.picks and stores them in the according east component file (.e) for the station where the phase has been picked.

\$ doass_writephase 2332466

In case the S-phase arrival times have been retrieved in a separate text file, the picks are stored in the SAC file header with the program doass_writephase. This program is called with a filename string (2004.339.FWVZ.2332466) as an argument and reads the pick time from the file 2332466. pick that has been created by the readphase program, where 2332466 is the CUSP ID of the event in this example. 
\$ gns_move -f 2509957

Moves the SAC files of the event with the CUSP ID 2509957 from the directory x2509957 to directories for each station (e.g. events that have been recorded at station WNVZ are moved to a directory WNVZ).

\section{A.4. Processing of data for splitting measurement}

The GeoNet data (or any other data that is in SAC format and contains an S-Pick) can now be processed with the program doass, a shell script that is used to start several other programs and that can process one single event or many events at once. Event data are prepared for splitting measurements and processed with the splitting measurement code. Finally the results are stored in a file and displayed in various ways. The programs that are mentioned in this section can be run without any arguments to display a manual that gives advice for the exact command syntax. A data file triplet is expected to have name suffixes e, $\mathrm{n}$ and $\mathrm{z}$ for east, north and vertical component respectively. Data files in this tutorial are named following a scheme: 2004.001.FWVZ.2165292.e, with year, julian day number, CUSP ID and component.

The following steps will usually be applied to the data from a certain station and the commands are executed from within a stations directory. Note that the seismograms will be cut and the original files are overwritten with these altered shorter ones in the next processing steps. It is advisable to create backups (e.g. \$ tar cvfz wnvz.tar.gz WNVZ/ to create a backup archive of the directory WNVZ). To analyse data from station WNVZ, change to the directory for this station: $\$$ cd WNVZ

\section{Prepare data for splitting measurements (II)}

The shear wave splitting measurement code has some requirements concerning the data files. The data file triplet is required to start at the same absolute time and therefore needs to be synchronised. Broadband data has to be downsampled and long seismograms have to be cut to prevent program crashes.* The S-pick time is saved in the header field $t 5$ of the north component file and events without any S-phase pick will not be included in any further processing. All main scripts used from this step on are named with the prefix "doass_". They should basically work with any data set.

\$ doass_checkspick

Run without any options to display a help message.

\$ doass_checkspick -f 2004.001.FWVZ.2165292

Prepares the SAC files triplet 2004.001.FWVZ.2165292.e, 2004.001.FWVZ.2165292.n

*The maximum length of a seismogram depends on the sampling rate. The cluster analysis program can handle a maximum of 10,000 samples. 
and 2004.001.FWVZ.2165292.z for splitting measurements. The original files will be edited. Backups of the data files should be created before this step.

\section{A.4.1. Find best filter}

To improve the signal to noise ratio and remove unwanted noise from the seismogram, the data can be filtered (See Figure A.1). The program doass_filt -m allows one to quickly apply the same bandpass filter to a data set. It can also be used for an interactive selection of the best filter by visual inspection of the waveforms.

The program doass_findfilt has been developed to automate this task. It applies a set of different filters and ascertains the best by calculating the signal to noise ratio (see next section). The number, type and corner frequencies of the tested filters are set in the macro findfilt.m. By default, fourteen bandpass filters (two-pole one-pass Butterworth) are applied, with corner frequencies of $0.1-1,0.2-2,0.2-3,0.3-3,0.5-4$, $0.6-3,0.8-6,1-3,1-5,1-8,2-3,2-6,3-8$ and $4-10 \mathrm{~Hz}$.

\$ doass_findfilt

Run without any options to display a help message.

\$ doass_findfilt -f 2004.001.FWVZ.2165292

This applies a set of different filters to the event 2004.001.FWVZ.2165292 and selects the three best filters for further analysis. The signal to noise ratio has to be two or greater as a minimum requirement at this point. If no (or less than three) filters result in a file to fulfill this criterion, fewer files will be stored. In the given example, the file triplets 2004.001.FWVZ.2165292.0.3-3.fb1.(enz), 2004.001.FWVZ.2165292. 0.5-4.fb2. (enz) and 2004.001.FWVZ.2165292.0.2-3.fb3. (enz) are created, where the corner frequencies of the bandpass filter are included in the filename and fb1, fb2 and fb3 mark the best, second best and third best filter respectively. This naming scheme allows easy distinction for a later analysis, when only the best filter for each event $(* . f b 1)$, or possibly more than one filter per event (*.fb1, *.fb2, *.fb3) are to be considered.

\section{A.4.2. Calculate signal-to-noise ratio}

The next step is to calculate the signal to noise ratio.* For the shear wave splitting measurements, even a $\mathrm{P}$-wave has to be considered as noise. Therefore, the noiselevel is calculated from a three second window directly preceding the S-pick (-3.05 to $-0.05 \mathrm{~s}$ relative to $\mathrm{S}-\mathrm{pick})$, while the signal is evaluated from a three second window that follows the S-pick (0.05 to $3.05 \mathrm{~s})$. For the signal and for the noise window, the root mean square is calculated (see Section 4.2.2). The ratio is calculated for the east (.e) and north (.n) components and the arithmetic average is used as the signal

\footnotetext{
*The signal to noise ratio has been calculated in the previous step to find the best filters, but in case manually filtered or raw data are analysed the calculation is repeated here. The calculation takes less than half a second for each event on a $1.83 \mathrm{GHz}$ computer.
} 


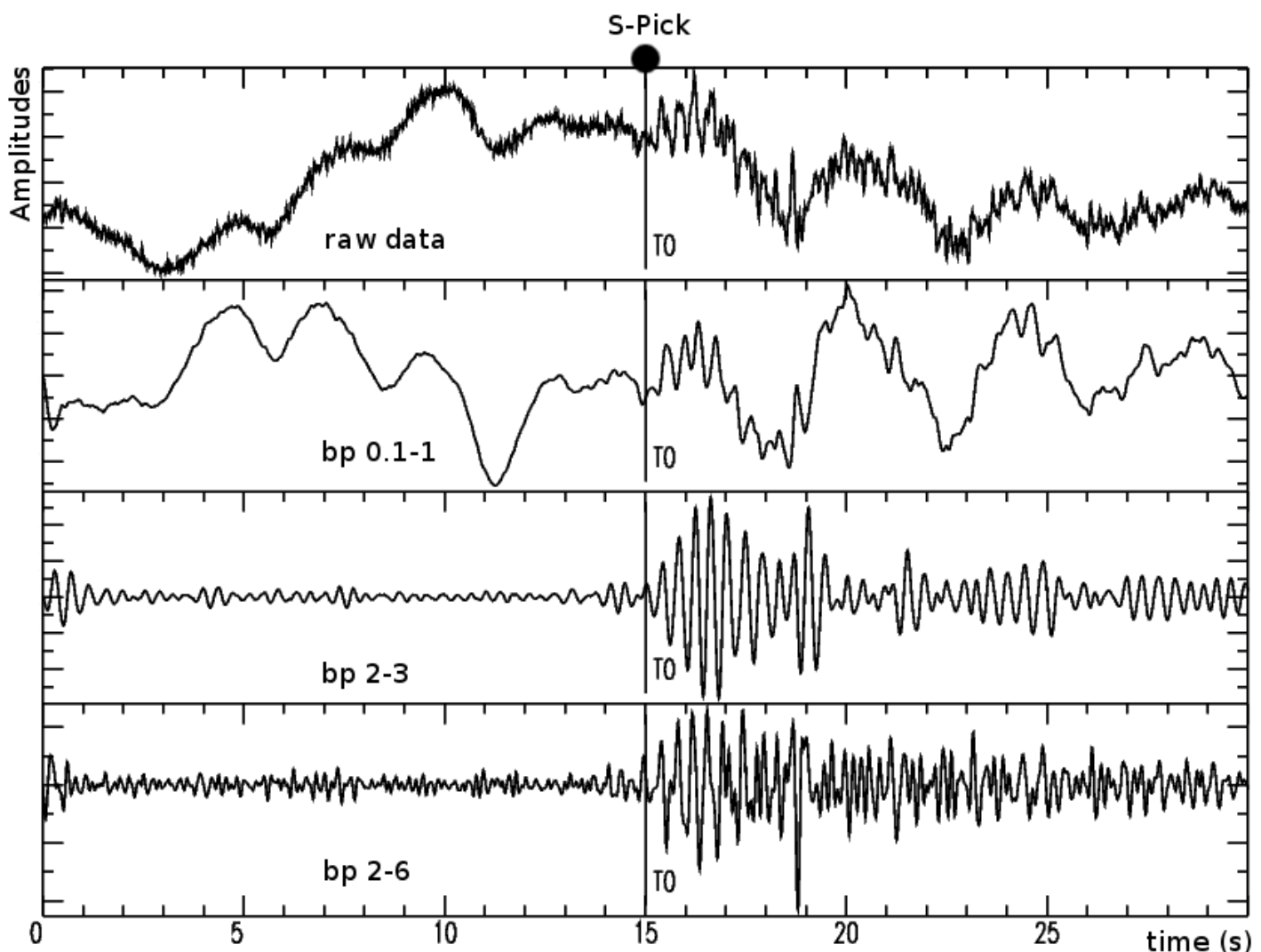

Figure A.1.: Comparison of different filters and their effect on the waveform. Each trace shows a $30 \mathrm{~s}$ window of the east component of event 2002.018.01.08.lqua2 with the S-pick at $15 \mathrm{~s}$. With raw data, the actual signal is hardly visible and no good splitting measurement can be done with this data quality. A bandpass filter with corner frequencies of 0.1 and $1 \mathrm{~Hz}$ does not improve the signal and was automatically rejected by the program. The two bottom traces show the two best bandpass filters for this event, with $2-3$ and $2-6 \mathrm{~Hz}$ corner frequencies. Noise before the phase arrival has been mostly removed and the overall quality of the signal is improved. A comparison of more bandpass filters is shown in Figure 4.3, p. 73. 
to noise ratio for this event.

\$ doass_snr

Run without any options to display a help message.

\$ doass_snr -f 2004.001.FWVZ.2165292

Calculate the signal to noise ratio for 2004.001.FWVZ.2165292.e and 2004.001. FWVZ.2165292.n and write the arithmetic mean of these two values to a file 2004.001.FWVZ.2165292.sn, along with backazimuth and Nyquist frequency.

\section{A.4.3. Create configuration file and measure shear wave splitting}

The Teanby et al. (2004b) shear wave splitting measurement code automatically cycles through a given number of measurement windows (see Sections 4.3.2 and 4.3.3). The number and range of the tested windows is determined by parameters in a configuration file. A general configuration file can either be used for all events or manually created for each single event. The waveforms of the events analysed in this and in the previous studies have a broad variation in terms of frequency content, so that a single configuration setup for all events led to erroneous results. Period lengths of the signal waveform usually vary between 0.7 to $2 \mathrm{~s}$. To address this variability, a customised configuration file can be created for every event before the actual splitting measurement with the program ass (Teanby et al., 2004b) is done. Parameters used for these calculations can be set in the file create_event_ini.sh.

\$ assi -f 2002.016.lhut -s /path/to/file/ass.config.ini

Do a splitting measurement on the event 2002.016. Ihut and use the parameters given in the ass.config.ini file to determine start and end time ranges of the measurement window (Figure A.2), number of measurement windows and other parameters.

\$ assi -f 2002.016. lhut -c

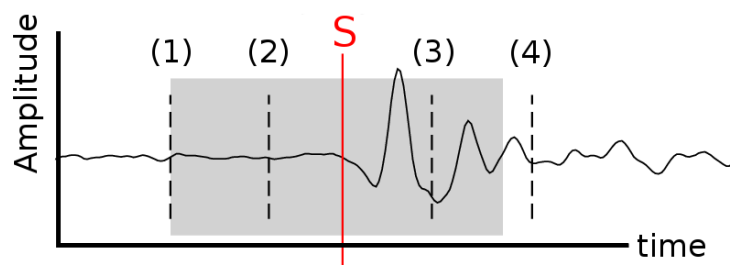

Figure A.2.: Shear wave with measurement window times (1: earliest window start, 2: latest window start, 3: earliest window end, 4: latest window end). The grey box indicates the window that has been chosen after the cluster analysis. 1-4 have been automatically calculated relative to the S-pick (S) by finding the main period length of the S-phase. The plot shows the component parallel to the initial polarisation.

Create an event-specific configuration file and use the parameters in this file for the splitting measurement. The splitting measurement is done with the program ass (Teanby et al., 2004b) and a logfile (.res) is created. The start and end time 
ranges of the measurement windows are calculated with the SAC macro get_T that itself calculates the main frequency $\bar{f}$ of the shear wave. Values of $\bar{f}$ were limited to $0.2 s^{-1} \leq \bar{f} \leq 8 s^{-1}$ to prevent measurement windows that are too long or too short. The main period length is then determined as $\bar{T}=1 / \bar{f}$. An optimal measurement window end range should be from at least half a cycle after the S-pick to a maximum length that does not interfere with neighbouring phases (Teanby et al., 2004b). After the calculation of the main frequency of the shear wave, the minimum and maximum window end times are derived, where the minimum window end time is by default set to $5 / 6 \cdot \bar{T}$ and maximum end time to $2.5 \cdot \bar{T}$. ${ }^{*}$ These values prove to be a good compromise between allowing longer measurement windows that possibly suffer from interfering secondary phases and shorter windows that result in less stable measurements. The number of different window end times $n_{\text {end }}$ depends on the difference between $t_{3}$ and $t_{4}$ and is allowed to vary between 25 and 50 . Window start time limits are set to $t_{2}=t_{S}-0.3$ and $t_{1}=t_{S}-0.7 \mathrm{~s}$ and the number of different window start times is set to $n_{\text {beg }}=3$, because results are less sensitive towards the start time choice (Teanby et al., 2004b). The number of measurement cycles $n$ for one event depends on the number of different window times, where $n=n_{\text {beg }} \cdot n_{\text {end }}$.

\section{Write results into logfile}

Several new files will be created when a splitting measurement is done. The script doass_logfiles is used write results to a logfile. For each measurement, a results file (.res) with relevant event details, measurement parameters and measurement results is created (see Table A.1 for an overview). A description and example content for every field are listed below the table.

1. event: Name of the event and basename of the files. (2006.300.TUVZ.2644522.3-8.fb1)

2. stat: Station name. (TUVZ)

3. slat: Latitude of the station in degrees. (-39.26943)

4. slon: Longitude of the station in degrees. (175.65398)

5. cuspid: A 7-digit event identification number. (2644522)

6. year: Calendar year in which the event occurred. (2006)

7. doy_det: Julian day on which the event occurred, with decimal digits giving the fraction of the day. (300.051)

8. evla: Latitude of the event hypocentre in degrees. (-40.862)

9. evlo: Longitude of the event hypocentre in degrees. (176.915)

10. dist (ev-stat): Distance between event hypocentre and station in $\mathrm{km}$. (206.981)

11. depthkm: Epicentral depth of the event in $\mathrm{km}$. (39.81)

12. mag: Event magnitude. For GeoNet data this is a local magnitude $M_{L}$. $(3.335)$

${ }^{*}$ A small offset of $0.15 \mathrm{~s}$ is added to account for some slightly early S-picks. 


\begin{tabular}{|c|c|c|c|}
\hline 1) & event & 21) & $\delta t[\mathrm{~s}]$ \\
\hline 2) & stat & 22) & $\mathrm{D} \delta t[\mathrm{~s}]$ \\
\hline 3) & slat $\left[^{\circ}\right]$ & 23) & $\Phi\left[^{\circ}\right]$ \\
\hline 4) & slon $\left[{ }^{\circ}\right]$ & 24) & $\mathrm{D} \Phi\left[^{\circ}\right]$ \\
\hline 5) & CUSPID & 25) & anginc $\left[{ }^{\circ}\right]$ \\
\hline 6$)$ & year & 26) & anginc $_{\text {corr }}\left[^{\circ}\right]$ \\
\hline 7) & doy_det & 27) & type_ini \\
\hline 8) & evla $\left[^{\circ}\right]$ & 28) & timestamp \\
\hline 9) & evlo $\left[^{\circ}\right]$ & 29) & comment \\
\hline 10) & $\operatorname{dist}(\mathrm{ev}-\mathrm{stat})[\mathrm{km}]$ & 30) & Nyquist frequency $[\mathrm{Hz}]$ \\
\hline 11) & depth $[\mathrm{km}]$ & 31) & grade $(A, B, C, N, R, u)$ \\
\hline 12) & magnitude & $32)$ & filt $\mathrm{LO}[\mathrm{Hz}]$ \\
\hline 13) & $\mathrm{BAZ}\left[^{\circ}\right]$ & 33) & filt $\mathrm{HI}[\mathrm{Hz}]$ \\
\hline 14) & $\mathrm{S}_{p o l}\left[^{\circ}\right]$ & 34) & $\left|S_{p o l}-\Phi\right|\left[^{\circ}\right]$ \\
\hline 15) & $\mathrm{DS}_{p o l}\left[^{\circ}\right]$ & & \\
\hline 16) & $\mathrm{w}_{b e g}$ & & \\
\hline 17) & $\mathrm{w}_{\text {end }}$ & & \\
\hline 18) & dist_Ruap [km] & & \\
\hline 19) & dist_Ruap [ํ] & & \\
\hline 20) & SNR & & \\
\hline
\end{tabular}

Table A.1.: Fields of results logfile (.res): The comma-delimited logfile contains information about the event and the measurement results. See text for details. A description of all fields is given below.

13. BAZ: Backazimuth in degrees. This is the azimuthal angle at which the signal arrives at the station. (149.087)

14. spol: Initial polarisation of the shear wave in degrees. (41.805)

15. Dspol: Error of $\mathrm{S}_{\text {pol }}$ in degrees, one standard deviation. (1.685)

16. wbeg: Start time of the selected measurement window in seconds, relative to the start of the seismogram at $t=0$ (25.652000)

17. wend: End time of the selected measurement window in seconds, relative to the start of the seismogram at $t=0$ (26.353741)

18. dist_ruap_km: Distance of the event hypocentre from Mt. Ruapehu in kilometres. The distance is given as a surface distance for a spherical earth with a radius $R=6371 \mathrm{~km}$ and is calculated from the point $39.281^{\circ} \mathrm{S} / 175.564^{\circ} \mathrm{E}$ which is located at the centre of Crater Lake. (210.073)

19. dist_ruap_deg: Distance from Mt. Ruapehu in degrees. (1.88924)

20. SNR: Signal to noise ratio for this event. See Section 4.2 .2 for details. (5.43038)

21. tlag: Delay time between fast and slow shear wave in seconds. (0.870000)

22. Dtlag: Error of $\delta t$ in degrees, one standard deviation. (0.028125) 
23. fast: Angle of the orientation of the fast shear wave, in degrees from North. $(-62)$

24. Dfast: Error of $\Phi$ in degrees, one standard deviation. (5.250)

25. anginc: Angle of incidence at the station, measured against a horizontal plane in degrees, where $0^{\circ}$ means vertical incidence. (20.8)

26. anginc_corr: Angle of incidence corrected for topography slope at the area around the station. (20.8)

27. type_ini: Type of measurement. This field contains the measurement code that is used (ass), the number of measurement window start times $n_{\text {beg }}$ and the number of window end times $n_{\text {end }}$. (ass_3_25)

28. timestamp: Date at which the measurement was performed an the logfile (.ilog.ass) was created. (2007-10-12)

29. comment: Any additional information or comment that can be added manually or set in doass_config. (any text)

30. nyquist: Nyquist frequency of the event in Hz. The Nyquist frequency $f_{\text {nyquist }}$ depends on the sampling rate of an event $(f$, in $\mathrm{Hz})$,

where $f_{\text {nyquist }}=f / 2$. (50)

31. gradeABCNR: Evaluation of the measurement quality. (N)

32. filt_LO: Lower corner frequency of the bandpass filter in Hz. (3)

33. filt_HI: Higher corner frequency of the bandpass filter in Hz. (8)

34. spol-fast: Angle between the initial polarisation and the fast orientation in degrees. This value is used to detect null measurements. (76.195)

\$ doass_logfiles -f 2002.016.lhut

This command will create a file 2002.016.lhut.res which contains the fields as described above. This does not need to be done if the measurement has been done with the script assi, in which case the res file is automatically created (see p. 151).

\$ doass_logfiles -a

The -a option will write the res files for all measurements in the current directory. This can be used if some result files were deleted or to assure that a result file for each measurement is present.

\$ doass_logfiles -s

This command is used to create one summary file (.summ) of all measurements in the current directory. It should be used after all events in a directory (e.g. one year's data from one station) have been processed. The summary file contains the same fields as the result file (see Table 4.1) and will have one line per measurement.

\section{A.4.4. Display results}

Measurement results can be illustrated in hodograms (rose diagrams), split maps that show individual measurements as bars on a map, and several other analysis plots. The programs to display these results work in two steps. First, they read out the summary file and select and reject several events according to certain quality 
criteria. Second, they create a PostScript figure that illustrates the results that have passed the selection step.

\$ ass_plot.sh 2002.050.05.34.1hut2.2-3.fb1

This will create a measurement analysis plot (Figure A.3a). This analysis plot is composed of six individual plots and a text box with event information and measurement results. The file containing the analysis plot is named with a prefix all6_, e.g. all6_2002.050.05.34.1hut2.2-3.fb1.ps. The individual plots are also stored as individual files and have the event name (2002.050.05.34.1hut2.2-3.fb1) and a suffix (_wind.eps,_rt.eps,_fs.eps, eventinfo.eps, _error.eps and_clusters.eps). The program only needs the event name as an argument.

$\$$ proses -t 0.8 -f *.summ

Display measurement results of the fast orientation $\Phi$ in a rose diagram (Figure A.3b). A cut-off delay time can be passed to the program (-t option) to reject events with a too high delay time. The program displays six rose diagrams by default. For each deep and shallow events. It plots two rose diagrams of the fast orientation with different quality criteria and a diagram showing null measurements at the bottom. Parameters can be passed on as arguments or can be set directly in the proses script. The program can be run without any arguments to display a help message.

\section{A.5. Automatic measurement and monitoring tool}

To simplify the use of the programs and to keep manual user input to a minimum, the script doass can be used to invoke all commands that are listed in Appendix A.4 above. Starting with a triplet of SAC files, this allows the user the automated processing of one or many events with a single command.

\$ doass -f 2004.002.FWVZ.2165587 -p -r

This command is used for the processing of one event that is passed to the script with the -f option. The data are byteswapped, an S-Pick is stored in the SAC file header, the seismograms are synchronised and cut, the signal to noise ratio is calculated, a configuration file is created, the measurement and the cluster analysis are done and the results are written to a logfile. However, there are some minimum requirements to run all steps. If no S-Pick is found in the file header or the signal to noise ratio is less than 2 , the processing will be interrupted and the user is informed by an error message printed to the terminal.

The next commands will process all files in the current directory by invoking a for loop. This is an easy way to handle large data sets. These commands do not need to be typed manually. They can be copied from the terminal text that is printed to the screen by just invoking doass without any arguments.

for $j$ in $* . e$; do if $[!-f " \$\{j \% . e\} *$.done" $]$; then doass $-p-f \$\{j \% . e\}$; 


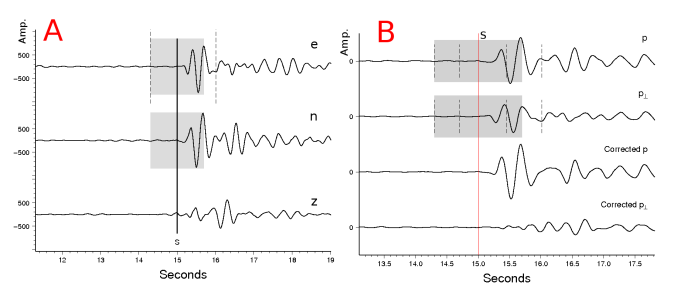

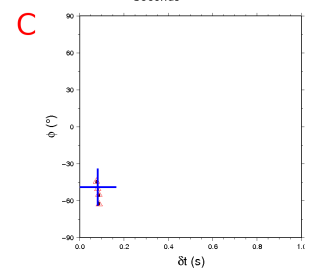

$\mathrm{E}$

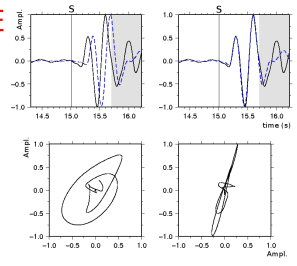

G

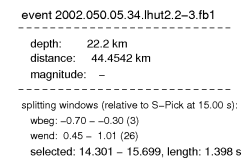

(a) Splitting analysis plot
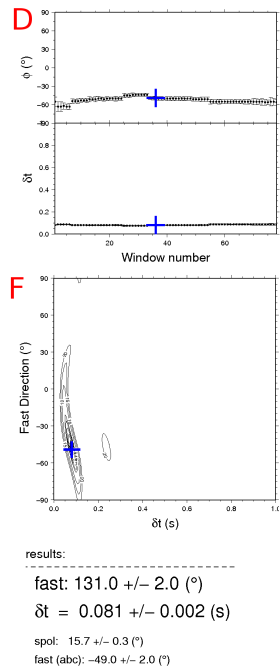
spol: $\left.15.7+1-0.3()^{\circ}\right)$

measurement for event 2002.050.05.34.lhut2.2-3 from the CHARM deployment. See Figure 4.12 (p. 89) for details and a full size figure.
TUVZ 2004-2006

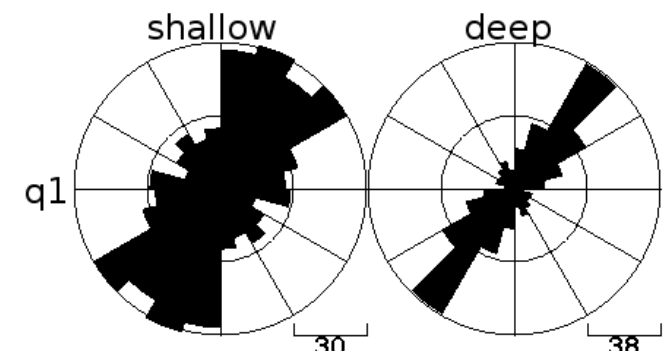

38
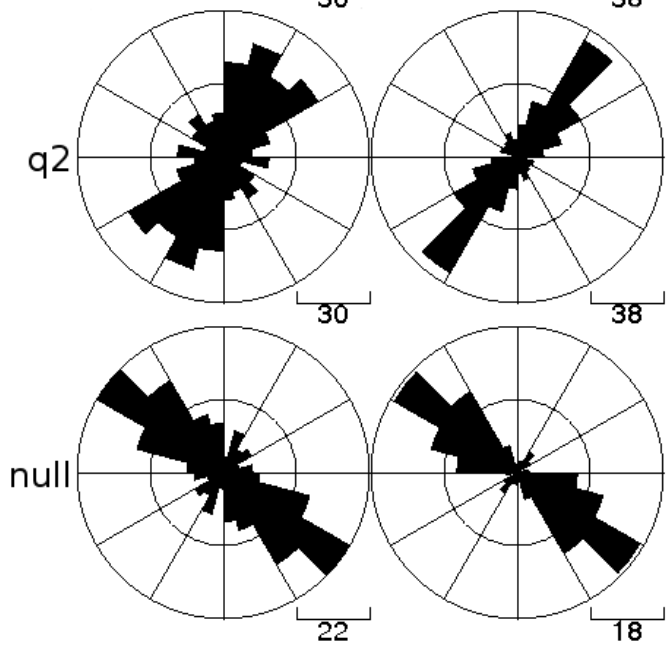

(b) Rose diagram plots. Results from GeoNet station TUVZ for all data from 2004 to 2006. See Figure 4.11 (p. 88) for details.

Figure A.3.:

a) Analysis plot to display results of a single measurement.

b) Rose diagram plots to display trends in the results of several measurements.

\section{fi; done}

Prepare all events for measurement. This script checks for an S-Pick and synchronises the three components. After this step the data are technically ready for a splitting measurement.

for $j$ in $* . e ;$ do if $[!-f$ " $\$\{j \% . e\} *$.done" $]$; then doass $-x \quad 9-f$ $\$\{j \% . e\} ; f i$; done

This command invokes the program doass_findfilt and automatically selects up to three bandpass filters that give the best signal to noise ratio for each event. This is an optional step, but raw data rarely give any good results when local events are analysed.

for $j$ in $* . f b 1 . e ;$ do if $[!-f " \$\{j \% . e\} *$.done" $]$; then doass $-r-f$ $\$\{j \% . e\} ; f i$; done 
All events for which a sufficiently good filter has been found are now analysed for shear wave splitting. The for loop now includes the events with the best filter that was found $(* . \mathrm{fb} 1 . e)$. For sparse data sets it is possible to include also the secondand third best filter for each event by adding $*$.fb2.e and/or *.fb3.e to the for loop.

All commands can be entered at once to process all files in the current directory in a single step:

for $j$ in $* . e$; do if [ ! -f $" \$\{j \% . e\} *$.done" ]; then doass $-p-f \$\{j \% . e\}$; $f i$; done; for $j$ in $* . e$; do doass $-x 9-f \$\{j \% . e\}$; done; for $j$ in

$* . f b 1 . e ;$ do if [ ! -f $" \$\{j \% . e\} *$.done" ]; then doass $-r-f \$\{j \% . e\}$;

$\mathrm{fi}$; done; doass_logfiles $-\mathrm{s}$; proses -t 0.8 -f *.summ

Starting with a directory containing several SAC file triplets, these commands find the best bandpass filters for each event, perform the measurements, write a summary logfile and display the results in a rose diagram (proses). Again, this command line does not need to be typed by the user. By running doass without any options, some commonly used command lines are printed to the terminal and can simply be copied. A "Cheatsheet" at the end of this Appendix gives a quick overview of all commands and programs.

\section{A.6. 3rd party software used for this study}

Essential parts of this work have been done with the help of the following software and tools:*

Interpreters: Bash, $\mathrm{Csh}^{\dagger}$

SAC 100.1 Seismic analysis code was used to load, plot, filter, edit and process the seismograms. See Goldstein and Snoke (2005).

GMT 4.2.0 Most of the plots and almost all of the maps were created with the Generic Mapping Tools developed by Wessel and Smith (1991).

Teanby cluster analysis code The cluster analysis code developed and published by Teanby et al. (2004b) constituted the core of the programs that have been used. During the project, source code and scripts have been modified to adjust to the requirements of this specific study. The code was compiled with the free compiler g77. For a more detailed description of this code see Section 4.3.3.

g77 Fortran compiler The GNU project Fortran 77 compiler was used to compile the cluster analysis code.

${ }^{*}$ Some of the programs require certain environment variables to be set. Further information can be found in the according Readme and manpages.

$\dagger$ Although the shell scripts have been mainly developed following Bash syntax, several GMT scripts use the $\mathrm{C}$ shell as an interpreter and not all of them were rewritten into Bash syntax. As Bash and Csh are both freely available, it is recommended to have both set up when running the scripts. The programs can be started from a Bash or Csh environment, because the scripts select their correct interpreter with a special command in the first line (e.g.\#!/bin/bash). 
f95 Fortran compiler The GNU project Fortran 95 compiler was used to compile the readphase.f90 program that reads S- and P-picks from a GNS phases file.

Unix tools: cat, awk, sed, grep and many more.

\section{A.7. Cheatsheet}

The next two pages contain a short guide to use the automatic shear wave splitting measurement programs and can be printed or copied for reference. 
get data

- select events

- GeoNet earthquake search* or internally from GeoNet system

- other sources

- get SEED file

- GeoNet FTP server ${ }^{\dagger}$

- S-picks from seed files or separately

- extract SAC file

- delete data from stations that are not of interest

- delete non-3-component seismograms

- archive SEED file / delete if it contains nothing useful

- store event info in SAC file (magnitude, depth, CUSP ID,)

- move events to a separate directory for each station

preparation (in station directory)

- byteswap data (if extracted on another machine)

- write the S-phase picks into the files ${ }^{\ddagger}$

- check S-pick

- synchronise all three components, remove mean and trend, cut seismograms, downsample or cut further if still too many samples

- apply filter

- raw data

- one filter for all data

- choose best filter interactively

- find best filter automatically

- calculate signal to noise ratio (SNR)

splitting measurements and cluster analysis (time- and CPU-intensive)

- create an event-specific ass.ini configuration file (considers different window ranges according to the period length of the event; selects a reasonable number of windows and step length)

\footnotetext{
*http://magma.geonet.org.nz/resources/quakesearch/

$\dagger$ ftp.geonet.org.nz/cusp

${ }^{\ddagger}$ phases from cusp system; if no S-pick has been picked, the P-pick is used to calculate the S arrival later $(\mathrm{vp} / \mathrm{vs}=\mathrm{sqr} 3)$
} 
- write logfiles (.res, .summ)

- reads all information from several files

- creates a .res result file for each event

- creates a .summ summary file for each station

\section{further analysis}

- quality control (signal to noise ratio, maximum delay time, maximum error for $\Phi$, stable windows, null measurements)

- analysis plots (all6_.....) for visual quality control (e.g. linearity of the particle motion) of single measurements

- rose diagrams (for each station)

- splitting on a map, density plots of parameters vs depth (to look for spatial changes)

- splitting parameters vs time (to look for temporal changes)

\section{Quick command guide:}

Starting with SAC file triplets which have S-phase picks stored in the header field To, the following commands can be used to automatically do shear wave splitting measurements with all events in the current directory. These commands can be copied from the screen when invoking the command doass without any options.

- Prepare:

for $j$ in $* . e ;$ do if $[!-f " \$\{j \% . e\} *$.done" $]$; then doass $-p-f$ $\$\{j \% . e\} ; f i ;$ done

- Filter:

for $j$ in $* . e ;$ do if $[!-f$ " $\$\{j \% . e\} *$ done" $]$; then doass $-x \quad 9-f$ $\$\{j \% . e\} ; f i ;$ done

- Measurement:

for $j$ in *.fb1.e; do if $[!-f " \$\{j \% . e\} *$.done" $]$; then doass $-r$ -f $\$\{j \% . e\}$; fi; done

- Further analysis:

doass_logfiles -s; proses -t $0.8-f *$.summ

- All in one step:

for $j$ in $* . e ;$ do if $[!-f " \$\{j \% . e\} *$.done" $]$; then doass $-p-f$ $\$\{j \% . e\} ; f i$; done; for $j$ in $* . e ;$ do doass $-x 9-f \$\{j \% . e\}$; done; for $j$ in $* . f b 1 . e$; do if $[!-f$ " $\$\{j \% . e\} *$.done" $]$; then doass $-r$ -f $\$\{j \% . e\}$; fi; done; doass_logfiles $-s$; proses -t $0.8-f *$. summ 


\section{B. Properties of the seismic data}

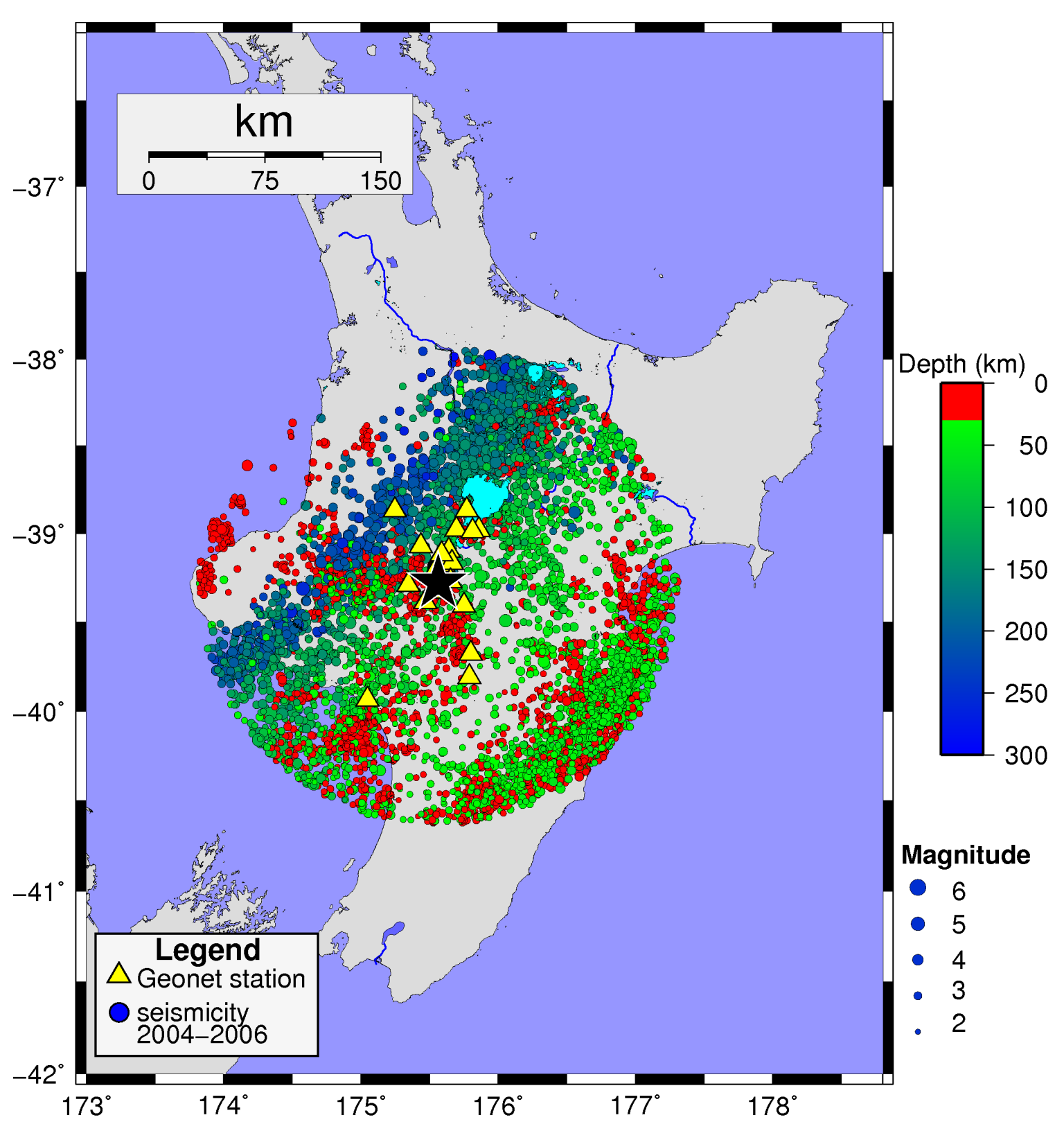

Figure B.1.: Selected events in 2004-2006 with a hypocentral distance of up to $150 \mathrm{~km}$ from Ruapehu and a magnitude of 2 or greater. 8502 events passed this selection for this 3-year time period. Separate maps for the selected shallow and deep events are shown in Figure 5.3, p. 96. 


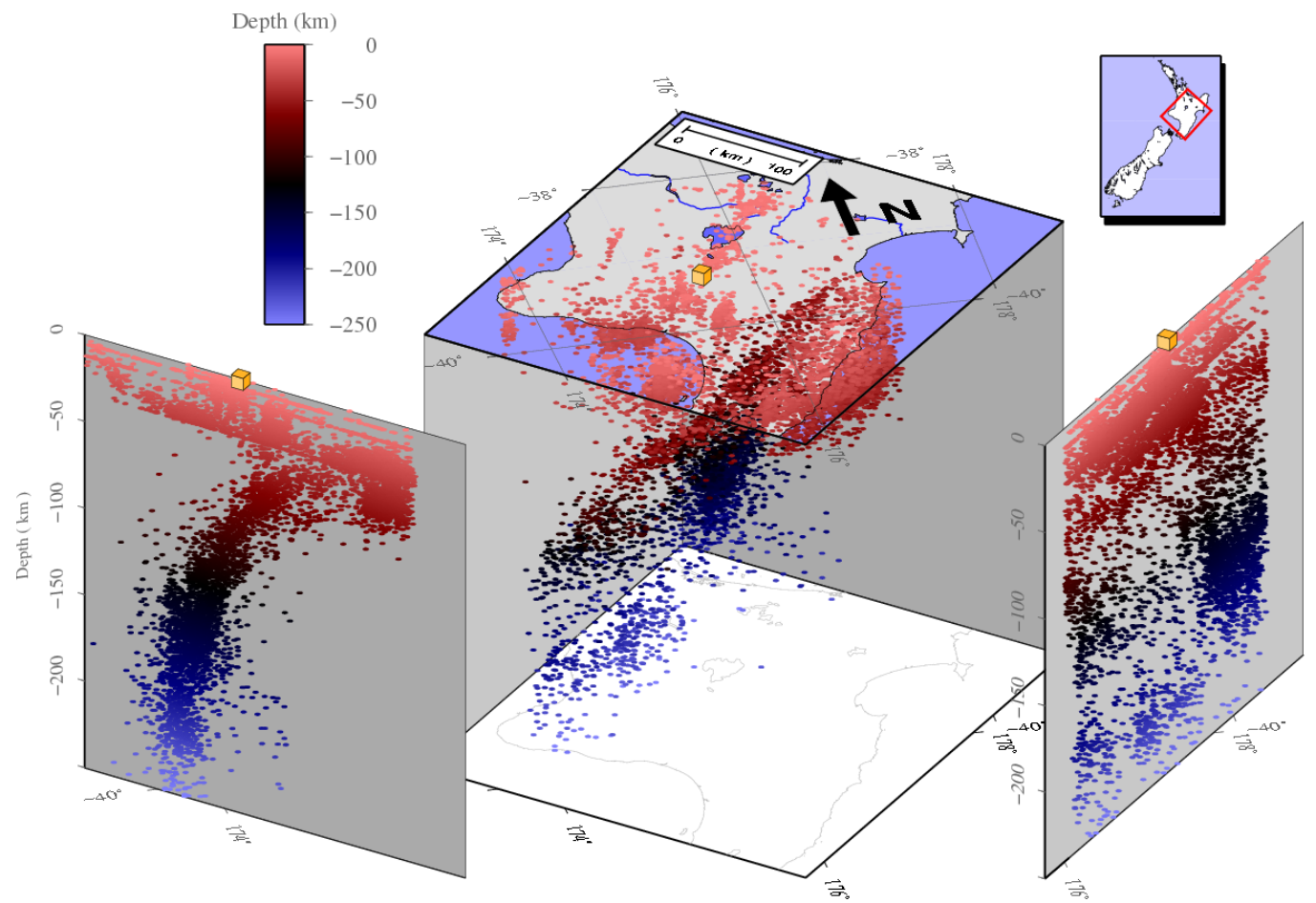

Figure B.2.: 3D projection of seismicity on the North Island. Only events with a magnitude of two or greater that occurred 2004-2006 are shown (the same selected events as in Figure B.1. The hypocentres of deep events indicate the shape of the subducted slab of the Pacific plate in the NW-SE profile view (left). The position of Mt. Ruapehu is marked with a cube. Comparison with a similar plot for CHARM data from 2002 (Figure 4.4 of Gerst, 2003) suggests that the source locations of seismicity have not experienced major spatial changes. 


\section{Measurement results displayed on split maps}

All split maps show single measurements represented by bars on a map view. The bars in the following maps are plotted at the surface projection of the point where a wave would pass at $50 \mathrm{~km}$ depth. This does not mean that the ray path really travelled through the marked spot at a depth of $50 \mathrm{~km}$ (the projection is based on the arrival angle at the station, but near-surface low-velocity layers cause steep arrival angles). Neither do these figures imply that the shear wave splitting occurred at the point of the bar. Illustrating the results in these split maps can be used to investigate spatial trends in the data.

\section{C.1. Yearly data sets, all stations}

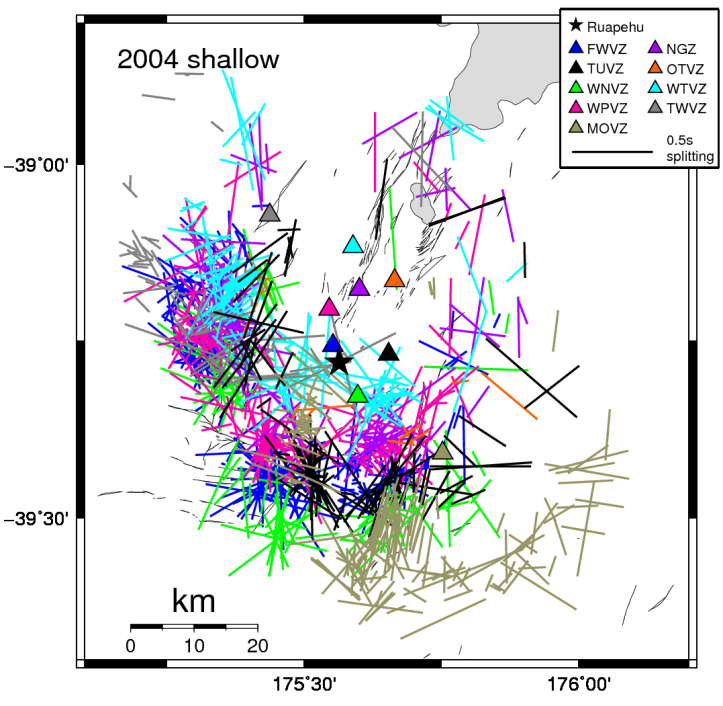

(a) shallow

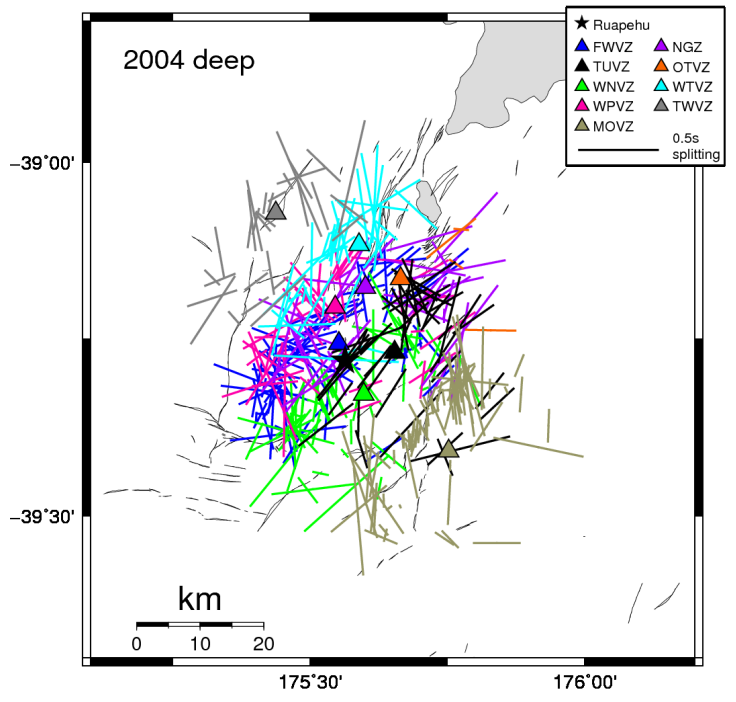

(b) deep

Figure C.1.: Results from 2004 data. All splitting measurement in this and the following figures are plotted at the projected point where the ray path pierces a depth of $50 \mathrm{~km}$. 


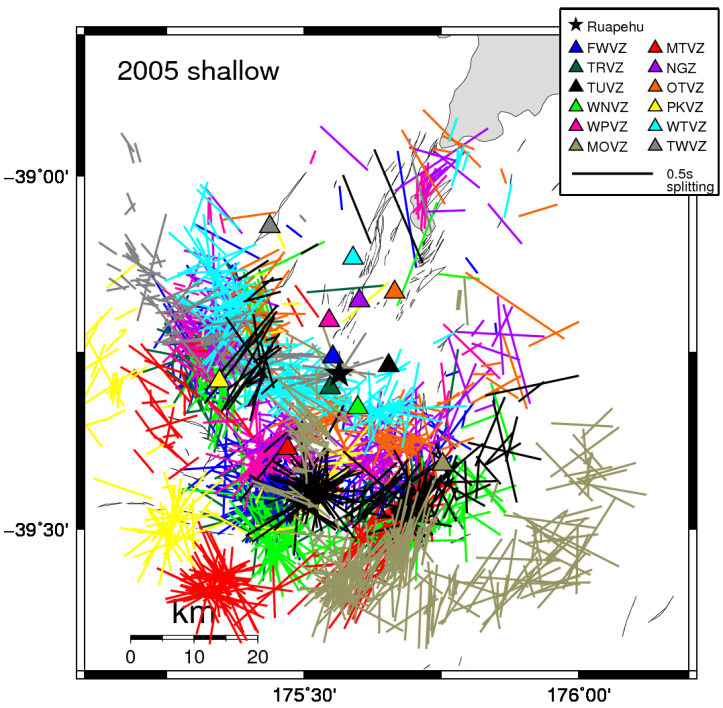

(a) shallow

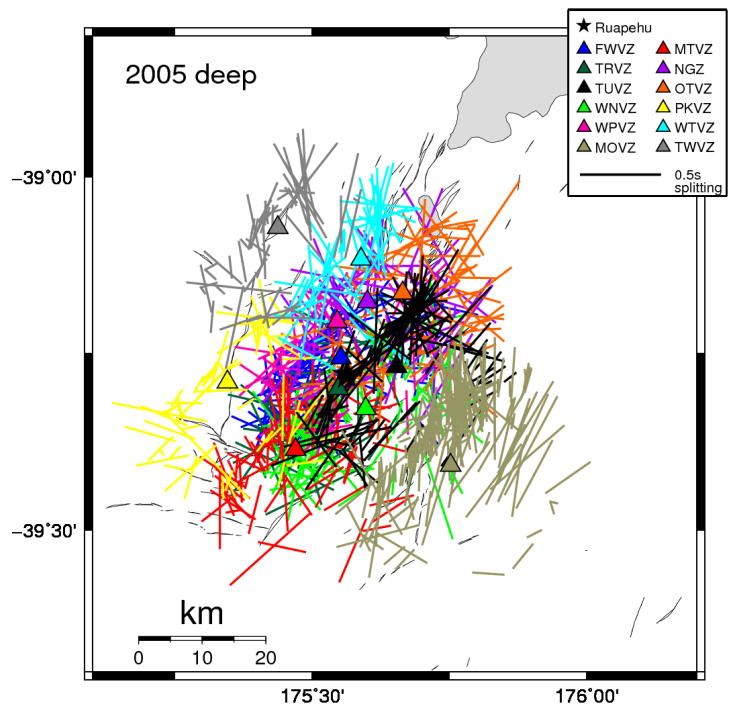

(b) deep

Figure C.2.: Results from 2005 data

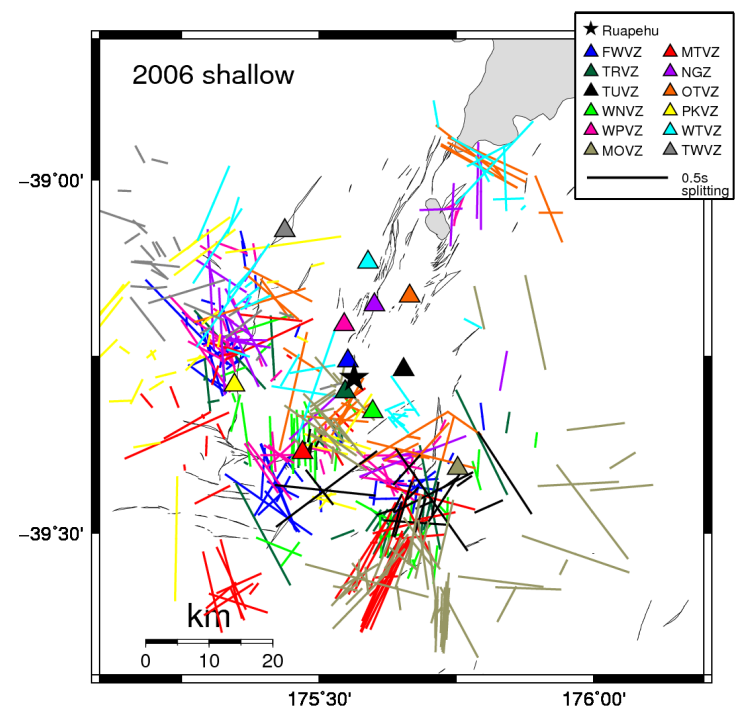

(a) shallow

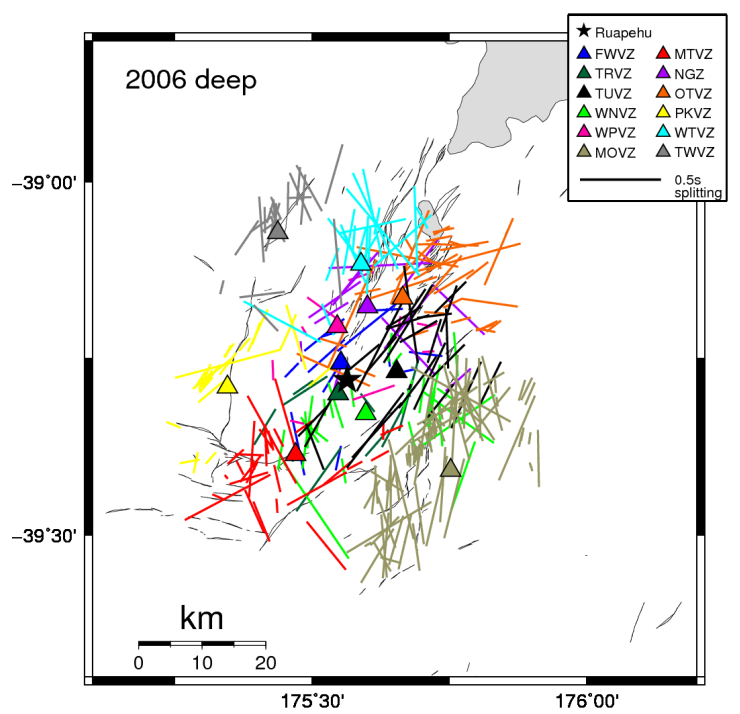

(b) deep

Figure C.3.: Results from 2006 data 


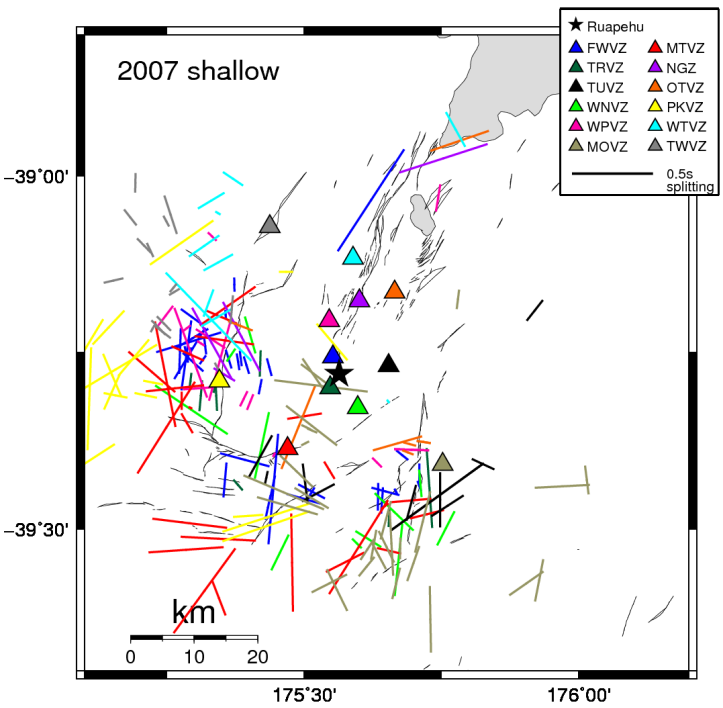

(a) shallow

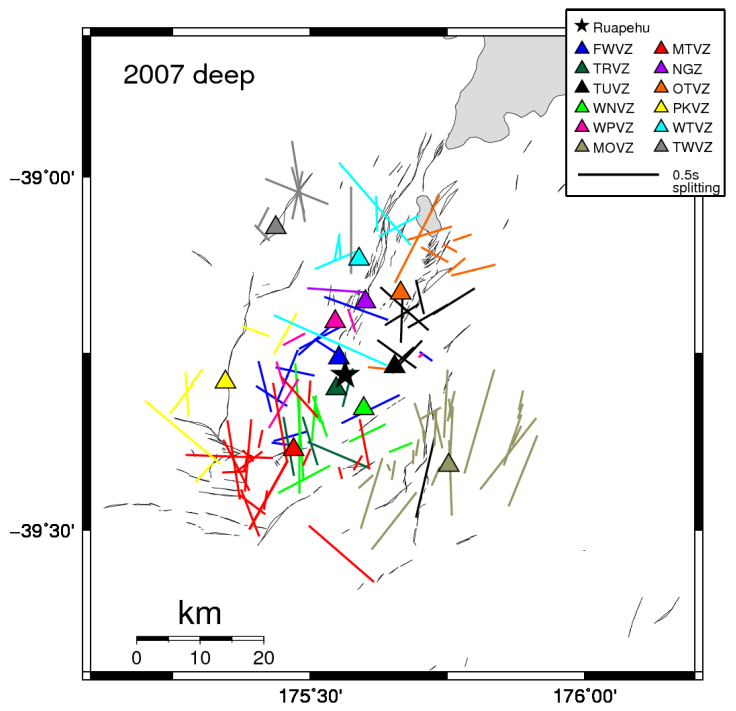

(b) deep

Figure C.4.: Results from 2007 data

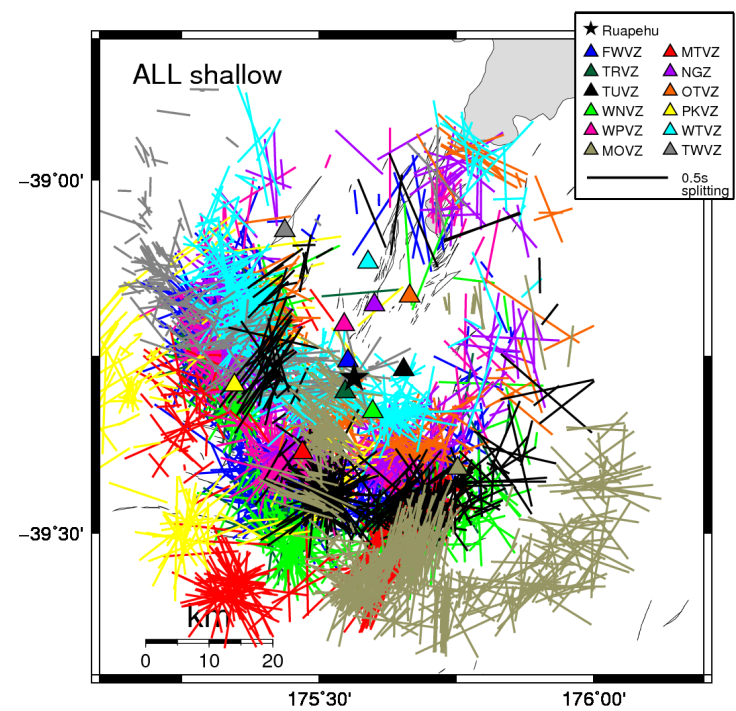

(a) shallow

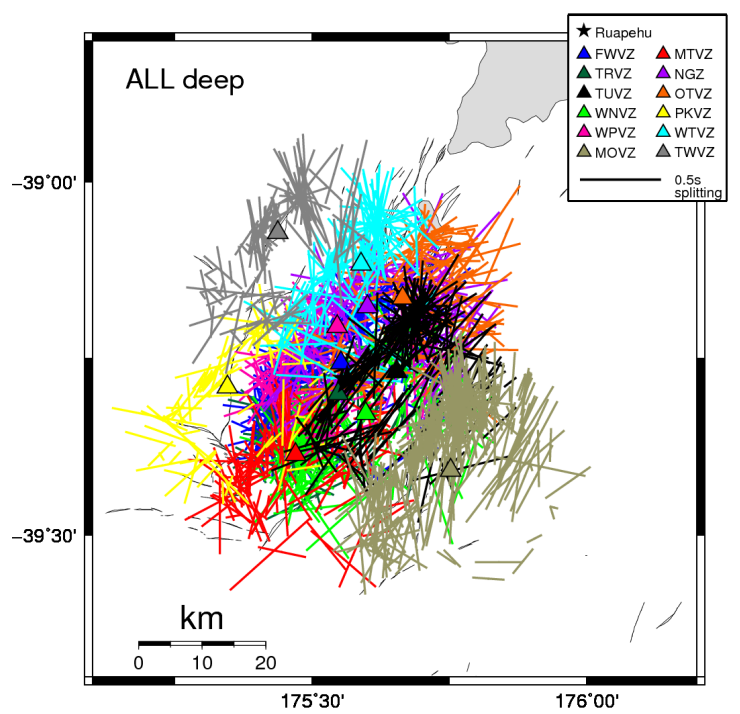

(b) deep

Figure C.5.: Results from 2004-2007 data 


\section{C.2. Results of single stations}

Results are shown below on separate maps for each station. Every map shows all measurements obtained at the station in 2004-2007.

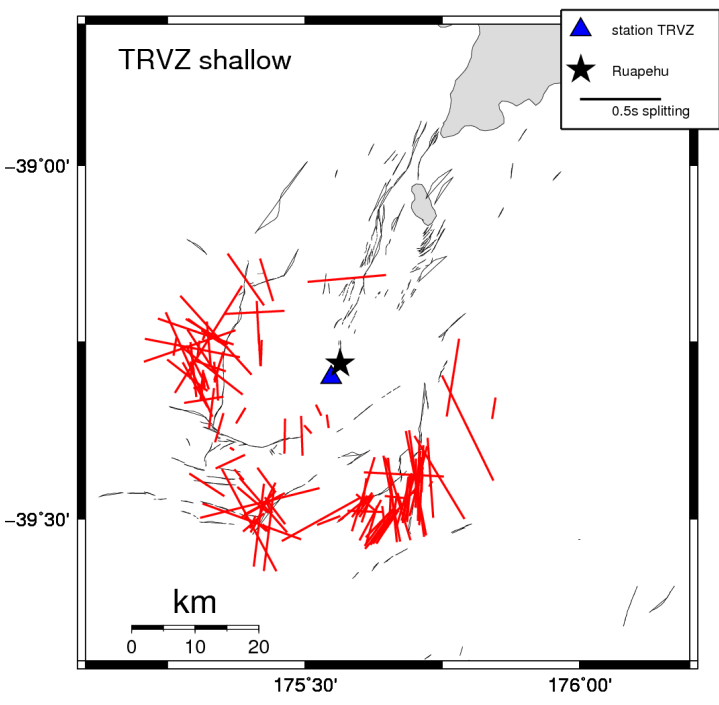

(a) shallow

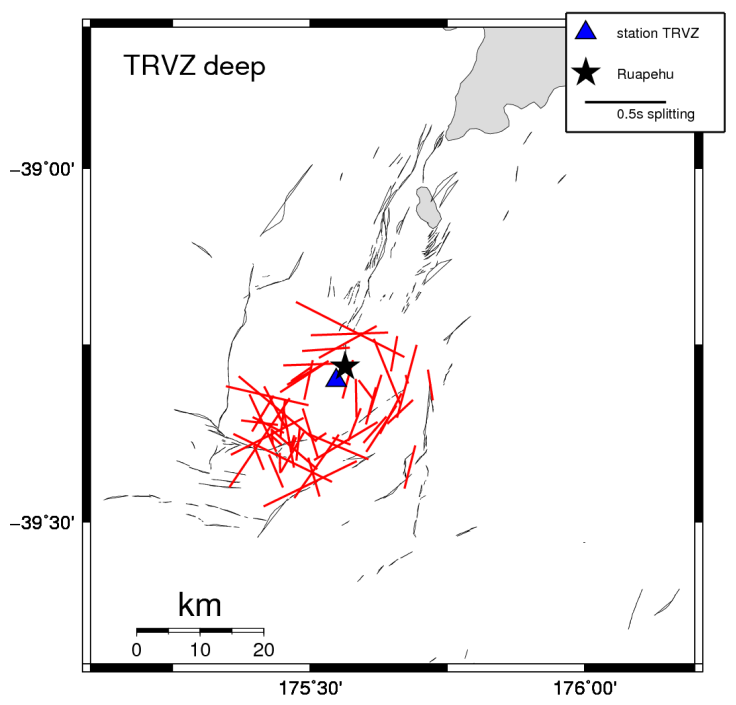

(b) deep

Figure C.6.: Results from station TRVZ

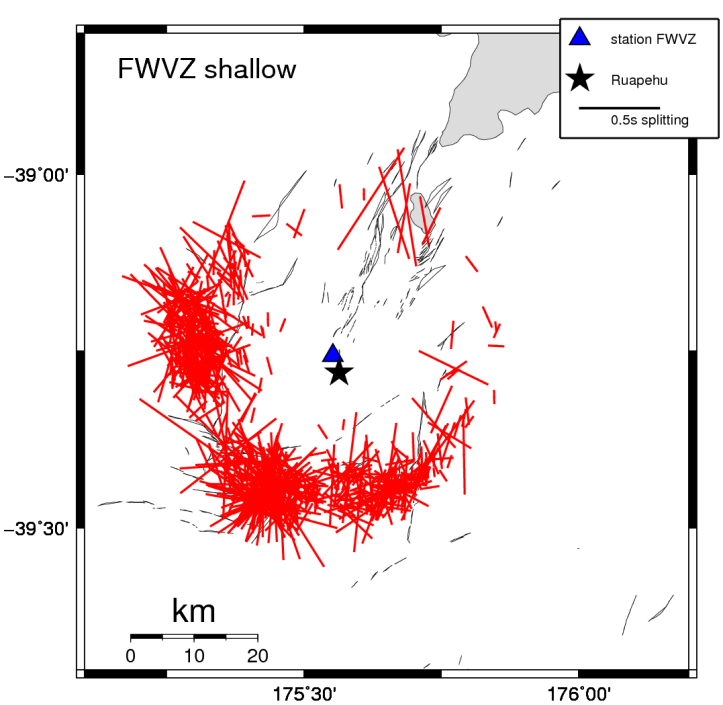

(a) shallow

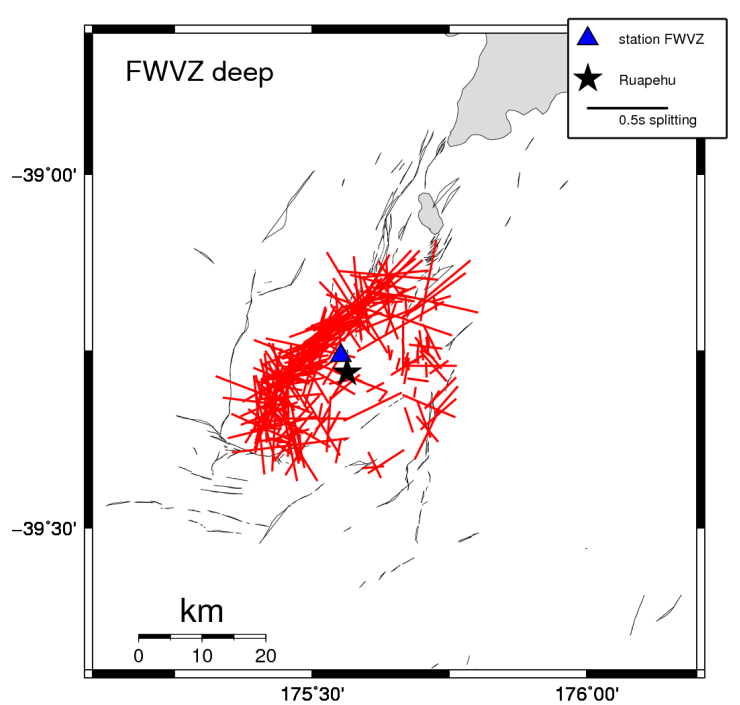

(b) deep

Figure C.7.: Results from station FWVZ 


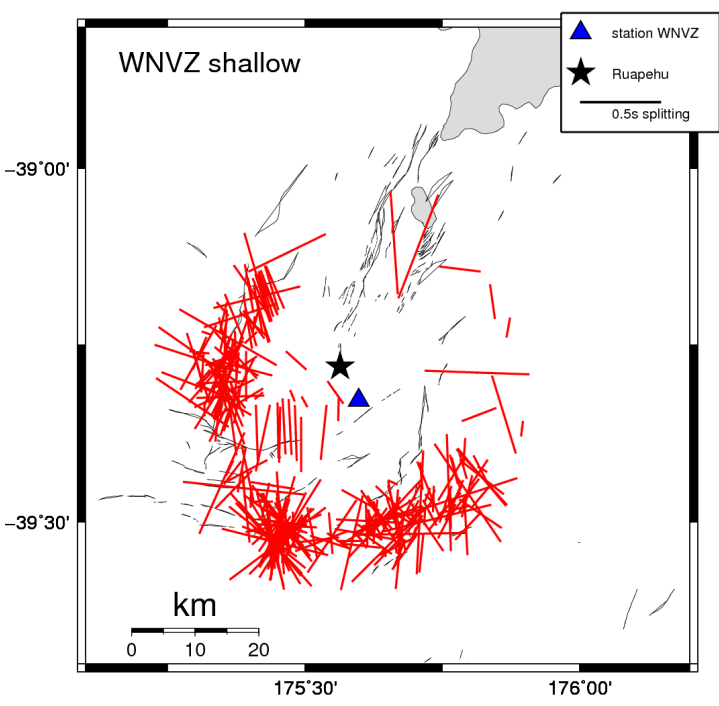

(a) shallow

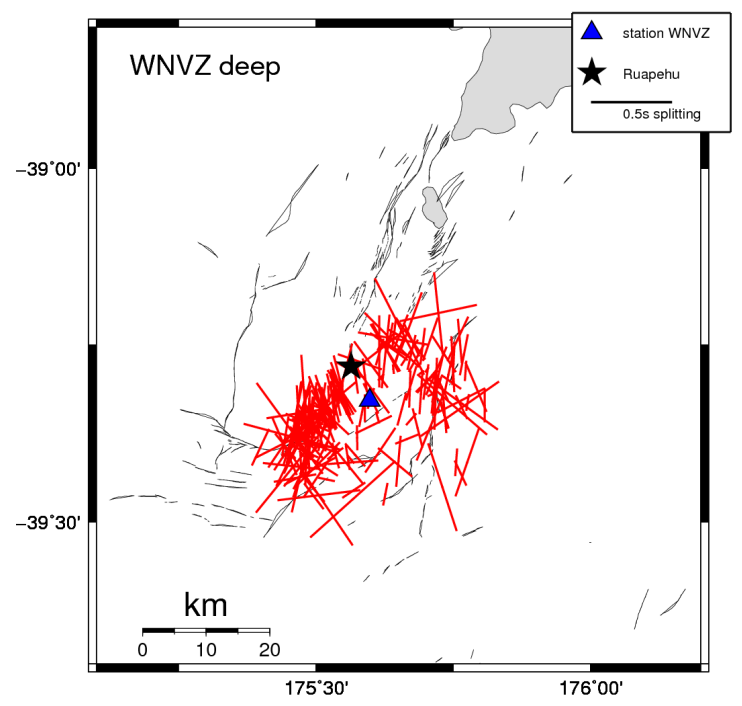

(b) deep

Figure C.8.: Results from station WNVZ

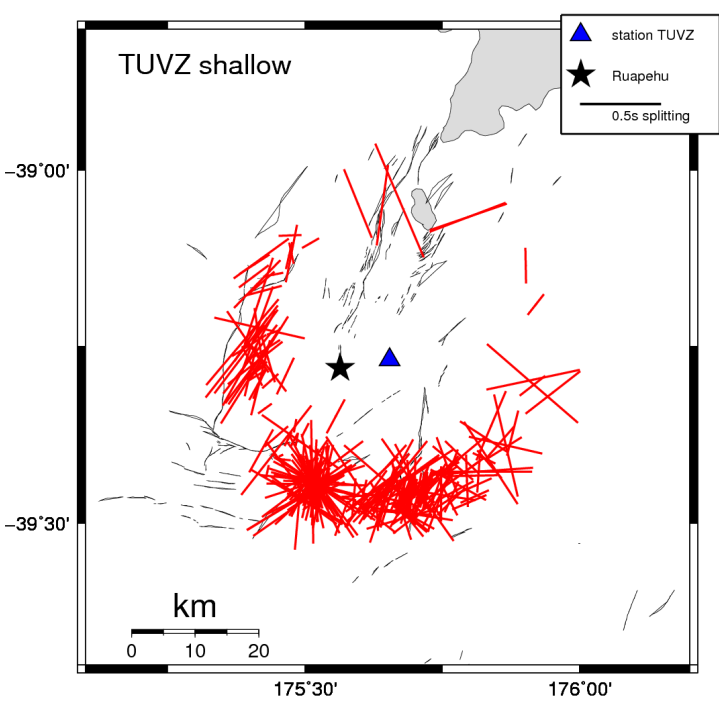

(a) shallow

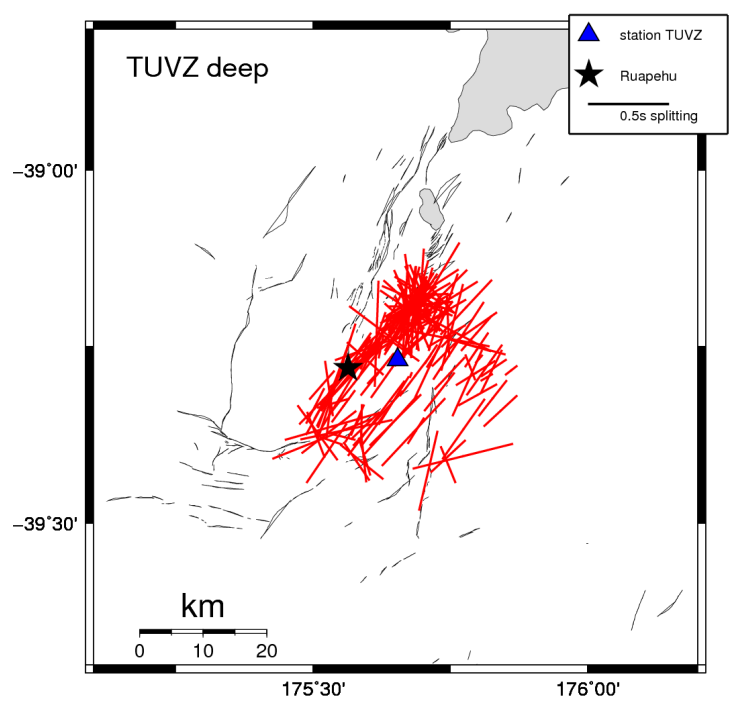

(b) deep

Figure C.9.: Results from station TUVZ 


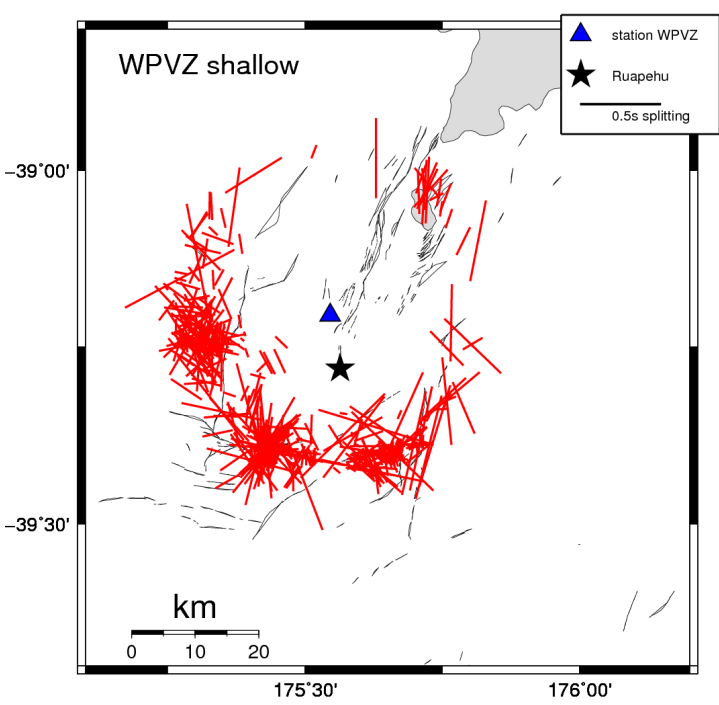

(a) shallow

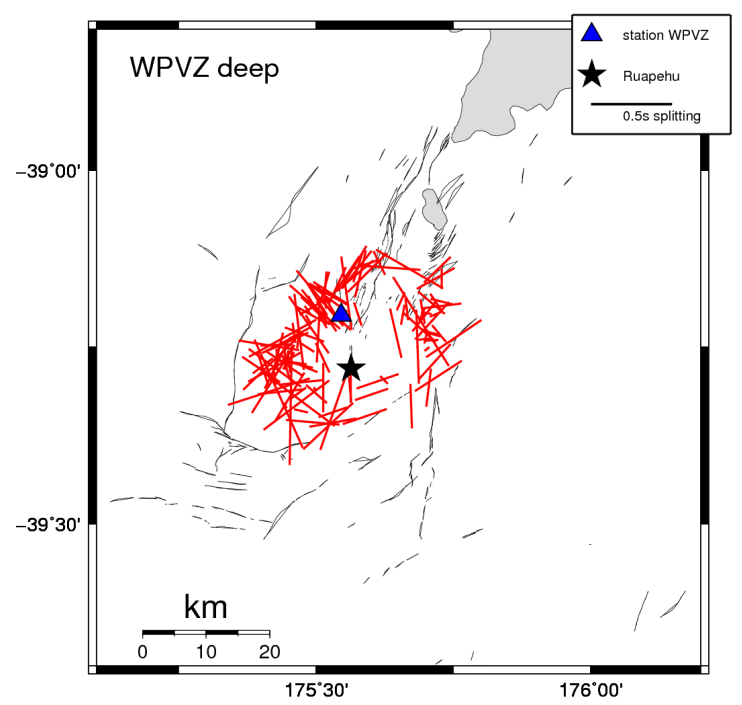

(b) deep

Figure C.10.: Results from station WPVZ

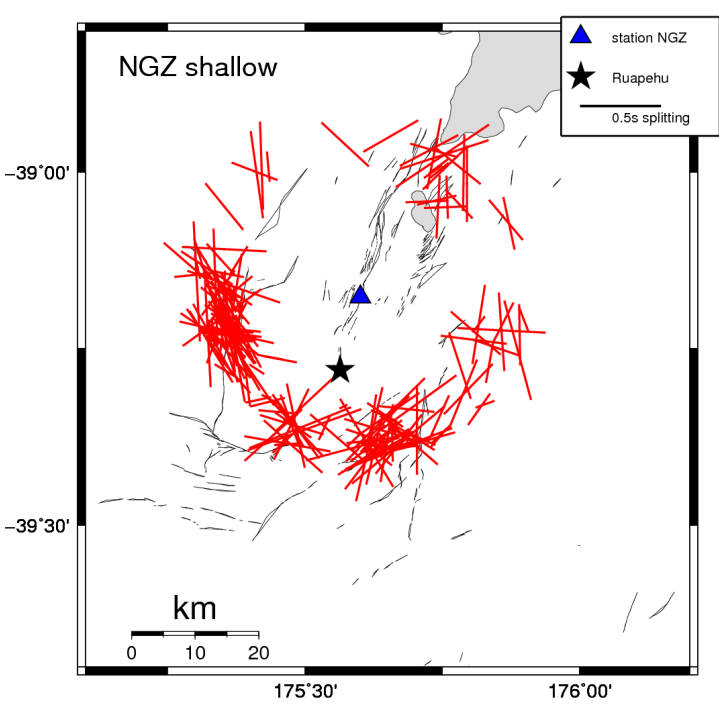

(a) shallow

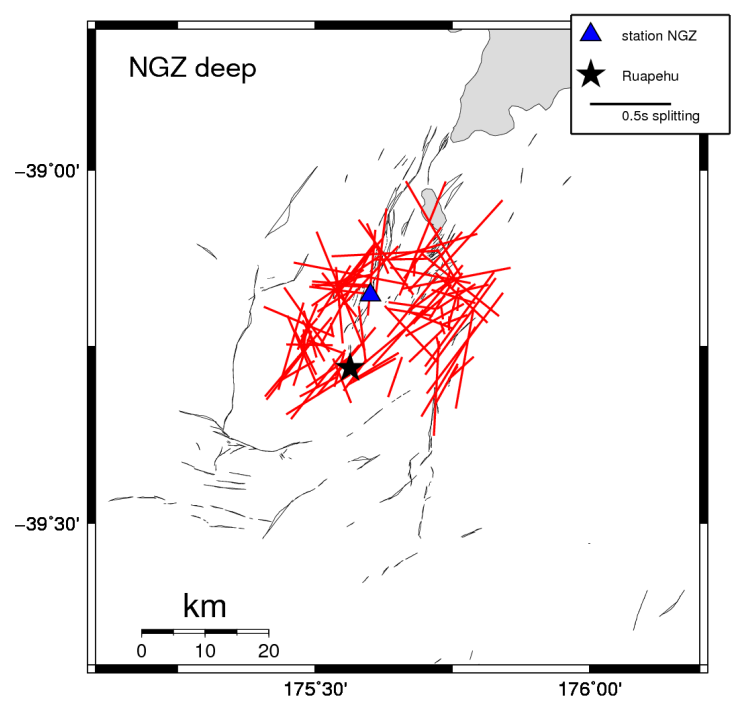

(b) deep

Figure C.11.: Results from station NGZ 


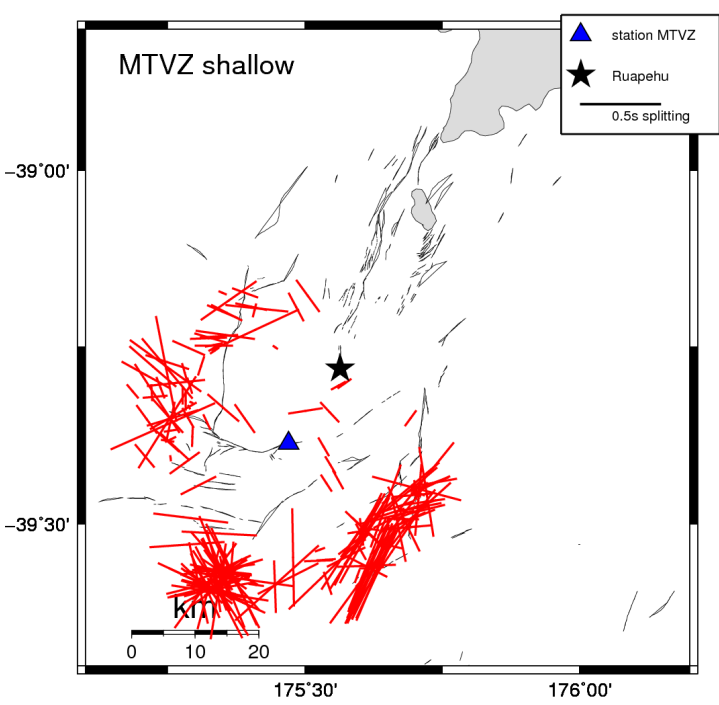

(a) shallow

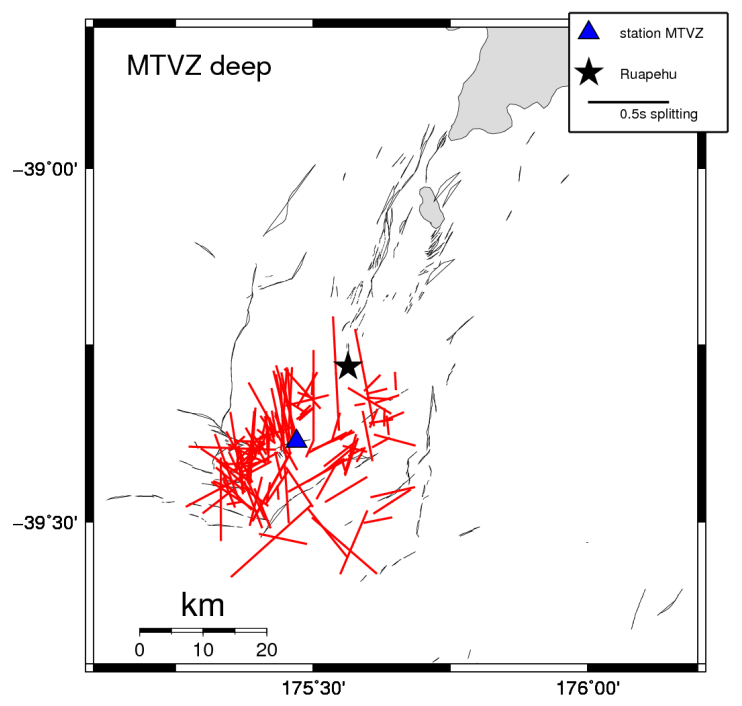

(b) deep

Figure C.12.: Results from station MTVZ

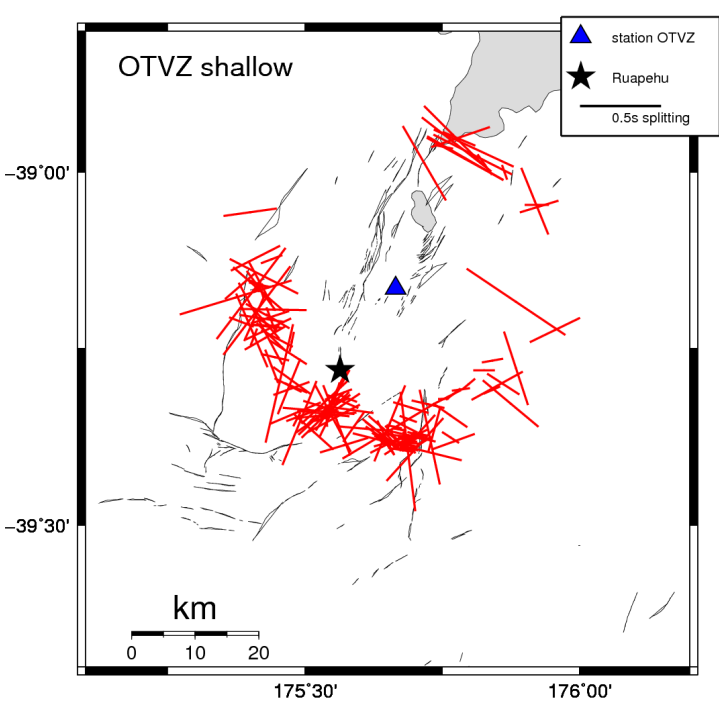

(a) shallow

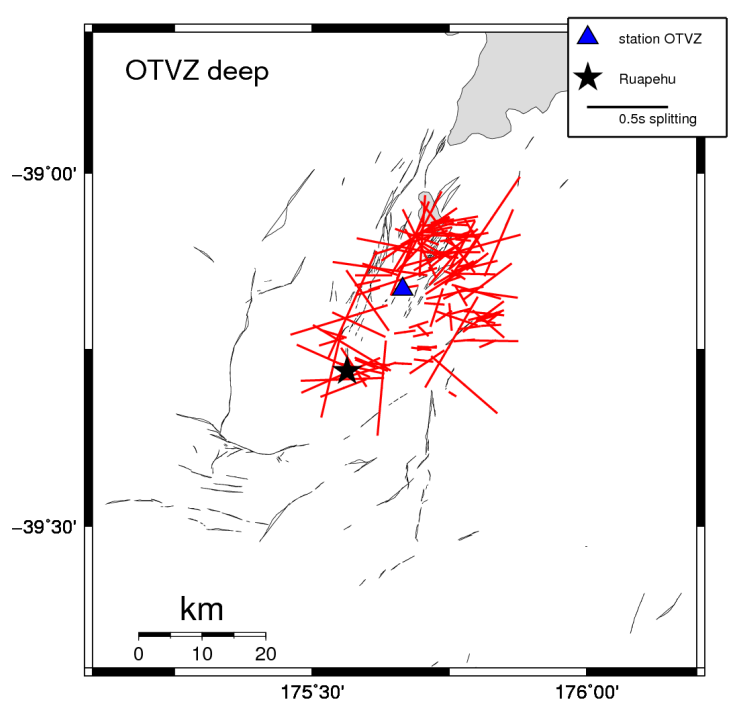

(b) deep

Figure C.13.: Results from station OTVZ 


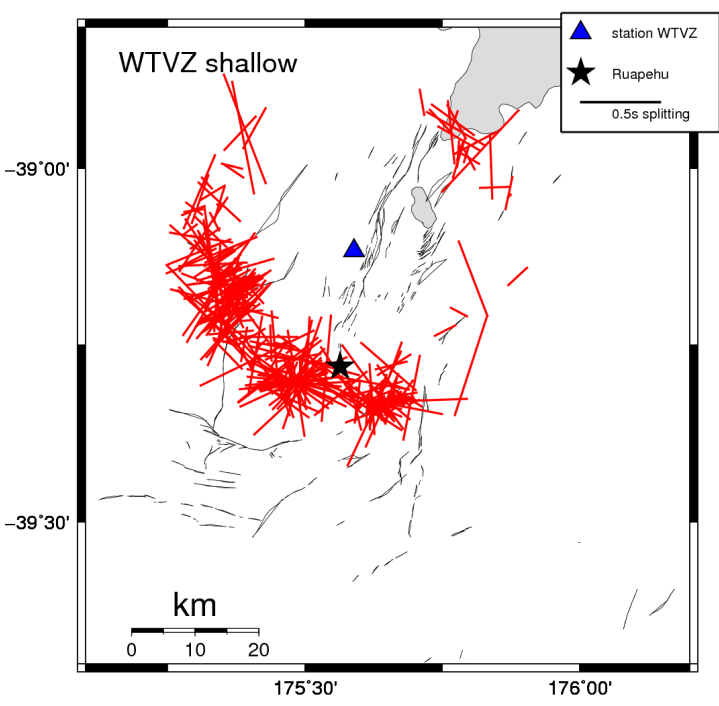

(a) shallow

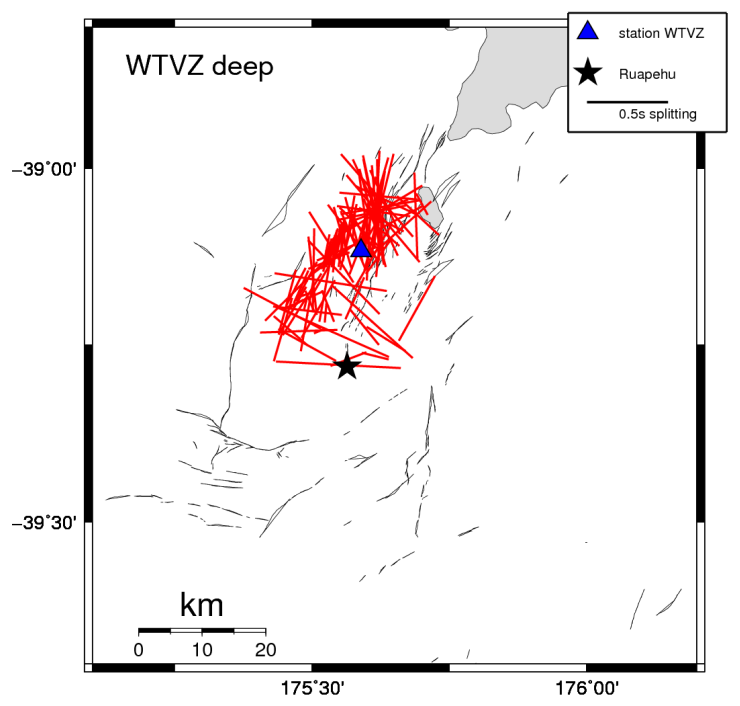

(b) deep

Figure C.14.: Results from station WTVZ

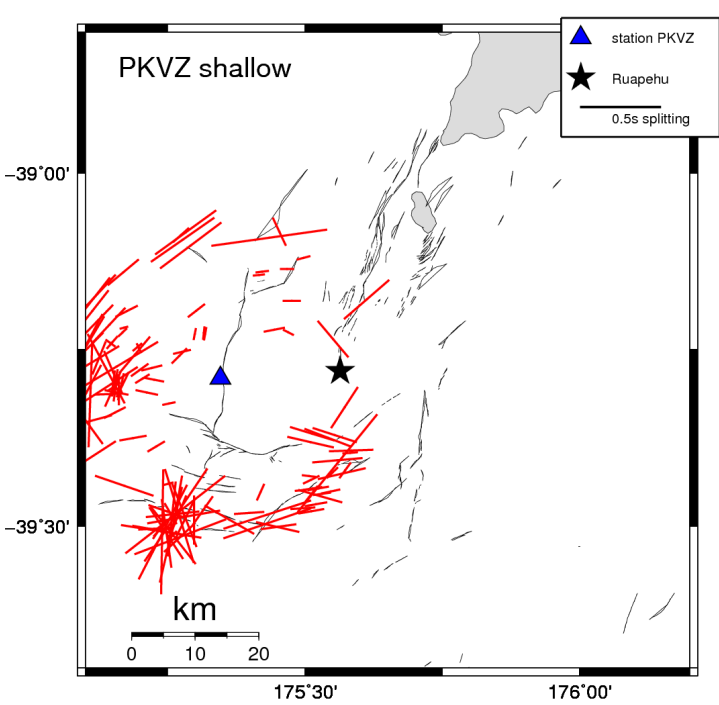

(a) shallow

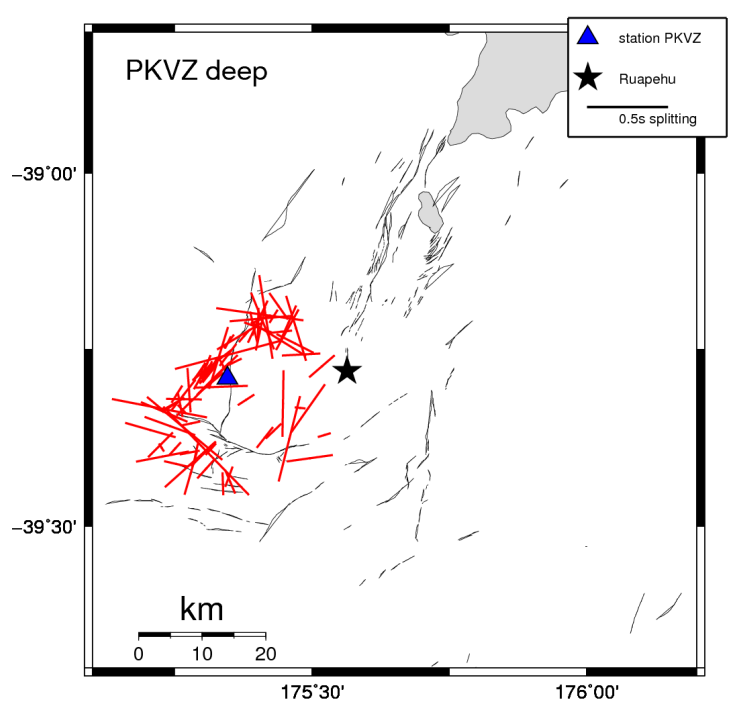

(b) deep

Figure C.15.: Results from station PKVZ 


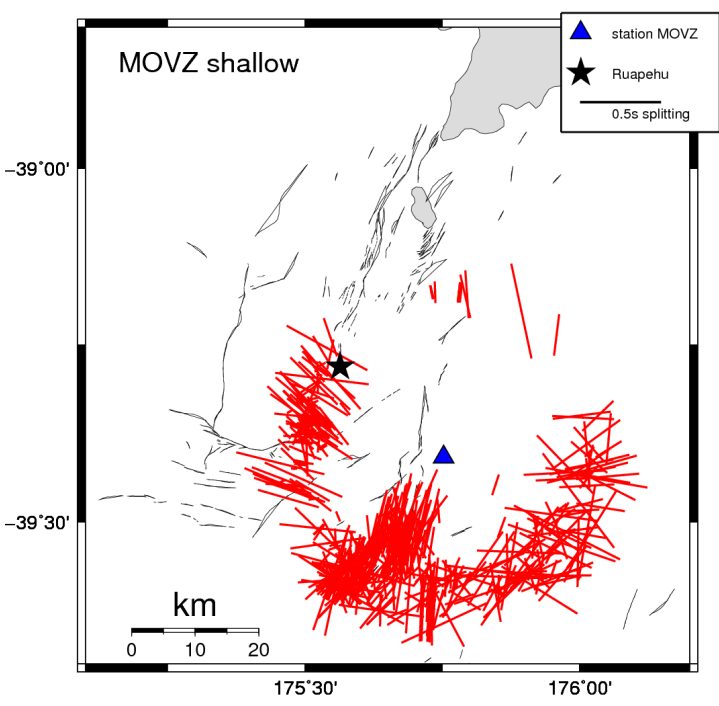

(a) shallow

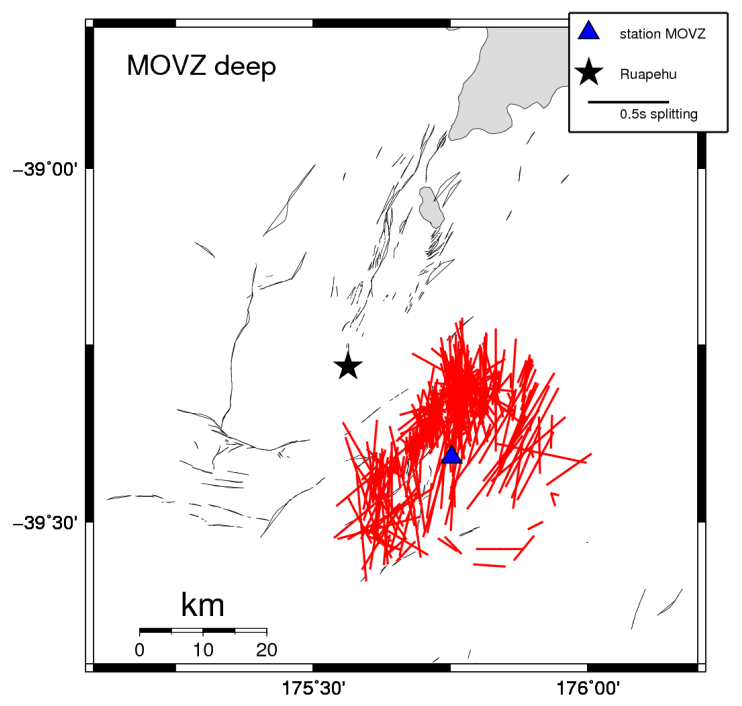

(b) deep

Figure C.16.: Results from station MOVZ

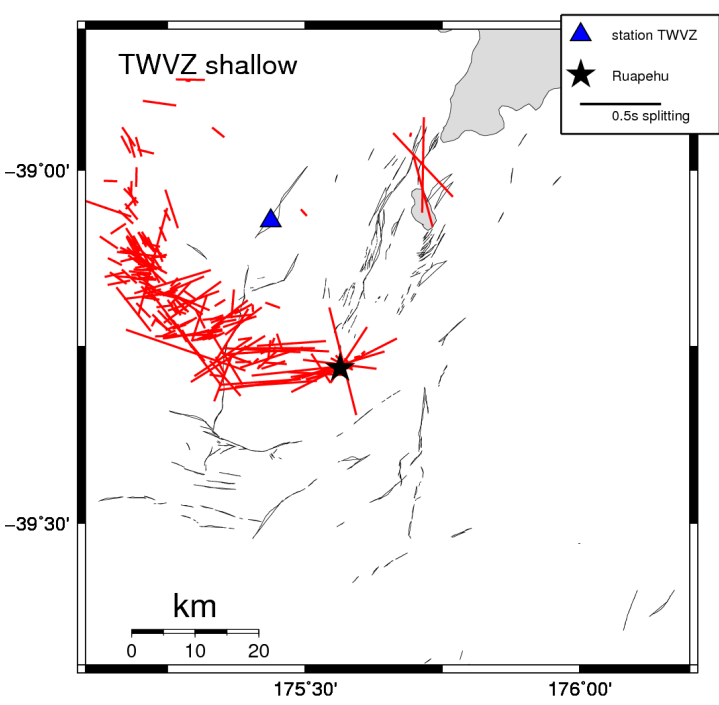

(a) shallow

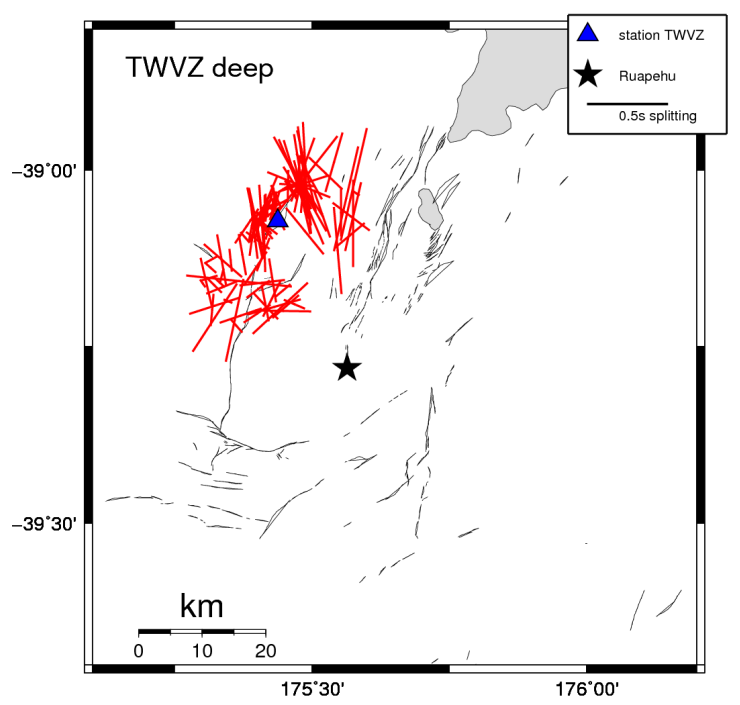

(b) deep

Figure C.17.: Results from station TWVZ 


\section{C.3. Backazimuthal distribution of sources}

The figures below illustrate the backazimuthal distribution of all data that were used for measurements. Yearly maps are shown for 2004-2007. Changes with time can be seen for shallow events at stations NGZ, PKVZ, TUVZ and WTVZ and for deep events at stations FWVZ, NGZ, PKVZ, TWVZ and WTVZ. This possibly indicates some changes in the local seismicity in the vicinity of Mt. Ruapehu but was not further investigated in this study. For most stations where a change in backazimuthal distribution occurred this did not introduce large changes to the shear wave splitting measurements. For example at station NGZ, the backazimuths of deep events differed between 2005 and 2006 (Figures C.18 and C.19), while the resulting mean fast orientation did not change (Table 6.3).
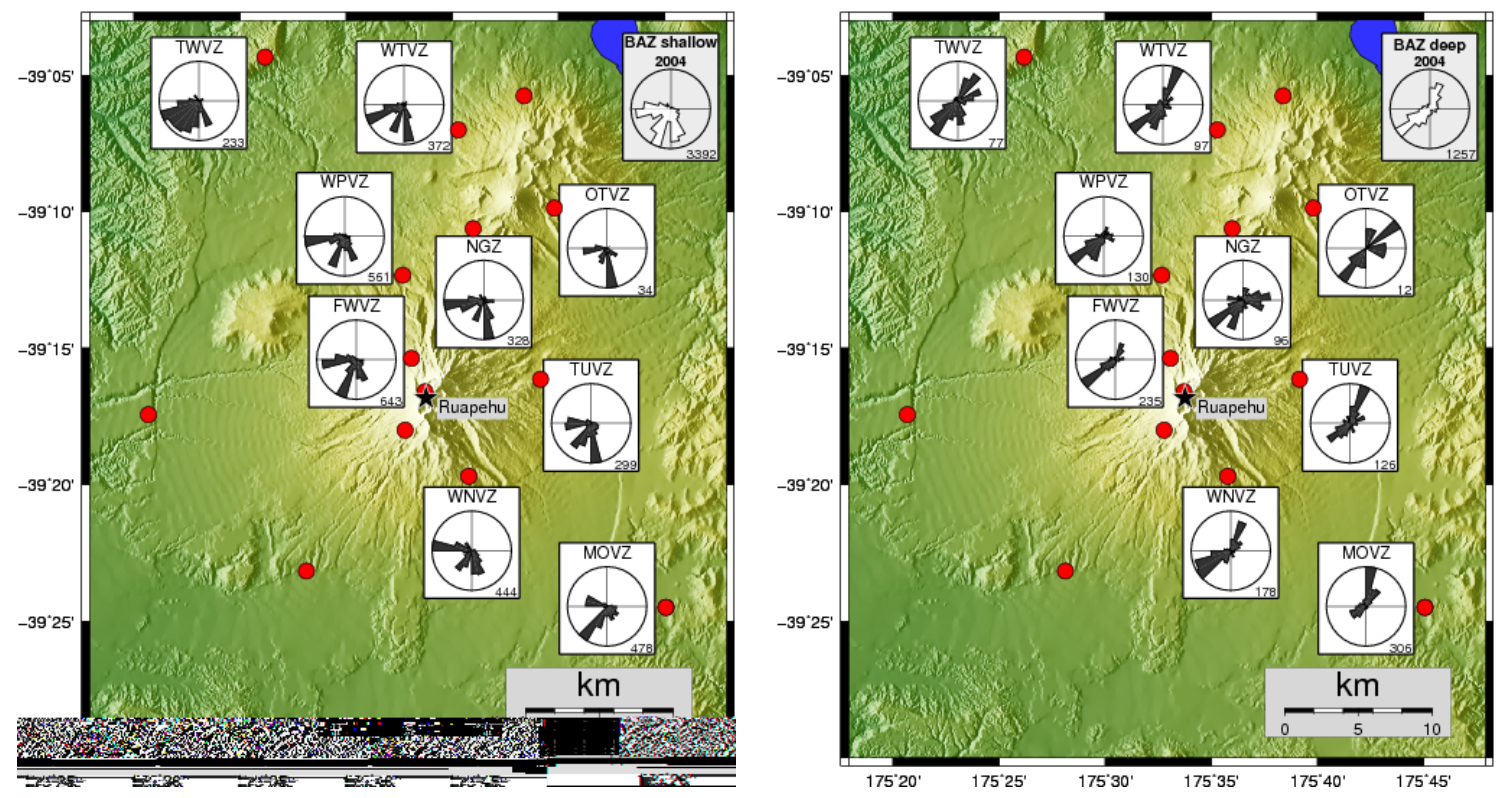

Figure C.18.: Backazimuth of selected event data per station in 2004. Left: Shallow events $(0-30 \mathrm{~km})$. Right: Deep events $(55-300 \mathrm{~km})$. 

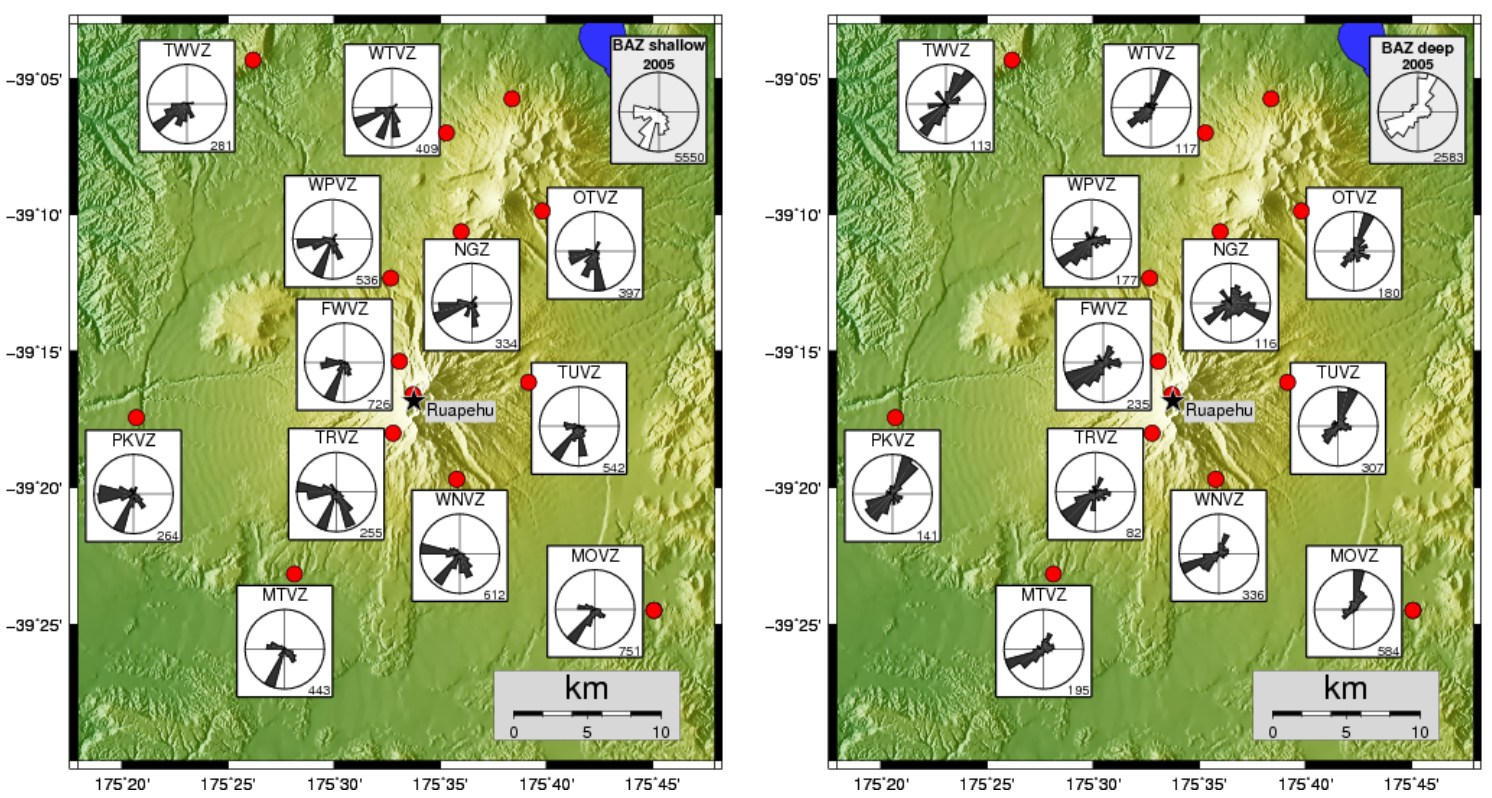

Figure C.19.: Backazimuth of selected event data per station in 2005. Left: Shallow events. Right: Deep events.
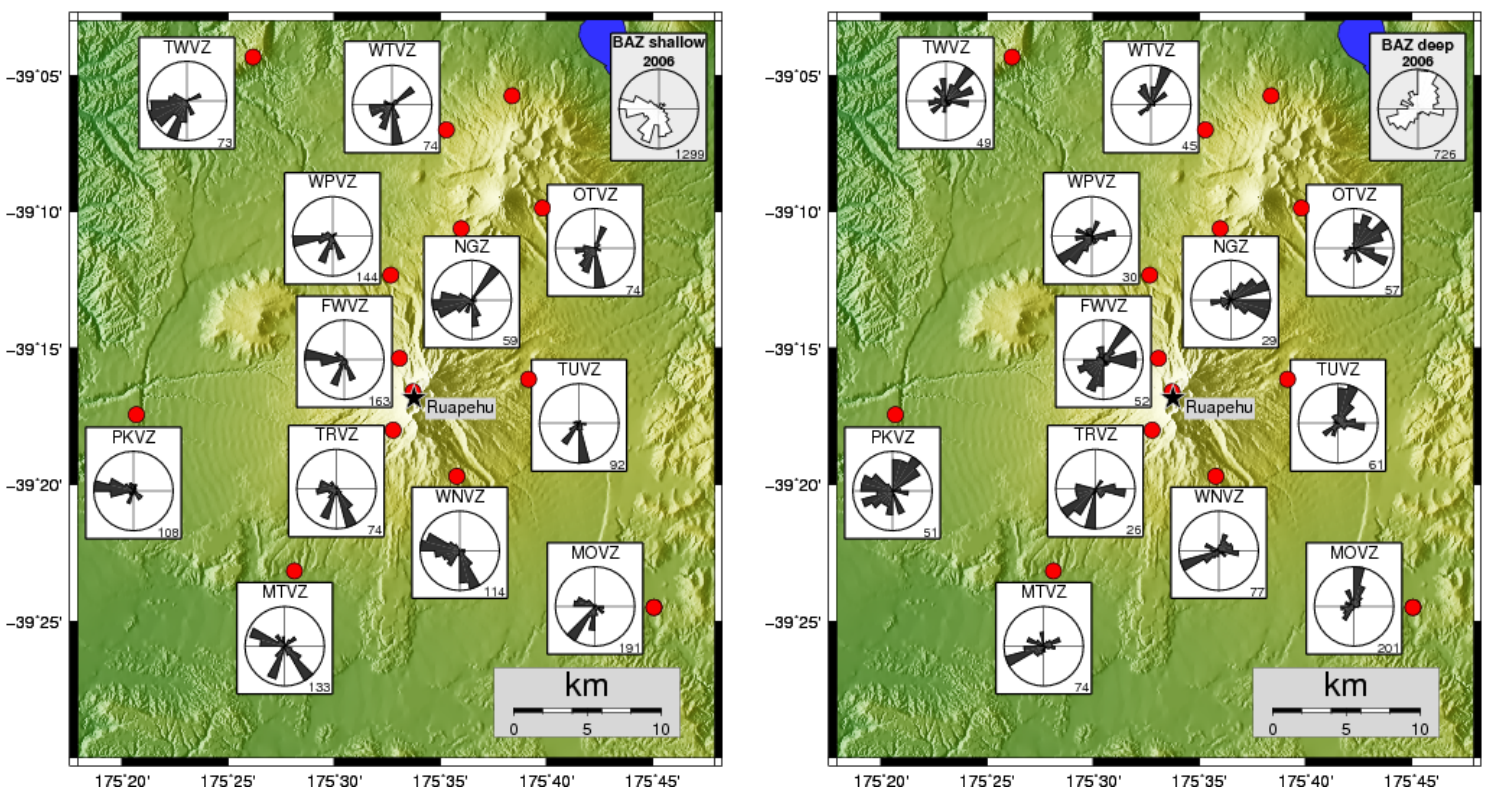

Figure C.20.: Backazimuth of selected event data per station in 2006. Left: Shallow events. Right: Deep events. 

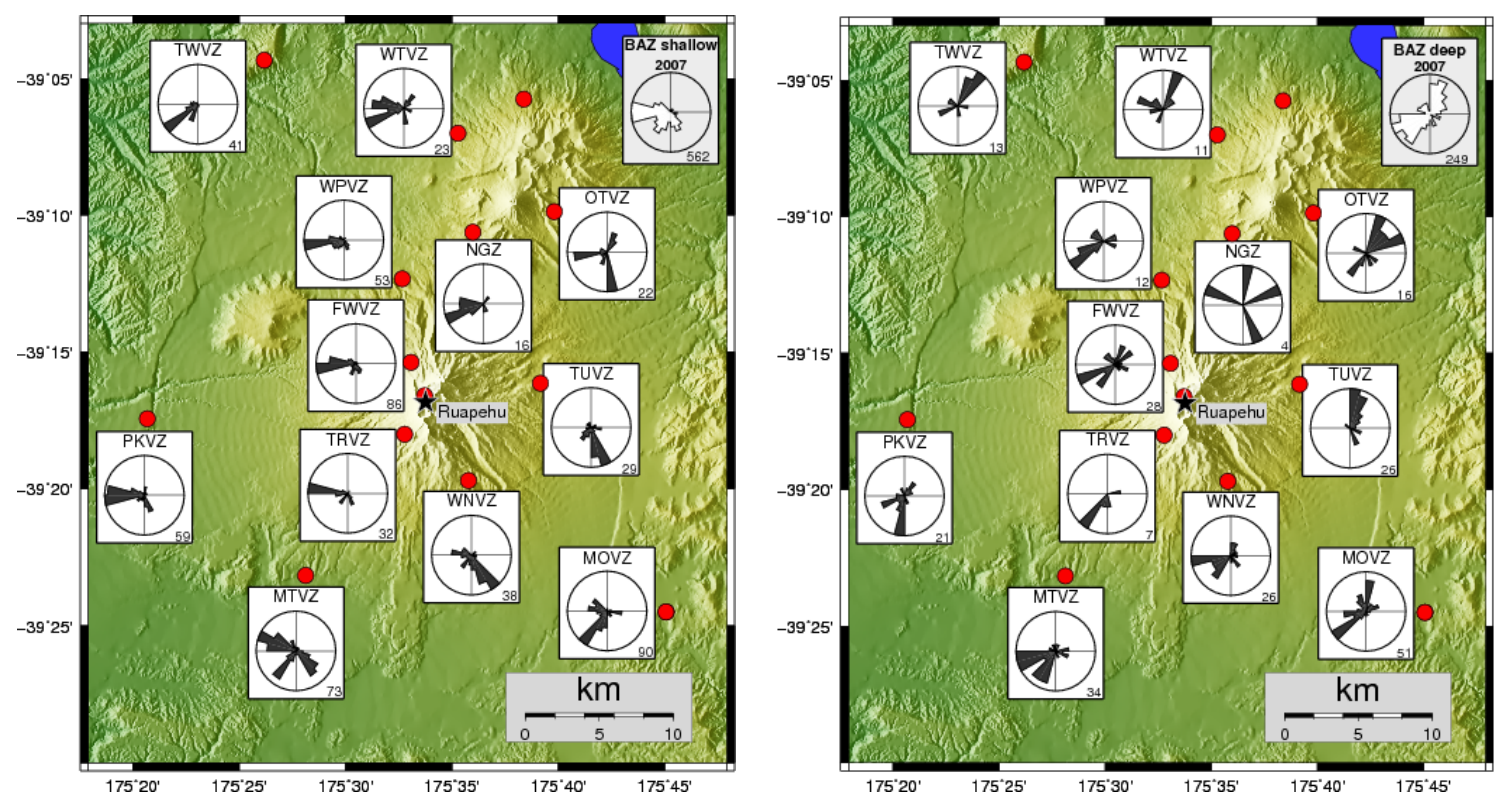

Figure C.21.: Backazimuth of selected event data per station in 2007. Left: Shallow events. Right: Deep events. 


\section{References}

\section{D.L. Anderson.}

Elastic Wave Propagation in Layered Anisotropic Media.

Journal of Geophysical Research, 66:2953, 1961.

E. Angerer, S. Crampin, X.-Y. Li, and T.L. Davis.

Processing, modelling, and predicting time-lapse effects of over-pressured fluidinjection in a fractured reservoir.

Geophysical Journal International, 149(2):267-280, 2002.

P. Armstrong, D. Ireson, E. Gatwick, B. Chmela, E. London, C. Esmersoy, D. Miller, B. Hornby, C. Sayers, M. Schoenberg, et al.

The promise of elastic anisotropy.

International Journal of Rock Mechanics and Mining Sciences and Geomechanics Abstracts, 32(8):377A-378A, 1995.

R.C. Aster, P.M. Shearer, and J. Berger.

Quantitative measurements of shear wave polarizations at the Anza seismic network, southern California: Implications for shear wave splitting and earthquake prediction.

Journal of Geophysical Research, 95:12449-12473, 1990.

R.C. Aster, P.M. Shearer, and J. Berger.

Comments on Quantitative measurements of shear wave polarization at the Anza seismic network, Southern California: Implications for shear wave splitting and earthquake prediction.

Journal of Geophysical Research, 96:6415-6419, 1991.

E. Audoine, M.K. Savage, and K. Gledhill.

Seismic anisotropy from local earthquakes in the transition region from a subduction to a strike-slip plate boundary, New Zealand.

Journal of Geophysical Research, 105(B4):8013-8034, 2000.

E. Audoine, M.K. Savage, and K. Gledhill.

Anisotropic structure under a back arc spreading region, the Taupo Volcanic Zone, New Zealand.

Journal of Geophysical Research, 109:B11305, 2004.

V. Babuška and M. Cara.

Seismic Anisotropy in the Earth.

Modern Approaches in Geophysics. Kluwer Academic Publishers, Boston, 1991. 
V. Babuška, J. Plomerova, and J. Sileny.

Spatial variations of $\mathrm{P}$ residuals and deep structure of the European lithosphere. Geophysical Journal of the Royal Astronomical Society, 79:363-383, 1984.

G.E. Backus.

Long-Wave Elastic Anisotropy Produced by Horizontal Layering. Journal of Geophysical Research, 67:4427, 1962.

R. Bartholin.

Experiments with the Double Refracting Iceland Crystal. 1669.

K.R. Berryman and S. Beanland.

Variation in fault behaviour in different tectonic provinces of New Zealand. Journal of Structural Geology, 13:177-189, 1991.

F. Bianco, M. Castellano, and G. Ventura.

Structural and seismological features of the 1989 Syn-eruptive NNW-SSE fractures system at Mt. Etna.

Geophysical Research Letters, pages 1545-1548, 1998.

F. Bianco, L. Scarfi, E. Del Pezzo, and D. Patane.

Shear wave splitting changes associated with the 2001 volcanic eruption on Mt Etna.

Geophysical Journal International, 167(2):959-967, 2006.

H.M. Bibby, T.G. Caldwell, F.J. Davey, and T.H. Webb.

Geophysical evidence on the structure of the Taupo Volcanic Zone and its hydrothermal circulation.

Journal of Volcanology and Geothermal Research, 68(1-3):29-58, 1995.

F. Birch.

The velocity of compressional waves in rocks to 10 kilobars, Part 1.

Journal of Geophysical Research, 65:1083-1102, 1960.

G.H.R. Bokelmann and H.P. Harjes.

Evidence for temporal variation of seismic velocity within the upper continental crust.

Journal of Geophysical Research, 105(B10):23879-25390, 2000.

D.C. Booth and S. Crampin.

Shear-wave polarizations on a curved wavefront at an isotropic free surface.

Geophysical Journal of the Royal Astronomical Society, 83:31-45, 1985.

M.G. Bostock.

Mantle stratigraphy and evolution of the Slave province.

Journal of Geophysical Research, 103:21, 1998. 
K.B. Broberg.

Cracks and fracture.

Academic Press, London, 1999.

C.J. Bryan and S. Sherburn.

Seismicity associated with the 1995-1996 eruptions of Ruapehu volcano, New Zealand: narrative and insights into physical processes.

Journal of Volcanology and Geothermal Research, 90(1):1-18, 1999.

R. W. Busby, M. Avarez, and R. Wooley.

EarthScope/USArray Transportable Seismic Array spans California. 86(52), 2005.

S. Chevrot, N. Favier, and D. Komatitsch.

Shear wave splitting in three-dimensional anisotropic media.

Geophysical Journal International, 159(2):711-720, 2004.

D.A. Christoffel and W.J. van der Linden.

Macquarie Ridge-New Zealand Alpine Fault transition, volume 19 of Antarctic Research Series.

American Geophysical Union, Washington D.C., 1972.

E.B. Christoffel. Über die Fortpflanzung von Stößen durch elastische feste Körper. Annali di Matematica, 8(1):193-243, 1877.

E.S. Cochran, J.E. Vidale, and Y.G. Li.

Near-fault anisotropy following the Hector Mine earthquake.

Journal of Geophysical Research, 108:2436, 2003.

E.S. Cochran, Y.G. Li, and J.E. Vidale.

Anisotropy in the Shallow Crust Observed around the San Andreas Fault Before and After the 2004 M 6.0 Parkfield Earthquake.

Bulletin of the Seismological Society of America, 96(4B):364-375, 2006.

J.W. Cole.

Structural control and origin of volcanism in the Taupo volcanic zone, New Zealand.

Bulletin of Volcanology, 52(6):445-459, 1990.

S. Crampin.

An introduction to wave propagation in anisotropic media.

Geophysical Journal International, 76:17-28, 1984.

S. Crampin.

Geological and industrial implications of extensive-dilatancy anisotropy.

Nature, 328(6130):491-496, 1987.

S. Crampin.

The fracture criticality of crustal rocks.

Geophysical Journal International, 118:428-438, 1994. 
S. Crampin.

Calculable fluid-rock interactions.

Journal of the Geological Society of London, 156(3):501-514, 1999.

S. Crampin.

Developing stress-monitoring sites using cross-hole seismology to stress-forecast the times and magnitudes of future earthquakes.

Tectonophysics, 338(3):233-245, 2001.

S. Crampin.

Comment on "Local shear wave observations in João Câmara, northeast Brazil" by AF do Nascimento, RG Pearce, and MK Takeya.

Journal of Geophysical Research, 109(B2):B02313, 2004.

S. Crampin and S. Chastin.

A review of shear wave splitting in the crack-critical crust.

Geophysical Journal International, 155:221-240, 2003.

S. Crampin and Y. Gao.

Comment on Systematic Analysis of Shear-Wave Splitting in the Aftershock Zone of the 1999 Chi-Chi, Taiwan, Earthquake: Shallow Crustal Anisotropy and Lack of Precursory Changes, by Yungfeng Liu, Ta-Liang Teng, and Yehuda Ben-Zion. Bulletin of the Seismological Society of America, 95(1):354-360, 2005.

S. Crampin and Y. Gao.

A review of techniques for measuring shear-wave splitting above small earthquakes. Physics of the Earth and Planetary Interiors, 159(1-2):1-14, 2006.

S. Crampin and S. Peacock.

A review of shear-wave splitting in the compliant crack-critical anisotropic Earth. Wave Motion, 41(1):59-77, 2005.

S. Crampin and S.V. Zatsepin.

Modelling the compliance of crustal rock - II. Response to temporal changes before earthquakes.

Geophysical Journal international, 129(3):477-494, 1997.

S. Crampin, R. Evans, B. Uecer, M. Doyle, J.P. Davis, G.V. Yegorkina, and A. Miller. Observations of dilatancy-induced polarization anomalies and earthquake prediction.

Nature, 286:874-877, 1980.

S. Crampin, R. Evans, B.K. Atkinson, et al.

Earthquake prediction: a new physical basis.

Geophysical Journal International, 76:147-156, 1984.

S. Crampin, D.C. Booth, R. Evans, S. Peacock, and J.B. Fletcher.

Changes in shear wave splitting at Anza near the time of the North Palm Springs 
earthquake.

Journal of Geophysical Research, 95(11):197-11, 1990.

S. Crampin, D.C. Booth, R. Evans, S. Peacock, and J.B. Fletcher.

Comment on "Quantitative Measurements of Shear Wave Polarizations at the Anza

Seismic Network, Southern California: Implications for Shear Wave Splitting and Earthquake Prediction" by RC Aster, PM Shearer, and J. Berger.

Journal of Geophysical Research, 96:6403-6414, 1991.

S. Crampin, T. Volti, and R. Stefánsson.

A successfully stress-forecast earthquake.

Geophysical Journal International, 138:F1-F5, 1999.

S. Crampin, T. Volti, and R. Stefánsson.

Response to 'A statistical evaluation of a 'stress-forecast' earthquake' by T. Seher \& I. G. Main.

Geophysical Journal International, 157(1):194-199, 2004.

H.P. Crotwell, T.J. Owens, and J. Ritsema.

The TauP Toolkit: Flexible Seismic Travel-Time and Raypath Utilities.

Seismological Research Letters, 70:154-160, 1999.

D.J. Darby and C.M. Meertens.

Terrestrial and GPS measurements of deformation across the Taupo back arc and Hikurangi forearc regions in New Zealand.

Journal of Geophysical Research, 100(B5):8221-8232, 1995.

J.C. Davis.

Analysis of directional data. In Statistics and Data Analysis in Geology - Second Edition, pages 314-330. John Wiley \& Sons, Inc., 1986.

E. Del Pezzo, F. Bianco, and G. Saccorotti.

Seismic source dynamics at Vesuvius volcano, Italy.

Journal of Volcanology and Geothermal Research, 133(1-4), 2004.

A. F. do Nascimento, F. H. R. Bezerra, and M. K. Takeya.

Ductile Precambrian fabric control of seismic anisotropy in the Açu dam area, northeastern Brazil.

Journal of Geophysical Research, 109:B10311, 2004a.

A.F. do Nascimento, R.G. Pearce, and M.K. Takeya.

Local shear wave observations in João Câmara, northeast Brazil.

Journal of Geophysical Research, 107(B10):2232, 2002.

A.F. do Nascimento, R.G. Pearce, and M.K. Takeya.

Reply to comment by S. Crampin on "Local shear wave observations in João Câmara, northeast Brazil".

Journal of Geophysical Research, 109(B2):B02314, 2004b. 
D. Eberhart-Phillips, M. Reyners, M. Chadwick, and J.M. Chiu.

Crustal heterogeneity and subduction processes: 3-D Vp, Vp/Vs and $\mathrm{Q}$ in the southern North Island, New Zealand.

Geophysical Journal International, 162(1):270-288, 2005.

M. Elkibbi and J.A. Rial.

The Geysers geothermal field: results from shear-wave splitting analysis in a fractured reservoir.

Geophysical Journal International, 162(15):1024-1035, 2005.

M. Elkibbi, M. Yang, and J.A. Rial.

Crack-induced anisotropy models in The Geysers geothermal field.

Geophysical Journal International, 162(3):1036-1048, 2005.

M.S. Evans, J.-M. Kendall, and R.J. Willemann.

Automated SKS splitting and upper-mantle anisotropy beneath Canadian seismic stations.

Geophysical Journal International, 165(3):931-942, 2006.

D.W. Forsyth.

The early structural evolution and anisotropy of the oceanic upper mantle.

Geophysical Journal of the Royal Astronomical Society, 43:103-162, 1975.

D.W. Forsyth and A. Li.

Array analysis of two-dimensional variations in surface wave phase velocity and azimuthal anisotropy in the presence of multipathing interference.

Geophysical Monograph, 157:81-97, 2005.

M.J. Fouch and S. Rondenay.

Seismic anisotropy beneath stable continental interiors.

Physics of the Earth and Planetary Interiors, 158(2-4):292-320, 2006.

J.A. Gamble, R.C. Price, I.E.M. Smith, W.C. McIntosh, and N.W. Dunbar.

${ }^{40} \mathrm{Ar} /{ }^{39} \mathrm{Ar}$ geochronology of magmatic activity, magma flux and hazards at Ruapehu volcano, Taupo Volcanic Zone, New Zealand.

Journal of Volcanology and Geothermal Research, 120(3):271-287, 2003.

Y. Gao, P. Hao, and S. Crampin.

SWAS: A Shear-Wave Analysis System for semi-automatic measurement of shearwave splitting above small earthquakes.

Physics of the Earth and Planetary Interiors, 159(1-2):71-89, 2006.

A. Gerst.

Temporal Changes in Seismic Anisotropy as a New Eruption Forecasting Tool. Master's thesis,

Victoria University of Wellington, New Zealand, 2003. 
A. Gerst and M.K. Savage.

Seismic anisotropy beneath Ruapehu volcano: a possible eruption forecasting tool. Science, 306(5701):1543-1547, 2004.

P. Goldstein and A. Snoke.

SAC availability for the IRIS community.

Incorporated Research Institutions for Seismology Newsletter, 7(1), 2005.

A.E. Gripp and R.G. Gordon.

Young tracks of hotspots and current plate velocities.

Geophysical Journal International, 150(2):321-361, 2002.

A. Gudmundsson.

Emplacement and arrest of sheets and dykes in central volcanoes.

Journal of Volcanology and Geothermal Research, 116(3-4):279-298, 2002.

A. Gudmundsson.

Surface stresses associated with arrested dykes in rift zones.

Bulletin of Volcanology, 65(8):606-619, 2003.

C. Gumiaux, D. Gapais, and J. P. Brun.

Geostatistics applied to best-fit interpolation of orientation data.

Tectonophysics, 376(3-4):241-259, 2003.

A.J. Haines.

A local magnitude scale for New Zealand earthquakes.

Bulletin of the Seismological Society of America, 71(1):275-294, 1981.

T. Hatherton.

Gravity, seismicity, and tectonics of the North Island, New Zealand. New Zealand Journal of Geology and Geophysics, 13:126-144, 1970.

K. Helbig and L. Thomsen.

75-plus years of anisotropy in exploration and reservoir seismics: A historical review of concepts and methods.

Geophysics, 70:9-23, 2005.

H.H. Hess.

Seismic anisotropy of the uppermost mantle under oceans.

Nature, 203(4945):629-631, 1964.

K. Hoernle, J.D.L White, P. van den Bogaard, F. Hauff, D.S. Coombs, R. Werner, C. Timm, D. Garbe-Schönberg, A. Reay, and A.F. Cooper.

Cenozoic intraplate volcanism on New Zealand: Upwelling induced by lithospheric removal.

Earth and Planetary Science Letters, 248(1-2):335-352, 2006. 
N.A. Horspool, M.K. Savage, and S. Bannister.

Implications for intraplate volcanism and back-arc deformation in northwestern New Zealand, from joint inversion of receiver functions and surface waves. Geophysical Journal International, 166(3):1466-1483, 2006.

B.F. Houghton, J.H. Latter, and W.R. Hackett.

Volcanic hazard assessment for Ruapehu composite volcano, taupo volcanic zone, New Zealand.

Bulletin of Volcanology, 49(6):737-751, 1987.

B.F. Houghton, C.J.N. Wilson, M.O. McWilliams, M.A. Lanphere, S.D. Weaver, R.M. Briggs, and M.S. Pringle.

Chronology and dynamics of a large silicic magmatic system; central Taupo volcanic zone, New Zealand.

Geology, 23(1):13-16, 1995.

J.A. Hudson.

Wave speeds and attenuation of elastic waves in material containing cracks. Geophysical Journal of the Royal Astronomical Society, 64(1):133-150, 1981.

A. W. Hurst and P. J. McGinty.

Earthquake swarms to the west of Mt Ruapehu preceding its 1995 eruption. Journal of Volcanology and Geothermal Research, 90(1-2):19-28, 1999.

A.W. Hurst, H.M. Bibby, B.J. Scott, and M.J. McGuinness.

The heat source of Ruapehu crater lake; deductions from the energy and mass balances.

Journal of Volcanology and Geothermal Research, 46(1-2):1-20, 1991.

T. Hurst, B. Scott, C. Werner, and N. Stevens.

Monitoring New Zealand volcanoes.

Tephra: Living with Volcanoes, 21:12-7, 2004.

R. Ikuta and K. Yamaoka.

Temporal variation in the shear wave anisotropy detected using the Accurately Controlled Routinely Operated Signal System (ACROSS).

Journal of Geophysical Research, 109:B09305, 2004.

W.B. Ismal and D. Mainprice.

An olivine fabric database: an overview of upper mantle fabrics and seismic anisotropy.

Tectonophysics, 296(1):145-157, 1998.

D.M. Johnston, B.F. Houghton, V.E. Neall, K.R. Ronan, and D. Paton.

Impacts of the 1945 and 1995-1996 Ruapehu eruptions, New Zealand: An example of increasing societal vulnerability.

Bulletin of the Geological Society of America, 112(5):720-726, 2000. 
S. Kaneshima, M. Ando, and S. Kimura.

Evidence from shear-wave splitting for the restriction of seismic anisotropy to the upper crust.

Nature, 335(6191):627-629, 1988.

S.I. Karato and P. Wu.

Rheology of the Upper Mantle: A Synthesis.

Science, 260(5109):771, 1993.

B.L.N. Kennett, E.R. Engdahl, and R. Buland.

Constraints on seismic velocities in the Earth from traveltimes.

Geophysical Journal International, 122(1):108-124, 1995.

Y.A. Kravtsov and Y.I. Orlov.

Limits of applicability of the method of geometric optics and related problems.

Soviet Physics, Uspekhi, 23(11):750-762, 1980.

T. Kuehler.

Search for temporal changes in seismic attenuation under Mt. Ruapehu volcano, North Island, New Zealand. Master's thesis,

Victoria University of Wellington, New Zealand, 2006.

T. Kuehler, M.K. Savage, and T. Hurst.

Changes in attenuation related to eruptions of Mt. Ruapehu Volcano, New Zealand.

Geophysical Journal International, submitted.

J.H. Latter.

Volcanic earthquakes, and their relationship to eruptions at Ruapehu and Ngauruhoe volcanoes.

Journal of Volcanology and Geothermal Research, 9:293-309, 1981.

S.E. Laubach, J.E. Olson, and J.F.W. Gale.

Are open fractures necessarily aligned with maximum horizontal stress?

Earth and Planetary Science Letters, 222(1):191-195, 2004.

J. Lecointre, K. Hodgson, V. Neall, and S. Cronin.

Lahar-Triggering Mechanisms and Hazard at Ruapehu Volcano, New Zealand.

Natural Hazards, 31(1):85-109, 2004.

V. Levin, D. Okaya, and J. Park.

Shear wave birefringence in wedge-shaped anisotropic regions.

Geophysical Journal International, 168(1):275-286, 2007.

E. Liu, S. Crampin, J. H. Queen, and W. D. Rizer.

Behaviour of shear waves in rocks with two sets of parallel cracks.

Geophysical Journal International, 113(2):509-517, 1993. 
Y. Liu, T.L. Teng, and Y. Ben-Zion.

Systematic Analysis of Shear-Wave Splitting in the Aftershock Zone of the 1999 Chi-Chi, Taiwan, Earthquake: Shallow Crustal Anisotropy and Lack of Precursory Variations.

Bulletin of the Seismological Society of America, 94(6):2330-2347, 2004.

Y. Liu, Y. Ben-Zion, and T.L. Teng.

Reply to "Comment on Systematic Analysis of Shear-Wave Splitting in the Aftershock Zone of the 1999 Chi-Chi, Taiwan, Earthquake: Shallow Crustal Anisotropy and Lack of Precursory Changes, by Yunfeng Liu, Ta-Liang Teng, and Yehuda Ben-Zion," by Stuart Crampin and Yuan Gao.

Bulletin of the Seismological Society of America, 95(1):361-366, 2005.

D. Mainprice, A. Tommasi, H. Couvy, P. Cordier, and D.J. Frost.

Pressure sensitivity of olivine slip systems and seismic anisotropy of Earth's upper mantle.

Nature, 433(7027):731-733, 2005.

L. Margheriti, C. Nostro, A. Amato, and M. Cocco.

Seismic anisotropy: an original tool to understand the geodynamic evolution of the Italian peninsula.

Annali Di Geofisica, XL(3):759-769, 1997.

L. Margheriti, M.F. Ferulano, and M. Di Bona.

Seismic anisotropy and its relation with crust structure and stress field in the Reggio Emilia Region (Northern Italy).

Geophysical Journal International, 167(2):1035-1043, 2006.

K. Marson-Pidgeon, M.K. Savage, K.R. Gledhill, and G. Stuart.

Seismic anisotropy beneath the lower half of the North Island, New Zealand.

Journal of Geophysical Research, 104(B9):20277-20286, 1999.

I. Matcham, M.K. Savage, and K.R. Gledhill.

Distribution of seismic anisotropy in the subduction zone beneath the Wellington region, New Zealand.

Geophysical Journal International, 140(1):1-10, 2000.

W. Menke and V. Levin.

The cross-convolution method for interpreting SKS splitting observations, with application to one and two-layer anisotropic earth models.

Geophysical Journal International, 154(2):379-392, 2003.

V. Miller and M.K. Savage.

Changes in seismic anisotropy after volcanic eruptions: Evidence from Mount Ruapehu.

Science, 293(5538):2231-2233, 2001. 
V. Miller, T. Hurst, and J. Beavan.

Feasibility study for geodetic monitoring of Mt Ruapehu volcano, New Zealand, using GPS.

New Zealand Journal of Geology and Geophysics, 46:41-46, 2003.

H.M. Mooney.

Upper mantle inhomogeneity beneath New Zealand: Seismic evidence.

Journal of Geophysical Research, 75:285-309, 1970.

N. Mortimer.

New Zealand's geological foundations.

Gondwana Research, 7:261-272, 2004.

N. Mortimer, K. Hoernle, F. Hauff, J.M. Palin, W.J. Dunlap, R. Werner, and K. Faure.

New constraints on the age and evolution of the Wishbone Ridge, southwest Pacific Cretaceous microplates, and Zealandia-West Antarctica breakup.

Geology, 34(3):185-188, 2006.

C.G. Munson, C.H. Thurber, Y. Li, and P.G. Okubo.

Crustal shear wave anisotropy in southern Hawaii: Spatial and temporal analysis. Journal of Geophysical Research, 100(B10):20367-20378, 1995.

I.A. Nairn, Tetsuo Kobayashi, and Mitsuhiro Nakagawa.

The 10 ka multiple vent pyroclastic eruption sequence at Tongariro Volcanic Centre, Taupo Volcanic Zone, New Zealand: - Part 1. Eruptive processes during regional extension.

Journal of Volcanology and Geothermal Research, 86(1-4):19-44, 1998.

M. Nakagawa, K. Wada, T. Thordarson, C.P. Wood, and J.A. Gamble.

Petrologic investigations of the 1995 and 1996 eruptions of Ruapehu volcano, New Zealand: formation of discrete and small magma pockets and their intermittent discharge.

Bulletin of Volcanology, 61(1):15-31, 1999.

K. Nakamura.

Volcanoes as possible indicators of tectonic stress orientation - principle and proposal.

Journal of Volcanology and Geothermal Research, 2:1-16, 1977.

K. Nakamura, K.H. Jacob, and J.N. Davies.

Volcanoes as possible indicators of tectonic stress orientation - Aleutians and Alaska.

Pure and Applied Geophysics, 115(1):87-112, 1977.

V.E. Neall, B.F. Houghton, S.J. Cronin, S.L. Donoghue, K.A. Hodgson, D.M. Johnston, J.A. Lecointre, and A.R. Mitchell.

Volcanic hazards at Ruapehu Volcano. 
Volcanic Hazards Information Series, Ministry of Civil Defence, Wellington, 8: 1-30, 1999.

A. Nicolas and N.I. Christensen.

Formation of anisotropy in upper mantle peridotites: A review.

Geodynamics Series, American Geophysical Union, 16:111-123, 1987.

A. Nur and G. Simmons.

Stress-induced velocity anisotropy in rock: an experimental study.

Journal of Geophysical Research, 74:6667-6674, 1969.

O. Nuttli.

The effect of the earth's surface on the S wave particle motion.

Bulletin of the Seismological Society of America, 51(2):237-246, 1961.

S. Özalaybey and M.K. Savage.

Double-layer anisotropy resolved from $\mathrm{S}$ phases.

Geophysical Journal International, 117(3):653-664, 1994.

J. Park and V. Levin.

Seismic anisotropy: Tracing plate dynamics in the mantle.

Science, 296(5567):485-489, 2002.

T. Parsons and G.A. Thompson.

The Role of Magma Overpressure in Suppressing Earthquakes and Topography:

Worldwide Examples.

Science, 253(5026):1399, 1991.

Z. Peng and Y. Ben-Zion.

Systematic analysis of crustal anisotropy along the Karadere-Düzce branch of the North Anatolian fault.

Geophysical Journal International, 159(1):253-274, 2004.

Z. Peng and Y. Ben-Zion.

Spatiotemporal variations of crustal anisotropy from similar events in aftershocks of the 1999 M7. 4 I zmit and M7. 1 Düzce, Turkey, earthquake sequences.

Geophysical Journal International, 160(3):1027, 2005.

D. Piccinini, L. Margheriti, L. Chiaraluce, and M. Cocco.

Space and time variations of crustal anisotropy during the 1997 Umbria-Marche, central Italy, seismic sequence.

Geophysical Journal International, 167(3):1482-1490, 2006.

S.H. Pozgay, D.A. Wiens, J.A. Conder, H. Shiobara, and H. Sugioka.

Complex mantle flow in the Mariana subduction system: Evidence from shear wave splitting.

Geophysical Journal International, 170(1):371-386, 2007. 
R.C. Price, J.A. Gamble, I.E.M. Smith, R.B. Stewart, S. Eggins, and I.C. Wright. An integrated model for the temporal evolution of andesites and rhyolites and crustal development in New Zealand's North Island.

Journal of Volcanology and Geothermal Research, 140(1-3):1-24, 2005.

W. Rabbel and E. Luschen.

Shear wave anisotropy of laminated lower crust at the Urach geothermal anomaly. Tectonophysics, 264(1):219-233, 1996.

\section{Reyners.}

Plate coupling and the hazard of large subduction thrust earthquakes at the Hikurangi subduction zone, New Zealand.

New Zealand Journal of Geology and Geophysics, 41(4):343-354, 1998.

M. Reyners, D. Eberhart-Phillips, G. Stuart, and Y. Nishimura.

Imaging subduction from the trench to $300 \mathrm{~km}$ depth beneath the central North Island, New Zealand, with Vp and Vp-Vs.

Geophysical Journal International, 165(2):565-583, 2006.

R. Robinson.

Temporal variations in coda duration of local earthquakes in the Wellington region, New Zealand.

Pure and Applied Geophysics, 125(4):579-596, 1987.

D.C. Roman, S.C. Moran, J.A. Power, and K.V. Cashman.

Temporal and spatial variation of local stress fields before and after the 1992 eruptions of Crater Peak Vent, Mount Spurr Volcano, Alaska.

Bulletin of the Seismological Society of America, 94(6):2366-2379, 2004.

D.P. Rowlands, R.S. White, and A.J. Haines.

Seismic tomography of the Tongariro Volcanic Centre, New Zealand.

Geophysical Journal International, 163(3):1180-1194, 2005.

Ruapehu Alert Bulletin 01.

Ruapehu volcano: possible Crater Lake changes, alert level remains at level 1.

GNS Science Alert Bulletin, RUA-07/01, 2007.

Ruapehu Alert Bulletin 03.

Ruapehu volcano: Eruption from Ruapehu: Science alert level remains at Level 2. GNS Science Alert Bulletin, RUA-07/03, 2007.

Ruapehu Alert Bulletin 04.

Ruapehu volcano: Alert level remains at level 1; minor eruption now confirmed. GNS Science Alert Bulletin, RUA-06/04, 2006.

A.M. Rubin.

Propagation of Magma-Filled Cracks.

Annual Review of Earth and Planetary Sciences, 23(1):287-336, 1995. 
M.P. Rudzki.

Über die Gestalt elastischer Wellen in Gesteinen, IV: Studie aus der Theorie der Erdbebenwellen.

Bulletin of the Academy of Sciences, Cracow, pages 373-384, 1898.

G. Rümpker and P.G. Silver.

Apparent shear-wave splitting parameters in the presence of vertically varying anisotropy.

Geophysical Journal International, 135(3):790-800, 1998.

G. Russo, G. Giberti, and G. Sartoris.

The influence of regional stresses on mechanical stability of volcanoes: Stromboli (Italy), volume 110 of Geological Society Special Publications.

Volcano Instability on the Earth and Other Planets, 1996.

A. Saiga, Y. Hiramatsu, T. Ooida, and K. Yamaoka.

Spatial variation in the crustal anisotropy and its temporal variation associated with a moderate-sized earthquake in the Tokai region, central Japan.

Geophysical Journal International, 154(3):695-705, 2003.

M. K. Savage, M. Bardout, M. Salmon, S. Greve, and A. Wessel.

Seismic Anisotropy Across a Boundary Between Compression and Extension Above a Subducting Plate, Western North Island, New Zealand. In

Eos, Transactions of the American Geophysical Union, Fall Meet. Suppl., volume 88, 2007.

M.K. Savage.

Lower crustal anisotropy or dipping boundaries? Effects on receiver functions and a case study in New Zealand.

Journal of Geophysical Research, 103(B7)(15):15,069-15,088, 1998.

M.K. Savage.

Seismic anisotropy and mantle deformation: what have we learned from shear wave splitting.

Reviews of Geophysics, 37(1):65-106, 1999.

R. Scarpa and R.I. Tilling, editors.

Monitoring and mitigation of volcano hazards.

Springer-Verlag, Heidelberg, Germany, 1996.

T. Seher and I.G. Main.

A statistical evaluation of a'stress-forecast' earthquake.

Geophysical Journal International, 157(1):187-193, 2004.

S. Sherburn and C.J. Bryan.

The eruption detection system; Mt. Ruapehu, New Zealand.

Seismological Research Letters, 70(5):505-511, 1999. 
S. Sherburn, C.J. Bryan, A.W. Hurst, J.H. Latter, and B.J. Scott. Seismicity of Ruapehu volcano, New Zealand, 1971-1996: a review. Journal of Volcanology and Geothermal Research, 88(4):255-278, 1999.

P.G. Silver.

Seismic Anisotropy Beneath the Continents: Probing the Depths of Geology. Annual Review of Earth and Planetary Sciences, 24:385-432, 1996.

P.G. Silver and W.W. Chan.

Shear wave splitting and subcontinental mantle deformation. Journal of Geophysical Research, 96(B10):16429-16454, 1991.

P.G. Silver and M.K. Savage.

The interpretation of shear-wave splitting parameters in the presence of two anisotropic layers.

Geophysical Journal International, 119(3):949-963, 1994.

C. Small and T. Naumann.

The global distribution of human population and recent volcanism.

Environmental Hazards, 3:93-109, 2001.

E.G.C. Smith, T. Stern, and M. Reyners.

Subduction and back-arc activity at the Hikurangi convergent Margin, New Zealand.

Pure and Applied Geophysics, 129(1):203-231, 1989.

R.T. Smith and B.F. Houghton.

Vent migration and changing eruptive style during the 1800a Taupo eruption: new evidence from the Hatepe and Rotongaio phreatoplinian ashes.

Bulletin of Volcanology, 57(6):432-439, 1995.

W. Snellius.

Eratosthenes Batavus, de terrae ambitus vera quantitate.

Lugduni Batavorum, Colster, 1617.

P. Środa.

Seismic anisotropy of the upper crust in southeastern Poland - effect of the compressional deformation at the EEC margin: Results of CELEBRATION 2000 seismic data inversion.

Geophysical Research Letters, 2006.

S. Stein and M. Wysession.

An Introduction to Seismology, Earthquakes, Earth Structure.

Oxford: Blackwell Publishing, 2003.

T.A. Stern.

A back-arc basin formed within continental lithosphere: the Central Volcanic Region of New Zealand.

Tectonophysics, 112(1-4):385-409, 1985. 


\section{T.A. Stern.}

Asymmetric back-arc spreading, heat flux and structure associated with the Central Volcanic Region of New Zealand.

Earth and Planetary Science Letters, 85(1-3):265-276, 1987.

T.A. Stern, W.R. Stratford, and M.L. Salmon.

Subduction evolution and mantle dynamics at a continental margin: Central North Island, New Zealand.

Reviews of Geophysics, 44(4), 2006.

W.R. Stratford and T.A. Stern.

Crust and upper mantle structure of a continental backarc: central North Island, New Zealand.

Geophysical Journal International, 166(1):469-484, 2006.

R. Sutherland.

Cenozoic bending of New Zealand basement terranes and Alpine Fault displacement: a brief review.

New Zealand Journal of Geology and Geophysics, 42:295-301, 1999.

A. Takada.

The influence of regional stress and magmatic input on styles of monogenetic and polygenetic volcanism.

Journal of Geophysical Research, 99(B7):13-563, 1994.

C. Tang, J.A. Rial, and J.M. Lees.

Shear-wave splitting: A diagnostic tool to monitor fluid pressure in geothermal fields.

Geophysical Research Letters, 32(21):L21317, 2005.

T. Tanimoto and D.L. Anderson.

Lateral heterogeneity and azimuthal anisotropy of the upper mantle-Love and Rayleigh waves 100-250 sec.

Journal of Geophysical Research, 90:1842-1858, 1985.

N.A. Teanby, J.-M. Kendall, R.H. Jones, and O. Barkved.

Stress-induced temporal variations in seismic anisotropy observed in microseismic data.

Geophysical Journal International, 156(3):459-466, 2004a.

N.A. Teanby, J.-M. Kendall, and M. van der Baan.

Automation of shear-wave splitting measurements using cluster analysis.

Bulletin of the Seismological Society of America, 94(2):453-463, 2004b.

L. Thomsen.

Elastic anisotropy due to aligned cracks in porous rock.

Geophysical Prospecting, 43(3):805-829, 1995. 
L. Thomsen.

Understanding Seismic Anisotropy in Exploration and Exploitation.

Society of Exploration Geophysicists, 2002.

J.-C. Thouret, J.A. Lecointre, V.E. Neall, S.J. Cronin, and R.C. Wallace.

The Whangaehu Formation: a massive debris-avalanche from ancestral Ruapehu? In Geological Society of New Zealand, Miscellaneous Publications. 122 A, 2006.

S.R. Tod.

The effects of stress and fluid pressure on the anisotropy of interconnected cracks. Geophysical Journal International, 149(1):149-156, 2002.

S. Toda, R.S. Stein, P.A. Reasenberg, J.H. Dieterich, and A. Yoshida.

Stress transferred by the $1995 \mathrm{M}(\mathrm{w})=6$. 9 Kobe, Japan, shock- Effect on aftershocks and future earthquake probabilities.

Journal of Geophysical Research, 103(B10):24543-24565, 1998.

K. Umakoshi, H. Shimizu, and N. Matsuwo.

Volcano-tectonic seismicity at Unzen Volcano, Japan, 1985-1999.

Journal of Volcanology and Geothermal Research, 112(1):117-131, 2001.

P. Villamor and K.R. Berryman.

Late Quaternary geometry and kinematics of faults at the southern termination of the Taupo Volcanic Zone, New Zealand.

New Zealand Journal of Geology and Geophysics, 49:1-21, 2006a.

P. Villamor and K.R. Berryman.

Evolution of the southern termination of the Taupo Rift, New Zealand.

New Zealand Journal of Geology and Geophysics, 49:23-37, 2006b.

T. Volti and S. Crampin.

A four-year study of shear-wave splitting in Iceland: 1. background and preliminary analysis.

Geological Society of London, Special Publications, 212(1):117-133, 2003a.

T. Volti and S. Crampin.

A four-year study of shear-wave splitting in Iceland: 2. temporal changes before earthquakes and volcanic eruptions.

Geological Society of London, Special Publications, 212:135-149, 2003b.

R.I. Walcott.

Plate motion and shear strain rates in the vicinity of the Southern Alps.

The Royal Society of New Zealand bulletin, 18:5-12, 1979.

K.T. Walker, A.A. Nyblade, S.L. Klemperer, G.H.R. Bokelmann, and T.J. Owens. On the relationship between extension and anisotropy: Constraints from shear wave splitting across the East African Plateau.

Journal of Geophysical Research, 109(B8):B08302, 2004. 
L.M. Wallace, J. Beavan, R. McCaffrey, and D. Darby.

Subduction zone coupling and tectonic block rotations in the North Island, New Zealand.

Journal of Geophysical Research, 109(B12), 2004.

P. Wessel and W.H.F. Smith.

Free software helps map and display data.

Eos, Transactions, American Geophysical Union, 72(441):445-446, 1991.

Ch.K. Wilson, C.H. Jones, P. Molnar, A.F. Sheehan, and O.S. Boyd.

Distributed deformation in the lower crust and upper mantle beneath a continental strike-slip fault zone: Marlborough fault system, South Island, New Zealand. Geology, 32(10):837-840, 2004.

C.J.N. Wilson, B.F. Houghton, M.O. McWilliams, M.A. Lanphere, S.D. Weaver, and R.M. Briggs.

Volcanic and structural evolution of Taupo Volcanic Zone, New Zealand: a review. Journal of Volcanology and Geothermal Research, 68(1-3):1-28, 1995.

C.S. Witham.

Volcanic disasters and incidents: A new database.

Journal of Volcanology and Geothermal Research, 148(3-4):191-233, 2005.

R. Wood and D. Barker.

Tectonic evolution of the Resolution Ridge System, New Zealand: insights gained through UNCLOS surveying for natural prolongation. In

Eos, Transactions, American Geophysical Union, volume 86(52) of Fall Meeting Supplements, Abstract \# T12C-07, 2005.

J. Wu, S. Crampin, Y. Gao, P. Hao, T. Volti, and Y.T. Chen.

Smaller source earthquakes and improved measuring techniques allow the largest earthquakes in Iceland to be stress forecast (with hindsight).

Geophysical Journal International, 166(3):1293-1298, 2006.

A. Wüstefeld and G. Bokelmann.

Null Detection in Shear-Wave Splitting Measurements.

Bulletin of the Seismological Society of America, 97(4):1204-1211, 2007.

M. Yang, M. Elkibbi, and J.A. Rial.

An inversion scheme to model subsurface fracture systems using shear wave splitting polarization and delay time observations simultaneously.

Geophysical Journal International, 160(3):939-947, 2005.

S.V. Zatsepin and S. Crampin.

Modelling the compliance of crustal rock - I. Response of shear-wave splitting to differential stress.

Geophysical Journal International, 129(3):477-494, 1997. 
J.C. Zinke and M.D. Zoback.

Structure-related and stress-induced shear-wave velocity anisotropy: Observations from microearthquakes near the Calaveras Fault in Central California.

Bulletin of the Seismological Society of America, 90(5):1305-1312, 2000.

M.D. Zoback and J. Townend.

Implications of hydrostatic pore pressures and high crustal strength for the deformation of intraplate lithosphere.

Tectonophysics, 336(1):19-30, 2001. 


\section{Index}

Anderson (1961), 54

Angerer et al. (2002), 49, 58, 60

Armstrong et al. (1995), 22, 24

Aster et al. (1990), 58

Aster et al. (1991), 58

Audoine et al. (2000), 63, 64

Audoine et al. (2004), 63, 64, 76

Babuška and Cara (1991), 22, 25, 27, $28,37,42,43$

Babuška et al. (1984), 54

Backus (1962), 23, 24, 43

Bartholin (1669), 37

Berryman and Beanland (1991), 8

Bianco et al. (1998), 63

Bianco et al. (2006), 46, 61, 62

Bibby et al. (1995), 8

Birch (1960), 27

Bokelmann and Harjes (2000), 30, 58, 60

Booth and Crampin (1985), 47

Bostock (1998), 54

Broberg (1999), 28

Bryan and Sherburn (1999), 13, 68

Busby et al. (2005), 54

Chevrot et al. (2004), 46, 47

Christoffel and van der Linden (1972), 6

Christoffel (1877), 40

Cochran et al. (2003), 28, 31

Cochran et al. (2006), 58, 76

Cole (1990), 8, 9, 63

Crampin and Chastin (2003), 34, 49, 57,59

Crampin and Gao (2005), 59

Crampin and Gao (2006), 46, 49, 55, $64,82,99$

Crampin and Peacock (2005), 29, 142
Crampin and Zatsepin (1997), 29, 30

Crampin et al. (1980), 32

Crampin et al. (1984), 29, 54

Crampin et al. (1990), 58

Crampin et al. (1991), 58

Crampin et al. (1999), 59

Crampin et al. (2004), 59

Crampin (1984), 39

Crampin (1987), 29

Crampin (1994), 23, 28, 31, 33, 57

Crampin (1999), 28, 63

Crampin (2001), 60

Crampin (2004), 32

Crotwell et al. (1999), 71

Darby and Meertens (1995), 63

Davis (1986), 84

Del Pezzo et al. (2004), 59

Eberhart-Phillips et al. (2005), 7, 137

Elkibbi and Rial (2005), 58, 90

Elkibbi et al. (2005), 30, 46, 57

Evans et al. (2006), 55, 87, 129

Forsyth and Li (2005), 54

Forsyth (1975), 54

Fouch and Rondenay (2006), 22, 24, 46, $48,53,54,57$

Gamble et al. (2003), 11-13, 16

Gao et al. (2006), 90, 141

Gerst and Savage (2004), 1, 17, 45, 47, 49, 55, 61, 63, 64, 66, 67, 87, 92, 98-100, 102, 104, 127

Gerst (2003), 9, 24, 35, 36, 47, 64, 68, 70, 71, 84, 87, 92, 99, 101, 128, 129, 134, 162

Goldstein and Snoke (2005), 71, 143, 157

Gripp and Gordon (2002), 7

Gudmundsson (2002), 34 
Gudmundsson (2003), 34

Gumiaux et al. (2003), 84

Haines (1981), 92

Hatherton (1970), 10

Helbig and Thomsen (2005), 24

Hess (1964), 25, 26, 54

Hoernle et al. (2006), 5

Horspool et al. (2006), 28

Houghton et al. (1987), 11, 13, 14, 18

Houghton et al. (1995), 9

Hudson (1981), 31

Hurst and McGinty (1999), 68

Hurst et al. (1991), 15

Hurst et al. (2004), 3, 15, 17, 18

Ikuta and Yamaoka (2004), 60

Ismal and Mainprice (1998), 25

Johnston et al. (2000), 13-15

Kaneshima et al. (1988), 30, 31

Karato and $\mathrm{Wu}$ (1993), 26

Kennett et al. (1995), 71

Kravtsov and Orlov (1980), 46

Kuehler et al. (submitted), 31

Kuehler (2006), 64

Latter (1981), 16, 36

Laubach et al. (2004), 30

Lecointre et al. (2004), 12

Levin et al. (2007), 48, 52, 87

Liu et al. (1993), 30

Liu et al. (2004), 59

Liu et al. (2005), 59

Mainprice et al. (2005), 25, 26

Margheriti et al. (1997), 57

Margheriti et al. (2006), 57

Marson-Pidgeon et al. (1999), 64

Matcham et al. (2000), 85

Menke and Levin (2003), 48, 53

Miller and Savage (2001), 1, 61, 63-65, 98

Miller et al. (2003), 17, 18

Mooney (1970), 7

Mortimer et al. (2006), 5

Mortimer (2004), 5

Munson et al. (1995), 58

Nairn et al. (1998), 63

Nakagawa et al. (1999), 13, 18, 35
Nakamura et al. (1977), 34

Nakamura (1977), 10, 34

Neall et al. (1999), 15

Nicolas and Christensen (1987), 25, 26

Nur and Simmons (1969), 30

Nuttli (1961), 47, 55

Park and Levin (2002), 56

Parsons and Thompson (1991), 34

Peng and Ben-Zion (2004), 28, 55, 76, $84,88,129$

Peng and Ben-Zion (2005), 58, 59

Piccinini et al. (2006), 61

Pozgay et al. (2007), 56, 76

Price et al. (2005), 10, 13

Rabbel and Luschen (1996), 58

Reyners et al. (2006), 7, 8, 12, 138

Reyners (1998), 6

Robinson (1987), 92

Roman et al. (2004), 35

Rowlands et al. (2005), 11, 12

Ruapehu Alert Bulletin 01 (2007), 16

Ruapehu Alert Bulletin 03 (2007), 14, 16

Ruapehu Alert Bulletin 04 (2006), 14

Rubin (1995), 34

Rudzki (1898), 24

Russo et al. (1996), 34

Saiga et al. (2003), 59

Savage et al. (2007), 98

Savage (1998), 28

Savage (1999), 22, 45, 46, 48, 53, 56, 87,143

Scarpa and Tilling (1996), 3, 17

Seher and Main (2004), 59

Sherburn and Bryan (1999), 18

Sherburn et al. (1999), 13, 16, 18, 19

Silver and Chan (1991), 22, 26, 46, 48, $52,57,75-77,79,139,143$

Silver and Savage (1994), 45, 48

Silver (1996), 26, 56

Small and Naumann (2001), 3

Smith and Houghton (1995), 8

Smith et al. (1989), 8

Snellius (1617), 47

Stein and Wysession (2003), 24, 37 
Stern et al. (2006), 5, 6

Stern (1985), 8

Stern (1987), 8-10

Stratford and Stern (2006), 8

Sutherland (1999), 6

Takada (1994), 36

Tang et al. (2005), 58, 60

Tanimoto and Anderson (1985), 54

Teanby et al. (2004a), 60, 82, 128, 129, 139

Teanby et al. (2004b), 75, 76, 79, 81$83,87,143,151,152,157$

Thomsen (1995), 31

Thomsen (2002), 22, 24, 37, 45

Thouret et al. (2006), 12, 15

Toda et al. (1998), 36

Tod (2002), 30

Umakoshi et al. (2001), 34

Villamor and Berryman (2006a), 7, 63

Villamor and Berryman (2006b), 8, 10

Volti and Crampin (2003a), 59

Volti and Crampin (2003b), 59, 61, 63

Walcott (1979), 7, 63

Walker et al. (2004), 26

Wallace et al. (2004), 6, 7, 9, 10, 63

Wessel and Smith (1991), 143, 157

Wilson et al. (1995), 8-10

Wilson et al. (2004), 28

Witham (2005), 2, 3

Wood and Barker (2005), 5

Wu et al. (2006), 59

Yang et al. (2005), 58

Zatsepin and Crampin (1997), 33

Zinke and Zoback (2000), 28

Zoback and Townend (2001), 36

Özalaybey and Savage (1994), 48

Środa (2006), 57

do Nascimento et al. (2002), 32

do Nascimento et al. (2004a), 32

do Nascimento et al. (2004b), 32

Rümpker and Silver (1998), 76

Wüstefeld and Bokelmann (2007), 52 